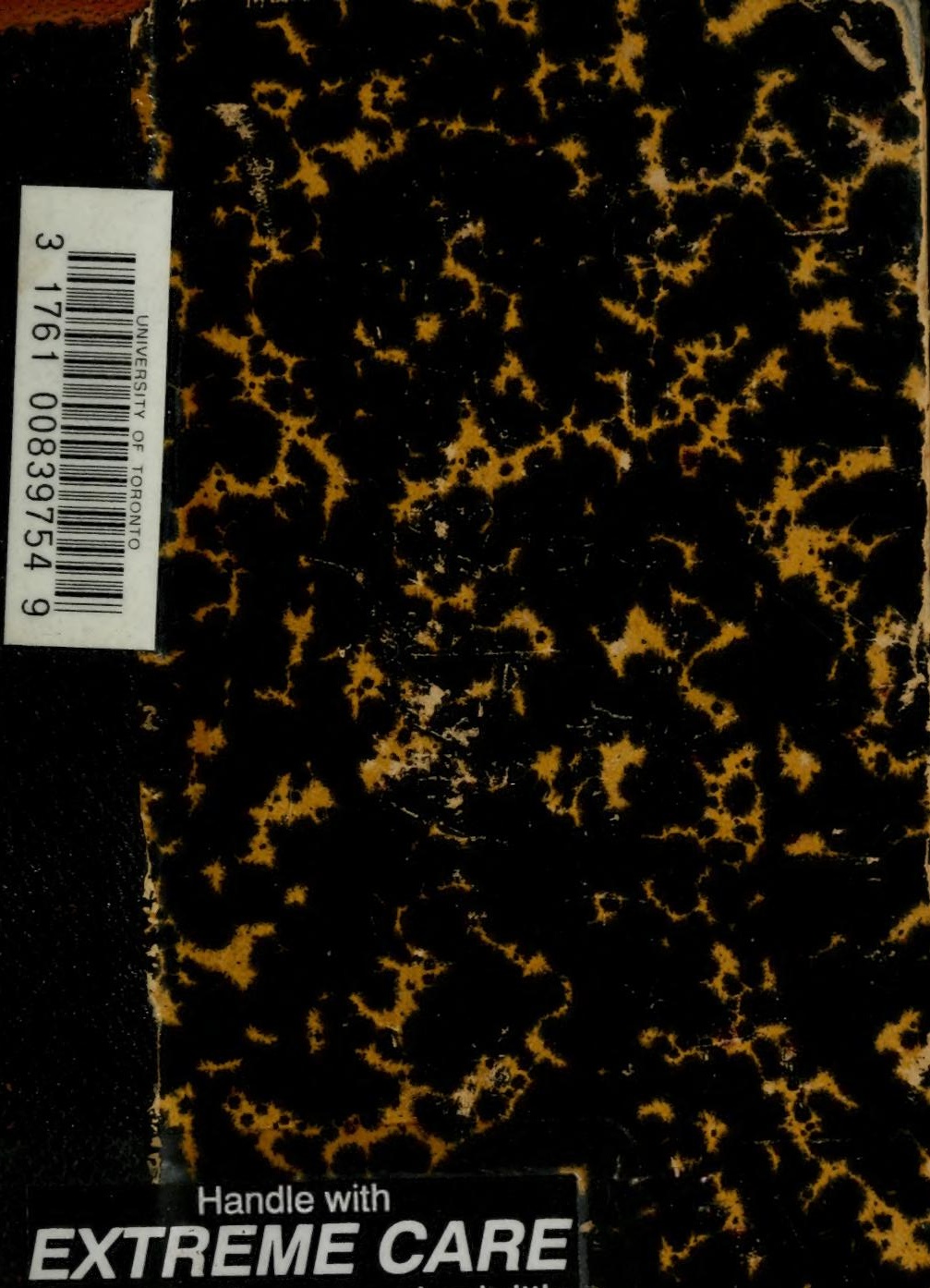

This volume is damaged or brittle and CANNOT be repaired!

- photocopy only if necessary

- return to staff

- do not put in bookdrop

Gerstein Science Information Centre 


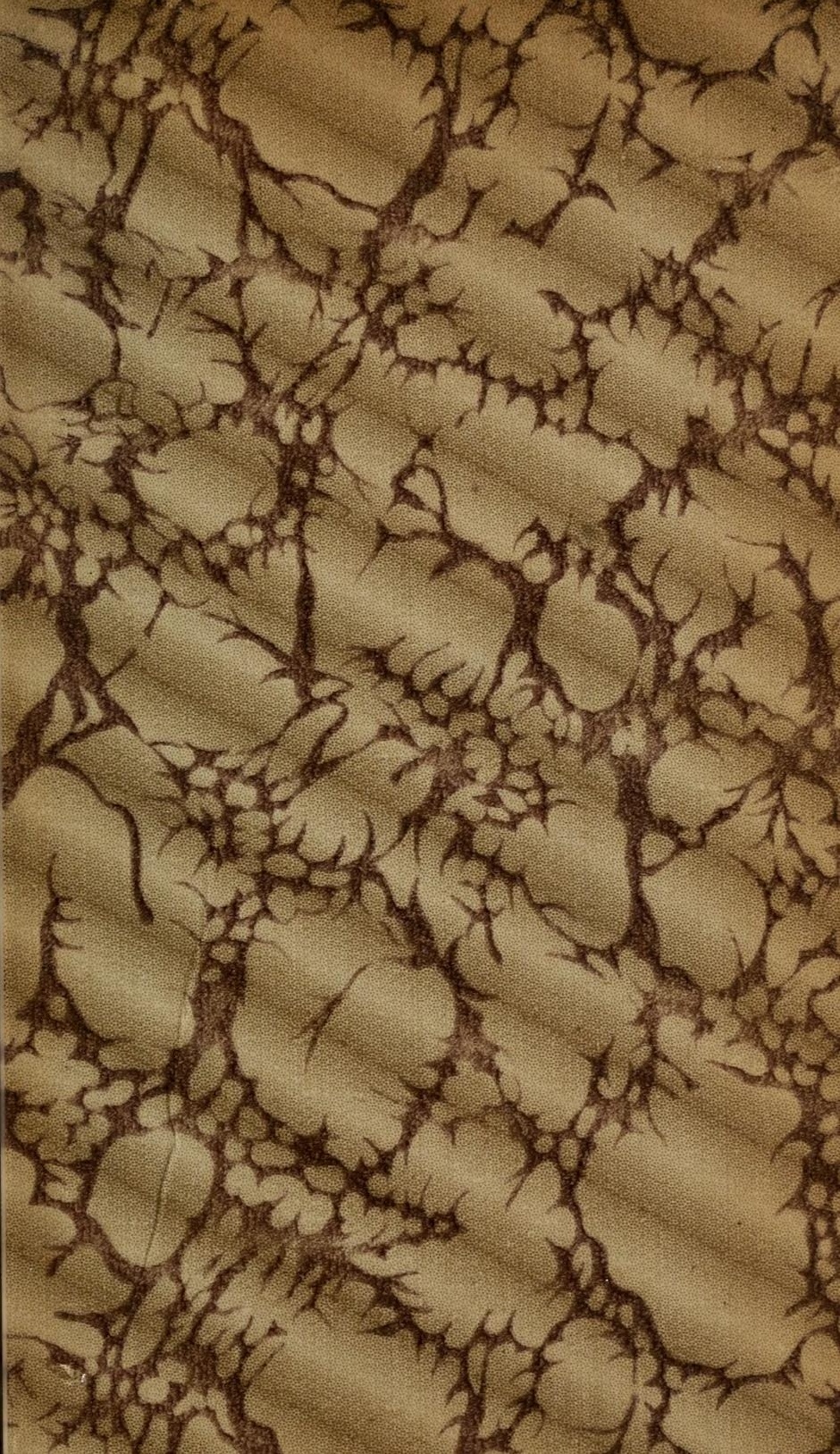







\section{$40 \%$ \\ $5 / 2 / 24$}

\section{Les Problèmes de la Sexualité}



Bibliothèque de Philosophie scientifique

\section{MAURICE CAULLERY}

PROFESSEUR A L'UNIVERSITÉ DE PARIS

Les

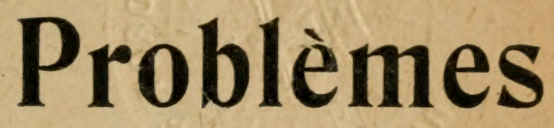

de la

Sexualité

AVEC 48 FIGURES DANS LE TEXTE

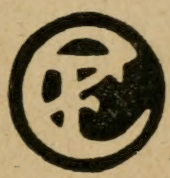

$$
\frac{200966}{\left.213\right|^{26}}
$$

\section{PARIS}

ERNEST FLAMMARION, ÉDITEUR

26 , RUE RACINR, 26

1919

Tous droits de traduction, d'adaptation et de reproduction réservés pour tous les pays. 


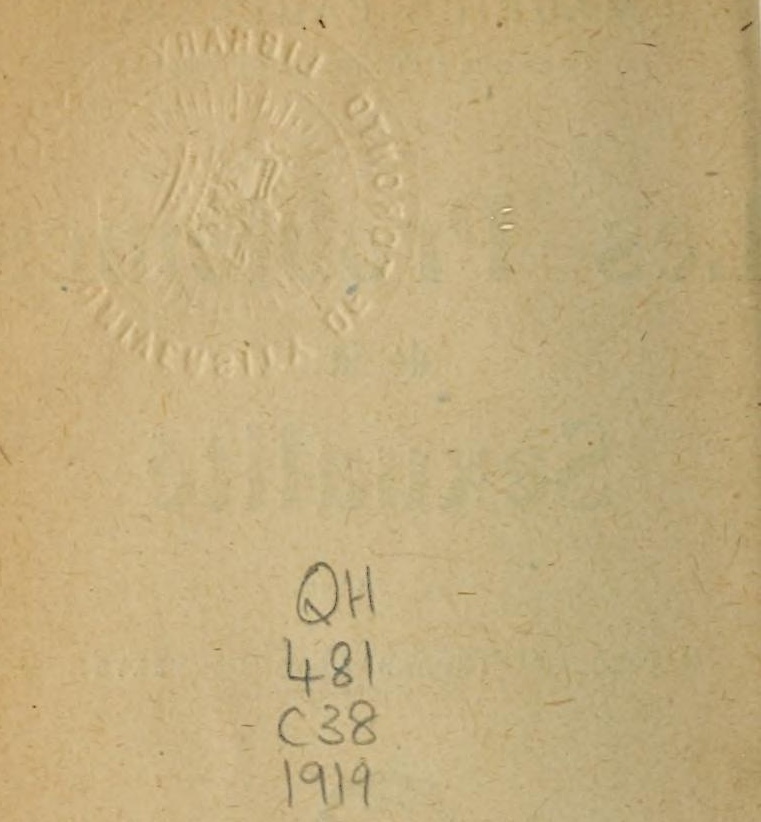




\section{AVANT-PROPOS}

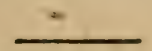

S'il est une notion familière, c'est bien celle du sexe, chez les animaux supérieurs tout au moins. Point n'est besoin davantage d'insister sur la place considérable que tient, dans le domaine du conscient ou de l'inconscient, la Sexualité.

Ce n'est donc une question indifférente pour personne, en dehors même de toute préoccupation scientifique pure.

Mais, à ce dernier point de vue également, la Sexualité est un des ehapitres les plus attirants de la biologie contemporaine, l'un de ceux que l'on a le plus travaillés et enrichis au cours des dernières années. Examinée, non plus seulement chez l'homme et les animaux qui s'en rapprochent, mais dans l'ensemble des êtres vivants, animaux, végétaux et proI stes - ces derniers qu'on ne sait pas toujours $r$ ttacher nettement à un règne ou à l'autre, - la Se xualité apparait d'abord avec une diversité extrême, $\mathrm{m}$ ais sous laquelle on a déjà réussi à reconnaître uı e merveilleuse unité des phénomènes fondamentaux : la synthèse est déjà largement associée à l'analyse des faits.

J'ai donc cru devoir répondre à l'invitation de M. Gustare Le Bon. Ayant lu dans la Revue scienti- 
fique, l'an dernier, la leçon d'ouverture du cours que j'ai professé, en 1911-1912, à la Sorbonne, sur ce sujet, il m'arait proposé d'en tirer un lirre pour la Bibliothèque de Philosophie scientifique.

J'espère que le public accueillera avec intérèt un effort destiné à mettre à la portée des lecteurs cultivés les résultats les plus généraux des recherches sur les problèmes du sexe dans l'ensemble des organismes.

La logique eût voulu peut-être qu'en accord arec les idées transformistes qui dominent la biologie contemporaine, je partisse de l'étude des êtres inférieurs: j'aurais cherché en eux les ébauches les plus simples, les premiers rudiments de la Sexualité, et j'en aurais suivi la différenciation et les étapes successives dans la double série animale et végétale. En d'autres termes, j'en aurais retracé la phylogénie.

Je ne l'ai cependant pas fait. Si c'est là l'ordre le plus logique, il est aussi extrêmement difficile à reconstituer d'une façon authentique. De plus, il exigerait du lecteur, dès le début du livre, des connaissances étendues et précises sur les organismes inférieurs, et il conduirait à un exposé trop technique, qui ne conviendrait peut-être qu'à des biologistes de profession.

J'ai donc préféré partir des notions les plus familières. Et si les origines de la Sexualité sont, au point de vue purement philosophique, l'un des aspects les plus importants du problème, son allure chez les animaux supérieurs est bien, par contre, ce qui intéresse le plus immédiatement et le plus légitimement la généralité des lecteurs. J'ai accordé, pour cette raison, à des êtres plus proches de nous, la place principale dans ce volume. On trouvera, à la fin, un exposé succinct de la question en ce qui concerne les animaux inférieurs et les végétaux. Cela suffira, du 
reste, à juger de l'unité générale des phẻnomènes. Je me suis efforcé de définir, au fur et à mesur? toutes les notions et tous les tęrmes que j'introduisais. J'espère done que l'on pourra aisément suivre l'exposé. Les divisions principales renseigneront aussitôt sur la disposition des parties qui pourraient intéresser plus spécialement chaque lecteur.

La plupart des conceptions scientifiques actuelles sur la Sexualité et les faits précis sur lesquels elles reposent sont d'acquisition récente. Je n'ai pas cherché à présenter l'édifice comme achevé, ni les divers problèmes comme parfaitement clairs et définivement résolus. Je n'ai dissimulé ni les obscurités ni les lacunes qu'une légion d'ardents ouvriers de science y décourre chaque jour et s'applique à faire disparaître.

Dans l'effort général vers la synthèse, on est trop souvent porté à méconnaître le caractère provisoire de beaucoup de notions initiales. C'est précisément une des faiblesses, dans les tendances dominantes, de la Biologie contemporaine. Des hypothèses ont servi de ciment entre les blocs des fondations de l'édifice. A mesure qu'il montait, c'est à de nouvelles hypothèses qu'on recourait pour l'assemblage des matériaux, sans toujours se rendre compte que leur valeur était entièrement subordonnée à celle des premières faites. Ou plutôt, on oublie que celles-ci sont des hypothèses et on leur confère indûment le caractère de certitudes. Telle a été, chez de trop nombreux esprits, la fortune regrettable des théories de A. Weismann sur l'Hérédité. Elles imprègnent, directement ou indirectement, la mentalité contemporaine. Comme beaucoup d'autres, les problèmes de la sexualité ont évolué récemment, sous l'influence et dans le cadre de ces idées weismanniennes. Je n'ai pu faire abstraction de celles-ci, sous peine de rendre méconnais- 
sables bien des questions actuelles. Mais je tiens à faire entendre, dès le seuil de ce livre, une note de scepticisme à l'égard de beaucoup de pseudo-dogmes. On la retrouvera, dans les diverses parties, en des points ou beaucoup d'esprits ne la perçoivent plus.

La philosophie scientifique consiste peut-être avant tout à s'abstenir de tout dogmatisme, à prendre nettement conscience de la relativité de nos connaissances et à entrevoir, à travers les ruines de celles de la veille et les fissures de celles du présent, les constructions de l'avenir.

Paris, avril 1913. 


\section{INTRODUCTION}

\section{LES CELLULES SEXUELLES OU " GAMĖTES *}

Omne vivum ex ovo, a dit Harvey, dès le $\mathrm{xv}_{1}{ }^{e}$ siècle. La théorie cellulaire, œuvre du xix ${ }^{\mathrm{e}}$ siècle, a conféré à cet aphorisme une précision supérieure. Tout être vivant est issu d'un œuf et cet œuf est toujours une cellule, cellule unique et typique. Ainsi les organismes les plus compliqués en organisation, associations de myriades de cellules aux différenciations multiples, l'homme comme tous les autres, passent d'abord par un état unicellulaire, où ils sont comparables au plus humble des Protozoaires.

La cellule œuf a cependant un caractère particulier en quoi se résume la Sexualité. Elle est, comme on dit aujourd'hui, un zygote, c'est-à-dire le résultat de la fusion intime de deux cellules préexistantes. Et ces deux cellules proviennent en général de deux individus distincts; enfin elles sont différenciées l'une par rapport à l'autre. Cesont les gamètes: l'ovule ou gamète femelle, le spermatozoïde ou gamète mâle. Leur fusion est le phénomène de la fécondation. Le zygote, produit de la fécondation, est l'œuf.

Toute la série des processus morphologiques ou physiologiques qui constitue la genèse des gamètes 
et qui aboutit à la réalisation de l'œuf, peut ainsi ètre considérée, en quelque sorte, comme le centre et le jivot des phénomènes sexucls. Il est donc indiqué de chercher tout d'abord à connaitre l'histoire des gamètes, de retracer, dans leurs traits essentiels, leur structure, leur genèse, leur rencontre. Ce sera l'objet des premiers chapitres de cet ourrage, la base nécessaire et l'introduction naturelle à l'étude des problèmes de la Sexualité. 


\section{CHAPITRE 1}

\section{LES GAMETES MÛRS}

\$1. - L'ovule, ou gamète femelle. - L'ovule mûr. ou gamète femelle, est une cellule volumineuse par rapport aux autres éléments de l'organisme, mais de dimensions très variées suivant les animaux. L'ovule humain, petit comme celui de tous les Nammifères ${ }^{1}$, est une -sphère de $0^{\text {mm }}, 13$ de diamètre, à peu près invisible à l'œil nu, tandis que celui de la poule, le jaune de l'œuf, mesure environ $20^{\mathrm{mm}}$ de diamètre. Ces deux nombres donnent une idée des rariations qu'offrent les dimensions de l'élément femelle. La forme sphérique est la plus répandue, mais elle n'est pas la seule. Par exemple, l'ovule

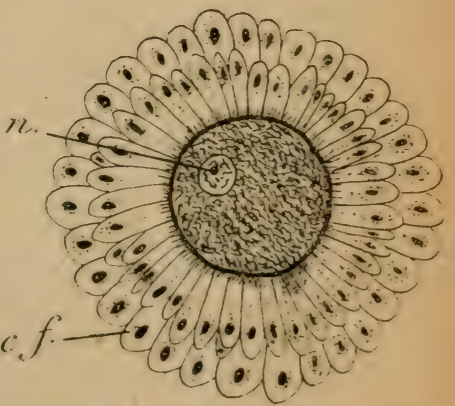

FIG. 1. - Orule de Souris encore dans l'ovaire et envoioppé de celluies folliculaires $c f ; n$, noyau de lovule (daphes Sobotta).

\section{des hydres d'eaudouce}

a conservé la propriété, qu'ont les cellules primitives, de se déformer à la façon des amibes ou des glo-

1. Sauf ceux des Monotrèmes (Ornithorhynque, Echidné). 
bules blancs du sang, en émettant des prolongements temporaires (pseudopodes) en tous sens. Chez beaucoup d'Insectes, tels que les mouches, l'ovule est allongé et en forme d'ellipsoïde (cf. fig. 3).

L'ovule est parfois une cellule nue, comme c'est le cas chez les Hydres, les Oursins, les Etoiles de mer, etc.; mais le plus souvent il est recouvert d'une membrane, soit mince, soit épaisse, lisse ou ornementée. Dans le der-

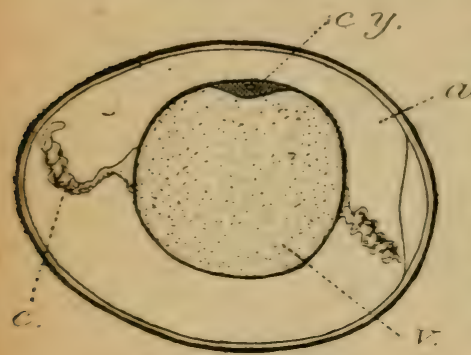

Fic. 2. - CEuf de poule: $C_{y}$, cyiopiasme (formant un disque su fiờe supérieur du jaune); $v$, vitellus (le jaune); $a$, albumine (le blanc); $c$, chalaze. nier cas, la membrane présente généralement un orifice, ou micropyle, par où pénètre le spermatozoïde fécondateur. En outre l'ovule peut ètre enveloppé par des réserves nutritives supplémentaires, enfermées ellesmêmes dans une coque résistante. L'œuf des Oiseaux (fig. 2) en est un exemple typique et connu de tout le monde; l'ovule proprement dit est le jaune; le blanc est une réserve d'albumine supplémentaire.

La caractéristique essentielle de l'ovule, d'où résulte son volume, est l'élaboration par lui et l'accumulation dans sa substance, ou cytoplasme, de matières de réserves - albuminoïdes, lécithines, graisses, etc. qu'on désigne du nom collectif de vitellus. C'est à leurs dépens que l'embryon édifiera ses tissus, jusqu'au moment où il possédera lui-même des organes lui permettant de puiser directement sa nourriture au

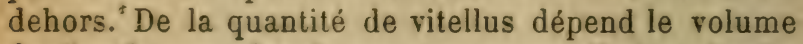
de l'œuf; son abondance est en corrélation étroite 
arec les conditions du développement de l'embryon et influe d'une façon capitale sur la forme de ce développement. Le vitellus, en effet, n'est pas de la substance vivante, mais une matière inerte déposée au sein de celle-ci. Sïl est peu abondant, il est en général, disséminé d'une façon uniforme, à l'état de fines granulations, dans toute la masse. Au contraire, dans les œufs qui en possèdent beaucoup, il tend à se séparer du cytoplasme. Ainsi, dans l'œuf des Oiseaux, le cytoplasme est localisé sous forme d'un petit disque à un póle de l'œuf (fig. 2); tout le reste de la masse du jaune est du vitellus. Dans les œufs des Insectes (fig. 3), le cytoplasme $c$ forme une couche périphérique, et le vitellus $v$ est accumulé rers le centre. L'œuf paurre en vitellus se divise en totalité pour produire l'embryon; sa segmentation est dite totale. Au contraire dans les gros œufs, tels que ceux des Oiseaux, seul le disque cytoplasmique proprement dit évolue en l'embryon et le vitellus est une masse inerte qui est peu à peu digérée. La segmentation est

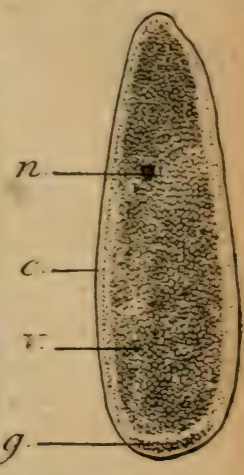

Fig. 3. - Ovule d'Insecto chrysomélide /Calligrapha multipunctata). $c$, cytoplasma périphérque; $v$, vitellus; $n$, noyau; $g$, granules du póle postérieur nécessaires à la formation des cellules génitales (d'après Hegner). partielle. Entre ces deux cas extrêmes existent de nombreux termes intermédiaires.

On devine aisément, à ces quelques indications, l'énorme diversité de structure des ovules; il n'est ni nécessaire, ni possible de s'y arrêter ici. Sous cette diversité se cache un fait général et capital : l'ovule est toujours une cellule, dont on aperçoit d'ailleurs aisément, au moins jusqu'au voisinage de la maturité, le noyau vésiculeux et turgescent. 
§ 2. - Le spermatozoïde, ou gamète mâle. - Le spermatozoïde ou gamète màle nous montre des caractères opposés à l'ovule : réduction de la masse, absence totale de réserres, grande mobilité, alors que l'ovule est inerte.

A ce contraste s'en associe un autre, celui du nombre. La ponte de certains animaux se monte, il est vrai, ̀̀ un chiffre élevé d'ovules (celle de la morue,

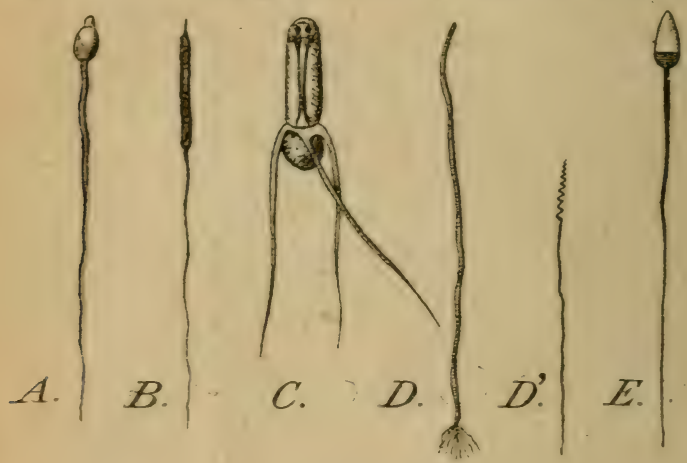

FiG. 4. - Spermatozoides; forme générale d'après le vivant.

A. Honme; B, Ver de terie; C. Homard; $D$ et $D^{\prime}$, les deux formes de spermatozofles de la Paludine (Mollusque) : $D$, sp. vermiforme ou oligopijrene; $D^{\prime}$ spermatozoide filiforme ou eupyrène, of. p. 13); $E$, Oursin. Girossissement de 700 à 1.200 .

par exemple, est estimée à 4 millions); mais la plupart des espèces n'en mûrissent qu'un nombre très restreint; on citerait même aisément des exemples où la femelle, dans toute son existence, ne pond qu'un œuf unique. Pour prendre le cas de l'espèce humaine, on estime a une cinquantaine de mille le total des ovules renfermés dans les oraires d'une femme, et quelques centaines seulement arriveront à maturité. 
Au contraire, les spermatozoïdes sont fabriqués en nombres colossaux. Un millimètre cube de sperme humain en contient environ 60.000 et on évalue qu'il en est émis 200 millions à la fois.

En raison de sa petitesse, le spermatozoïde ne peut être étudié qu'aux forts grossissements du microscope et s'il a été aperçu dès la fin du xvı1 siècle, sa connaissance précise est toute récente. Chez la plupart des animaux, les spermatozoïdes sont de petits corps filiformes (fig. 4), présentant un renflement antérieur, improprement appelé la tête, et un longr filament extrêmement fin, dit la queue; à la jonction de ces deux parties, on distingue, par des procédés appropriés, une portion différenciée, dite la pièce intermédiaire ${ }^{1}$.

Exceptionnellement toutefois, les spermatozoïdes ont des formes très différentes, comme c'est le cas chez les-Crustacés (homards, langoustes, [fig. 4C] écrevisses, crabes, etc...) ou les Vers nématodes.

Une étude minutieuse, telle qu'elle a pu être faite en ces dernières années, a montré que les spermatozoïdes avaient en réalité une structure compliquée et extrèmement variée dans le détail, suivant les espèces. Cette variété n'est pas moindre que celle des ovules. Elle lui correspond; il y a une adaptation réciproque des ovules et des spermatozoïdes et, chez beaucoup

1. Quelques données numériques, évaluées en millièmes de millimètre, fixeront les idẻes sur les dimensions de ces éléments :

\begin{tabular}{|c|c|c|c|c|c|}
\hline \multirow{2}{*}{ ESPÈCES } & \multirow{2}{*}{$\begin{array}{l}\text { Longueur } \\
\text { totale du } \\
\text { spermatozoide }\end{array}$} & \multicolumn{2}{|c|}{ T Eे TE } & \multirow{2}{*}{$\begin{array}{l}\text { Longueur } \\
\text { de la pièce in- } \\
\text { termédiaire }\end{array}$} & \multirow{2}{*}{$\begin{array}{l}\text { Longueur } \\
\text { de la } \\
\text { queue }\end{array}$} \\
\hline & & Longueur & Largeur & & \\
\hline Homme. . . & 60 & 4 & $2-3$ & 6 & 50 \\
\hline Rat.... & $170-200$ & 10 & & 55 & $100-120$ \\
\hline Pinson. . . & 250 & & & & \\
\hline Axolotl. . . & 400 & 120 & & & \\
\hline Grenouille & 70 & 15 & & & 50 \\
\hline Raie, , . & 215 & 50 & & 15 & 150 \\
\hline
\end{tabular}


d'animaux, les particularités de structure des seconds sont manifestement en rapport avec les conditions ${ }^{7}$ de leur pénétration dans les premiers. La tête sera tantôt unc masse ellipsoïdale comme chez l'Homme; tantôt une lame à courbures complexes comme chez le Rat

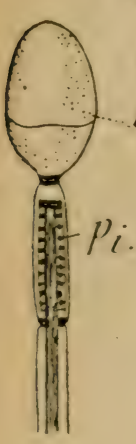

A.

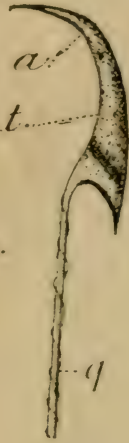

B.

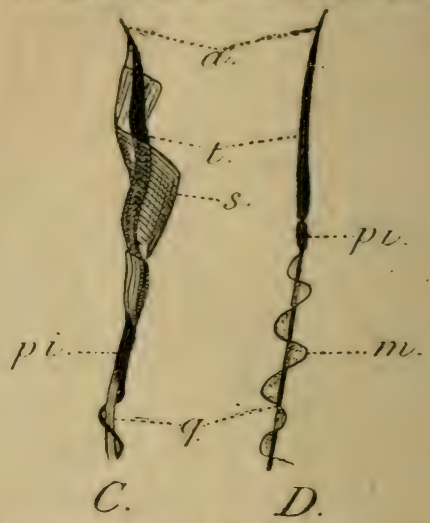

Fig. 5. - Extrémité antérieure de quelques spermatozoldes:

A, Homme (schéma d'après Waldeyer); $B$, Rat (la tête en forme de spatule creuse est vue de profil); $C$, Oiseau (la tête est entourée d'une membrane spirale s; $D$, Triton (la queue est munie d'une membrane ondulante $m$ ). ( $B, C, D$, imitees de Ballowitz).

$t$, tete; $p, i$, pièce intermediaire; $q$, queue; $a$, acrosome ou perforatorium.

et divers Rongeurs; tantôt une longue pointe légèrement spiralée et entourée d'une collerette comme chez beaucoup d'Oiseaux; tantôt elle se décompose nettement en deux parties : la tète proprement dite, massive, et un organe de pénétration acéré (qui a reçu des noms divers, perforatoriuin, acrosome, etc.), fine pointe ou hameçon. La pièce intermédiaire et la queue offrent elles aussi des particularités de structure multiples auxquelles je dois ici me borner à faire allusion. Tous les spermatozoïdes d'un mème animal sont 
semblables. Cependant certaines espèces en produisent de deux types distincts différant profondément par la taille, la forme, la structure et la genèse. Ce dimorphisme, qui a donné lieu à des recherches nombreuses et remarquables, sans qu'on ait pu jusqu'ici en trouver une interprétation satisfaisante, existe par exemple, d'une façon très marquée, chez beaucoup de Mollusques appartenant à l'ordre des Gastéropodes Prosobranches et en particulier chez la Paludine, espèce très commune dans nos fleuves; la figure $4 D, D^{\prime}$ en donnera une idée.

Enfin si, chez la plupart des animaux, les spermatozoïdes sont librement mobiles dans la liqueur séminale, chez d'autres ils sont agglomérés par paquets, dans des appareils de formes très compliquées et très variées, ainsi qu'en témoignela figure6, et qu'on appelle des spermatophores Ces dispositions sont en rapport avec la façon dont s'accomplit la rencontre des gamètes.

Comme l'ovule, le spermatozoïde est une cellule. Mais cela ne résulte pas immédiatement de sa structure défini-

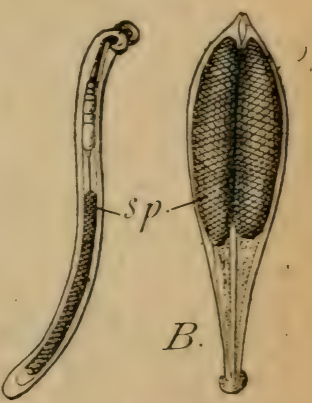

Fig. 6. - Deux exemples de spermatophores : $A$, de Seiche; $B$, de Sangsue (Glossosiphonia, d'après Brumpt). $S p$. pocle renfermant les spermatozoides. tive. C'est l'étude de sa genèse qui en fournit la preuve. Nous verrons par elle que la tête du spermatozoïde est constituée à peu près en totalité par le noyau cellulaire. Le cytoplasme extranucléaire de la cellule est réduit à une masse insignifante qui forme la queue et des portions différenciées de la tète. 


\section{CHAPITRE II}

\section{LA GENĖSE DES GAMĖTES}

Le tissu germinal. - Son origine chez l'embryon. - Weismann et la distinction du soma et du germen. - Différenciation généralement très précoce du tissu germinal.

Ovogénèse. - Ovules et cellules folliculaires. - Nutrition de l'ovule.

Spermatogénèse. - Spermatogonies et cellules nourricières.

- Le spermatozoïde, comme l'ovule, est une cellule.

Les données précédentes sur les gamètes à leur état définitif doivent ètre complétées par une étude sommaire des stades qui précèdent, c'est-à-dire de l'ovogénèse et de la spermatogénèse; mais il ne peut être question ici que d'en situer les points essentiels.

§ 1. - Tissu germinal, gonades. - Et tout d'abord, orules et spermatozoildes se forment (au moins chez la généralité des animaux) dans des organes bien définis, les glandes génitales ou gonades : ovaires, testicules, ou glandes mixtes produisant à la fois.les deux catégories de gamètes.

Il y a donc, dans l'organisme, un tissu reproducteur ou germinal. Plus l'embryologie progresse et plus tôt on trouve la trace de ce tissu chez l'embryon. Parfois même, comme nous allons le voir, on saisit celle-ci dès le début même du développement. Dès que ce tissu est différencié, il a, d'une façon défini- 
tive, - au moins dans la généralité des cas — le monopole de la reproduction. Sa suppression, même aux stades les plus précoces, entraine le plus souvent la stérilité définitive de l'individu; il ne peut pas être régénéré. De là est née une idée, qui a pris dans la biologie contemporaine une place très grande et a revêtu les allures d'un dogme dont la base est cependant des plus contestables : celle de l'autonomie du tissu germinal par rapport au reste de l'organisme; ce serait comme un organisme particulier dans l'organisme général.

Soma et germen. - G'est Weismann qui a surtout déreloppé cette conception, sous le nom de théorie du plasma germinatif et qui a isolé l'un de l'autre et opposé l'un à l'autre le corps ou soma et le tissu reproducteur ou germen. Au cours du développement. à partir de l'œuf, les divers tissus se spécialiseraient. progressivement, tandis qu'une lignée cellulaire privilégiée conserverait intégralement la totalité des propriétés de l'œuf; c'est elle qui donnerait naissance aux glandes génitales; elle constituerait le germen, continu ainsi de génération en génération. Les générations successives ne seraient que la différenciation d'une série de somas, servant simplement d'enveloppes à une lignée germinale unique et immortelle.

Je ne veux pas discuter ici cette conception, née de spéculations théoriques plus que d'observations positives et qui, a priori, est très discutable au point de vue physiologique.

\$ 2. - Origine du tissu germinal chez l'individu. - Elle nous intéresse en ce qu'elle a contribué, pour une part, à faire rechercher, à des stades de plus en plus précoces du développement, la première ébauche des glandes génitales: or les obserrations récentes sur des groupes d'animaux très divers montrent 
qu'effectivement les cellules-souches de ces glandes se différencient de fort bonne heure.

Chez un Ver Nématode, qui a été un des principaux thèmes de recherches cytologiques, l'Ascaris megalocephala, à partir de la première division de l'œuf, Boveri a pu discerner une lignée de cellules se comportant d'une façon spéciale; or, cette lignée est l'origine des glandes génitales de l'Ascaris. On peut donc dire que celles-ci sont, en réalité, différenciées du soma, chez l'Ascaris, dès le début même du développement de l'individu.

Chez des Insectes de divers groupes, on a fait des constatations analogues. Par exemple, chez les Diptères (Mouches, Cécidomyies, Chironomides, etc.), les cellules génitales primordiales se distinguent presque dès le début du développement de l'œuf. Dans les Coléoptères de la famille des Chrysomélides, il en est encore ainsi. Je signalerai, à cet égard, d'intéressantes expériences dues à Hegner. Chez la Chrysomèle de la pomme de terre (Leptinotarsa decemlineata) et les formes voisines, l'étude histologique de l'œuf non encore segmenté montre, au pôle postérieur, une masse de granulations qui se distingue par des réactions colorantes spéciales (fig. $3, g$ ). Ces granulations passent dans des cellules qui s'isolent tout au début du développement et qui sont l'origine des glandes génitales. Si on supprime ces cellules et même si on détruit, dans l'œưf non segmenté, la région du pôle où siégeaient les granulations en question, on obtient des individus dépourvus de glandes génitales.

On pourrait citer des observations de même ordre relatives aux Crustacés, et même aux Vertébrés ${ }^{1}$, indiquant une différenciation extrêmement précoce de la

1. Notamment sur les Raies (Beard), sur les Lamproies (Wheeler), sur les Truites et les Grenouilles (Nussbaum) et meme sur des Reptiles et des Mammifères. 
lignée cellulaire qui fournit les glandes génitales de l'individu.

Il est donc bien exact, en fait, que le tissu germinal se différencie souvent de fort bonne heure, parfois mème dès les débuts de la segmentation de l'œuf. L'originedes gamètes peut donc ètré poursuivie jusqu'aux premières phases de l'embryogénie, et c'est ce que nous retenons ici, sans pour cela y voir une loi générale, ni adhérer aux conceptions théoriques de Weismann. Si, en effet, elles étaient justifiées, elles devraient être d'une généralité complète; or, chez les végétaux, chez les animaux bourgeonnants, on ne peut pas dire que le tissu sexuel forme une lignée distincte dès le début du développement. Il ne se différencie que d'une façon tardive, et on peut le faire apparaître, en provoquant des phénomènes de régénération, dans les points les plus variés. On ne peut, sans forcer les faits, voir, dans ces cas, une indépendance réelle du soma et du germen.

§3. - La différenciation des gamètes. - Ovogénèse et spermatogénèse. - La formation des gamètes dans la glande génitale, c'est-à-dire l'ovogénèse ou la spermatogénèse, est aujourd'hui bien connue. Ces processus ont été étudiés arec une extrème minutie. Voyons-en les grandes lignes.

Il faut remarquer tout d'abord que les premiers stades de la différenciation d'une glande génitale ne permettent pas de dire si elle produira des ovules ou des spermatozoïdes. On ne peut savoir, même à un examen histologique précis, si elle est l'ébauche d'un testicule ou d'un ovaire.

Ovogénèse. - Quand il s'agit d'un ovaire, une partie des cellules composant l'organe acquièrent, à partird'un certain moment, un rolume nettement plus considérable et un gros noyau. Ce sont des ovulesdifférenciés 
et qui, dès lors, ne se multiplieront plus par division. Ils subiront seulement, plus ou moins rapidement, une croissance, au cours de laquelle ils élaboreront leur vitellus et acquerrontleur taille définitive. Certains animaux produisent des ovules toute leur vie; mais, chez d'autres, cela n'a lieu qu'au début de l'existence : ce dernier cas est celui des Mammifères. Chez la femme, par exemple, tous les ovules sont formés définitivement quelques mois après la naissance; un petit nombre d'entre eux mûriront successivement, à partir de l'époque de la puberté.

Les cellules d'un oraire qui deviennent des ovules sont la minorité. A chaque ovule sont associées presque toujours des cellules plus ou moins nombreuses; gardant les caractères de simples éléments épithéliaux et qui forment autour de lui une enveloppe plus ou moins complexe, à laquelle on a donné le nom de follicule: elles sont dites cellules folliculaires (V. fig. 1). Leur activité fonctionnelle concourt, direc-

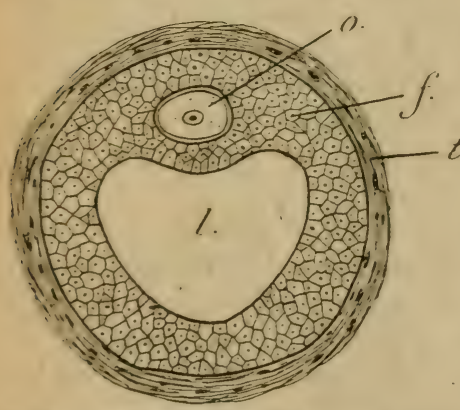

Fic. 7. - Follicule de de Graaf à peu près mur. $o$, ovule; $f$, cellutes folliculaires; $l$, cavité remplie de liquide (liquor folliculi); $t$, enveloppe conjonctive ou thèque du follicule.

tement ou indirectement, au phénomène essentiel de la croissance de l'ovule, l'élaboration du vitellus.

Cette coopération est un phénomène général, mais susceptible de modes extrêmement variés, et je me borne à préciser les indications précédentes par l'exemple du follicule des Mammifères.

Il est formé par un grand nombre de couches cellulaires, au centre des- 
quelles on roit l'orule proprement dit, baigné par un liquide (liquor folliculi), sécrété par les cellules folliculaires $f$ (fig. 7 ). Le tout est enveloppé de cellules conjonctives constituant la thèque. Un follicule est donc un ensemble très complexe et assez gros pour ètre visible à l'œil nu. C'est lui qu'on a cru d'abord être l'œuf humain, et on l'appelle encore souvent aujourd'hui vésicule de de Graaf, du nom de l'anatomiste hollandais qui l'avait, le premier, décrit au $\mathrm{xvII}^{\mathrm{e}}$ siècle. C'est seulement au début du $\mathrm{xIx}^{\mathrm{e}}$ siècle que l'ovule véritable a été discerné dans le follicule.

Chez divers animaux où les formations folliculaires sont très réduites, l'accumulation du vitellus dans l'ovule est assurée d'une autré façon. Il se forme tout d'abord un grand nombre d'orules. Mais quelques-uns d'entre eux seulement atteignentla maturité en dévorant leurs frères, dont ils assimilent la substance à un stade

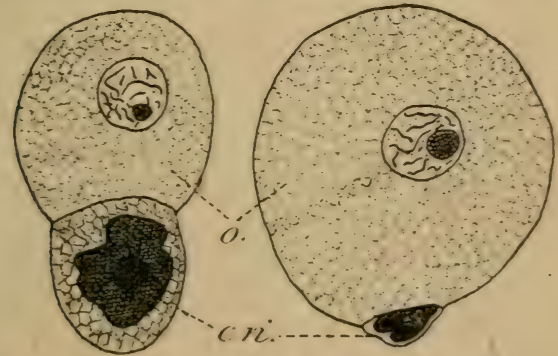

Fis. S. - Orule o et cellule sueur, satellite et nourriciere $n$ d'Oplwyotrocha Annélide); à gauche, stade jeune; à droite, stade plus avancé; Tovule s'est développé, la cellule nourricière s'est atrophiée (d'après horscheit).

plus ou mois arancé de leur évolution (fig. 8). Ici encore le phénomène est susceptible de beaucoup de formes particulières ${ }^{1}$.

1. Parfois chaque orule n'absorbe qu'un seul élément satellite. Parfois il en absorbe un grand nombre. Chez de petits Crus- 
Sans entrer dans plus de détails, nous voyons que l'élaboration de quantités relativement considérables de substances vitellines dans l'ovule est le résultat de la concentration, dans une cellule particulière élue, des résultats de l'activité fonctionnelle d'un plus ou moins grand nombre d'éléments satellites.

Quand l'ovule a atteint sa maturité, et s'est détaché de l'ovaire, il subit une dernière série de transformations, terminant l'orogénèse, et que l'on a appelées assez improprement les phénomènes de maturation. Ils consistent dans l'expulsion de deux petits corps appelés les globules polaires. Mais comme ce phénomène a son équivalent dans la spermatogénèse, nous l'étudierons plus loin simultanément pour les deux catégories de gamètes.

Spermatogénèse. - La spermatogénèse offre un tableau tout différent du précédent. Ici, toutes les cellules restent petites et se multiplient actirement pour produire le nombre énorme des spermatozoïdes. Elles se disposent en fins canalicules, les tubes séminifères, dont l'ensemble réalise, à l'intérieur de l'organe, une très grande surface épithéliale toute tapissée de cellules appelées spermatogonies; chacune de cellesci est la souche de nombreux spermatozoïdes.

Cependant, il y a, dans l'épithélium des tubes séminifères, une division du travail analogue à celle qui est réalisée dans l'ovaire, entre les ovules et les cellules folliculaires. A côté des spermatogonies, en effet, il y a des cellules qui ne donneront jamais naissance à des spermatozoïdes, mais qui jouent, pour la lignée séminale, le rôle d'éléments de soutien et aussi d'éléments nourriciers. Les minutieuses études des der-

tacés d'eau douce du groupe des Cladocères, les Mö̈na, dans la formation des œufs d'hiver, chaque ovule définitif a absorbé ainsi 47 ovules frères. 
nières années ont montré, en effet, qu'elles élaborent des substances spéciales; autour d'elles, viennent, ¿ certaines phases, se grouper, en faisceaux serrés, les spermatozoïdes en formation. Sous des formes diverses, on trouve, dans les testicules des animaux de presque tous les groupes, cette association des cellules nourricières et de la lignée séminale proprement dite. Chez les Vertébrés supérieurs, les Oiseaux et les Mammifères, ces éléments nourriciers ont été particulièrement bien étudiés et on les désigne généralement du nom de Sertoli qui les a, le premier, nettement discernés.

Ces deux catégories de cellules (lignée séminale et éléments sertoliens) réagissent d'ailleurs de façons toutes différentes à de multiples actions. Dans une série de malformations des testicules chez les Mammifères1, la lignée séminale avorte complètement, tandis que les éléments sertoliens subsistent intacts. Par l'action convenablement conduite des rayons $\mathrm{X}$, divers auteurs ont pu détruire complètement et définitivement tous les éléments séminaux proprement dits, stériliser ainsi totalement un testicule, tandis que les éléments de Sertoli subsistaient et constituaient désormais seuls la paroi des tubes séminifères 2 .

Les spermatozoïdes sont l'aboutissement terminal

1. Testicules ectopiques, cryptorchidie.

2. Les indications très succinctes qui précèdent mettent en évidence dans l'ovaire et le testicule deux catégories de cellules: la lignée des gamètes proprement dite et les cellules nourricières, cellules folliculaires ou élẻments sertoliens. L'ensemble de ces deux catégories forme la glande génitale ou gonade proprement dite. Mais, très souvent (chez les Vertébrés par exemple), ils sont enchâssés en quelque sorte dans une trame formée par des cellules somatiques qui remplissent les interstices entre les follicules ou les tubes séminifères. Ce tissu de remplissage (ou interstitiel) joue un rôle important dans diverses manifestations de la Sexualité, ainsi que nous le verrons, et pour cette raison il doit être mentionné ici. 
d'urie série de générations cellulaires se succédant dans les tubes séminifères à partir des spermatogonies, mais ve sont eux-mêmes des cellules; en effet, ils résultent de la transformation directe de la dernière génération de la lignée séminale à laquelle on donne le nom de spermatides (fig. 9). Les spermatides

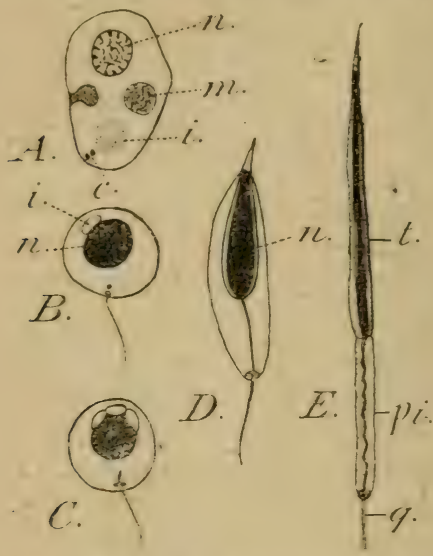

Fic. 9. - Transformation de la sprermatide en spermatozoide.

$\boldsymbol{A}$, la spermatide et ses diverses différenciations (schéma d'après Meves); $\boldsymbol{B}-\boldsymbol{E}$, ses transformations chez les Sélaciens (Poissons) (d'après Suzuki et Hermann).

$n$, noỵau; $m$, mitochondries; $i$, idiosome; $c$, centrosomes (d'oú part le filament caudal); $p i$, pièce intermédiaire; $q$, queue.

sont bien reconnaissables comme cellules; elles sont sphériques et ont un noyau résiculeux $(A)$. Ce noyau s'allunge et s'étire $(D, E)$; une forte partie du cytoplasme est sourent rejetée; une autre partie, caractérisée par des réactions spéciales, vient coiffer antérieurement le noyau allongé et forme l'appareil perforateur antérieur du spermatozoïde (acrosome) De l'extrémité postérieure du noyau part et s'allonge graduellement 
le filament caudal $(B, C)$; on a reconnu qu'il naît à partir de deux grains appartenant au cytoplasme de la spermatide, les centrosomes. Autour de ces centrosomes, une portion du cytoplasme de la spermatide forme la pièce intermédiaire $(E, p i)^{1}$.

Ces quelques données suffisent à établir que, malgré son allure très spéciale, le spermatozoïde est une cellule, tout comme l'ovule, mais dont la masse cytoplasmique est extrêmement réduite.

1. Là, s'accumulent en particulier des granulations ayant des réactions propres vis-à-vis des matières colorantes et auxquelles les cytologistes ont donné le nom de mitochondries. 


\section{CHAPITRE III}

\section{LA MATURATION DES GAMĖTES ET LA FÉCONDATION}

La phase de réduction chromatique terminant l'ovogénèse et la spermatogénèse. - Ovocytes et spermatocytes. - Réduction à moitié (méiose) du nombre des chromosomes.

La fusion des gamètes ou fécondation. - Pénétration d'un seul spermatozoïde dans l'ovule (monospermie). - La fécondation double, dans l'œuf, le nombre des chromosomes existant dans chacun des gamètes. - Égalité d'apports paternel et maternel en chromatine.

Les chromosomes doivent-ils être considérés comme le support matériel et spécifique des propriétés héréditaires?

§1. - La phase méiotique dans l'ovogénèse et la spermatogénèse. - Les deux gamètes, spermatozoïde et ovule, sont donc, l'un comme l'autre, une cellule, c'est-à-dire des éléments morphologiquement équivalents. Mais l'étude attentive de leur formation a mis en évidence, entre eux, une équivalence plus précise encore et qui s'applique aux deux règnes, végétal et animal. Je me bornerai ici à l'indiquer sommairement.

Quand une cellule quelconque se divise (fig. 10), le noyau semble disparaître; sa membrane se dissout, son contenu se confond avec le cytoplasme cellulaire, et il n'est représenté que par des filaments qui se teignent fortement par certaines matières colorantes ${ }^{1}$. I.6

1. Dites basiques; ex. : l'hématoxyline. 
substance de ces filaments a reçu des biologistes, en raison de cette propriété, le nom de chromatine et les filaments eux-mêmes celui de chromosomes. On assiste à un cycle de processus très complexe, qui est désigné sous les noms de caryocinèse ou mitose. Chez une espèce

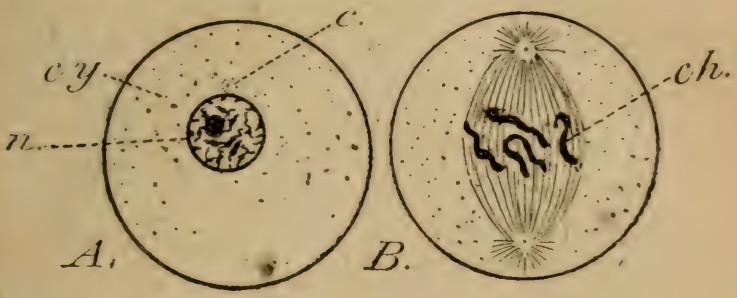

Fic. 10. - Cellule au repos (A) et en caryocinèse ( $B$ ) (schémas): $n$, noyau; $c$, centrosomes; $c h$, chromosomes; cy, cytoplasme.

donnée, animale ou végétale, le nombre des chromosomes qui apparaissent, lors des divisions cellulaires, dans les divers tissus, est constant. Nous le désignerons par' $2 n$.

Or l'ovule, au moment où il est fécondé ou immédiatement avant, expulse, à sa périphérie, deux petits corpuscules, désignés sous le nom de globules polaires (fig. 11); et l'on a reconnu que cette expulsion était le résultat de deux divisions cellulaires se succédant rapidement. A chacune de ces divisions, l'une des cellules formées est très petite : c'est un globule polaire; l'autre, l'ovule, retient toute la masse du cytoplasme. C'est seulement après l'expulsion des globules polaires, c'est-à-dire après cette double division que la fécondation véritable peut s'accomplir, que l'ovule est physiologiquement mûr; aussi appelle-t-on souvent ce phénomène, la maturation ou les divisions maturatives de l'ovule. C'est le terme de l'ovogénèse; cette phase peut sembler, au premier abord, insigni- 
fiante, mais on a été conduit à y attacher une grande importance.

On a constaté, en effet, dans d'innombrables especes appartenant aux groupes les plus variés du

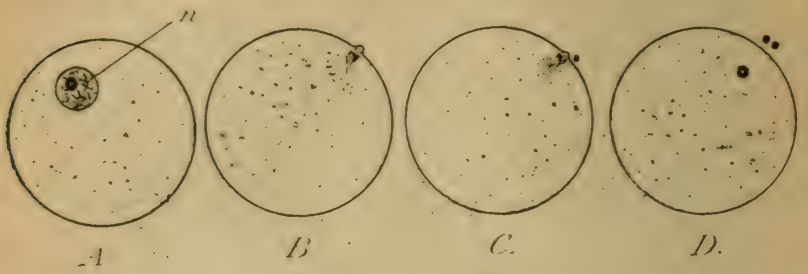

TiG. 11. - Formation des giobules poluires, ou maluration de lovule (schéma)

$A$, orule arant la maturation . $n$, noyau ou vésicule germinative; $B$, expulsion du premier globule polaire (figure caryocinétique périphérique); $C$, expulsion du second; le premier est visible hors de l'orule; $D$, ovule mûr (avec le noyau réduit ou pronucleus femelle).

règne animal, que, lors des deux divisions cellulaires donnant naissance aux globules polaires, le nombre des chromosomes est réduit de moitié. De $2 n$, il passe à $n$. S'il y arait 24 chromosomes. lors des divisions cellulaires dans les divers organes, on n'en trouve que 12 dans la formation des globules polaires. Nous dirons qu'il y a eu réduction chromatique ou méiose 1 .

D'autre part, l'étude de la spermatogénèse a mis en évidence une réduction parallèle du nombre des chromosomes de $2 n$ à $n$, lors des deux dernières divisions cellulaires qui aboutissent à la formation des spermatides.

Il y a là une similitude entre la spermatogénèse et l'orogénèse, dont on ne peut manquer d'ètre frappé et dont la généralité, surabondamment reconnue, atteste l'importance. Les deux divisions maturatives de

1. De $\mu$ sเwน, moins. 
l'ovule (formation des globules polaires), c'est-à-dire les deux dernières divisions de l'ovogénèse, équivalent donc aux deux dernières divisions de la spermatogénèse, d'où résultent les spermatides. On a exprimé ce parallélisme en appelant ovocyte l'ovule arant l'expulsion des globules polaires, et spermatocyte ou auxocyte la génération des cellules qui, dans la spermatogénèse, en se divisant deux fois encore, produiront les spermatides. Ovocyte et auxocyte se correspondent en vertu des phénomènes résumés plus haut et que traduit le tableau ci-dessous (fig. 12):

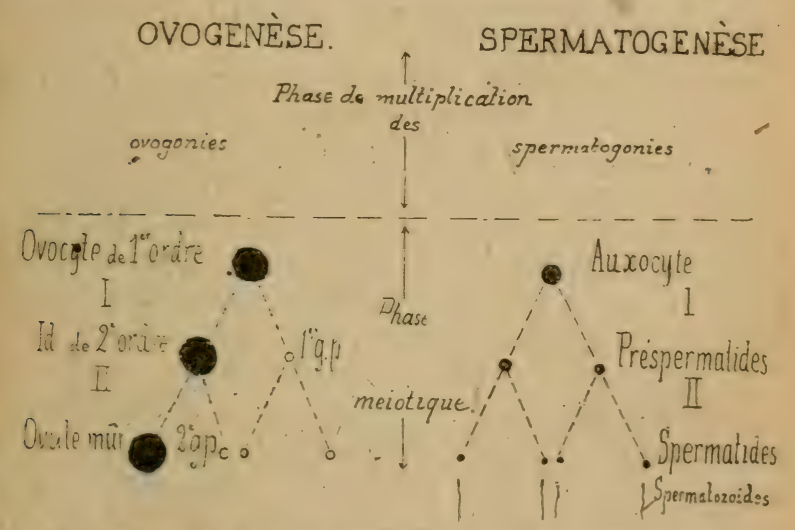

Fig. 12. - Tableatl le la corresjondance de la phase miotique dans l'ovogénèse et la spermatogénèse.

Les deux divisions cellulaires en question sont caractérisées par une réduction de moitié du nombre des chromosomes; on les a heureusement désignées sous le nom de phase méiotique ${ }^{1}$.

1. La description précédente ne s'applique qu'aux animaux dits Métazoaires. Pour les Protozoaires et les Végétaux, voir la sixième partie, p. 269. 
§ 2. - La fusion des gamètes ou fécondation. Quelques mots sur la fusion des gamètes, c'est-àdire sur la fécondation, sont nécessaires pour compléter l'histoire de ces éléments constitutifs de la sexualité et feront au reste mieux comprendre l'importance de certains des points mentionnés précédemment.

La fécondation est un phénomène connu aujourd'hui dans ses détails, chez un très grand nombre d'animaux et de végétaux appartenant aux groupes les plus variés. Ses traits fondamentaux offrent une homogénéité parfaite dans les deux règnes. Les différences sont secondaires.

Chez les animaux, tantôt les gamètes sont l'un et l'autre rejetés au dehors et alors leur rencontre, la fécondation, a lieu dans le milieu extérieur; tantôt les ovules, même après s'être détachés de l'ovaire, séjournent plus ou moins longtemps dans les voies génitales de la femelle et y rencontrent les spermatozoïdes que le mâle a intraduits dans celles-ci : la fécondation est alors interne.

C'est dans le premier cas, plus aisé à observer, que la fécondation a été étudiée tout d'abord, chez les Echinodermes (Oursins, Etoiles de mer). Elle y a été connue, dans ses grands traits, de 1875 à $1880^{1}$. Aujourd'hui encore, les Echinodermes restent l'exemple classique pour assister aisément à la pénétration du spermatozoïde dans l'ovule. Il suffit, pour cela, de mélanger, dans une goutte d'eaú placée sous le microscope, des ovules et des spermatozoïdes prélevés dans des glandes génitales mûres. On voit, au bout de quelques instants, chaque ovule entouré d'une auréole de spermatozoïdes (fig. 13), comme si ceux-ci

1. Elle avait étẻ observée antérieurement, chez les Algues, notamment, dès 1854, chez les Fucus, par les belles observations de Thuret. V. p. 301. 
étaient attirés par celui-là. Chez certaines Fougères, Pfeffer a montré qu'il y avait effectivement une attraction d'ordre chimique, ou, comme on dit, un phénomène de chimiotaxie positive : les spermatozoïdes ou anthérozoïdes sont attirés, dans ce cas, en effet, par l'acide malique. Chez les animaux, et en particulier chez les Oursins, on n'a pas réussi à mettre

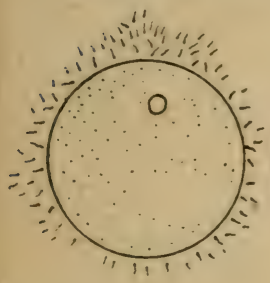

$-1$

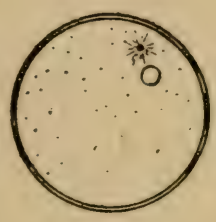

l'.

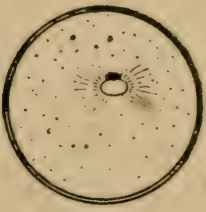

C.

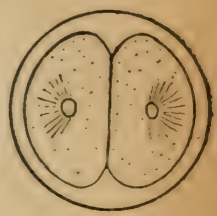

1).

Fig. 13. - Fécondation de l'œuf et sa première division (schémas).

$A$, l'ovule entouré de spermatozoïdes (à son intérieur le noyau ou pronucleus femelle); $B$, le spermatozoide a pénétré dans l'ovule qui s'est entouré d'une membrane; des radiations (aster) se dessinent autour du noyau du sperma. tozoïde (pronucleus mâle); $C$, fusion des deux pronuclei; $D$, l'œuf divisé en deux cellules.

nettement en évidence une action chimique précise; elle a même été niée, mais le dernier mot ne semble pas dit à ce sujet.

L'un des spermatozoïdes, placé plus favorablemenc, pénètre dans le cytoplasme ovulaire, qui semble former, vers lui, à ce moment comme un cône d'attraction (fig. 14); la tête seule pénètre en général et, presque immédiatement après, on voit se manifester autour de l'ovule jusque-là nu, une membrane transparente qui barre la route à tous les autres. Dans les conditions normales, un seul spermatozoïde pénètre ainsi dans l'ovule; la fécondation est dite monospermique; la pénétration de plusieurs spermatozoïdes, ou 
polyspermie, ne se produit que dans des conditions pathologiques ${ }^{1}$.

L'orule fécondé est devenu l'œuf (fig. 13). Autour du spermatozoïde, se dessine une figure rayonnante, l'aster. Elle se déplace vers le centre, se dédouble et l'on assiste à la série des phases d'une division cellulaire ou caryocinèse, qui partage l'œuf en deux cellules égales, les deux premières de l'embryon. Chacune

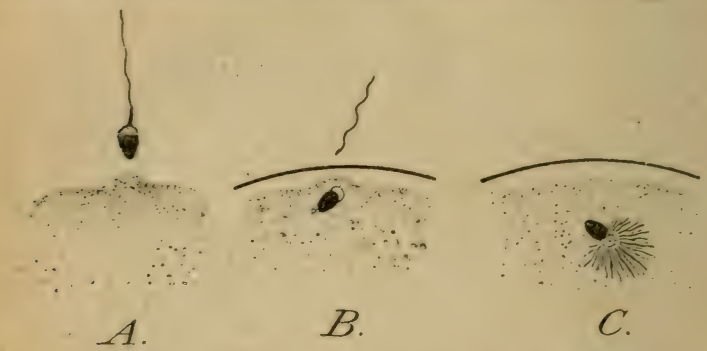

Fig. 14. - Pénétration du spermatozoide dans l'ovule.

4 , cone d'attraction en face du spermatozoide, à la surface de l'ovule; $B, 1 a$ tête du spermatozơde a pénétré; $C$, formation de l'aster.

d'elles se divise à nouveau et ainsi de suite. Tout le déreloppement n'est qu'une succession de divisions cellulaires.

Grâce aux méthodes modernes de l'histologie, on a $\mathrm{pu}$, en faisant agir des matières colorantes sur l'œuf préalablement tué par des réactifs dits fixateurs, suivre les transformations successives du noyau de l'orule et de celui du spermatozoïde rlans les phénomènes précédents. On a vu ainsi que les noyaux des

1. Il faut faire une restriction pour les œufs volumineux, comme ceux des Oiseaux, des Reptiles, des Raies et des Requins. des Poulpes et des Seiches. Dans ces ceufs, une forte polyspermie est la règle; mais un seul des spermatozoïdes qui ont pénśtré joue véritablement le rôle fécondateur. 
deux gamètes se fusiomment en un noyau mixte, de façon que les deux premiers noyaux de l'embryon et par suite tous les autres qui en dérivent, sont constitués d'une façon sỵmétrique par les substances nucléaires paternelle et maternelle.

Mais c'est sur d'autres types que les Echinodermes, plus favorables parce qu'ils possèdent des chromosomes olus grands et moins nombreux, que ces phé-
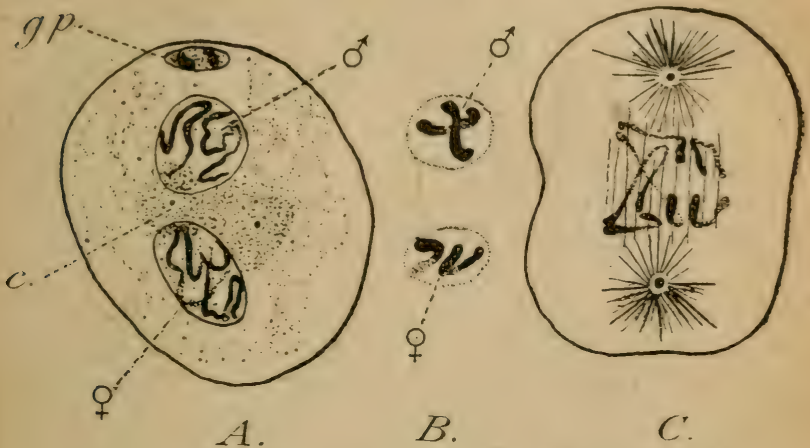

Fic. 15. - Stade de ia fécondation de l'Ascaris megalocephala montrant le: formation et la répartition des chromosomes paternels et maternels (diaprès Boveri).

$c$, centrosomes: $y p$. globule polaire; $o^{\top}$ et $q$, les deux pronuclei (chacun d'eux produit deux chromosomes $(B)$ et chacun de ces chromosomes se divise longitudinalement en deux moitiés qui se portent vers les deux pôles de la caryocinese $(C)$, pour former les noyaux des deux premieres cellules de l'embryon).

nomènes ont pu èire exactement analysés. C'est l'Ascaris megalocephala, ver nématode, parasite dans l'intestin du cheral, qui en a livré le secret, grâce aux recherches d'Ed. van Beneden, en 1883.

§3. - La fécondation compense la méiose. - Après la pénétration du spermatozoïde et l'expulsion des globules polaires, le noyau de chaque gamète devient dans 
l'œuf une vésicule qui subit toutes les transformations d'un noyau en division (fig. 15). Chaque noyau gamétique ne donne naissance, chez l'Ascaris, qu'à deux chromosomes de grande taille, par suite aisés à étudier.

Ces chromosomes, dans chaque noyau, se divisent longitudinalement. Il y en a par suite quatre d'origine paternelle et quatre d'origine maternelle. Au moment où l'œuf se divise en deux cellules, le noyau de chacune d'elles se reconstitue aux dépens de deux chromosomes paternels et de deux chromosomes maternels. C'est-à-dire que les noyaux des deux premières cellules de l'embryon (et par suite de tous les tissus ultérieurs qui en dérivent) sont, quant à leur chromatine, de nature mixte; ils ont reçu, en quantités égales, sous forme de chromosomes, de la substance chromatique d'origine paternelle et d'origine maternelle.

Le phénomène de la fécondation - et ceci a été reconnu général, chez les régétaux comme chez les animaux - nous traduit donc, sous une forme matérielle, dans la constitution des noyaux primordiaux de l'embryon, l'apport équivalent et la fusion intime de la substance même des deux progéniteurs. C'est, tout au moins, une représentation matérielle frappante de la combinaison des propriétés héréditaires paternelle et maternelle.

Notons aussi que, dans l'œuf qui se divise en deux cellules, et - comme l'observation l'a appris - dans toutes les cellules qui en dérivent, le nombre des chromosomes, préalablement réduit à $n$, pour chaque gamète, lors de la phase méiotique se trouve ramené à $2 n$. Ainsi, dans l'Ascaris megalocephala, chaque gamète apporte 2 chromosomes; les deux cellules issues de l'œuf en reçoirent, d'après le mécanisme ci-dessus décrit, et en transmettent 4. La fécondation compense donc la méiose; elle rétablit le nombre $2 n$ 
de chromosomes que la méiose avait réduit à $n$. La méiose nous apparaît donc comme un mécanisme qui, en ramenant préalablement à la fécondation le nombre des chromosomes à $n$ dans chacun des gamètes, assure automatiquement, dans l'œuf résultant de l'addition de ces gamètes et par suite dans les tissus de l'em. bryon, la constance du nombre $2 n$ à travers les générations. Cela fournit à notre esprit une justification de la méiose et de sa symétrie constatée dans l'histoire des deux catégories de gamètes.

§4. - Fécondation, chromosomes et hẻrédité. Il est naturel que l'ensemble des faits précédents méiose précédant la fusion des gamètes et addition des chromosomes dans la fécondation elle-mème aient fortement impressionné les biologistes. Ils ont exercé une influence véritablement tyrannique depuis vingt ans. On a voulu en déduire l'explication complète de l'hérédité. De la symétrie que montrent les noyaux des gamètes dans la fécondation, opposée à l'inégalité qu'offrent le spermatozoïde et l'ovule quant au cytoplasme, on a conclu que ce dernier n'avait qu'une importance tout à fait secondaire et que le noyau seul était le siège des propriétés héréditaires. La fusion des noyaux est ainsi devenue, aux yeux des chercheurs, le phénomène capital, le seul même essentiel dans la fécondation.

On a été plus loin. Dans les noyaux, les chromosomes, de par la symétrie qu'ils montrent eux-mêmes, ont été regardés, à leur tour, comme les seules parties méritant considération et on a localisé en eux le substratum de l'hérédité. La chromatine est devenue la substance héréditaire spécifique.

Enfin, la constance du nombre de chromosomes, leur réapparition régulière aux divisions successives, les différences d'aspect que certains d'entre 
eux offrent d'une manière plus ou moins constante dans divers types, ont conduit à une série d'hypothèses. La plus importante consiste à regarder chacun des chromosomes comme possédant une individualité véritable et permanente (malgré que ces éléments disparaissent totalement dans l'intervalle de deux divisions cellulaires consécutives). On a imaginé, en outre, qu'ils étaient qualitativement différents les uns des autres et que chacun était le support d'une partie des prouriétés héréditaires de l'espèce. Ce sont là des notions qui jouent un rôle prépondérant dans la biologie contemporaine. Je les signale ici, parce que nous verrons plus loin les applications qu'on en a faites à certains problèmes de la Sexualité, tels que le déterminisme du sexe en particulier.

Arec une minorité de biologistes, je me refuse, pour ma part, à reconnaître à l'ensemble de ces dernières conceptions la solidité qu'on leur accorde généralement.

Ce sont une série d'hypothèses s'appuyant les unes sur les autres, mais à la base desquelles manque une preuve décisive et qui sont inspirées par une con ception matérielle des plus discutables des phénomènes de l'hérédité.

La chromatine n'a une valeur spéciale que de par notre technique; grâce à ses réactions vis-à-ris des matières colorantes, nous pouvons la distinguer. Estce véritablement une raison pour qu'elle ait dans la cellule une dignité supérieure et pour que ce qui ne se colore pas soit négligeable?

Une discussion véritable des idées formulées dans ce domaine exigerait une place considérable et l'examen détaillé de nombreux faits d'ordre histologique. Elle ne peut être entreprise ici.

Il m'a semblé nécessaire par contre de résumer brièvement les points fondamentaux de la formation 
et de la stucture des gamètes et de rappeler sommairement les traits essentiels de leur rencontre dans la fécondation, parce qu'ils constituent les éléments primordiaux de la sexualité et parce que, dans la suite, nous devrons, à maintes reprises, faire appel aux faits ou aux idées qui précèdent. 



\section{Les problèmes de la Sexualité}

\section{PREMIÈRE PARTIE}

\section{LHERMAPHRODISME OU RÉUNION DES SEXES}

Les gamètes sont produits dans l'organisme, uniquement par les glandes génitales. Mais deux possibilités se présentent : ou bien, dans un -même individu, ces glandes donneront à la fois des ovules et des spermatozoïdes, ou bien elles ne formeront que les uns à l'exclusion des autres. Dans le premier cas, les deux sexes sont réunis et l'organisme est dit hermaphrodite 1 ou encore monoique ${ }^{2}$; dans l'autre, out les sexes sont séparés, il est dit gonochorique ${ }^{3}$ ou dioique. Nous allons d'abord examiner l'hermaphro disme.

1. Hermaphrodite, personnage mythologique, fls d'Hermès et d'Aphrodite: dans l'art grec, statues couchées, où se trouvent combinées les formes de l'homme et de la femme.

2. Monoïque, de v.óvos, seul, et o:\%os, maison.

3. Yóvos. génération, yì̀p: qưós, séparation. 


\section{CHAPITRE IV}

\section{LES FORMES DIVERSES DE L'HERMAPHRODISME}

Autofécondation et fécondation croisée. - L'autofécondation chez les plantes et chez les animaux. - Lignées pures de W. Johannsen. - Amphimixie.

Les formes diverses de 1 hermaphrodisme chez les animaux: structures diverses des glandes grénitales. - Hermaphrodisme simultané et hermaphrodisme successif (protandrie et protogynie). - Mâles complémentaires

Pour cette étude, ì nous faut quitter les anımaux supérieurs, où l'hermaphrodisme n'existe que d'une manière exceptionnelle et rudimentaire, confinant à la tératologie. Au contraire, dans de vastes groupes d'animaux inférieurs et chez la généralité des végétaux, c'est une disposition normale. D'une condition à l'autre, il y a, comme nous le verrons, tous les passages.

Parmi les végétaux, la séparation des sexes est l'exception. En effet, chez les Phanérogames, les fleurs offrent, dans la règle, des étamines où se forment les gamètes mâles et un pistil, siège des gamètes femelles. Chez les Cryptogames vasculaires et chez les Mousses, où la rie de la plante, - son cycle érolutif comme on peut dire, - comprend deux générations alternant régulièrement et dont l'une n'offre pas de différenciation sexuelle ${ }^{1}$, la généra-

1. Voir infra, chap. XXI, p. 271 
tion sexuée présente, dans la majorité des groupes, les organes des deux sexes sur un même appareil végétatif (prothalle ou tige feuillée). Il y a cependant, chez ces Cryptogames, des espèces ou même des groupes, dans lesquels les sexes sont régulièrement séparés. Telles sont les Prêles, dont les prothalles sont, les uns màles, les autres femelles, pour ne citer qu'un exemple:

L'hermaphrodisme n'est pas une condition uniforme. Il y a lieu d'y établir bien des catégories. Une distinction importante est d'ordre physiologique; c'est celle de l'autofécondation et de la fécondation croisée.

\$1. - Autofécondation. - Dans l'au tofécondation, les ovules sont normalement fécondés par des spermatozoïdes provenant du même individu; chaque individu se féconde lui-même; dans la fécondation croisée, les spermatozoïdes fécondateurs proviennent, au contraire, d'un autre individu; la reproduction nécessite donc le concours de deux individus, comme si les sexes étaient séparés au point de vue morphologique.

L'autofécondation exige que les deux catégories de gamètes soient mûrs simultanément ou que, tout au moins, une disposition spéciale permette aux spermatozoïdes d'ètre mis en réserve et de subsister jusqu'à ce que les ovules soient fécondables. Il faut, en outre, que mécaniquement les premiers puissent atteindre les seconds.

L'absence d'une de ces deux conditions entraine, pour beaucoup d'hermaphrodites, l'exclusion de toute autofécondation. Mème chez les plantes où, dans la fleur, les organes des deux sexes sont si voisins, elle est souvent rendue impossible, ou du moins très difficile. Darwin avait rapprochẻ cette constatation de l'in- 
fluence fâcheuse sur la vigueur de la descendance, qu'on attribue très généralement, au moins pour l'espèce humaine, à la consanguinité des parents, et il pensait que la sélection naturelle avait tendu à faire prédominer les dispositions anatomiques ou physiologiques exclusives de l'autofécondation.

Pendant longtemps, sous la suggestion de cette idée théorique, on a cru que l'autofécondation ne jouait qu'un ròle extrêmement restreint; mais actuellement, une réaction se dessine contre cette opinion a priori. Il est d'ailleurs loin d'être démontré que la consanguinité ait, par elle-même, une action nocive sur la reproduction des organismes. Déjà, pour les animaux domestiques, l'éleveur ne s'en soucie guère, dans un grand nombre de cas. Mais. dans la nature, chez les plantes, l'autofécondation est la règle chez d'assez nombreuses espèces; c'est ce qui arrive, par exemple, quand les étamines mûrissent avant l'ouverture de la fleur et pollinisent le stigmate dans le bouton floral encore clos. Tel est le cas chez la plupart des Papilionacées, comme le pois ou le haricot. C'est celui aussi de céréales comme l'orge ou le blé. Normalement, ces diverses plantes ne se reproduisent que par autofécondation. La fécondation croisée exige, chez elles, une intervention extérieure, accidentelle ou expérimentale. L'accident est réalisé, parfois, dans le Pois, par exemple, grâce à l'intrusion d'un insecte Coléoptère (Bruchus pisi), qui perfore des boutons floraux où les étamines ne sont p̧as encore mûres et $y$ introduit ainsi du pollen provenant d'une autre fleur.

Chez les animaux, l'autofécondation normale n'a pas encore été constatée d'une façon authentique dans un grand nombre d'espèces; le fait est, d'alleurs, plus difficile à établir avec précision. Hais elle est cependant certaine chez plusieurs types et vraisemblable 
dans beaucoup d'autres. J'en énumérerai quelques exemples à titre documentaire.

Elle a lieu entre deux anneaux d'àges différents d'un mème Tænia; il semble qu'elle se produise chez. un certain nombre de Trématodes. On est obligé de l'admettre chez les Crustacés Rhizocéphales (Sacculines et Peltogasters parasites des Crabes et des Bernards-l'Ermite). Elle a été constatée chez certains Crustacés Cirripèdes (Pollicipes). Parmi les animaux coloniaux hermaphrodites (Bryozoaires, Tuniciers), si certains ne mûrissent pas simultanément leur produits génitaux des deux sexes, chez d'autres, l'autofécondation ne paraît pas pouroir être écartée. Il en est ainsi des Ascidies et spécialement des Ascidies composées. Cependant, des expériences de Castle tendraient à prouver que chez une Ascidie (Ciona intestinalis), les ovules possèdent une sorte d'immunité vis-à-vis des spermatozoïdes provenant du même individu. Par contre, l'existence de l'autofécondation a été établie d'une façon précise chez différents Vers Nématodes, par les observations si soignées de Maupas.

§ 2. - Lignées pures. - Remarquons, en passant, que l'autofécondation placeles animaux ou les plantes, qui la présentent normalement, dans des conditions très particulières au point de vue de l'hérédité. D'une génération à l'autre, c'est toujours une même suuche qui se continue sans apport d'élément étranger. En partant d'un individu donné, au bout d'un certain nombre de générations, tous les individus obtenus sont absolument comparables au point de vue héréditaire. Ils ont tous rigoureusement la même substance ancestrale. Ils forment ce que Johannsen appelle une lignée pure. Dans des organismes, hermaphrodites ou non, mais où la fécondation est croisée, il y a, au contraire, 
à chaque génération, mélange de deux lignées ancestrales infiniment complexes. Le problème de l'hérédité est beaucoup plus simple à étudier expérimentalement dans le premier cas et c'est avec raison que Johannsen a proposé de s'y attacher tout d'abord.

\$3. - Amphimixie. - En considérant, arec Darwin, la fécondation croisée comme le processus général et normal, seul capable d'assurer la vigueur des races, on en était renu à voir dans le mélange, à chaque génération, des substances héréditaires,-_dans l'amphimixie, suivant le terme créé par Weismann, - le caractère essentiel, la raison d'être primordiale, en quelque sorte, de la fécondation. On arait identifié les notions d'amphimixie et de fécondation et nous verrons la réaction qui s'est produite contre cette interprétation ${ }^{1}$.

\section{$\$ 4$. - Formes diverses de l'hermaphrodisme chez} les animaux. - Anatomiquement, l'hermaphrodisme offre, chez les animaux, une très grande variété de dispositions, que je n'ai pas l'intention d'examiner ici en détail ; mais il est intéressant d'en signaler rapi-dement les principales.

Considérons d'abord des types où l'hermaphrodisme est normal et simultané. Les ovules et les spermatozoïdes se forment, chez certains animaux, còte à côte, non seulement dans la même glande, dite glande hermaphrodite, mais dans les mêmes culs-de-sac terminaux de celle-ci. C'est ce qui arrive, par exemple, chez les Escargots. Et cependant, chez eux, il n'y a pas autofécondation. Produites au contact les unes des autres, les deux catégories de gamètes, une fois mùrs, suivent des canaux distincts et sont dès lors définitivement et complètement séparés.

1. V. p: 234 . 
Ailleurs, on trouve deux glandes distinctes, ayant chacune des conduits efférents propres : l'une est un testicule et l'autre un ovaire; pour fixer les idées, on observera cette disposition chez les Vers de terre (Lombrics), les Sangsues, les Douves (Trématodes) et les Tænias (Cestodes).

Entre les deux cas précédents, il serait facile de trouver toutes les transitions. Dans la glande hermaphrodite de certains Mollusques, une partie des culsde-sac sont mâles et d'autres, voisins, sont femelles. Ou bien toute une région de la glande est d'un sexe, le reste étant du sexe opposé. G'est ce que montrent, par exemple, les coquilles Saint-Jacques (Pecten maximus). A l'époque de la reproduction, la glande génitale forme une très volumineuse masse en saillie (appelée vulgairement bosse de Polichinelle), dont la portion distale rouge est un ovaire et la portion proximale blanche est un testicule. Sans sortir du groupe des Mollusques, on trouverait toutes les dispositions possibles, et des glandes proprement dites, et de leurs conduits évacuateurs.

$\S 5$. - Hermaphrodisme simultané et hermaphrodisme successif. - La production des ovules et des spermatozoïdes considérée dans le temps ne donne pas lieu à une moindre variété.

D'assez nombreux animaux sont hermaphrodites simultanés, les deux catégories de gamètes y sont mûrs en même temps. Tels sont les Escargots, pour nous borner à un des exemples précédemment cités. De là à l'hermaphrodisme successif proprement dit, où l'animal a d'abord des testicules, puis, plus tard, des ovaires, après régression complète des testicules. il y a tous les passages.

Tout d'abord, chez des hermaphrodites simultanés, l'une des deux glandes, le testicule le plus souvent, 
peut arriver à maturité un peu avant l'autre; c'est le cas chez beaucoup d'espèces du groupe des Tuniciers, en particulier chez les Ascidies composées; mais pendant la plus grande partie de la période de reproduction, ovules et spermatozoïdes sont émis cóte à côte.

L'écart entre les époques de maturité des uns et des autres s'accentuant, on arrive à des cas où elles ne coïncident plus du tout. L'hermaphrodisme est alors devenu successif; on l'appellera protandrique quand c'est le testicule qui mûrit le premier, et protogynique quand, au contraire, c'est l'ovaire. Ce second cas est beaucoup plus rare que le premier. On le connait chez quelques espèces isolées parmi les Turbellariés (Prostomum lineare), chez des Limaces et chez les Salpes parmi les Tuniciers.

La protandrie est donc le cas le plus général. Les Vers solitaires ou Tænias en offrent un exemple typique. Leur corps est un long ruban divisé en anneaux, parfois au nombre de plusieurs centaines, dans chacun desquels se développe un système génital complet, testicules, ovaires et glandes annexes. Mais les anneaux jeunes sont uniquement mâles, alors que, dans les plus âgés, ou proglottis, les testicules ont disparu tandis que les ovaires se sont développés. Le même anneau est successirement mâle et femelle, et les anneaux jeunes s'appliquant contre les proglottis, les fécondent.

L'hermaphrodisme successif est aisé à reconnaitre quand l'une des glandes n'a pas totalement disparu au moment où l'autre s'ébauche. S'il en est autrement et si l'on ne peut pas suivre le mème individu à ses phases successives, on se croira en présence de mâles ou de femelles distincts les uns des autres. Plusieurs cas d'hermaphrodisme normal ont été ainsi longtemps méconnus ou contestés ou le sont même encore 
Il en a été ainsi, par exemple, pour une famille de Crustacés Isopodes, les Cymothoadiens, qui vivent en parasites sur la peau de Poissons marins (fig. 16 A). Or. sait maintenant qu'ils sont màles à l'état jeune, puis deviennent femelles; on a vu les diverses étapes de la substitution d'ovaires aux testicules, et en même
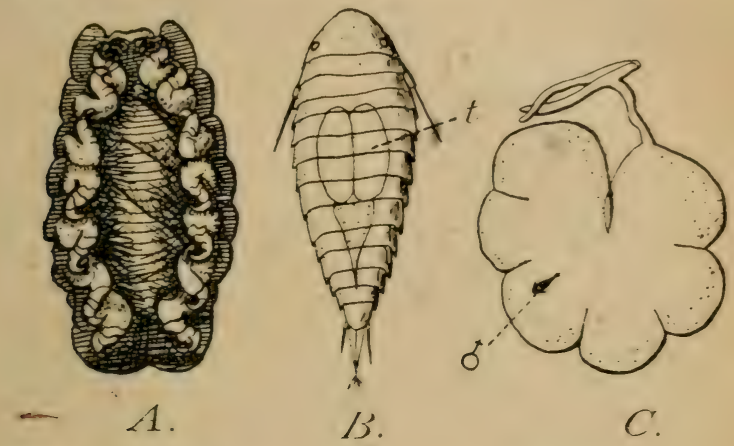

Fig. 16. - Exemples d'Isopodes offrant l'hermaphrodisme successif protandrique. $A$, un Cymothoa vu ventralement (d'après Boas); $B$, un Danalia (Cryptoniscien), à l'état mûle, fortement grossi, $t$, testicules; $C$, stade femelle ultérieur du méme individu. Noter sa métamorphose. La tache noire ơ représente mále se déplaçan sur la femelle et donne une idée sur la différence de taille ence les deux états successirs.

temps les transformations des orifices sexuels corrélatifs.

C'est ce que montrent aussi, dans des conditions qui étaient encore beaucoup plus difficiles à mettre en évidence, les Cryptonisciens, autre famille de Crustacés Isopodes, également parasites, mais appartenant aux Epicarides qui vivent aux dépens d'autres Crustacés et non de Poissons. Tous ces animaux sont déformés par le parasitisme à un degré extrême, et constituent, à cet égard, une série d'exemples parmi les plus beaux au point de vue de la théorie de l'Evo- 
lution. D'une manière générale, - nous aurons l'occasion de d'y revenir plus loin, - les sexes sont séparés chez les Epicarides; les mâles restent nains et sont relativement peu déformés (fig. 16), tandis que les femelles deviennent proportionnellement énormes; elles sont rendues méconnaissables par les métamorphoses qu'elles subissent et dont les figures 16 et 18 donnent une idée. Les apparences sont les mêmes chez les Cryptonisciens que chez les autres Epicarides, et cependant la réalité est autre. Tous les individus jeunes sont mâles. Ils ont encore la forme larvaire, vivent librement et n'ont subi aucune déformation du type Isopode. Après avoir fonctionné un certain temps comme mâles, ils vont se fixer sur l'espèce de Crustacé qui leur sert d'hôte, y perdent leurs appendices locomoteurs, grossissent beaucoup en changeant complètement d'aspect extérieur, au point qu'on ne soupconnerait plus en eux des Crustacés, à les voir à cet état (roy. fig. $16 \mathrm{C}$ ). Intérieurement, pendant ce temps, leurs testicules se sont atrophiés et il s'est développé graduellement des ovaires et des oviductes. Le mâle s'est transformé en une femelle et cela-est la règle universelle dans toute cette famille des Cryptonisciens.

Je mentionnerai encore un exemple analogue et non moins significatif, emprunté à un autre groupe d'animaux marins, les Myzostomes, type roisin des Annélides et parasite sur des animaux d'un autre groupe bien défini, les Crinoïdes ou Lis de mér, qui appartiennent à l'embranchement des Échinodermes. Leur parasitisme offre des degrés différents. Certaines espèces sont parasites extérieurs et se déplacent sur le disque et les bras des Crinoïdes; d'autres sont des parasites plus stricts, fixés à demeure en un point de l'hôte. Les premières (telles que l'espèce Myzostoma cirriferum) sont nettement hermaphrodites; les tes- 
ticules sont mûrs chez les jeunes et l'ovaire, déjà présent mais rudimentaire, se développe ensuite. Chez les secondes (ex.: $\boldsymbol{M}$. pulvinar), on trouve de grosses femelles fixées à l'hòte et, sur celles-ci, de minuscules mâles mobiles. On a donc cru d'abord que ces dernières espèces avaient les sexes séparés et que le màle était nain. Mais des exemplaires de tailles farorables ont montré que c'était le mâle lui-même qui devenait ensuite femelle : il y a encore ici hermaphrodisme successif, mais le testicule a presque complètement disparu, quand on commence à distinguer l'ébauche de l'ovaire, et comme on ne peut pas suivre le même individu durant toute son existence, la phase où on peut effectivement constater l'hermaphrodisme est très fugitive.

J'ai insisté sur ces quelques exemples parce qu'ils montrent la variété des dispositions qu'offre la nature, et la difficulté qu'il y a à résoudre dans chaque cas particulier des problèmes en apparence très simples.

§6. - Mâles complémentaires. - Il y a enfin des cas que je me borne à signaler et où les conditions de sexualité de l'espèce sont pius complexes encore. Les individus normaux, en quelque sorte, sont de parfaits hermaphrodites; mais il existe, en outre, des mâles nains, rudimentaires. C'est ce que montrent certains Crustacés fixés de l'ordre des Cirripèdes, roisins des Anatifes (Alcippe, Scalpellum). Darwin, qui a découvert le fait, a donné à ces individus le nom de mâles complémentaires. On en a trouvé depuis dans d'autres groupes d'Invertébrés. 


\section{CHAPITRE V}

\section{L'EXTENSION ET LES DEGRÉS DE L'HERMAPHRODISME CHEZ LES ANIMAUX}

IIermaphrodisme normal; ses divers degrẻs de généralité. Hermaphrodisme exceptionnel. - Recherches d'E. Maupas sur les Nématodes. - Hermaphrodisme rudimentaire; cas divers chez les Vertébrés (Myxine et autres Poissons; organe de Bidder des Crapauds, etc.). - Hermaphrodisme accidentel des Mammifères et de l'Homme.

L'hermaphrodisme est-il une condition primitive ou secondaire des organismes?

Si nous envisageons maintenant la répartition de l'hermaphrodisme dans le règne animal, nous constatons d'abord qu'il est très répandu, mais arec des degrés de généralité très différents suivant les groupes.

$\S 1$ - Hermaphrodisme normal. - Ses diver's degrés de généralité. - Il y a des classes d'animaux entièrement hermaphrodites. Tels sont les Turbellariés, les Trématodes et les Cestodes, c'est-à-dire tout l'embranchement des Plathelminthes, moins les Némertiens; parmi les Vers, les ordres des Oligochètes (ex. : Lombric) et les Hirudinées (Sangsues); tels sont encore les ordres des Bryozoaires et des Tuniciers. 
Dans la classe des Crustacés, l'ordre des Cirripèdes est presque entièrementformé d'hermaphrodites. Dans l'ordre des Isopodes, les familles des Cymothoadiens et des Cryptonisciens le sont également. De mème chez les Échinodermes, dans la classe des Holothuries, l'ordre des Synaptes.

D'autres fois, dans un ordre où les sexes sont généralement séparés, certains genres, du, dans un genre, certaines espèces les ont réunis. L'hermaphrodisme, dans ces cas, dont on pourrait citer toute une gradation, tend à prendre l'allure d'un phénomène exceptionnel.

Il est plus nettement exceptionnel quand, dans un groupe à sexes séparés, une seule espèce présente, d'une façon plus ou moins constante, de l'hermaphrodisme; on le constatera sur un certain pourcentage d'individus, ou encore dans certaines localités et pas dans d'autres. Cet hermaphrodisme pourra être fonctionnel ou plus ou moins imparfait; les organes d'un sexe seront alors bien développés, les.autres étant à un état plus ou moins rudimentaire; ou bien, chez les individus hermaphrodites, aucun des deux sexes ne sera bien conformé. On conçoit, par cette simple énumération, la variété des aspects que peut revêtir l'hermaphrodisme.

Pour fixer les idées, je citerai quelques exemples. Les Crustacés Décapodes (ex. : Homards, Ecrevisses, Crevettes, Crabes) constituent essentiellement un grqupe d'animaux gonochoriques. On connait cependant actuellement quelques rares espèces de cet ordre, qui montrent un certain degré d'hermaphrodisme. Ainsi, chez une Gébie du Japon (Gebia major), on observe des femelles pures et des mâles offrant, en arrière des testicules, des ovaires plus ou moins développés. Une jolie crevette bleue de notre littoral, Lysmata seticaudata, offre régulièrement à la fois des 
ovaires et des testicules, dés oviductes et des canaux déférents. Chez les Vers 'de' l'ordre des Annélides Polychètes, oủ les sexes sont normalement séparés, on trouve des genres et des espèces hermaphrodites et aussi, dans d'assez nombreuses espèces gonochoriques, des individus exceptionnellement hermaphrodites à des degrés divers.

Les Etoiles de mer sont gonochoriques. Or, une petite espèce de nos côtes, Asterina gibbosa, s'est montrée régulièrement hermaphrodite, quoique à des degrés variés, dans diverses localités où on l'a observée (Roscoff, Banyuls), alors qu'à Naples elle est représentée par des individus unisexués et par quelques rares hermaphrodites.

L'étude précise de l'hermaphrodisme, dans les groupes où il s'est limité à quelques espèces exceptionnelles, conduit souvent à des constatations très intéressantes au point de rue général. C'est ce dont témoignent, par exemple, les résultats acquis sur les Nématodes, Vers dont l'immense majorité est gonochorique. Une espèce parasite dans le poumon des Grenouilles (Rhabdonema nigrovenosum) est hermaphrodite. Or, on a reconnu que ses ceufs donnent naissance à une génération où les sexes sont séparés et qui vit dans la terre; mâles et femelles de cétte génération s'accouplent, produisant à leur tour la génération qui parasite la Grenouille et qui est hermaphrodite. Voilà donc une espèce animale où alternent régulièrement une génération hermaphrodite parasite et une génération gonochorique libre.

Recherches d'E. Maupas sur les Nématodes. - Les belles recherches de Maupas ont fait connaitre, parmi les petits Nématodes libres vivant dans la terre, diverses espèces hermaphrodites dont cet auteur a fait des. 
cultures pures. Il a constaté que, chez elles, l'hermaphrodisme était constant. Il en a obtenu une série de générations dont les individus s'autofécondaient. Parmi des milliers d'individus hermaphrodites, apparaissait de temps à autre un mâle. Ainsi, chez Rhabditis elegans, dont Maupas a suivi 50 générations consécutives, sur 20.032 individus étudiés, 30 seulement étaient des mâles; ils auraient évidemment passé inaperçus sans le soin apporté aux observations. Chez ces Nématodes, les hermaphrodites sont, en réalité, des femelles, dont la glande génitale a acquis la propriété de produire par surcroit des spermatozoïdes; corrélatirement, les mâles ont peu à peu disparu, ou sont en voie de disparaître. L'hermaphrodisme résulte ici nettement d'une transformation secondaire du sexe femelle. Dans les conditions naturelles, l'autofécondation " est devenue la règle absolue.

Mais Maupas a réussi, à l'aide des quelques mâles qu'il avait obtenus, à faire des fécondations croisées et à cultiver des individus qui en étaient issus. Or, chez Rhabditis elegans, les œufs issus de fécondation croisée lui ont donné 463 mâles pour 1.000 femelles. La fécondation croisée, dans cette espèce, quand on réussit à l'obtenir, fait donc réapparaitre le sexe mâle dans une forte proportion (près de $50 \%$ ). Mais cette , conclusion, valable pour Rhabditis elegans, ne saurait être généralisée, comme l'ont montré à Maupas des expériences parallèles sur des espèces hermaphrodites très voisines, telles que $R h$. marionis, et je relève ces faits pour montrer combien ces phénomènes sont complexes et combien il faut être prudent dans les généralisations.

Maupas a pu faire d'ailleurs, sur ces mèmes animaux, d'autres constatations non moins intéressantes. Chez plusieurs espèces, en effet, les rares màles qu'il 
obtenait ${ }^{1}$, quoique normalêment constitués, ne s'accouplaient pas avec les femelles : il semble bien que dans ces formes devenues normalement hermaphrodites, le mâle, quand il réapparait exceptionnellement, a perdu l'instinct sexuel.

On voit donc, par ces quelques exemples, que les variations de la sexualité chez les Nématodes ne sont pas des phénomènes simples et uniformes.

§2. - L'hermaphrodisme chez les Vertébrés. Chez les Vertébrés, qui sont essentiellement gonochoriques, on connaît cependant un certain nombre de faits d'hermaphrodisme plus ou moins complet, sur lesquels il convient de s'arrêter un peu.

La sexualité de la Myxine. - L'un d'eux, le plus connu peut-être, a été signalé en 1887, simultanément par Cunningham et par Fritjof Nansen, le futur explorateur polaire, chez la Myxine. Cette espèce appartient au groupe le plus inférieur des Poissons, les Cyclostomes (dont font aussi partie les Lamproies). On la pêche, en particutier, sur les côtes de Norvège et on la trouve souvent fixée sur les Morues dont elle perfore même la paŕoi abdominale; on l'a considérée, pour cette raison, comme un parasite, mais c'est, en réalité, un carnassier s'attaquant notamment aux poissons éjà pris à la ligne. Cunningham et Nansen annon cèrent que la Myxine est, d'une façon normale, hermaphrodite protandrique : mâle fonctionnel dans le jeune âge, femelle fonctionnelle ensuite. Les glandes génitales, chez le jeune, ont la structure de testicules

1. Voici quelques chiffres à titre de document:

Rhabditis guignardi. Sur 12.657 individus, trouvé 2 mâles.

Rh. dolichura.... - 7.136

Rh. viguieri. . . . - 1.000

Diplogaster robustus. - 23.445

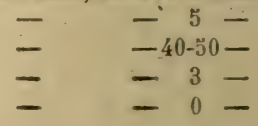


dans leur portion antérieure et d'ovaires dans leur portion postérieure. Les testicules s'atrophieraient après aroir fonctionné. Les recherches récentes de M. et $M^{\text {mo }}$ Schreiner ont rerisé cette conclusion : l'hermaphrodisme est réel en tant que structure histologique de la'glande, mais il n'est pas fonctionnel. Après aroir présenté les ébauches des deux sexes, en effet, chaque individu perd l'un ou l'autre par atrophie, mais ne fonctionne soit que comme màle, soit que comme femelle; certains individus resteraient même stériles, aucune des deux ébauches ne triomphant de l'autre et ne s'achevant.

L'organe de Bidder des Crapauds mâles. - Un autre type de Vertébrés montre encore, d'une façon régulière, un hermaphrodisme imparfait fort intéressant: c'est le Crapaud mâle. En avant de chaque testicule, se trouve, en effet, un organe (fig. 17) occupant la situation correspondant à la partie antérieure de l'ovaire des femelles et qui a reçu le nom d'organe de Bidder. Sur un mâle jeune, on constate que cet organe renferme des ovules; mais ceux-ci restent de petite taille et dégénèrent, tandis qu'il s'en forme constamment,de nouveaux qui ont le mème sort. Aucun n'arrive à maturité. Les organes de Bidder, chez les mâles des Crapauds, sont donc des ovaires rudimentaires, qui ne jouent plus le rôle de glande

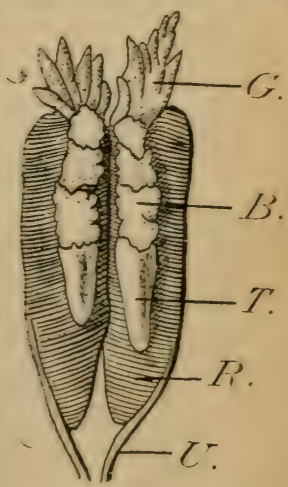

Fic. 17.-Reins et organes génitaux diun crapaud mảle (Bufo variabilis) (daprès hinappe:

$R$, rein; $L$, uretere; $T$, teз ticule; $G$, corps graisseux; $B$, organe de Bidder entre .e testicule et le corps graisseux). génitale, et qui cependant ont une fonction essen- 
tielle, car leur ablation totale entraine rapidement. la mort. Cette fonction n'est pas connue actuellement d'une façon précise, mais il est très vraisemblable qu'elle consiste à rejeter dans le sang une substance provenant de la transformation des ovules au moment où ils disparaissent. L'organe de Bidder est le premier exemple que nous rencontrons d'une glande à sécrétion interne en rapport avec les glandes génitales et nous verrons plus loin ${ }^{1}$ le rôle capital des. organes de cette nature dans les phénomènes de la. sexualité.

On peut encore citer d'autres phénomènes se rattachant à l'hermaphrodisme chez les Vertébrés, mais moins réguliers que les précédents et montrant toute une gradation, jusqu'à se réduire à des anomalies. indiriduelles des plus rares et des plus rudimentaires.

L'hermaphrodisme chez les Poissons osseux. - On connait divers Poissons osseux (Téléostéens), où un hermaphrodisme plus ou moins accentué est, soit constant, soit très fréquent. Plusieurs espèces de Serrans, que l'on pêche communément en Méditerranée, ont des glandes génitales constamment hermaphrodites et mûriraient à la fois des spermatozoïdes et des ovules. Aristote avait déjà signalé ce fait. La Daurade (Chrysophrys aurata) a, presque toujours aussi des glandes histologiquement hermaphrodites. Le Bogue (Box salpa), les Pageaux (Pagellus mormyrus), les Sars (Sargus annulatus) offrent une proportion plus ou moins élevée d'individus à glandes mixtes, mais sans que, dans ces divers cas, il $y$ ait hermaphrodisme fonctionnel. Beaucoup d'autres espèces montrent, avec une rareté plus ou moins.

1. Ch. X, p. 11 . 
grande, des rudiments du mème ordre; tels sont les Harengs, les Morues, les Maquereaux, les Merlans, les Perches; ces direrses espèces étant comestibles, il en a été manié un nombre énorme d'individus, ce qui a permis de constater ces dispositions exceptionnelles. Des faits analogues peurent donc ètre plus répandus qu'on ne le suppose; mais il ne s'agit jamais que d'ébauches histologiques plus ou moins rudimentaires.

L'hermaphrodisme rudimentaire' chez les Mammifères et l'Homme. - Un hermaphrodisme rudimentaire se rencontre aussi à des degrés divers, et arec une rareté plus ou moins grande, chez les Mammifères et, en particulier, chez l'Homme. C'est mème chez l'Homme qu'on en connait le plus de cas, par la simple raison que c'est l'espèce dont tous les individus sont le plus complètement observés.

L'hermaphrodisme de l'Homme et des Mammifères se révèle généralement, sur un individu, par un mélange des caractères extérieurs des deux sexes. orifices et organes génitaux externes ou formes générales du corps; c'est ce que l'art antique a exprimé sans connaitre les glandes génitales elles-mêmes. Ces malformations s'accompagnent parfois de troubles des instincts sexuels. Tous les degrés existent, depuis - une légère déviation par rapport à l'un des sexes. jusqu'à un mélange plus ou moîns complet des caractères morphologiques et des fonctions, rendant parfois difficile l'attribution à l'individu d'un sexe donné 1 .

En fait, cependant, les malformations extérieures. de ce genre ne sont pas toujours accompagnées d'un

1. Les faits relatifs à l'hermaphrodisme chez l'homme ont été. l'objet récemment d'une grande monographie de Neugebaur (Hermaphroditismus.beim Menschen, Leipzig, 1908). 
état hermaphrodite de la glande, et l'on a distingué, suivant que celui-ci est ou non réalisé, l'hermaphro. disme vrai et le faux hermaphrodisme. Mais, même dans le premier de ces cas, il ne s'agit le plus souvent que d'une altération très partielle des glandes génitales, et non d'un hermaphrodisme vraiment fonctionnel. Aucun de ces individus hermaphrodites ne peut remplir complètement, à la fois le rỏle de mâle et celui de femelle. Ces cas individuels sont d'un haut intérêt en ce qu'ils mettent en évidence les rapports entre la glande génitale et les conduits génitaux ou les caractères sexuels secondaires ${ }^{1}$. Ils rentrent, au point de vue morphologique, dans la catégorie de faits que l'on a désignée sous le nom de gynandromorphisme 2 , sur laquelle nous revenons plus loin.

D'autre part, ils nous montrent que si, chez les types gonochoriques, la glande génitale évolue normalement, d'une facon totale, vers l'état, soit de testicule, soit d'ovaire, il n'y a pas cependant une incompatibilité absolue entre la différenciation des deux catégories de gamètes. Ce sont probablement des facteurs d'ordre quantitatif qui entraînent au début du développement, l'organisme vers un pôle ou vers l'autre. Aussi l'hermaphrodisme rudimentaire se rencontrerait-il vraisemblablement chez toutes les espèces, si on le recherchait dans un nombre suffisant d'individus.

§ 3. - Lhermaphrodisme est-il une condition primitive ou secondaire des organismes. - En étudiant la répartition de l'hermaphrodisme dans le règne animal, nous avons constaté qu'il se présentait dans des conditions variées : ici, anomalie individuelle et rudiment; là, phénomène normal, mais limité à des

1. Voir à ce sujet le chapitre IX.

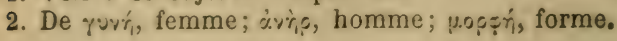


espèces ou à des genres particuliers; ailleurs enfin, trait fondamental d'organisation dans des groupes étendus.

$\mathrm{Si}$, conformément aux idées évolutionnistes, on ne considère plus la dirersité des faits que nous présentent les organismes comme l'expression des caprices d'un Créateur, on ne peut manquer de se demander quel est le déterminisme de l'hermaphrodisme et si c'est un état qui a succédé au gonochorisme ou inversement. Cela a été le sujet de longues discussions, auxquelles ont pris part un grand nombre de naturalistes éminents du dernier demi-siècle; ils ont conclu de façons très discordantes, preuve que la solution du problème est malaisée, ou qu'il n'a pas été compris de même par tous.

La diversité mème des conditions dans lesquelles se montre l'hermaphrodisme impose de distinguer ici divers cas.

Il est évident que l'hermaphrodisme accidentel et plus ou moins rudimentaire, tel que nous l'arons envisagé en dernier lieu, est un phénomène secondaire. On peut en dire autant de l'hermaphrodisme normal que présentent des espèces isolées, dans des groupes où le gonochorisme est la règle générale. Si nous nous reportons aux exemplés qui précèdent, nous conclurons ainsi pour les Crustacés Décapodes, tels que Gebia major ou Lysmata seticaudata, pour les Nématodes étudiés par Maupas, et pour les divers Poissons que nous arons cités. On peut même dire, avec assez de certitude, pour la plupart de ces cas, que l'hermaphrodisme s'est greffé sur le sexe femelle 1 .

L'hermaphrodisme semble bien aussi être un état

1. Inversement d'ailleurs, dans des groupes entièrement hermaphrodites, comme les Trématodes, on connait de rares espèces - ex. : Bilharzia hæmatobia, parasite du sang de 
secondaire, quand nous le constatons chez des ensembles d'animaux plus considérables, mais se distin- guant des formes voisines par des mœurs particulières ou, comme on dịt, par une éthologie spéciale, telle que le parasitisme ou la vie sédentaire. Il en sera ainsi, par exemple, des Cymothoadiens ou des Cryptonisciens parmi les Crustacés Isopodes, de certains Mollusques Gastéropodes parasites des Echinodermes; l'hermaphrodisme y est une conséquence secondaire du parasitisme.

Doit-on aussi regarder l'hermaphrodisme comme ayant succédé au gonochorisme dans des groupes où il existe d'une façon générale, ou bien est-ce là un état primitif?

La plupart des zoologistes ont penché pour la dernière alternative et je citerai ici les noms de Haeckel, Gegenbaur, Claus, Giard, parmi ceux qui ont défendu cette idée. Ils l'appuyaient surtout sur la constatation que les types hermaphrodites sont inférieurs par leur organisation, et sur la raison a pricri que le gonochorisme est un état plus différencié, arec division du travail entre les individus; or, on admet d'une façon générale que l'évolution s'est faite de l'homogène à l'hétérogène, par un progrès constant dans la division du travail.

En réalité, ces raisons n'ont pas grande valeur, ainsi que l'a montré Fritz Müller, qui a déreloppé l'opinion inverse, c'est-à-dire l'antériorité du gonochorisme sur l'hermaphrodisme ${ }^{1}$.

lHomme - où les sexes sont séparés: ces cas très rares de sonochorisme sont évidemment des modifications secondaires de lihermaphrodisme primitif, par atrophie des parties correspondant à l'un des deux sexes.

1. Parmi les auteurs qui ont soutenu une thèse analogue, on peut citer Delage (pour les Rhizocéphales : Sacculines) et. P. Pelseneer (pour les Mollusques). 
La division du travail, ou l'hétérogénéité, peurent fort bien, dans le domaine de la sexualité, être antérieures à la formation même des groupes d'organismes pluricellulaires. Chez beaucoup d'unicellulaires, nous connaissons, en effet, des phénomènes de sexualité dont la différenciation ne le cède en rien d'essentiel à ce nous montrent les animaux supérieurs ${ }^{1}$.

Si les types hermaphrodites ont, pour la plupart, une organisation assez simple ou, si l'on veut, inféférieure, il ne s'en suit pas que ce soient des formes primitives, au contraire. Leur simplicité est plutôt de nature régressive; elle est une simplification qui parait déterminée par leur mode de vie, où les fonctions de relation sont amoindries.

La généralité des types hermaphrodites est, en effet, composée, soit d'animaux parasites, soit d'animaux vivant fixés à un support, ou tout au moins peu mobiles. Et si l'hermaphrodisme est une condition générale chez les Végétaux, ceux-ci sont tous des organismes fixés.

Si l'on compare dans un mème groupe, embranchement, classe ou ordre. les formes gonochoriques et hermaphrodites, les premières sont celles qui ont les caractères anatomiques ou éthologiques vraiment primitifs. Pour prendre un exemple, chez les Vers, les groupes hermaphrodites sont des animaux d'eau douce ou terrestres [Oligochètes (Lombrics) et Hirudinées (Sangsues)], tandis que les formes marines (Annélides Polychètes) ont, d'une façon générale, les sexes séparés. Or, les types d'eau douce ou terrestres résultent d'une adaptation secondaire des types marins originels. L'hermaphrodisme n'est donc pas, chez les Vers, le fait des formes primitives, mais bien

1. V. sixième partie, chapitres XXII-XXIV. 
des formes dérivées. Un raisonnement analogue pourrait ètre appliqué à la plupart des groupes.

Les organes génitaux des groupes hermaphrodites - glandes sexuelles et conduits évacuateurs - sont d'ailleurs anatomiquement plus compliqués que ceux des formes gonochoriques des mêmes embranchements. C'est ce qui apparaîtra nettement à toute personne familiarisée avec l'anatomie comparée des Invertébrés, quand elle mettra en parallèle l'appareil génital d'un Némertien (gonochorique) et celui d'un Turbellarié (hermaphrodite), par exemple, ou d'une Annélide Polychète et d'une Oligochète, ou d'un Gastéropode Prosobranche et d'un Opisthobranche ou d'un Pulmoné (Escargot). Pour les Mollusques, Pelseneer a même montré, par de bonnes raisons, que les canaux éracuateurs mâles, chez les types hermaphrodites, ne correspondent pas à ceux que l'on trouve chez les formes gonochoriques, mais qu'ils sont des acquisitions nouvelles et il en conclut que l'hermaphrodisme est un état secondaire qui s'est greffé sur le sexe femelle en le compliquant. La structure anatomique de l'appareil génital, telle qu'on la constate chez les types gonochoriques, ne peut donc pas résulter purement et simplement de l'atrophie des parties correspondant à l'un des sexes chez les hermaphrodites. Elle ne peut pas en être considérée comme une simple rẻduction.

Ainsi la répartition de l'hermaphrodisme dans le règne animal et l'anatomie de l'appareil génital ne fournissent pas de raisons véritables, ni surtout décisives, en faveur de l'opinion qui voit, dans la réunion des deux sexes, la condition originelle, d'où le gonochorisme dériverait par la disparition de la partie mâle ou de la partie femelle.

Il est donc plus plausible d'admettre que la séparades sexes est l'état primitif. Cependant, il ne faut pas 
oublier que nous ne connaissons, en somme, rien des phases vraiment initiales de l'Érolution, de celles au cours desquelles ont effectivement apparu les grands types du règne animal, où se sont différenciées les fonctions essentielles et où se sont établies les lignes fondamentales de l'anatomie. Pour un certain nombre des types actuels, il a pu se produire, au cours des étapes qu'ils ont parcourues, plus d'une alternance de l'état hermaphrodite et de l'état gonochorique. Nous voyons d'ailleurs actuellement, dans des cas particuliers, ainsi que nous le faisions observer plus haut, aussi bien le gonochorisme dériver de l'hermaphrodisme fcas de Bilharzia haematobia) que la transformation inverse. Cette dernière est, il est rrai, beaucoup plus fréquente. Nous pourons donc imaginer que, si pour certains groupes, l'hermaphrodisme dérive du gonochorisme, celui-ci ait pu être, à son tour, précédé d'un état hermaphrodite.

Nous ne pourons nous flatter, en d'autres termes, de connaitre les conditions vraiment primitives de la sexualité. Les considérations précédentes, comme la plupart de celles qui sont relatives à la phylogénie, ne sont jamais que des conjectures plus ou moins vraisemblables. 


\section{DEUXIÈME PARTIE}

\section{LE GONOCHORISME OU SÉPARATION DES SEXES LES CARACTĖRES SEXUELS SECONDAIRES}

L'ensemble des faits relatifs à l'hermaphrodisme indique qu'il n'est pas séparé par un fossé profond du gonochorisme; on trouve, en effet, tous les degrés dans l'extension de l'hermaphrodisme et dans sa réalisation. Si les organismes de certains groupes sont irrémédiablement et parfaitement hermaphrodites ou gonochoriques, chez d'autres, la limite distinctive de ces deux états se dérobe. Là, comme partout, les catégories que notre esprit conçoit ne répondent pas à des rẻalités générales absolument tranchées.

Ces réserres faites, il subsiste que, chez le plus grand nombre des animaux, la séparation des sexes est, en pratique, complète et fatale. Il y a, pour l'espèce, deux formes d'équilibre possibles : dans l'une, la gonade produit dess spermatozoïdes; dans l'autre, elle produit des orujes. A chacun de ces deux cas correspond une cor/stitution particulière de l'individu et de ses divers organes.

Ces deux états sont les deux sexes et on entrevoit immédiatement une question qui se pose. Est-ce la constitution générale de l'individu, au début de son existence, qui détermine l'évolution particu- 
lière de la glande génitale ou la nature de celle-ci est-elle la donnée primaire qui influe sur le reste de l'organisme?

Nous analyserons tout d'abord les éléments du dimorphisme sexuel et envisagerons ensuite les rapports entre les glandes génitales et le reste de l'organisme. Nous aurons enfin, à la lumière des faits acquis, à examiner quels sont les facteurs d'où résulte, chez les espèces gonochoriques, la détermination d'un sexe ou de l'autre dans chaque individu. 


\section{CHAPITRE VI}

\section{DIMORPHISME ET POLYMORPHISME SEXUELS}

Caractères sexuels primaires et secondaires. - Degrés divers du dimorphisme sexuel. - Cas de dimorphisme sexuel intense; corrẻlations avec l'éthologie. - Diversité des caractères sexuels secondaires.

Polymorphisme sexuel : chez certains Papillons; chez les Insectes sociaux.

Gynandromorphisme.

\$1. - Caractères sexuels primaires et secondaires. - Dans une espèce gonochorique, les deux sexes diffèrent plus ou moins nettement. Il y a un dimorphisme sexuel plus ou moins évident et accentué. Il porte sur l'ensemble de l'organisme. Nous l'analysons et le concevons sous les formes d'un certain nombre de caractères différentiels, abstractions plus ou moins arbitraires, mais indispensables:

On peut grouper ces caractères en deux et même en trois catégories :

$1^{\circ} \mathrm{La}$ nature de la glande génitale proprement dite, ovaire ou testicule. C'est le caractère fondamental que nous pouvons appeler primaire.

Les autres différences sont secondaires, mais il sera commode d'y distinguer deux groupes.

$2^{\circ}$ Une première série est étroitement corrélative de la nature des gonades; ce sont celles relatives aux organes évacuateurs des produits génitaux ou en rapport immédiat avec la fécondation et le développe_ 
ment des œufs : la femelle possédera des oviductes arec différenciation plus ou moins compliquée d'utérus, de vagin, etc.; le mâle, des canaux déférents, accompagnés ou non de glandes annexes, des vésicules séminales où s'accumule le sperme, un appareil copulateur servant à l'introduction de ce sperme dans la femelle, etc. Cet ensemble d'organes offre dans le règne animal une extrême diversité anatomique, mais que nous laissons entièrement de côté ici. Il fait partie intégrante de l'appareil génital qui, sans lui, serait incomplet et non fonctionnel. Physiologiquement, on peut dire que c'est un ensemble de caractères sexuels primaires et cependant on peut concevoir une dissociation entre lui et les gonades. En fait, d'ailleurs, dans l'embryogénie, son ébauche est indépendante de celle des glandes sexuelles et l'on constate parfois, à titre d'anomalies, chez un individu, l'association de glandes d'un sexe et de voies efférentes, partiellement ou totalement conformées comme dans le sexe opposé.

$3^{\circ}$ En dehors des organes génitaux, les deux sexes diffèrent par une série de caractères atteignant d'une façon plus ou moins visible tous les organes et qu'on oppose plus particulièrement aux deux précédents sous le nom de caractères sexuels secondaires.

La délimitation des caractères sexuels primaires et secondaires, arbitraire par sa nature, n'est pas toujours aisée dans la pratique. Il y a, en effet, en dehors du système génital, des organes qui sont liés plus ou moins directement à la fonction de reproduction. On peut se demander si les caractères correspondants doivent être placés dans la seconde ou la troisième catégorie.

\$2. - Les degrés du dimorphisme sexuel. - Prenons d'abord une idée générale du dimorphisme sexuel. On 
le conçoit plus spécialement comme l'ensemble des différences que présentent, à l'extérieur, les deux sexes. Il sera, suivant les cas, très faible ou très marqué. Chez un certain nombre d'animaux et surtout à l'état jeune, il n'est pas perceptible. On' sait, par exemple, qu'il est extrêmement difficile et pratiquement mème impossible de diagnostiquer à l'examen extérieur le sexe de beaucoup d'espèces de Passereaux, surtout à l'état jeune. Un des caractères sexuels secondaires les plus répandus est une diffé-rence de taille entre le mâle et la femelle adultes. Dans la généralité des cas, elle est faible et le mâle est un peu plus grand. Mais, chez divers Invertébrés et corrélativement à certains modes de vie, elle s'exagère et se renverse; on arrive alors au nanisme du mâle par rapport à la femelle. Le cas inverse est beaucoup plus rare. Les espèces qui offrent un dimorphisme aussi intense sont de celles qui produisent et incubent un grand nombre d'œufs. La femelle, de par cela même, atteint de grandes dimensions et sa vie fonctionnelle est longue, tandis que celle du mâle est éphémère. Quand le dimorphisme s'exagère ainsi, il ne se réduit pas à une différence de taille, mais la forme générale et les divers organes sont eux-mêmes affectés et, bien souvent, les deux sexes ont été d'abord pris pour deux animaux d'espèces et même de groupes très: différents.

Voyons quelques exemples particulièrement nets à cet égard.

Dimorphisme intense. Ses facteurs éthologiques. Nous avons déjà parlé, à l'occasion de l'hermaphrodisme, des Crustacés Isopodes Epicarides, qui vivent en parasites sur d'autres Crustacés. Le mâle reste nain et garde à peu près la forme larvaire. La femelle, au contraire, subit des déformations énor- 
mes, du fait de l'hypertrophie de son ovaire et des appendices lamelleux à l'intérieur desquels elle incubera les embryons; elle ne serait plus recomnaissable pour un Isopode, si on ne la rencontrait qu'à l'état adulte. Dans ce groupe si uniforme, les femelles
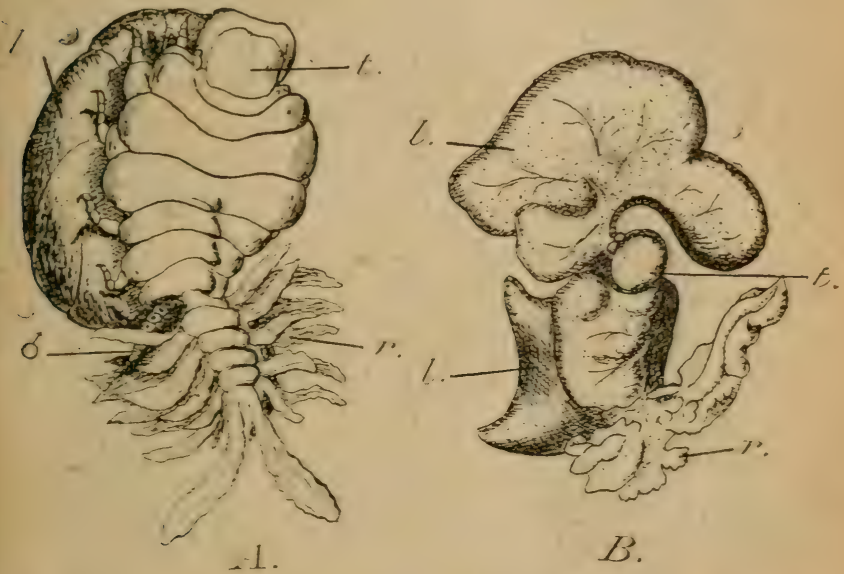

Fic. 18. - Deuz exemples dEpicarides Bopyriens, parasites des Crabes (dapris Giard et Bonnier).

A, Cepon elegans. Lianimal est três déformé, mais on reconnait encore les segments et les appendices; sur le côté de l'abdomen on aperçoit le mâle nain $\sigma^{*} ; B$. Portunion Tossmanni (Entonisciens). La femelle méconnaissable comme Isopode. Plusieurs mảles microscopiques sont généralement présentz au milieu des lamelles incubatrices; $t$, tète; $l$, lamelles incubatrices sur le thorax; $r$, appendices respiratoires' abdominaux.

d'Epicarides offrent une variété et une bizarrerie de formes déconeertantes, dont la figure 18 donnera une idée. Le mâle nain vit à demeure sur la femelle, sur laquelle il est plus ou moins mobile. (Cf. fig. 16, p. 45.).

Chez cette même classe des Crustacés, un autre 
ordre, celui des Copépodes, présente aussi de.nombreuses formes parasites sur des animaux très divers. On y retrouve, à tous les degrés, les mèmes phénomènes, avec une variété non moins extraordinaire, mais à laquelle je me borne à faire allusion.

Dans toute cette double série, le parasitisme est la cause évidente du dimorphisme, et comme, suivant

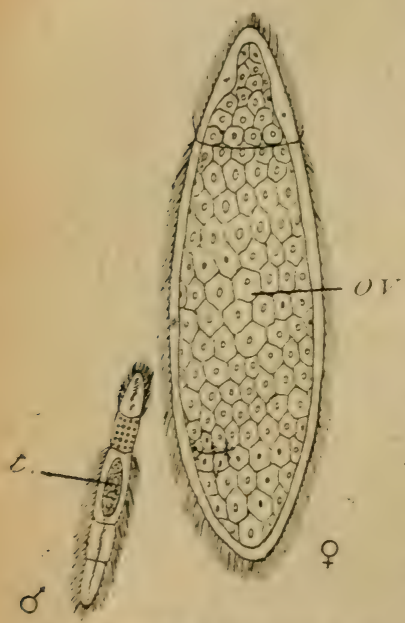

Fic. 19. - Les deur seres de Rliopalura ophiocomæ (Orthonectide), dans la yosition où se fait la fécondation.

$t$, testicule; $o v$, orules. les espèces considérées, celui-ci offre tous les degrés, on conçoit quelle merveilleuse illustration des faits de cet ordre il y a là pour la théorie transformiste.

Le parasitisme produit des effets analogues dans plusieurs autres groupes. Je citerai par exemple les Orthonectides, organismes tout à fait inférieurs qui vivent dans divers Invertébrés (Annélides, Echinodernes,etc.); ils ont été l'objet, au début, d'une méprise qui illustre bien l'intensité du imorphisme sexuel. On a décrit, en effet, tout d'abord comme deux organismes de genres différents

(sous les noms de Rhopalura ophiocomæe et d'Intoshia gigas) le mâle et la femelle d'une même espèce, tout à fait dissemblables de taille et d'aspect (fig. 19).

Il est à remarquer que, dans ces cas où l'on peut attribuer l'intensité du dimorphisme sexuel au parasitisme, l'hermaphrodisme voisine avec le dimor- 
phisme. Le parasitisme est facteur de l'un et de l'autre.

Ce double effet se retrouvera sous l'influence d'un autre état éthologique, la fixation à un substratum.

C'est ce que montre la Bonellie, ver marin, qui vit cramponné au fond des fentes de rochers. Longtemps on n'a connu que les femelles; mais on avait observé, dans la partie terminale de leurs voies génitales, un petit être microscopique que l'on avait considéré comme un organisme inférieur parasite. Or, il a été démontré depuis que c'était le mâle de l'espèce; il reste nain et la plupart de ses organes internes sont atrophiés, sauf les testicules; il passe toute son existence éphémère, comme celui des Epicarides, sur la femelle même.

Un dimorphisme sexuel aussi intense est presque toujours corrélatif d'un mode d'existence très spécial, tel que le parasitisme ou la vie fixée; mais ce n'est pas là une loi sans exception. On en trouve des exemples chez des formes vivant librement. Ainsi, c'est un cas très général dans le groupe des Rotifères et chez certains petits vers marins du genre Dinophilus, dont nous reparlerons à propos du déterminisme du sexe.

Chez les animaux à éthologie libre et normale, le dimorphisme sexuel peut encore être intense, sans porter sur une différence de taille ni de complexité organique générale. Il consiste alors en particularités tenant à l'ornementation extérieure. Chacun a présent à l'esprit le contraste entre le paon et la paonne, les différences entre le coq et la poule de bassecours (plumage, ergots, crêtes, etc.) Les oiseaux de paradis offrent au plus haut degré l'opposition d'un plumage terne chez la femelle, éclatant chez le mâle. Chez les Mammifères, il suffit de songer à la crinière du lion, aux bois du cerf qui manquent à la biche. 
Chez les Invertébrés, de nombreuses espèces sont aussi nettement dimorphes; je me bornerai à citer à cet égard les Insectes. Dans certains types, le mâle seul est ailé, la femelle reste aptère : tel est le cas universellement connu du Ver luisant (Lampyris noctiluca); on en trouve d'analogues chez les Papillons (Psyche, cf. fig. 32, p. 215, Orgyia, etc.) et dans d'autres groupes.

Chez la plupart des espèces, ce sont des différences
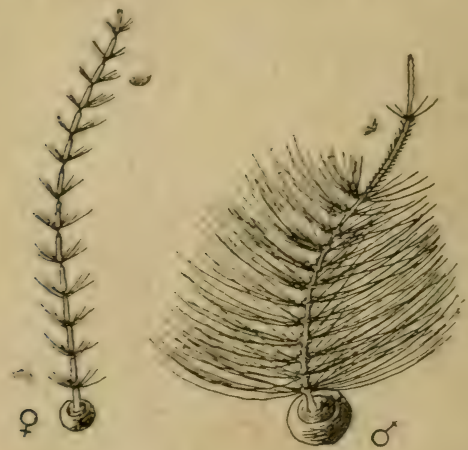

Fig. 20. - Anterne de Cousin (Cules) male (o) et femelie (f), montrant is différence dans les deux: sexes (d'après Mliall).

de couleur, d'ornementation ou de structure dans la carapace ou dans les appendices. Chez les Coléoptères de la famille des Lucanides, vulgairement CerísVolants, le mâle possède des mandibules beaucoup plus longues et plus robustes que la femelle. Le plus sourent les antennes diffèrent dans les deux sexes (fig. 20).

Le dimorphisme peut se traduire enfin par' des différences qui, pour n'être pas matérialisées extérieurement et n'être perceptibles que pendant la vie, n'en 
sont pas moins significatives. Telles sont celles qui sont relatives aux instincts, parfois très distincts chez ie màle et chez la femelle, ou à certaines aptitudes que l'un des deux sexes possède seul; tel est le chant, chez le mâle des Oiseaux, pour n'en citer qu'un exemple connu de tous.

Au reste, le nombre des faits relatifs au dimorphisme sexuel est infini et il faut nous borner ici à siønaler sa généralité, la variété de sa nature et de son intensité.

§ 3. - Polymorphisme sexuel. - Un dimorphisme sexuel plus ou moins accentué est la règle, mais il y a, dans plusieurs groupes du règne animal, des espèces qui offrent un réritable polymorphisme plus ou moins étendu des màles ou des femelles ${ }^{1}$.

C'est principalement chez les Insectes que des exemples de ce polymorphisme sexuel ont été mis en éridence. Ainsi, chez des Coléoptères Psélaphides du genre Bythinus, Peyerimhoff, en Algérie, a constaté qu'il existe des màles ailés et des màles aptères. Mais les exemples les plus remarquables et les plus classiques sont fournis par des Papillons. Le Papilio memnon, qui role à Jara. a une seule forme de màles et trois formes de femelles nettement différentes par le coloris des ailes; on leur a donné les noms de $P$. achates, $P$. agenor et $P$. laomedon; mais leur identité spécifique ressort du fait que toutes trois apparaissent dans des élerages issus d'une mème ponte. De mème, le $P$. polytes de Cerlan a trois formes de femelles dites $P$. polytes, $P$. aristolochiz et $P$. hector. Le Papilio merope en a quatre.

On a remarqué que ces diverses formes de femelles

1. On désigne parfois celte particularité sous les noms de

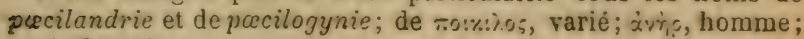
ชงทั่, femelle. 
ressemblaient parfois assez étroitement à des Papillons de groupes différents et on a voulu expliquer polymorphisme et ressemblance par la théorie du mimétisme, dont voici le principe. Les espèces imitées auraient toujours une saveur âcre, à cause de laquelle elles ne seraient pas mangées par les oiseaux. Les espèces qui les imitent seraient confondues par leurs ennemis avec elles et par suite épargnées. La ressemblance en question constituerait donc une protection et aurait été développée par la sélection naturelle. On est revenu aujourd'hui de ces explications par trop finalistes, qui ont toutefois le patronage de naturalistes illustres. La base de faits sur lesquels on prétendait les appuyer est plus que fragile 1 .

D'ailleurs, les ressemblances en question nous apparaissent et s'imposent à nous dans des collections, mais sont généralement loin d'être aussi frappantes dans la nature. Telles espèces, dont les ailes ont des colorations groupées en dessins analogues, volent de façons toutes différentes et se distinguent aisément de loin. D'ailleurs, la vue est loin d'être le seul guide des animaux dans la recherche de leurs proies; l'odorat doit les renseigner au moins autant. En examinant de près les choses, on voit que les ressemblances qui ont suggéré la théorie du mimétisme n'ont aucune efficacité protectrice véritable, et ce n'est pas par cette considération qu'il faut chercher à les expliquer?

\section{Le polymorphisme des Insectes sociaux. - Un}

1. Voir, à ce sujet, Et. Rabaud, Qu'est-ce que le Mimétisme? Revue $d u$ Mois, 1913. Cette question est également très bien traitée, au point de vue général, par Mic Atre. Proc. Acad. Natur. Science. Philadelphie, t. 64, 1912.

2. Un polymorphisme d'ordre tout différent existe chez des espèces qui se reproduisent tantôt parthẻnogénétiquement 
autre groupe de faits de polymorphisme sexuel se rencontre chez les Insectes sociaux, tels que les Termites, les Abeilles et les Fourmis. A côté des mâles et des femelles proprement dits, ces sociétés renferment d'autres individus stériles, par atrophie précoce des organes génitaux, les neutres, qui diffèrent morphologiquement des sexués. Cheż les Termites (fig. 21),
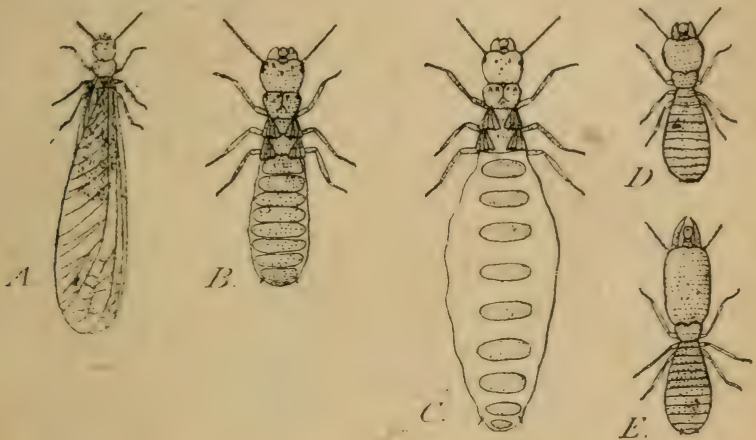

Fic. 21. - Polymorplisme d'un Termite (Termes lucifigus) (d'après Lespés). A. Sexıé ailé; $B$, Roi; $C$, Reine; $D$, ouvrier; $E$, soldat.

il y a gênéralement plusieurs formes de neutres bien distinctes : les uns, dits soldats, avec une tête et des mandibules très fortes, défendent la colonie; les autres, dits ouvrier's, assurent l'éducation des larves et la nutrition des sexués, mâle et femelle. C'est un problème encore très incomplètement résolu de savoir si les circonstances du début de la vie individuelle, les facteurs externes en un mot, suffi-

tantôt par œufs fécondés. Les femelles parthénogénétiques et les femelles fécondables sont en effet souvent séparées par des divergences morphologiques très nettes. Nous y reviendrons à propos de la parthénogénèse. 
sent à faire évoluer un embryon vers la forme sexuée ou neutre, ou si la destinée de chacun est fixée $a b$ ovo.

Le polymorphisme des Fourmis est du même ordre que celui des Termites; mais chez ceux-ci les neutres étaient des individus des deux sexes dont les glandes génitales avaient arorté; chez les Fourmis; au contraire, et chez les autres Hyménoptères sociaux, ce sont toujours exclusivement des femelles à ovaires rudimentaires. Dans certaines espèces de Fourmis, tous les neutres - qu'on appelle des ouvrières sont semblables entre eux; dans d'autres ils sont polymorphes, comme chez les Termites.

Enfin, chez les Abeilles, il y a aussi, dans la ruche, des mâles ou faux-bourdons, une femelle ou reine, et des neutres ou ourrières, qui sont encore des femelles stériles. Mais ici ces dernières sont ailées, alors que dans les cas précédents les neutres étaient aptères. L'érolution d'une larre femelle vers la forme reine ou ouvrière parait dépendre de la nourriture qu'elle reçoit au cours de son développement.

\$4. - Gynandromorphisme. - A cóté du dimorphisme ou du polymorphisme sexuel, phénomènes normaux, il faut faire une place à une manifestation exceptionnelle, à laquelle on a donné le nom de gynandromorphisme. C'est la juxtaposition en mosaïque, sur le même individu, de caractères mâles et de caractères femelles.

Nous avons déjà eu l'occasion d'en parler à propos de l'hernıaphrodisme chez les Mammifères et chez l'homme en particulier. Il est en effet généralement (mais non nécessairement) corrélatif d'un hermaphrodisme plus ou moins rudimentaire. Chez les Vertébrés, il est rare qu'il aille jusqu'à une mosaïque véritable de caractères des deux sexes. Il se borne le plus souvent à des malformations des organes 
sexuels, entraînant cependant parícis une altération générale des formes d'un sexe.

Il y a cependant des exemples où l'individu se présente comme une juxtaposition des parties mâles et femelles, au point de vue de l'aspect extérieur. Max Weber a observé, par exemple, un pinson qui arait le plumage du mâle à droite, celui de la femelle à gauche; l'anatomie interne correspondait à cette disposition : l'animal avait un testicule du côté droit et un ovaire à gauche.

Mais c'est chez les Arthropodes et surtout chez les Insectes que le gynandromorphisme proprement dit a été le plus rencontré, peut-ètre en raison du grand
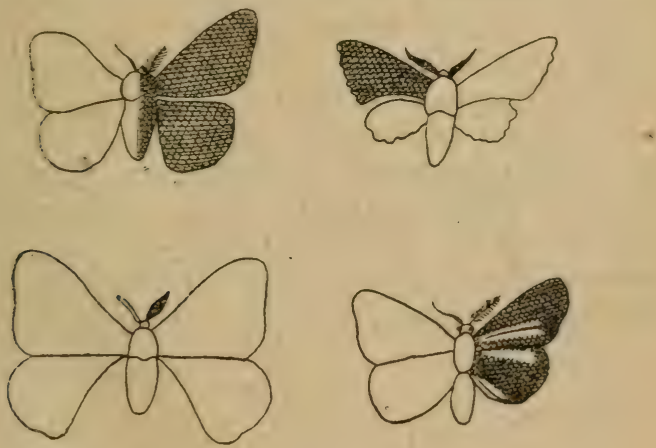

P̈ic. 22. - Eulémas de queliques cas de gynandromorphisme chez divers Papillons (diprés Wencke). Les parties ayant les caractères miles sont onhries, lis flarties ayant les caracteres femelles sont laissées en hlane.

nombre d'individus étudiés arec soin par les collectionneurs. En 1904, on pouvait relever 909 cas publiés, rien que chez les Papillons, et se rapportant à 211 espèces. Les caractères sexuels secondaires les plus saillants chez les Insectes sont dans la forme des antennes, dans la structure des yeux, dans la 
coloration des ailes, dans l'armure génitale. Les cas de gynandromorphisme connus montrent une très grande variété; dans certains d'entre eux, le plan de symétrie sépare rigoureusement une moitiẻ mâle et une moitié femelle. Mais dans beaucoup e'est une disposition beaucoup moins régulière (fig. 22$)$. Une antenne ou une aile seulement seront anormales; parfois il y aura une panachure complexe des caractères des deux sexes. Un petit nombre de ces Papillons gynandromorphes - 15 seulement - ont été étudiés anatomiquement d'une façon précise; partout les conduits génitaux étaient plus ou moins anormaux, participant à la fois des deux sexes. Quant aux glandes génitales elles-mêmes, dans quatre cas il y avait hermaphrodisme; dans trois elles étaient mâles; dans huit elles étaient femelles. On peut donc dire que le gynandromorphisme chez les Insectes est généralement accompagné d'anomalies des voies et des glandes génitales, mais il n'y a pas de correspondance rigoureuse entre l'aspect extérieur et les dispositions des parties internes. Pour le moment, bornons-nous à enregistrer le fait. Nous verrons ultérieurement de quelle interprétation il est susceptible. 


\section{CHAPITRE VII}

\section{LES CARACTÈRES SEXUELS SECONDAIRES \\ LA REPRODUCTION. - LA SÉLECTION SEXUELLE}

Caractères sexuels secondaires en rapport direct avec la reproduction, ou aidant à la rencontre des sexes. - Exagération du dimorphisme sexuel dans les périodes de reproduction.

Le dimorphisme sexuel peut-il s'expliquer par la sélection naturelle? - Théorie de la sélection sexuelle. - Sa critique.

$\$ 1$ - Les divers éléments du dimorphisme sexuel sont évidemment en corrélation étroite avec le sexe; mais leur rapport arec la fonction génitale et la reproduction proprement dite est plus ou moins direct et plus ou moins difficile à saisir.

Caractères en rappoit direct avec la reproduction. - Un certain nombre de dispositions spéciales aux femelles servent à assurer l'élevage des jeunes; telles sont les glandes mammaires des Mammifères, telle la poche marsupiale chez les Marsupiaux, où les jeunes sont blottis pendant l'allaitement, telles les lamelles incubatrices formant une poche qui joue un rôle analogue chez de nombreux Crustacés 1 . On peut ajouter que, dans certains cas, d'ailleurs exceptionnels, c'est au màle qu'est dévolue une fonction de

1. Voy. fig. 18, p. 67. 
cet ordre; il en est ainsi de certains Crapauds, comme le Pipa, chez lesquels les œufs pondus sont greffés en quelque sorte sur le dos du mâle, des Poissons lophobranches (Syngnathes et Hippocampes) où le mâle possède une véritable poche incubatrice ventrale, des Pycnogonides où le mâle également porte les embryons en roie de développement sur une paire de pattes supplémentaire.

Chez les Crustacés, on observe d'autres faits de dimorphisme sexuel relatifs aux appendices et en

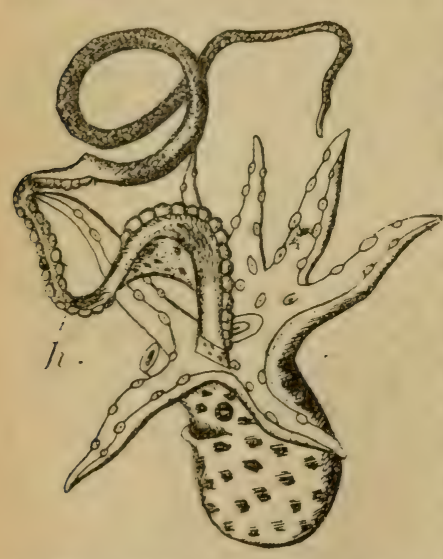

FIG. 23. - Mále d’Argonauta argn montrant le bras hectocotylisé $h$ avec sa lanière déroulée. rapport avec l'accouplement. Les deux premières paires de pattes abdominales, chez la femelle des formes supérieures, les Décapodes (Homards, Ecrevisses, Crabes, etc.) sont plus ou moins rudimentaires, tandis que chez le mâle elles sont différenciées en stylets copulateurs à l'aide desquels le sperme est introduit dans les voies génitales femelles. C'est à une fonction analogue que correspond une très curieuse particularité qu'offrent un

certain nombre de Mollusques Céphalopodes, tels que les Pieurres et en particulier l'Argonaute (fig. 23). Les huit bras de la femelle sont semblables entre eux. Chez le mâle, au contraire, l'un de ces bras, que l'on nomme l'hectocotyle, a une forme spéciale et se prolonge en une longue lanière; il présente ane gouttière où s'accumulent les spermatophores et c'est par 
l'intermédiaire de ce bras que le mâle féconde la femelle. Chez certaines espices même, le mâle, ampute spontanément, ou comme on dit, autolomise ce bras lors de l'accouplement et le laisse tout chargé de spermatophores dans le sac palléal de la femelle.

Cet exemple montre déjà combien les rapports des particularités du dimorphisme sexuel arec l'accouplement ou la reproduction sont inattendus. Dans beaucoup de cas il en est ainsi et on ne peut constater ces rapports que par des observations précises. Ainsi chez beaucoup de Crustacés, et en particulier de Copépodes pélagiques, les antennes sont beaucoup plus longues chez le mâle. Elles servent à celui-ci lors de l'accouplement, à maintenir la femelle.

Caractères pouvant faciliter la rencontre des sexes. - D'autres fois le rapport est moins direct ou plutó moins matériel; les différences entre mâle et femelle peuvent ètre considérées cependant comme facilitant la rencontre des sexes.

Il en est ainsi fréquemment quand l'un des sexes ćmet des odeurs, ou bien possède des organes olfactifs capables d'en percevoir. On a des exemple du premier cas, chez divers Mammifères, comme le Cherrotin porte-musc, les Cervidés, le Castor et bien d'autres. Le dimorphisme des antennes de beaucoup d'Insectes, comme les Hannetons, les Bombyx, parmi les Papillons, correspondent vraisemblablement au second.

La présence d'une seule femelle de certains Bombyciens, comme le grand Paon de nuit ou le Bombyx du chêne. suffit à attirer de très loin un grand nombre de mâles, et l'on ne peut guère s'expliquer le fait que par l'émission d'une odeur par la femelle, quoique nous ne la percevions pas nous-mêmes. Fabre a fai un tableau, très pittoresque à son habitude, d'expé- 
riences à ce sujet. Tuutefois il n'a pas réussi à cons. tater d'une façon bien convaincante que, privés d€ leurs antennes, les mâles cessent d'être attirés et il a hésité à conclure formellement que leur attraction soit déterminée par des sensations olfactives perçues grâce aux antennes. Il envisage la possibilité bien aventureuse de radiations attractives spéciales émises par la femelle, et auxquelles nous ne serions pas sensibles. L'hypothèse d'un phénomène olfactif reste la plus vraisemblable, mais on roit qu'il est malaisé d'établir rigoureusement des faits de ce genre.

Dans le groupe des Papillons, on connait, d'autre part, des émissions d'odeurs très caractérisées constituant des faits de dimorphisme sexuel, mais qui ne peuvent relever de l'explication prêcédente. C'est Fritz Müller qui a attiré l'attention sur ces propriétés, chez des espèces brésiliennes; certaines d'entre elles ont un parfum aussi accentué que des fleurs et on a reconnu qu'il émane de glandes unicellulaires situées - sur les écailles des ailes et produisant des éthers. Parmi les espèces de nos régions, le Sphinx du Liseron a une odeur de musc; le Pieris napi, celle de l'essence de mélisse ou de citron. Ce seraient surtout les Papillons de nuit aux couleurs ternes qui seraient odorants, tandis que les formes diurnes aux couleurs vives ne le seraient pas. Les glandes parfumées n'existeraient que chez les mâles et pas chez les femelles. On ne peut donc voir dans ces odeurs, cette fois très caractérisées, un moyen d'attraction des mâles comme précédemment. Weismann a rattaché leur production à la théorie de la sélection sexuelle que nous examinerons un peu plus loin.

Le rapprochement de toutes ces données montre en tout cas qu'il faut contrôler soigneusement toutes les hypothèses faites au sujet des odeurs.

Comme l'olfaction, la vision est évidemment un des 
moyens principaux que les individus d'une espèce ont de se reconnaitre. Les différences de livrée entre les sexes ont été interprétées avec beaucoup de vraisemblance comme étant un signe de reconnaissance entre eux.

De mème, dans certains cas au moins, la luminosité. Chez le Ver luisant, par exemple, il n'y a guère de doute que la rencontre des sexes ne soit favorisée par la luminosité particulière de la femelle.

De mème, les bruits émis par l'un des sexes sont aussi un moyen d'appel et l'on a noté depuis longtemps que le chant, parmi les Oiseaux, est surtout l'apanage des mâles et qu'il est particulièrement développé chez les espèces à livrée terne; il est rudimentaire au contraire chez celles où le mâle a une parure brillante.

Ainsi beaucoup de dispositions qui, au premier abord, paraissent tout à fait étrangères à la fonction reproductrice, s'y rattachent en réalité indirectement et méritent, physiologiquement comme morphologiquement, la désignation de caractères sexuels secondaires. Cette interprétation est encore confirmée par la constatation que beaucoup de ces caractères sont renforcés à l'époque des amours ou même n'existent qu'alors.

Déjà, d'une façon générale, le dimorphisme sexuel n'est réalisé, au moins dans sa totalité, qu'à partir de l'àge de la reproduction. Il fait défaut ou est rudimentaire chez les individus jeunes. Mais il y a. des exemples nombreux et très variés du développement périodique des caractères sexuels secondaires pendant la saison où s'unissent les sexes. Le chant du Rossignol, les coassements des Grenouilles, les stridulations des Grillons et des Cigales, en sont des exemples bien connus. Non moins caractéristiques sont les variations dans la livrée, auxquelles on a 
donné le nom sisnificatif de parure de noces. Le mâle surtout, au moment de la reproduction, acquiert une livrée plus brillante. Tel est le cas classique les Combattants (Machetes pugnax), dont la gorge est recouverte alors d'une haute fraise de plumes. Chez les Batraciens. ciest au printemps que les pouces des mâles montrent bien déreloppée la callosité qui joue un rôle certain dans l'embrassement de la femelle ${ }^{1}$; c'est alors aussi que certains Tritons présentent des couleurs rives et la crête dorsale 'ui disparait ensuite; chez certains Poissons même, le mảle se colore, à ce moment, de teintes plus vives. Ces deux derniers exemples montrent que ces phénomènes ne sont pas nécessairement liés aux circonstances de la rencontre des sexes; car chez les Poissons en question, il n'y a pas d'accouplement.

Gardons-nous, en cette matière, comme en beaucoup d'autres, d'un finalisme aussi séduisant que vain. $\mathrm{Si}$ beaucoup de caractires sexuels jouent un rôle accessoire plus ou moins précis dans la reproduction, il n'est pas nécessaire qu'il en soit de mème pour tous et il ne faut pas imaginer que leur raison d'être soit la reproduction même.

5. - La théorie de la sélection sexuelle. - La théorie darwinienne de la sélection naturelle, qui, en renversant le finalisme ancien, a cependant conservé à la Biologie une tournure finaliste, n'a pas érité cette erreur. La preuve en est l'explication que Darwin a rherché à donner des caractères sexuels secondaires, dans la théorie de la sélection sexuelle.

La sélection naturelle et les caractères sexuels. La sélection naturelle serait interrenue dans la diffé-

1. Voy. fig. 26, p. 109. 
renciation des sexes, sous sa forme générale, en conservant et amplifiant les variations propres à chacun d'eux et qui étaient avantageuses à l'individu, étant données les conditions de vie particulières au mâle ou à la femelle, ou les nécessités propres à l'un et à l'autre dans la concurrence vitale. On constate, par exemple, que dans plusieurs groupes, la lirrée de la femelle est moins brillante que celle du mâle et qu'elle s'harmonise avec les couleurs du milieu. Beaucoup de femelles sont homochromiques et de ce fait clles seraient mieux protégées contre leurs ennemis. Ceite protection assurerait dans une plus large mesure la naissance et l'élevage de la progéniture. La sélection naturelle aurait donc développé l'homochromie du sexe femelle, c'est-à-dire conservé et accentué un élément de dimorphisme sexuel.

De mème le dimorphisme si accentué de beaucoup de parasites trouverait son explication de façon analogue. La fécondité énorme de ces parasites, l'incubation si fréquente de leurs embryons, apparaissent dans la théorie de la sélection, comme des dispositions que celle-ci a déreloppées et qui compensent la destruction d'innombrables larves. Aux chances de destruction communes à toutes les espèces pendant leur développement, s'ajoute en effet pour les parasites la nécessité de rencontrer à un stade donné l'hôte dans lequel ils peuvent continuer à évoluer, faute de quoi ils périssent; cette condition n'est satisfaite que pour une minorité infime. L'hypertrophie et les déformations si marquées de la femelle, le grand développement, chez elle, des organes d'incubation résulteraient de l'action de la sélection qui aurait agi d'une façon opposée sur le mâle. Le nanisme de celui-ci et sa vie sédentaire sur la femelle, assurent en eff t la fécondation; sa maturité mème, atteinte de façou précoce, à un stade encore larvaire, garantit un rôle 
effectif a un plus grand nombre d'individus et par suite favorise encore la conserration de l'especce. Tel est le genre darguments par lesquels on fait intervenir la selection naturelle dans lexplication du dimorphisme sexuel. Charpus cas exigerait une cri. tique spéciale.

La selection sexuelle. - Mais Darwin a imaginé. en outre, un roble tout particulier de la sélection que nous allons envisager maintenant : la sélection sexuelle.

Elle s'exercerait entre les individus de l'espèce pour arriver à la reproduction, comme la sélection générale s'exerce entre tous les tres pour subsister. Elle résulterait de la concurrence entre les màles pour la possession des femelles. Celles-ci choisinaient les màles les mieux doués; les autres seraient éliminés de la reproduction, qui, prar suite, perpétuerait et lévelopperait la qualité des individus élus. Un grand nombre de caractères sexuels secondaires résulteraient ainsi du choix des mâles opéré par les femelles.

Quelle est la base de faits sur laquelle s'appuie cette thérie? Et d'abord y a-t-il dans la nature une concurrence entre les máles pour le choix des femelles? Cette concurrence ne peut véritablement exister que si les premiers sont en nombre trop considérable. Nous verrons plus loin que, le plus souvent, les deux sexes sont représentés par des nombres sensiblement Egaux d'individus : d'autres fois il y a un grand excès de l'un des sexes. Chez certaines Ephémères, par exemple, on estime qu'il y a soixante máles pour une femelle. Mais il est bien évident qu'ici l'équilibre véritable dépend du nombre d'indiridus d'un sexe qui correspond au fonctionnement sexuel normal de l'autre. La question n'est donc pas susceptible d'une -éponse générale, mais doit être examinée pour chaque 
type, en tenant compte de ses mœurs particulières.

En réalité, chez un certain nombre d'animaux supé. rieurs, Mammifères ou Oiseaux, on constate de façon authentique une lutte des mâles entre enx à l'époque du rut, et c'est aux survivants, c'est-ì-dire aux plus forts, qu'appartiennent les femelles. Les mâles des Cerfs se livrent ainsi des combats dans lesquels leur ramure est leur arme principale. Comme c'est un caractère sexuel secondaire, on peut admettre ici l'intervention de la sélection sexuelle. Les combats entre måles, chez beaucoup d'Oiseaux, sont non moins réels.

Mais si l'explication estainsi plausible pour certains cas particuliers (sous réserve des objections générales faites à la théorie de la sélection naturelle), il est dangereux de l'étendre à la légère. Il importe de constater effectivement la lutte des males entre eux et non pas seulement de la supposer d'après certaines structures particulières qui pourraient servir d'armes. On a vu parfois dans les mandibules si développées chez les mâles de certains Coléoptères, comme les Lucanes ou cerfs-volants pour ne citer que cet exemple, une arme de lutte analogue aux bois des Cervidés. En réalité aucune obserration n'a montré la rlalité d'une lutte entre les mâles, lutte oủ ces appendices seraient des armes.

Par contre il a été constaté, chez d'autres Coléoplères, où existe un dimorphisme analogue, tels que les Dynastes (fig. 24), que les mâles luttaient entre eux et que leurs appendices frontaux, dont sont dépourvues les femelles, leur servaient effectivement dans ces combats. Picado à observé le fait à CostaRica. On trouve souvent sur le théâtre de ces luttes les débris des mâles vaincus.

La concurrence entre les mâles peut évidemment ne pas revêtir toujours la forme d'un combat propre- 
ment dit. Si la recherche de la femelle est sous la dépendance de sensations olfactives, le mâle le mieux doué pour l'odorat aura chance d'atteindre plus tôt le but, et l'acuité de l'odorat, caractère sexuel secon daire, relèvera du mécanisme possible de la sélection

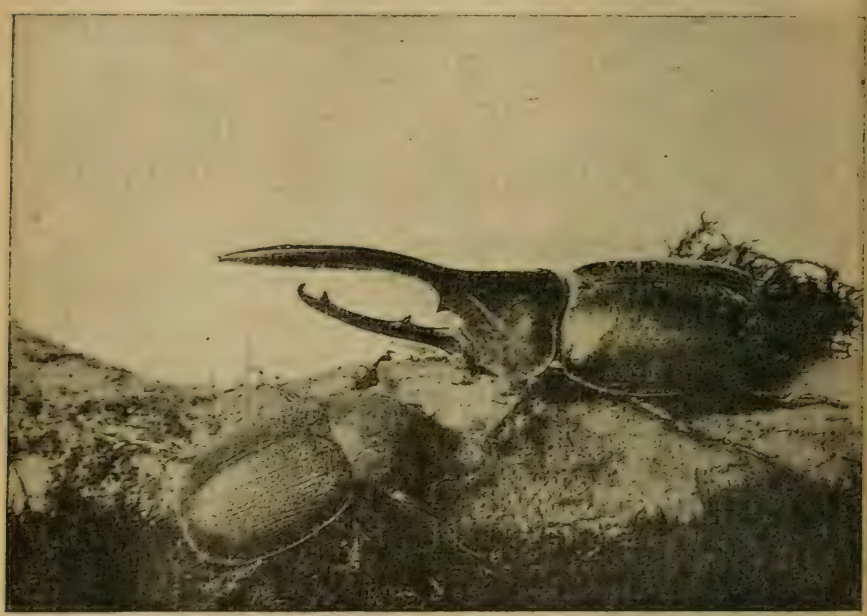

Fic. 2'. - Màle et femelle de Dynastes hereules. (Cliché Picado.) (3/8 grand.

sexuelle. D'autres raisonnements u même genre sont évidemment concevables.

Dans des faits de l'ordre des précédents, la théorie de la sélection sexuelle n'apparaît tout au plus que comme une explication partielle.

Elle est bien plus contestable encore dans les cas auxquels Darwin, Weismann, Poulton et bien d'autres, l'ont appliquée surtout. L. Plate a fait d'une façon particulièrement serrée la critique de ces idées. 
Les femelles de beaucoup d'espèces exerceraient, d'après ces biologistes, entre ces mâles, un choix véritable basé sur des raisons d'ordre esthétique. Elles accorderaient la préférence au mâle le plus beau par sa livrée, ou le plus habile chanteur, ou à celui qui exécuterait le mieux certaines parades. Les caractères de beauté seraient ainsi sélectionnés par les femelles.

Il est certain que dans un certain nombre d'espèces, particulièrement chez les Oiseaux, le chant, les danses effectuées par les mâles, l'érection de certains appareils placés sur la tête, comme on en a signalé dans quelques formes tropicales, attirent l'attention de la femelle et le fait que ces diverses manifestations s'exagèrent à l'époque du rut, suggère qu'elles sont très vraisemblablement une excitation ì l'accouplement. Elles peuvent donc relerer du mécanisme de la sélection, à supposer que celle-ci soit efficace.

Mais, tout d'abord, il est hardi et au fond injustifié d'admettre que l'impression de beauté que nous produit telle ou telle disposition, soit ressentie par des animaux. C'est là chose essentiellement subjective.

En outre, la réalité d'un choix du mâle par les femelles, en dehors de certains cas exceptionnels, est fort problématique. Mème chez les Oiseaux où les facultés psychiques sont incomparablement plus développées que chez les Insectes, les observations les plus objectives montrent que le plus sourent la femelle subit passivement le màle. A plus forte raison peut-on douter d'un choix du mâle le plus brillant chez les Papillons. La femelle peut-elle au reste juger du coloris de l'ensemble des ailes? Et le pourrait-elle, son choix serait-il basé sur les préférences que nous lui attribuons avec notre esthétique propre? D'ailleurs, chez les espèces dont les ailes ont les couleurs 
les plus rives, on a observé des cas nombreux oủ les màles, au moment de l'accouplement, ont déjà perdu leurs écailles, c'est-à-dire les éléments principaux de leur parure. Seitz, en particulier, en a fait la constatation, maintes fois, à Ceylan.

Pourrait-on citer, chez les Papillons, une observation décisive d'un choix par la femelle? Et par contre, les observations négatives sont très nombreuses. Nayer et Soule ont peint, par exemple, en rouge ou en vert, les ailes de nombreux mâles de Callosamia promethea. Malgré ce déguisement, ces mâles se sont accouplés aussi aisément que les normaux. Une autre expérience des mêmes auteurs tendrait cependant à accorder une certaine importance à la vision des femelles. Opérant en effet sur un Bombycien (Liparis dispar), ils ont coupé les ailes des mâles; les individus mutilés rẻussirent plus rarement à s'accoupler que les normaux; cela peut s'expliquer parce qu'ils étaient moins en état d'immobiliser les femelles; mais si l'on aveuglait celles-ci par un vernis opaque appliqué sur les yeux, les mâles privés d'ailes s'accouplaient aussi aisément que les autres.

Quand bien mème il existerait quelquefois, à un certain degré, un choix du mâle par la femelle, les faits obligent à déclarer très exagérée l'affirmation de Darwin d'après laquelle ce choix serait " une loi presque aussi générale que le désir du mâle "1. Les observations positives indéniables sont au contraire des plus restreintes. C'est l'idée théorique de la sélection sexuelle qui, par un cercle vicieux, au reste presque constant dans les spéculations sur la sélection en général, a suggéré les prétendues constatations de choix exercés par les femelles. La phrase

1. Origine de l'homme et Sélection sexuelle. 
suivante de Weismann ${ }^{1}$ montre bien cette déformation du raisonnement : "Il me parait indiqué, dit-il, daccepter le processus de la sélection sexuelie comme véritablement efficace et, au lieu de le mettre en doute, parce qu'on n'a que rarement l'occasion de constater le choix des femelles, il faut au contraire conclure de l'existence de nombreux caractères sexuels secondaires des mâles, dont la seule signification possible est la compétition amoureuse, que les femelles des espèces considérées sont sensibles à des dispositions de ce genre et font véritablement un choix ». On proclame généralement que la théorie darwinienne a éliminé le finalisme de la biologie; on roit par cette citation à quel degré, au contraire, liidée a priori de sélection a développé, chez des hommes de science contemporains, les tendances finalistes. Toute particularité morphologique doit correspondre à une fin, que l'on imagine d'une façon entièrement subjective.

Je rapprocherai de la citation précédente celle d'un éminent naturaliste anglais, Poulton 2; la doctrine de la sélection l'a conduit aussi à des interprétations beaucoup trop finalistes, notamment à propos du mimétisme. Il est de ceux qui défendent encore aujourd'hui la sélection sexuelle, et, à propos d'un Papillon (Charaeus graminis), il décrit les ébats de nombreux mâles autour d'une femelle en apparence passive (appar ontly motionsless); finalement, un mâle s'en empare, les autres alors s'éloignent et disparaissent. Rien donc, dans cette observation, n'indique un choix actif de la part de la femelle. Cependant Poulton ajoute: "Quand on observe ces ébats remarquables et compliqués, on est forcé de conclure que la

175).

'. Vorträge über Deszendenztheorie, I, 1902 (2e édit., pp. 1ił-

2. The Colours of animals, 1890, p. 291. 
femelle indique ses intentions d'une manière qui nous est inconnue et que pour les mâles c'est affaire d'honneur de suivre sa décision.. Le fait est bien connu des entomologistes et rient à l'appui de la théorie de Darwin ".

Est-il sûr qu'il $y$ ait dans des cas de ce genre autre chose que le pur hasard? Mais, en tout, cas, où est la raison de voir là un choix de la femelle et surtout un choix discerne et consenti jar les autres màles? Des exemples tels que ceux-là montrent que - si la théorie de la sélection a stimulé l'obserrateur, elle a exercé une influence pernicieuse sur sa mentalité et sa méthode. L'autosugrgestion se substitue graduellement à l'observation des faits ou tout au moins dénature l'interprétation à en tirer.

Nous arrivons donc à la conclusion que la sélection sexuelle, telle qu'elle a été conçue par Darwin et déveloprée encore récemment par Weismann et Poulton, ne peut être considérée comme rendant compte d'une manière générale de la différenciation des caractères sexuels secondaires. Il lui manque, à beaucoup près, une base objective de faits suffisande. 


\section{CHAPITRE VIII}

\section{LES CARACTĖRES SEXUELS SECONDAIRES}

\section{ET L'ACTIVITÉ FONCTIONNELLE DE L'ORGANISME}

Mélabolisme dans les deux sexes et réalisation du type spécifique. - La castration nutriciale chez les Guêpes (P. Marchal) et les neutres des Insectes sociaux.

Je dirai encore quelques mots d'une interprétation sénérale des caractères sexuels secondaires qui ne vise pas, à proprement parler, à ètre une explication de leur genèse, mais qui les ramène à une conception générale intéressante, quoique discutable.

Le métabolisme est en rapport avec le sexe. - Suirant que l'organisme élabore des œufs ou des spermatozoïdes, les transformations chimiques des substances qu'il assimile, en particulier la composition du sang, doivent, en principe, présenter une certaine différence.

Le métabolisme, comme on dit, ne doit pas être identique dans les deux sexes : la différence peut ètre insignifiante chez beaucoup d'animaux, mais on connait des cas où elle est notable. Vaney et Maignon ont montré, il y a déjà quelques années, que pendant toute la période de chrysalide, chez le ver à soie, les. femelles renferment toujours plus de glycogène et moins de graisse que les mâles. Maignon a constaté, 
d'autre part, chez le cobaye, que la teneur des muscles en glycogène est plus forte chez les mâles, et que la castration nivelle cet écart entre les sexes; ses expériences prouvent que l'activité nutritive est sous la dépendance directe des glandes génitales. Il résulte aussi des recherches plus récentes de G. Smith, que la teneur en graisse du sang des Crabes n'est pas la même chez les mâles et chez les femelles. Tout récemment encore, Steche a constatẻ que, chez les larves de certaines Tenthrèdes, le sang n'arait pas la même couleur dans les deux sexes: il semble que la chlorophylle des feuilles dont se nourrissent ces larres soit inégalement résorbée par la paroi intestinale des mâles et des femelles.

On peut évidemment admettre, d'une manière générale, que l'élaboration des œufs dans l'ovaire ou les soins donnés aux jeunes, absorbent dans le fonctionnement vital du sexe femelle une quantité d'énergie qui est disponible pour d'autres objets dans le sexe màle. On a alors émis l'hypothèse que, chez celui-ci, cet excès serait employé à un développement plus complet du type spécifique. Le mâle représenterait done, dans l'évolution, une étape plus avancée que la femelle; celle-ci, dans l'avenir, se rapprocherait de ce que le mâle nous présente aujourd'hui.

Cette conception me parait fortement entachée de métaphysique. Elle suppose que les espèces évoluent suivant une direction déterminée; c'est ce qu'on a appelé l'orthogénèse. On ņe peut nier que des faits assez nombreux s'accordent bien avec cette idée. mais de là à y voir un principe général, et a priori, il y a loin. Le point de départ lui-même est-il solide? Est-il sûr que la fonction génitale exige chez la femelle une consommation d'énergie plus considérable que chez le mâle? Un orule représente certainement une élaboration de substances énorme par rapport à celles 
qu'exige un spermatozö̈de; mais le nombre immense de ceux-ci compense peut-être, et au delà, les exigrences de chaque orule. Nous n'avons actuellement aucune donnée numérique qui nous permette de dire de quel côté pencherait la balance. D'ailleurs, si même la vie de l'ovaire exigeait plus d'énergie que celle du testicule, on trouverait peut-être dans les conditions de la nutrition chez la femelle des mécanismes contpensateurs, sans que sa morphologie dủt en être finalement influencée.

En réalité, nous nous trourons en présence de phéncmènes extrêmement complexes et à peu près totalement inexplorés, si on les envisage au point de rue énergétique; on leur applique néanmoins, dès à présent, des affirmations dépourvues de toute précision et de toute base solide, d'où on tire ensuite des conséquences générales.

La castration nutriciale chez les Insectes sociaux. - Dans cet ordre d'idées pourtant, quelques suggestions mieux étayées ont été formulées au sujet de l'origine des neutres chez les Insectes socraux, problème du même ordre que celui de la différenciation morphologique des sexes en général.

Cette question a servi de thème de virtuosité aux protagonistes des divers systèmes transformistes. Darwin avait déjà senti la difficulté d'expliq. -r par la sélection la formation d'un type spécial de neutres qui, ne se reproduisant pas, ne peuvent transmettre les modifications, mème arantageuses, qu'ils présentent. H. Spencer et Weismann ont, avec beaucoup d'ingéniosité, cherché à y voir une preuve ou une objection insurmontable ris-à-vis du lamarckisme ou du darwinisme.

A ces discussions a priori, il est plus fécond de substituer des observations précises, e $e^{2}$, si possible, 
de réritables expériences. Ciest à quoi, dans une certaine mesure, ont réussi les recherches de P. Marchal sur le Guêpes. La différenciation des neutres est encore à ses débuts chez ces Hyménoptères et peut indiquer par quelles roies ont été atteints des états d'évolution plus avancés, tels que ceux des Fourmis et des Abeilles.

Chez les Guêpes, en effet, les neutres (ouvrières) n'ont pas de caractères morphologiques tranchés qui les séparent des femelles véritables. Elles sont seulement plus petites. Elles peurent aussi plus facilement devenir fécondes que chez les Abeilles.

Dans une socièté de Guêpes, à l'automne, tous les mâles et les ouvrières meurent. Seules, les femelles fécondées survivent et chacune reconstitue un nid au printemps suivant: Des premiers œufs pondus par elles, naissent des ouvrières, petites, rabougries et, naturellement, stériles, que la mère a nourries et álevées elle-mème. Puis, ces premières ouvrières soignent les larves nées après leur propre éclosion. Pendant tout le printemps la population du nid s'accroît par la ponte incessante de la mère ou reine, mais ne comprend que des ourrières. Les mâles apparaissent seulement en été et les femelles fécondes vers la fin de la saison, où a lieı l'accouplement entre les sexes.

Marchal a isolé un certain nombre d'ouvrières dans une cage, avec un fragment de rayon et de la nourriture, mais sans aucune larve qu'elles eussent à élever. Il a constaté alors que ces ourrières, jusque-là stériles, deriennent fécondes et qu'elles pondent exclusivement des mâles ${ }^{1}$.

La fécondité des ouvrières réapparait donc si l'on

1. Comme ces ouvrières étaient parthénogénétiques, ce fait est un argument à l'appui de la théorie de Dzierzon, que nous étudierons plus loin. V. p. 167. 
suoprime leur fonction normale de nourrices des larves. On peut par suite admettre, arec beaucoup de vraisemblance, que leur stérilité était la conséquence de cette fonction; leurs oraires se déreloppent, en effet, dès qu'elles peuvent résorber pour elles-mêmes la nourriture qu'elles fournissaient jusque là aux larves. La stérilité des ouvrières, chez les Guèpes, semble donc bien être, suivant l'expression de Marchal, un phénomène de castration nutriciale.

Dans les sociétés d'Hyménoptères plus différenciées, telles que celles des Abeilles, on sait que les futures reines reçoirent, dès l'état larvaire, une nourriture spéciale, la gelée royale, dont les ouvrières et les mâles, élevés dans les alvẻoles ordinaires, sont privés. Il y aurait donc là, pour les ouvrières, une première cause de castration que l'on peut appeler la castration alimentaire, à laquelle la castration nutriciale se superposerait ensuite. Cette dernière aurait une influence d'autant plus manifeste que le degré d'érolution sociale est moins élevé; dans les sociètés très différenciées, en effet, la castration alimentaire agissant sur la larve suffit et produit un effet, en général, définitif ${ }^{1}$.

Le mécanisme précédent rend compte de la différenciation physiologique des neutres : comment y rattacher leur différenciation morphologique? Chez les Guêpes, elle est très faible encore et consiste seulement dans une différence de taille, qui va en s'atténuant au fur et à mesure que la saison avance et que la fonction nutriciale est moins intense, par suite de l'accroissement du nombre des nourrices. En même

1. On sait cependant que, dans certains cas, en l'absence de reine, les ouvrières d'Abeilles deviennent fécondes et pondent des màles; mais c'est un fait assez exceptionnel, tandis que la fécondité des ouvrières de Guêpes dans des conditions analogues semble se produire régulièrement: 
temps, la stérilité s'atténue. Finalement, en été, les causes qui l'avaient provoquée sont annihilées et les larres deviennent des femelles fécondes. Les œufs qui les produisent se sont développés pendant la période chaude de l'année.

Ces phénomènes se répètent à chaque génération. Les femelles fécondes se développent en été, c'est-àdire dans des conditions ambiantes très différentes de celles que trouvent les œufs d'où dérivent les ouvrières au printemps suivant.

Marchal admet que, sous l'influence de ces conditions extérieures estivales, les propriétés de l'œuf sont peu à peu modifiées et que, par ces modifications mêmes, le contraste s'accentue entre les propriétés héréditaires de l'œuf et les çonditions extérieures où se déreloppe ensuite l'ouvr̂̉ère. L'ouvrière est, dit-il, le résultat d'une expérience naturelle de tératogénie répétée indéfiniment et avec une intensité progressive. Il est naturel d'admettre que ces effets soient également progressifs et que, peu à peu, le type des ouvrières s'écarte de celui des femelles fécondes, par le désaccord toujours grandissant entre les propriétés héréditaires de l'œuf qui les produit et des circonstances où il érolue, sans que cependant l'hérédité ait eu à interrenir directement.

Ce n'est là encore qu'un essai d'explication auquel, en dépit de son ingéniosité, bien des objections peuvent être faites; mais il a le mérite de placer la discussion sur un terrain expérimental, où le progrès est possible quoique très difficile.

Si loin que l'on soit du but, ces tentatives mêmes montrent combien les problèmes auxquels elles se rapportent sont complexes et par oủ il convient de les aborde: 


\section{CHAPITRE IX}

\section{RAPPORTS ENTRE LES GLANDES GÉNITALES \\ ET LES CARACTĖRES SEXUELS SECONDAIRES CASTRATION ET TRANSPLANTATION}

La castration par sénilité : arrhẻnoïdie et thẻlyidie. — Castration parasitaire. - Castration expérimentale; les eunuques.

La castration chez les Vertébrés altère les caractères sexuels secondaires. - Résultats négatifs des expériences faites sur les Insectes.

La transplantation des glandes gẻnitales : elle affaiblit ou annule les effets de la castration. - Expériences faites sur le Coq, sur la Grenouille. - Expérience de Steinach sur les Rats : inversion sexuelle.

Le progrès véritable dans les problèmes de biologie générale consiste à transporter sur un terrain vraiment expérimental une controverse jusque-là purement spéculative. Si l'origine et la différenciation des caractères sexuels secondaires se dérobent et se déroberont sans doute toujours à cette orientation, parce qu'elles dépendent avant tout d'un facteur sur lequel nous n'avons aucune prise, à savoir une immense durée, - il n'en est pas de même du mécanisme actuel de leur réalisation dans l'individu. Leur corrélation avec les glandes génitales a été, en effet, récemment analysée par des procédés expérimentaux divers qui se rattachent à deux principaux : la castraíon, c est-à-dire la suppression des glandes génitales 
et leur transplantation. Nous allons examiner les principaux résultats ainsi acquis.

Quels sont les effets de la castration sur le reste de l'organisme et en particulier sur les caractères sexuels secondaires?

§. - La castration par sénilité. - Arrhénoïdie et Thélyidie. - Il y a d'abord, chez certains animaux au moins, unie sorte de castration physiolugique, que l'on appeler la castration par sénilité. A partir d'un certain àge, l'individu devient inapte à la reproduction. Toutefois, ce n'est pas une castration complète, car, si la fonction essentielle des glandes génitales ne peut plus s'exercer, les glandes restent encore en place et peuvent continuer à aroir une infl sence sur l'organisme. Les effets de la castration sénile sont loin d'ètre uniformes; mais chez d'assez nombreuses espèces de Mammifères et d'Oiseaux (les seuls groupes où ils aient pu. être suffisamment étudiés), ils consistent en une inversion plus ou moins marquée et plus ou moins fréquente des caractères sexuels secondaires. La femelle vieillie acquiert certains des caractères propres au mâle; plus rarement, le mâle vieux se rapproche du type de la femelle. Ces modifications ont été appelées, la première arrhénoïdie, la seconde thélyidie ${ }^{1}$.

Aristote mentionne déjà que, chez certains Oiseaux, les vieilles femelles tendent à prendre le chant du mâle et manifestent certains de ses instincts.

Les Faisans présentent ces phénomènes avec une assez grande netteté, et, comme ces animaux ont été beaucoup observés dans les faisanderies, la connaissance en est assez ancienne. On en trouve des descriptions nettes au xvin ${ }^{e}$ siècle. Is. Geoffroy Saint-Hilaire

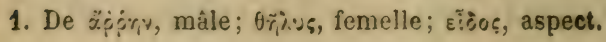


en a fait une excellente étude dans ses Essais de zoologie générale ${ }^{1}$. Il y décrit trois cas typiques, observés très complètement à la Ménagerie du Muséum (sur le faisan commun, le faisan argenté et le faisan à collier); de vieilles poules ont acquis graduellement le plumage du mâle. D'année en année, cette métamorphose devenait plus complète. L'une de ces femelles avait même des ergots comme les coqs; mais cet individu, ayant été moins suivi, Geoffroy ne peut affirmer que cette particularité ait apparu nettement avec l'arrhénoïdie. Le fait global est d'ailleurs connu des chasseurs, qui appellent coquards les faisanes ainsi modifiées.

Darwin a réuni tous les faits connus de ce genre, dans ses Variations des animaux et des plantes à l'état domestique et, plus récemment, Alex. Brandt a publié, a ce sujet, une étude d'ensemble sur les Oiseaux. L'arrhénoïdie est signalée chez la Poule domestique, le Faisan, le Paon, le Dindon, la Perdrix grise, le Coq de bruyère et des espèces roisines, le Pinson, le Bouvreuil, le Verdier, le Merle, l'Etourneau, le Coucou, le Combattant. le Canard domestique, etc... Au marché de Moscou, on en trouve assez fréquemment des exemples sur les Cioqs de bruyère expédiés de Sibérie. (Tetrao urogallus, T'. tetrix.)

L'arrhénoïdie ne se manifeste pas exclusivement sur des oiseaux vieux; on la constate aussi quelquefois sur des jeunes, mais ce sont alors des individus dont l'ovaire est anormal. Elle offre des degrés très divers; parfois, elle est unilatérale. Elle est toujours consécutive à la stérilité.

Chéz les Mammifères, on connait divers exemples d'arrhénö̈die : des bois poussent parfois sur la tête de vieilles biches; de rieilles juments acquièrent les

1. 2• partie, pp. 493-3̈14. 
canines du mâle. Chez la femme, après la ménopause et particulièrement chez celles qui ont étẻ stériles. on roit tréquemment pousser de la barbe.

Des taits plus nombreux seraient très probablement enregistrés si on pourait observer assez completement le vieillissement des animaux saurages. Donoi qu'il ế soit, l'arrhénö̈die apparait comme correlative d'une altération des ovaires ${ }^{4}$, par sénilité ou autrement.

5 2. - Castration parasitaire. - La castration se trouve encore réaliséc dans la nature d'une façon plus ou moins complete chez beaucoup d'animaux sous l'action des parasites. Le parasitisme cependant n'entraine pas nécessairement la castration. Il cst des parasites qui puliulent dans un organisme, sans aroir daction nıarquéc sur les glandes génitales; dautres, au contraire, détruisent ces glandes, soit directement, en se dérelop!rant aux dépens de leur substance, soit indirectement, à distance, par une altération des échanges ou, comme on dit, du métabolisme gènéral de l'organisme. Le parasite délourne. à son profit, les substances nutritives à l'aide desquelles la glande sexuelle derrait s'édifier.

Or, dans un certain nombre de cas, la castration parasitaire, directe ou indirecte; entraine des altérations des caractères sexuels secondaires, sur les-

1. Larrhénoildie a été rattachée à la théorie brièvement mentionnée plus haut (p. 92), d’aprés laquelle le mâle présenterait uné étape de l'évolution de l'espèce plus avancée que la femelle. La castration sénile libèrerait une partie de l'énergie qui était retenue par la vie fonct: anclle de l'oraire et qui pourrait être employée désormais à l'achèrement de l'évolution individuelle en réalisant le type malle. C'est là une idée intéressante, mais jordre purement spéculatif, comme toute la théorie à laquelle elle se rattache. 


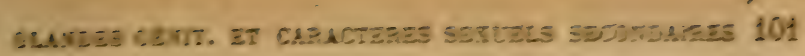
quelles on doit a Giand d'evoir attire Patte-ction des biologistes.

Le premier fait de cet ordre arait fide toutefits signale par J. Peres. Il avait montre que, ched certaines Abeilles, les Andrenes, paracitees par un Insecte
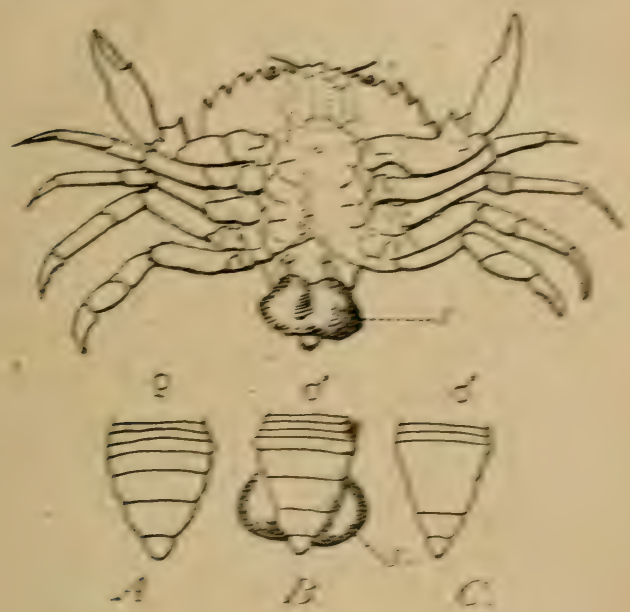

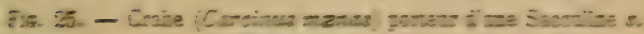

Vu vantoliemant

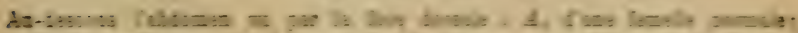

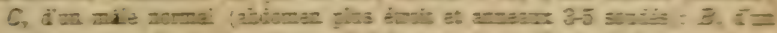

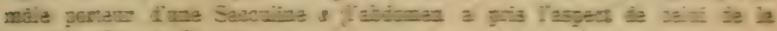
Semeile, - (Dagras Gand)

du geare Siylopr, les mules presentent plas ou moics raspect de femelles et inversement; il shgissit ici d'une castration parasitaire indirecte, le Stylopa etant tout is fait exterieur aux glandes gfnitales.

Sur divers Crabes parasites par des Orusacts Rtinocéphales, les Sacculines lig. 25, Giard a tbcourent des faits de meme ordin et duse extreme menteté 
l'étude détaillée en a été reprise plus récemment par G. Smith. Les deux sexes des Crabes diffèrent d'une facon très frappante par la forme de l'abdomen, plus large chez les femelles, plus étroit chez les mâles, où, de plus, plusieurs anneaux sont soudés entre eux (fig. 25, $C$ ). En outre, l'abdomen du mâle ne porte que les deux paires antérieures d'appendices transformées en stylets copulateurs, tandis que la femelle a cinq paires de pattes abdominales biramées et plumeuses auxquelles se fixent les paquets d'œufs composant la ponte. Or, chez les mâles parasités par une Sacculine, la forme de l'abdomen s'élargit, les anneaux sont moins intimement soudés ou mème complètement distincts, comme le montre la figure 25, B. Les pattes abdominales caractéristiques du sexe femelle peurent mème se dérelopper, comme l'a ru Smith sur des Inachus. Dans cette dernière espèce, les pinces du mâle prennent le type grêle correspondant au sexe femelle. Toutes cẹs transformations sont si marquées que d'anciens obserrateurs s'étaient mépris sur le sexe des mâles parasités et araient cru, à tort, que seules les femelles des Crabes étaient infestées par des Sacculines. La même erreur avait été commise aussi par un observateur éminent, Rathke, dans un autre cas, celui des Crevettes (Palæmon) parasitées yar un Epicaride Bopyrien, et c'est Giard qui l'a reconnue, guidé par les faits de castration parasitaire.

Les effets ne sont pas symétriques sur les deux sexes. Dans le cas des Crabes, la femelle est beaucoup moins modifiée que le mâle.

Les altérations des caractères sexuels secondaires et de la sexualité en général en rapport arec la castration parasitaire sont très répandues dans le règne animal et même chøz les Végétaux. Giard en a fait connaitre un grand nombre que je ne puis énumérer icı. Je citerai, à titre d'exemple, chez les 
plantes, le cas d'une Caryophỵllée très commune, Lychnis dioica, dont les sexes sont normalement séparés; elle est parfois parasitée par un Champignon du groupe des Ustilaginées et, dans ce cas, les pieds femelles forment des étamines où fructifie le parasite. Ici, le parasitisme, tout «n amenant la castration, rétablit l'hermaphrodisme morphologique.

G. Smith a eu l'occasion d'observer un fait, jusqu'i un certain point comparable au précédent, chez des Inachus sacculinés. Des individus mâles, qui s'étaient débarrassés de leur Sacculine, araient, acquis dans leur glande génitale des ovules rudimentaires. Le parasite, après aroir d'abord châtré son hòte et produit l'inversion de ses caractères sexuels secondaires, avait proroqué un rudiment d'hermaphrodisme; mais ce cas reste exceptionnel.

$\mathrm{Au}$ point de rue physiologique, ces actions des parasites ne peuvent se concevoir que par une altération du métabolisme de l'hòte. Sur des. Crabes (Inchus) sacculinés, Smith a constaté que la présence d'une Sacculine amène chez le màle une modification de la teneur du sang en substances grasses; elle y amène un taux égal à celui qu'on trouve chez les femelles. Elle semble donc bien faire du milieu intérieur du mâle celui d'une femelle.

Les effets de la castration parasitaire sont très variables suivant les types et, pour un type donné, suivant les individus. Il ne faut pas se les représenter simples et uniformes. On peut saisir au moins un des facteurs importants de la diversité des résultats ; c'est l'àge auquel l'hôte est atteint par le parasite. Si tous les Crabes sacculinés ne se montrent pas modifiés au mème degré, c'est évidemment en grande partie parce qu'ils n'ont pas été infectés au même âge. Mais il y a aussi, sans doute, d'autres éléments dans ces phénomènes qui sont très complexes. 
§ 3. - Castration expérimentale. - Arrivons main- . tenant à la castration proprement dite, c'est-à-dire à la suppression expérimentale des glandes génitales. Ses effets sur les animaux supérieurs - en particulier sur les animaux domestiques et aussi sur l'Hommeont été observés de longue date. Ils portent à la fois sur la forme et la structure des organes et sur la psychologie des individus.

La pratique de la castration chez les animaux domestiques repose sur cette constatation. Le mâle châtré est beaucoup plus maniable pour l'homme. Le cheral hongre, le bœuf, pour ne citer que ces deux exemples vulgaires, sont plus paisibles que l'étalon ou le taureau. L'altération générale des échanges dans l'organisme se rérèle, chez la plupart des castrats, entre autres caractères, par un envahissement de la graisse. Quant aux transformations morphologiques proprement dites, elles sont multiples et portent sur toutes les parties de l'organisme, en particulier sur le squelette. D'où une altération générale de la physionomie de l'animal. La tète et les cornes du bœuf sont très différentes de celles du taureau et rappellent la vache. Ne pouvant faire ici une revue détaillée 1 des faits, je m'en tiendrai à un petit nombre d'exemples.

Les Eunuques. - Le premier que j'étudierai est celui de l'Homme. La castration a été une pratique assez répandue autrefois. Elle était encore opérée au xvıri $^{e}$ siècle, en Italie, où elle portait, paraît-il, annuellement sur environ 4.000 enfants. Aujourd'hui, on ne la trouve plus qu'en Orient et en Asie. L'étude

1. On trouvera une revue très complète des faits relatifs à la zastration, et, d'une manière générale, aux divers problèmes concernant les caractères sexuels secondaires, dans Fortschrille ler naturwissenschaftlichen Forschung, t. 5, 1912. (KAMMERER, Ursprung der Geschlechtsunterscliede, 240 p..) 
précise des castrats, c'est-à-dire des eunuques, est pratiquement difficile à faire. Aussi n'a-t-on eu jusqu'assez récemment que des données fragmentaires. Dans ces dernières années, des recherches d'ensemble plus complètes ont été publiées, notamment par Matignon, sur les eunuques chinois, et par Tandler et Grosz, sur les Skopzis, secte russe qui pratique la castration et dont beaucoup de membres exercent, à Bucarest, la profession de cochers.

Les effets de la castration sur l'homme dépendent en premier lieu de l'âge auquel elle a été pratiquée; ils sont d'autant plus marqués que cet âge est plus précoce et qu'elle a été plus complète (sur certains sujets, en effet, les testicules seuls ont été enlevés; chez d'autres, le pénis a été sectionné). Les eunuques prennent une physionomie spéciale résultant de divers éléments. Chez beaucoup, il se fait un abondant dépôt de graisse au visage (en particulier aux paupières supérieures qui sont lourdes), dans la région mammaire, à l'abdomen, sur les crètes iliaques et aux fesses. Toutefois, à côté de ce type adipeux, il y a des eunuques maigres. Le teint est pâle et prend peu le hâle au soleil. Les formes générales du corps sont modifiées, surtout par les altẻrations dans la croissance du squelette, qui garde plus ou moins longtemps un caractère infantile. Le crâne est plus petit. Les épaules sont grêles; le bassin est large (sans avoir, comme on l'a dit parfois, la forme féminine). Les cartilages par lesquels s'allongent les os longs des membres pendant l'enfance ne s'ossifient que tardivement; il en résulte un allongement des membres relativement au torse ${ }^{1}$. Les cartilages du larynx ne se calcifient pas; le larynx garde ainsi une

1. L'envergure (distance d'une extrémité à l'autie des bras étendus horizontalement) chez les eunuques, est supérieure à 
structure infantile; la pomme d'Adain ne fait pas saillie comme chez l'homme normal; la mue de la voix ne s'opère qu'incomplètement ou pas du tout 2 . La pilosité est très modifiée. Tandis que les chereux sont abondants et tombent relativement moins que chez l'homme normal, le reste du système pileux reste à l'état infantile. Le visage est glabre, les aisselles et la région pubienne le sont plus ou moins. Les conduits génitaux et la prostate subissent (de mème que le pénis quand il est laissé) un arrèt de développement plus ou moins complet et restent sensiblement au stade qu'ils araient atteint au moment où la castration a été effectuée. Les instincts génitaux ne sont pas toujours entièrement abolis. Certaines glandes à sécrétions internes mortrent aussi des modifications: le thymus persiste plus longtemps que chez les sujets normaux. L'hypophyse est plus grande.

Ces quelques données, qui ne visent pas à être une description complète, montrent sur le cas de l'homme, où les repères sont le plus universellement appréciables, les transformations considérables et générales que produit la castration.

Ces diverses altérations du corps se retrourent à un degré plus ou moins étendu chez les individus qui présentent des malformations congénitales des testicules, ou chez qui ces organes ont été gravement lésés dans l'enfance par des maladies infectieuses. On peut appeler, arec Tandler et Grosz, ces sujets des Eunuchoides.

leur taille. Voici à ce sujet des nombres (en centimètres) cons, tatés par Tandler et Grosz chez quatre individus :

$\begin{array}{lllll}\text { Taille. . . . . } & 156 & 173 & -186 & 188 \\ \text { Envergure. . . } & 163 & 188 & 193 & 504\end{array}$

2. Tout le monde connait l'exemple des chantres de la Chapelle Sixtine. 
Sur les effets de la castration chez la femme, nous possédons des données beaucoup plus restreintes. Elle n'a été réalisable que par la chirurgie moderne et, par suite, elle se trouve exclue de toutes les pratiques ethniques. Chirurgicalement, elle n'est naturellement effectuée que pour des nécessités survenant seulement chez les adultes; elle n'entraîne par conséquent aucun trouble de croissance ni altération consécutive des proportions du corps adulte. Resteraient des sujets comparables aux eunuchoïdes, où la castration résulterait de malformations congénitales ou d'atrophies pathologiques au cours de l'enfance. Les faits connus à cet égard ne forment pas un ensenıble comparable à ce qu'offre le sexe mâle.

En somme, la castration chez l'homme entraine surtout la persistance des caractères infantiles, soit par arrêt de croissance, soit par le recul ou la suppression des phénomènes qui marquent la fin de la croissance, tels que l'ossification du squelette. Ce n'est qu'indirectement que certains de ces résultats produisent une ressemblance arec le trpe féminin.

La castration et les bors des Cerfs. - L'étude des faits relatifs à la castration chez les Mammifères ou les Oiseaux conduit à des conclúsions analogues. Je dirai quelques mots de ceux que présentent les Cerfs, où les bois constituent un caractère sexuel secondaire très frappant: les mâles seuls er possèdent et les renouvellent chaque année; la biche n'en a pas, sauf dans des cas exceptionnels ${ }^{1}$. Il résulte des documents réunis par Rörig que les biches porteuses de bois, et dont on a pu reconstituer l'histoire, araient des ovaires atrophiés ou malades, ou encore étaient plus ou moins

1. Ce dimorphisme ne s’étend pas à tous les Cervidés : ls Renne, par exemple, a des bois dans les deux sexes. 
hermaphrodites, ou enfin étaient de rieilles feme..es chez qui les bois avaient poussé tardivement, par un phénomène d'arrhénoïdie. Cependant il faut ajouter que le déreloppement des bois chez la biche, comme aussi diverses anomalies de ces formations chez le Cerf, peuvent survenir à la suite de traumatismes d'organes autres que les glandes génitales.

Quant à la castration chez le Cerf mâle, son retentissement sur les bois dépend de l'âge où elle est effectuée et de l'état des bois à ce moment. Pratiquée de bonne heure, avant la poussée des tubercules frontaux, elle empêche complètement la formation de la ramure. Si la castration n'est que partielle ou si elle a lieu au moment de la poussée, les bois restent faibles ou imparfaits. Si enfin elle est effectuée après la poussée terminée, les bois tombent au bout de quelques semaines, repoussent, mais sont définitifs, au lieu de tomber chaque année, comme cela a lieu normalement. L'atrophie des testicules entraine presque toujours la malformation connue sous le nom de bois en perruque. La forme générale du crâne est, elle aussi, altérée par la castration. Cet exemple montre une corrélation très nette entre la glande génitale et un caractère sexuel secondaire important; mais cette corrélation n'est pas absolument spécifique, puisque des traumatismes extragénitaux peuvent avoir un retentissement analogue.

Faits relatifs à d'autres Vertébrés. - En dehors des Hammifères, la castration a été pratiquée surtout sur la volaille. Pour des raisons anatomiques, elle ne peut être complète chez la poule. Chez le Coq, elle produit les transformations qui caractérisent le chapon et dont l'intensité est variable, parce qu'elle n'est pas toujours totale. Nous verrons en effet plus loin que s'il reste des fragments de testicule, même en dehors de leur posi- 
tion normale, les effets de la castration sont en grande partie annihilés. Le chapon a perdu le chant et les instincts belliqueux du coq; par contre, il couve; la crète est réduite, pàle et flasque. Les éperons subsistent et le plumage r'est pas altéré.

De nombreuses expériences ont été faites également sur les Grenouilles. Chez les mâles, le doigt interne des membres antérieurs présente une callosité glandulaire (fig. $26 \mathrm{~A}$ ) qui manque chez les femelles.

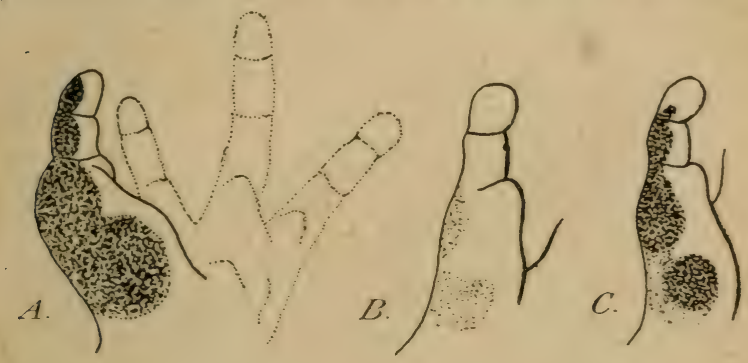

Fig. 26. $-A$, patte antérieure de citenouille mảe normale, montrant la tulútosité du doigt interne et le grand developpement de glandes cutanées (ombrées'; $B$, doigt interne de la patte antérieure d'une Grenouille mâle chà̀trée (atrophie de la tubérosité et des glanłes); $C$, Même doigt pris sur une Grenouille mile chàtrée, mais à qui on tit ensuite, lans la peau, des injections de substance testiculaire (réapparition de la tubérosité et des glandes). (D’aprẻs Meisenheimer.)

Or, si l'on châtre un màle, la callosité s'atrophie $(B)$. Nous verrons plus loin qu'elle reparaît $(C)$ si, après castration, on greffe sous la peau un testicule ou si l'on fait au castrat des injections de substances testiculaires.

De l'ensemble des observations et expériences pratiquées surles Vertébrés, on peut corc'ure que les glandes génitales ont une action considérable et complexe sur la croissance et la transformation de l'ensemble du corps, influence que la castration met en évidence. 
Ce ne sont donc pas spécifiquement les caractères sexuels secondaires qui sont atteints; ils le sont comme le reste de l'organisme, mais au fond la distinction n'a pas une très grande importance, car ces caractères. nous le répétons, n'ont à aucun degrré, une existence autonome. En fait, cependant, la ressemSlance du mâle arec la femelle se troure augmentée, mais cela tient à ce qu'il y a, en réalité, persistance plus accentuée des caractères infantiles et que la femelle normale conserve ceux-ci, plus que le mâle.

La castration chez les Insectes. - Résultats négatifs des expériences. - Ce résultat global, qui s'applique, semble-t-il, à tous les Vertébrés, ne peut ètre absolument rénéralisé. En effet, de nombrenses expériences, faites dans ces dernières années sur les Insectes, ont montré, d'une façon très concordante, des résultats opposés en apparence, au moins. Des obserrateurs, Oudemans, Kellogg, Meisenheimer, Kopec, ont supprimé (en les sectionnant on en les brùlant au galrano-cautère) les glandes génitales de très jeunes larres, sans qu'il soit résulté aucune altération nette des caractères sexuels secondaires. La taille, la forme, la colorration, les instincts sexuels des adultes restent ce qu'ils eussent été, si l'on n'avait pratiqué aucune intervention expérimentale. On a opéré surtout sur les Chenilles, principalement sur celles des Bombyciens, et en particulier sur une espèce commune, oủ le dimorphisme sexuel est très accenlué, Lymantria dispar ${ }^{1}$, ce qu'exprime d'ailleurs le nom spécifique. La différenciation des caractères sexuels secondaires parait donc ici indépendante de toute action des glandes génitales. Mais il ne faudrait 
pas formuler cette conclusion d'une façon trop catégorique. Nous arons $\mathrm{vu}^{1}$, en effet, que, chez les Insectes, le tissu germinal s'individualise des les débuts du développement de l'œuf, à une période bien antérieure aux stades les plus jeunes sur lesquels ont porté les expériences précédentes. D'autre part, on sait maintenant que les élauches des organes de l'insecte parfait, du Papillon dans le cas actuel. se forment aussi d'une façon très précoce. Il est donc possible que le moment où on a pu interrenir expérimentalement soit déjà trop tardif et que les cellules génitales avaient exercé déjà une action définitive.

\section{4. - La transplantation des glandes génitales. -}

La castration troure un complément expérimental dans la transplantation des glandes génitales. Celle-ci peut ètre pratiquée soit seule, soit surtout après castration partielle ou totale. On peut la faire, en greffant en une position différente de la normale, les glandes de l'individu mème auquel elles appartenaient (c'est ce qu'on appelle la transplantation autoplastique), ou en les insérant dans un autre individu de la même espèce (transplantation hétéroplastique), ou enfin dans un individu d'espèce différente. Plusieurs expériences de ce genre sont déjà fort anciennes, mais c'est surtout dans ces dernières années, qu'on en a pratiqué sur les Vertébrés (Mammifères, Oiseaux, Batraciens) et les Insectes, pour étudier les problèmes de la sexualité. Je me borne ici à quelques brères indications à ce sujet. D'une manière générale, chez les Vertébrés, les effets conatatés à la suite de la castration totale sont diminuès, ou même complètement supprimés par la transplantation.

La pratique courante de la castration chez le coq a

-1. P. 15. 
: amené depuis fort longtemps à des expériences de transplantation sur cet animal. Dès 1849, Berthold a montré d'une facon très précise que la transformation en chapon n'est réalisée que si l'ablation des testicules est complète; il arrive fréquemment que des fragments de ces organes se greffent en des points divers du péritoine, où ils évoluent; dans ces conditions, l'animal garde plus ou moins les caractères du mâle normal. Berthold a fait expérimentalement sur le Coq la greffe autoplastique et hétéroplastique, et plusieurs expérimentateurs ont depuis répété et varié ces expériences; il s'en dégage que la transplantation des testicules efface plus ou moins les effets de la castration.

Chez la Grenouille rousse (Rana temporaria), Nussbaum a montré, et le fait a été confirmé par divers auteurs, que si l'on châtre un mâle, les callosités des pouces antérieurs, particulièrement développées à l'époque de la reproduction, régressent, mais qu'elles se maintiennent au contraire si, après la castration, on a greffé, par exemple sous la peau, dans le sac lymphathique dorsal, un testicule ou un fragment de cet organe.

Tout récemment, Steinach a transplanté, dans des Rats châtrés à l'àge de quatre semaines, destesticules de leurs congénères. La greffe réussit et les individus ainsi traités acquirent le lype et les instincts du mâle normal, et non ceux du castrat.

Cette influence des glandes génitales transplantées est attestée par des expériences sur diverses espèces de Mammifères. Chez la femme même, la littérature médicale renferme quelques exemples de greffes d'ovaires à la suite desquelles ont été rétablies des fonctions absentes, telles que la menstruation. D'une façon générale, après transplantation des ovaires chez la femelle jeune, les roies grénitales se dérelop- 
dent, tandis qu'elles restent rudimentaires sur la femelle châtrée.

Bien intéressantes sont les expériences les plus récentes (1911) de Steinach, sur la transplantation croisée des glandes et conduits génitaux d'un sexe à l'autre. Il opéra sur des Rats. De jeunes rats mâles, châtrés, auxquels on a greffé des ovaires, n'acquièrent pas les caractères mâles. Le pénis, en particulier, reste rudimentaire comme un clitoris; les ovaires implantés ont exercé une action inhibitrice sur les caractères sexuels mâles. Mais si, en mème temps que les ovaires, on implante à ces jeunes mâles châtrés l'utérus et les trompes, tout l'appareil femelle greffé se déreloppe et le sujet mâle ainsi traité acquiert une série de caractères sexuels secondaires femelles. II est littẻralement féminisé. Il a, de la femelle, le poil et l'adiposité. Son appareil mammaire est à l'état que l'on trouve chez la femelle vierge. Les instincts mèmes, d'après Steinach, sont plus ou moins inversés; il a, dans le port, des réflexes féminins et donne à ses congénères l'illusion d'une femelle véritable.

Ces diverses expériences montrent, comme celies sur la castration, que la glande génitale exerce chez les Vertébrés une action très puissante sur l'organisme entier et en particulier sur les caractères sexuels secondaires.

Les expériences de même ordre faites sur les Insectes, notamment celles de Kiopec et de Meisenheimer, ont fourni au contraire des résultats négatifș, comme déjà celles de la castration. La transplantation croisée des glandes génitales dans de très jeunes Chenilles de Lymantria dispar n'empêche pas le papillon de conserver complètement les caractères de son sexe primitif. 


\section{CHAPITRE X}

\section{CARACTĖRES SEXUELS SECONDAIRES ET SËCRÉTIONS INTERNES (HORMONES)}

Les sécrétions internes ou hormones. - La fonction glycogénique du foie. - Généralité des actions hormoniques. - Appareils à sécrétions internes spécialisées thyroïde, capsules surrénales .

- Progrès récents de nos connaissances sur ces actions. Action régulatrice générale des hormones dans l'organisme. - Coordination nerveuse et régulation chimique.

L'appareil génital agit sur l'organisme par des sécrétions internes. - Interprétation des expériences sur la castration et la transplantation des glandes génitales. - Travaux de Bouin et Ancel. - Glande interstitielle du testicule. - Corps jaune de l'ovaire. - Etérus et glande mammaire.

Valeur des différences entre les faits observés chez les Arthropodes et chez les Vertébrés.

\$1. - Les sécrétions internes ou hormones. Envisagé uniquement au point de vue morphologique, l'erısemble des faits exposés au chapitre précédentest assez discordant. La physiologie commence à y projeter une assez vire lumière en en expliquant le mécanisme, et, par là, en faisant conceroir la possibilité des différences constatées. Une notion lomine, en effet, aujourd'hui, la manifestation des caractères sexuels secondaires, au moins chez les Tertébrés; c'est celle du rôle que jouent les sécrétions uternes ou, comme on dit aujourd'hui, les hormones 1

1. De ópráx $\omega$, j’éveille. 
suivant le terme proposé par Bayliss et Starling.

$\mathrm{La}$ glande génitale agit sur le reste de l'organisme par des sécrétıons internes; on a pu, par des expé riences précises, en donner la preuve. Ce n'est pas là, du reste, un mécanisme spécial aux caractères sexuels secondaires. Ils ne constituent en rien une catégorie spéciale dans l'organisme et la doctrine des sécrétions internes montre, au contraire, combien la conception de cez caractères particuliers, pratiquement nécessaire pour la description et l'analyse des faits, est purement subjective, la seule réalité étant l'organisme. tout entier.

La notion de sécrétion interne, si elle s'est beaucoup déreloppée dans ces dernières années, n'est pas nourelle. C'est à Claude Bernard que nous la derons; la fonction glycogénique du foie en est, en effet, un exemple typique et d'une importance capitale. Les glandes nous ont apparu d'abord comme des appareils élaborant un produit, la sécrétion, qu'elles déversent hor's du milieu interne de l'organisme : à l'extérieur, comme la sueur, le sébum ou le sperme, ou dans la cavité intestinale qui, à parler strictement, est encore une partie de l'espace extérieur. Mais elles peurent aussi élaborer d'autres produits qui passeront dans le sang, et ce sont ces produits que nous appellerons des sécrétions internes.

Le foie déverse la bile dans l'intestin, c'est sa sécrétion externe; il élabore le glycogène dans ses cellules et, aux dépens de ce glycogène, forme du sucre qu'il déverse dans le sang, de façon que la teneur en sucre de ce sang soit constante, c'est sa sécrétion interne. Ici, apparait déjà le rôle capital de ces sécrétions; elles sont essentiellement régulatrices. Elles maintiennent laconstance du milieu soit directement, comme dans le cas précédent, soit indirectement, en provoquant ailleurs des réactions cam- 
pensatrices. A cóté du système nerveux, qui est l'appareil principal de coordination entre les parties de l'organisme, elles représentent, elles aussi, un mécanisme assurant la stabilité du tout et l'harmonie des parties.

A vrai dire, la fonction de sécrétion interne n'est pas limitée à certaines glandes. Elle est universelle. Toute cellule de l'organisme, par son fonctionnement vital même, échange arec le milieu où elle baigne des substances chimiques; elle y dérerse des produits de son activité, dont aucun n'est indifférent à ce milieu, c'est-à-dire à l'organisme. Et pour en prendre un exemple saisissant, le résultat des combustions organiques, l'ácide carbonique, est un produit de toutes les cellules, une sécrétion interne commune à toutes, qui se dérerse dans le sang. Or, la teneur du sang en acide carbonique est un facteur d'importance capitale, puisque c'est de lui que dépend le fonctionnement normal des centres nerveux respiratoires. Sans doute, entre la production d'acide carbonique par les direrses cellules et le fonctionnement des centres respiratoires, il y a de puissants appareils régulateurs, tels que le poumon, mais, en principe, toute cellule, en déversant de l'acide carbonique et aussi d'autres substances, joue un rôle de sécrétion interne.

Toutefois, par l'application universelle de la division du trạail, ce rôle général est spécialisé pour certaines substances, dans des appareils localisés. Le foie sécrète ainsi du sucre. Le pancréas, à côté de la sécrétion externe, le suc pancréatique, a aussi un rôle important de sécrétion interne ${ }^{1}$. Il existe surtout une catégorie d'organes, dont la fonction est

1. Voir E. HÉDox. Les raprots du panci is avec le diabète. Biologica, t. 3, 1913, p. 40. 
restée énigmatique jusqu'à une période récente; ils ont une texture plus ou moins glandulaire, mais ne présentent pas de voies efférentes pour leurs produits, et par contre, ils sont très très vascularisés. On les a appelés jadis glandes vasculaires sanguines, et on a conclu qu'elles rejetaient dans le sang un produit de leur activité, une sécrétion interne.

Dans ces dernières années, la nature de ces sécrétions, leur rôle propre s'est beaucoup précisé. La glande thyroïde, le thymus, les capsules surrénales ont été particulièrement étudiés. Pour ces dernières surtout, l'un des produits de leur activité, l'adrénaline, a pu être isolé, étudié chimiquement de façon complète, et sa connaissance a eu un retentissement considérable sur des questions multiples de physioJogie et de pathologie. Nous sommes moins avancés pour d'autres appareils, tels que l'hypophyse, placée sous le cerveau, ou l'épiphyse. A côté de ces organes, anatomiquement connus depuis longtemps, il en est d'autres, permanents ou temporaires, dont le rôle se révèle et se précise peu à peu.

L'importance de certaines glandes à sécrétions internes, comme l'appareil thyroïdien ou les capsules surrénales, ressort, au reste, suffisamment du fait brutal que l'ablation d'un de ces appareils, si petits cependant, entraîne rapidement et fatalement la mort. Les substances qu'ils élaborent sont essentielles au fonctionnement del'organisme ${ }^{1}$. Tout fait soupconner leur étroite solidarité et, sans que cette notion soit encore parfaitement précisée, on devine dans ces organes les agents principaux de l'harmonie des par-

1. L'organe de Bidder des Crapauds mâles qui, morphologiquement, constitue une ébauche d'hermaphrodisme (cf. p. 53), joue évidemment le rôle d'une glande à sécrétion interne annexée au testicule, et sa fonction est essentielle puisque son ablation est mortelle. 
ties dans l'organisation totale. L'équilibre, qui donne si fortement l'impression d'une finalité dans les manifestations de la vie, n'est sans dya \& que le résultat a posteriori des actions réciproques des sécrétions internes sur les direrses parties de l'organisme. Il y a tout au moins, en elles, un mécanisme qui nous aide beaucoup à concevoir la possibilité de cette harmonie automatique.

\$2. - Les hormones et la sexualitè. - Elles jouent un rôle considérable dans les phénomènes de la sexualité, et il faut y chercher l'explication principale de ceux que nous arons précédemment étudiés. La glande génitale agit sur le reste de l'crganisme par des sécrétions internes. Celles-ci sont indispensables à la réalisation typique des caractères sexuels secondaires. C'est la suppression des sécrétions internes de la glande génitale qui produit les déformations caractéristiques du castrat. La castration incomplète ou la transplantation laissent subsister une certaine quantité de tissu génital avec les sécrétions internes et corrélativement, ainsi que nous l'avons vu, l'organisme ne subit pas les stigmates de la castration totale.

Il s'agit bien d'une action chimique. Deux ordres d'arguments le prouvent. En premier lieu, beaucoup des effets de la castration peuvent être supprimés par des injections de substance testiculaire broyée. Par des injections de cette nature, divers observateurs, et notamment Pézard, obtiennent, chez le Coq châtré, la turgescence des crêtes et la persistance ou la réapparition des instincts du mâle. Steinach, en injectant dans les sacs lymphatiques, à des Grenouilles châtrées, de la substance de testicule broyée, obtient le développement de la callosité des pouces et le réflexe de l'embrassement de la femelle par le 
mâle. On pourrait citer d'autres exemples analogues.

L'initiateur de ce mourement expérimental a été Brown-Séquard, quand en 1889, il annonça les effets stimulants d'extraits testiculaires sur l'homme et spécialement sur le vieillard. De ces expériences, accueillies d'abord avec scepticisme et mème avec hilarité, est sorti le mourement qui a conduit à l'opothérapie. L'ingestion ou linjection de la substance de glandes rasculaires sanguines, telles que la thyroïde, supplée à une insuffisance plus ou moins grande du fonctionnement de ces glandes.

Comme dans tous les problèmes analogues, l'explication des faits par une sécrétion interne, c'est-àdire par une action chimique à distance, a eu à triompher de l'interprétation pardes réflexes nerveux, ayant leur origine dans les glandes génitales et passant par le système nerveux central. La méthode générale, pour répondre à cette objection, et, en mème temps, le second ordre d'arguments est de montrer que les effets subsistent quand l'appareil, siègre de la sécrétion présumée, est isolé de toutes ses connexions nerveuses arec le reste de l'organisme.

Les expériences de transplantation se prètent particulièrement, à ce genre de démonstration. Je citerai ainsi une récente expérience de Harms. Il transplante sur le dos d'une Grenouille mâle, normale, la peau du pouce d'un mâle châtré au printemps précédent et dont la callosité s'est atrophiée. Au bout de quelques semaines de séjour sur le dos de ce nouvel individu, la peau ainsi greffée se tuméfie en une callosité glandulaire. Ainsi, en dehors des connexions nerveuses normales, et mème rraisemblablement de toute connexion nerveuse, la callosité se développe; on ne peut attribuer le phénomène qu'à l'action chimique exercée sur la peau de pouce greffée, par le sang de 
?a Grenouille porte-greffe, et, vu les résultats des expériences de castration ${ }^{1}$, c'est à une action chimique, émanée des testicules qu'il faut la rapporter.

Est-ce à dire que les actions chimiques produites par les hormones aient toutes un mécanisme simple et immédiat? Il n'en est rien. Il y a une solidarité entre les diverses sécrétions internes, comme je le disais au début de ce chapitre. Les hormones génitales doivent influer sur la production des autres et réciproquement. C'est ce qu'on commence à entreroir en particulier pour l'hypophyse. Des constatations anatomiques montrent que cet organe est hypertrophié chez les eunuques; on sait d'autre part, qu'il montre constamment des altérations dans les cas d'acromégalie et de gigantisme; les déformations de cet ordre, que présentent précisément les eunuques, sont vraisemblablement en rapport immédiat avec des altérations de l'hypophyse, qui résultent elles-mèmes de la castration. La clinique a enregistré d'ailleurs la solidarité de l'appareil génital et de l'hypophyse. Par le traitement opothérapique, c'est-à-dire par l'ingestion de substance hypophysaire, on a tait disparaitre des troubles génitaux et des altérations de croissance relevant des glandes génitales.

Les hormones génitales ne doivent donc pas être concues comme des substances isolées du reste de l'organisme.

Il serait non moins excessif de nier tout rôle important du système nerveux. Le point de départ n'est pas une excitation nerveuse ayant son siège dans la glande génitale. Mais l'hormone n'agit pas nécessairement d'une manière direete. Il se peut (et pour bien des phénomènes, il semble qu'il en soit

1. Cf. p. 109. 
CARACTÉRES SEXUELS SECONDAIRES ET HORMONES 1:1

ainsi) qu'elle agisse sur le système nerveux central et que ce soit celui-ci qui, par la voie centrifuge, modifie les organes périphériques. L'action de l'hormone serait donc indirecte et se ferait par l'intermédiaire du système nerveux. Les hormones génitales érotiseraient les centres nerveux, suivant l'expression de Steinach. L'emirassement de la femelle par le mâle, chez la Grenouille, à l'époque de la reproduction, est déterminé par un réflexe qui, d'après cet auteur, serait, en temps normal, empêché par des centres nerveux inhibiteurs. Une hormone testiculaire, produite à l'époque du rut, affaiblirait ou supprimerait l'inhibition et permettrait ainsi au réflexe de se produire. La transplantation de testicules sur des Rats châtrés jeunes 'suffit à maintenir sur eux, à l'état adulte, les instincts sexuels qu'a perdus le castrat. Le tissu testiculaire transplanté sécréterait des hormones qui érotiseraient les centres nerveux.

L'action hormonique des glandes génitales et l'importance de ce mécanisme chimique dans la manifestation des caractères sexuels secondaires, au moins chez les Vertébrés, résulte donc d'un ensemble considérable de faits et vient prendre place, d'une facon très satisfaisante pour l'esprit, dans un cadre physiologique d'une grande ampleur. Mais les recherches récentes ont permis de préciser encore ces notions et en mème temps elles font concevoir une possibilité pour expliquer des contradictions, telles que celles que nous arons constatées, au chapitre précédent entre les Arthropodes et les Vertébrés.

La glande interstilielle du testicule chez les Vertibrés.-Les actions hormoniques génitales essentielles, en effet, n'ont pas nécessairement leur siège dans le tissu germinal proprement dit, mais peuvent l'avoir dans les éléments cellulaires interposés au milieu de 
celui-ci, ce qu'on appelle le tissu interstitiel ou la glande interstitielle. Cela résulte de nombreux travaux récents, parmi lesquels ceux de Bouin et Ancel ont une importance et une élégance toute particulières.

Nous arons distingué ${ }^{1}$, dans un testicule de Vertébré, trois catégories de cellules dans les tubes séminaux proprement dits : $1^{\circ}$ la lignée séminale qui aboutit à des spermatozoïdes; $2^{\circ}$ les éléments de Sertoli qui paraissent jouer surtout, vis-à-ris de la lignée séminale, un rôle nourricier; $3^{\circ}$ entre les tubes séminaux et formant la charpente du testicule, le tissu interstitiel; il renferme des cellules plus ou moins différenciées, élaborant des produits variés, tels que de la graisse, des pigments et vraisemblablement aussi des produits solubles.

On possède aujourd'hui divers moyens d'analyser ce complexe. L'action convenablement ménarrée des rayons $\mathrm{X}$ détruit la lignée séminale, sans altérer les deux autres catégories. La ligature expérimentale des canaux déférents produit, à la longue, le mème résultat; il en est de même de leur obturation consécutive à des infections inflammatoires des roies génitales. D'autre part, certaines malformations aboutissent au mème résultat. Il en est ainsi nolamment, quand les testicules ne descendent pas dans le scrotum, comme il est normal, mais restent internes. On dit alors que l'animal est cryptorchide. Or, la cryptorchidie s'accompagne généralement de stérilité et l'étude histologique de ces testicules, dits encore ectopiques, montre que les tubes séminaux n'y présentent que les éléments de Sertoli, les lignées séminales ayant avorté. En général, le tissu interstitiel y est conservé, quelquefois cependant il y est aussi atrophié : nous pourrons, avec Bouin et Ancel, distin-

1. Cf. p. 21. 
guer des cryptorchides de première et de seconde catégorie, suivant que le tissu interstitiel sera conservé ou atrophié.

Or, les cryptorchides de première catégorie, tout en étant stériles, conservent l'ardeur génésique, l'ensemble des instincts génitaux et même les formes normales, alors que ceux de seconde catégorié ont le psychisme et les formes du castrat. Si l'on fait à des castrats des injections d'extrait de testicules cryptorchides de première catégorie, on réveille chez eux les manifestations génésiques abolies. En injectant à des Coqs châtrés des extraits de testicules cryptorchides de Porc, Pézard ranime chez eux l'ardeur combattive, fait reparaitre le chant caractéristique, ramène la rigidité de la crète, tous effets qui disparaissent avec la cessation des injections. Les extraits de testicules cryptorchides de seconde catégorie, où le tissu interstitiel est atrophié, n'ont aucune de ces vertus.

En supprimant un des testicules sur un lapin et en ligaturant l'autre, Bouin et Ancel ont constaté dans celui-ci l'atrophie des lignées séminales, mais en mème temps une hypertrophie compensatrice du tissu interstitiel et, en même temps, étaient conserrés les instincts génésiques et la morphologie normale.

Toute une série de faits et d'expériences coordonnées, que je ne puis détäller ici, viennent donc montrer que les actions hormoniques émanant de la glande génitale, chez les Vertébrés supérieurs tout au moins, proviennent, non des cellules sexuelles proprement dites, mais du tissu interstitiel. La glande génitale renferme une glande interstitielle à sécrétion interne.

Le corps jaune de l'ovaire. - Il est extrèmement intéressant de rapprocher des données précédentes 
les résultats obtenus par divers auteurs et en particulier par Bouin et Ancel, dans un domaine qui se rattache étroitement à la sexualité, telle que nous l'arons envisagée ici; je veux parler du fonctionnement de l'ovaire et des modifications de l'organisme maternel pendant le développement de l'embryon, chez les Mammitères.

Yous avons dit ${ }^{1}$ qu'à l'ovule, dans l'ovaire de ces animaux, est adjoint un nombre considérable de cellules formant le follicule de de Graaf, et que ce follicule reste en place quand l'ovule même s'est détaché et est tombé dans la trompe, pour se rendre ensuite dans l'utérus. Le follicule se transforme alors en une masse cicatricielle, située à la surface de l'ovairé et appelée, en raison de sa couleur, le corps jaune. Celui-ci érolue très différemment, suivant que l'ovule est fécondé, se greffe sur l'utérus et se développe en un embryon, ou que, non fécondé, il est simplement expulsé au dehors. Dans le second. cas, le corps jaune se résorbe rapidement en quelques jours; dans le premier, au contraire, il persiste longtemps, pendant une bonne part de la gravidité, et présente une phase de développement suivie d'une régression progressive. Le corps jaune n'est donc pas une cicatrice pure et simple, mais bien un organe temporaire, dont le sort parait lié à celui de l'orule qui en prorient.

Il ressort des recherches récentes, et surtout de celles de Bouin et Ancel, que le corps jaune est une glande à sécrétion interne, dont les hormones déterminent des phénomènes très importants, à la fois dans l'utérus et dans la glande mammaire.

C'est en effet sous l'influence du corps jaune que s'effectuent les modifications de la paroi utérine, grâce auxquelles l'œuf pourra se greffer sur elle, et

1. Cf. p. 18 et fig. $T$. 
qui, chez certaines espèces, amènent la menstruation, Celle-ci en effet n'est pas produite par des réflexes nerreux partant de l'ovaire, car elle subsiste après qu'on a isolé l'ovaire. en coupant toutes ses connexions nerveuses; de mème, on a pu la faire réapparaitre, sur un sujet où elle était abolie, en transplantant un ovaire dans une position anormale.

Chez la lapine, les orules ne se détachent pas spontanément de l'oraire, mais seulement sous l'influence de réflexes proroqués par le coït ${ }^{1}$. Bouin et Ancel accouplent une jeune lapine vierge, présentant les premières chaleurs, arec un màle dont les canaux déférents ont été ligaturés plusieurs mois arant et qui par suite est parfaitement stérile, mais cependant ardent. Le coït proroquera donc l'orulation, mais ne sera pas fécondant et par suite n'amènera aucun des phénomènes accompagnant la fécondation des orules ou prourant résulter de la présence des spermatozoïdes. La formation des corps jaunes consécutive à l'orulation sera donc la seule modification surrenant dans l'appareil sexuel de la lapine. Or, les observateurs ont constaté, dans ces conditions, en sacrifiant des femelles ainsi traitées à des intervalles dirers, que l'utérus subit toutes les transformations préparant normalement la greffe des embryons.

On obtient des résultats parallèles, en effectuant expérimentalement la rupture des follicules de Graaf mûrs, chez une lapine vierge. Si enfin on détruit (par exemple par des cautérisations au galrano-cautère) les corps jaunes formés, aux différentes étapes de ces modifications de la paroi utérine, on roit celles-ci $\therefore$ arrèter à la suite de cette destruction. C'est donc le corps jaune de l'ovaire, qui détermine les trans-

1. Il en est de meme chez la cobaye et la chatte; au contraire l'orulation est spontanée chez la femme, les singes, la chienne, la jument, la truie, la vache, etc. 
formations de la paroi utérine préalables à la fixation de l'œuf fécondé. Et il s'agit bien d'actions hormoniques, non d'actions réflexes, car on obtiendrait la mème série de résultats, les ovaires étant coupés de toutes leurs connexions nerveuses.

C'est aussi le corps jaune qui, par des hormones, provoque les transformations de la glande mammaire au début de la gravidité. A ce moment en effet, cette glande est le siège d'une prolifération cellulaire active. Pendant la seconde moitié de la gravidité, il n'en sera plus de même; les cellules ne se multiplieront plus. mais elles commenceront à sécréter du lait, ce qu'elles ne faisaient pas antérieurement. Il y a ainsi deux phases bien distinctes dans l'érolution de la glande mammaire pendant la gestation. Le corps, jaune, par roie hormonique, est l'agent déterminant de la première ${ }^{1}$. En effet, dans les expériences sur la lapine relatées plus haut, où le corps jaune détermine le début des transformations de l'utérus, on roit se produire, en mème temps, la prolifération de la glande mammaire, et celle-ci s'arrête, comme le phénomène utérin, si on détruit prématurément le corps jaune. Il ne peut s'agir d'une action hormonique provenant du reste de l'ovaire, ni de l'utérus, ni du placenta, ni de l'embryon; car en injectant la substance de ces divers organes à une femelle, on ne provoque nullement les transformations de la glande - mammaire.

Le corps jaune apparaît donc, de par l'expérimentation méthodique, comme une glande à sécrétion

1. On a longtemps attribué ces transformations de la glande mammaire à des mécanismes nerveux réflexes, dont on plaçait le point de départ dans l'utérus ou dans l'ovaire. Mais ils subsistent quand on isole la glande mammaire de toutes ses connexions nerveuses; par exemple, quand on la greffe sur le pavillon de l'oreille. 
interne temporaire, déterminant à distance, par roie hormonique, les premières transformations de l'utérus et de la glande mammaire dans la gravidité.

Utérus et glande mammaire. - Par contre, il est impuissant à provoquer les phénomènes sécrétoires dont la glande mammaire est le siège à la fin de celle-ci, et, d'ailleurs, c'est au moment où ils vont se produire que, physiologiquement, le corps jaune se résorbe. Bouin et Ancel riennent de montrer, par des expériences ingénieuses que je ne détaillerai pas, que cette seconde phase de l'activité de la glande mammaire est sous la dépendance d'hormones émanant de cellules disséminées à trarers le placenta, et constituant temporairement, au moment où ra commencer la secrétion mammaire, une glande à sécrétion interne qu'ils ont appelée glande myométriale endocrine.

\$3. - Conclusions générales. - Ainsi, tout le cortège des modifications que présente l'utérus ou la glande mammaire aux direrses phases de la gestation chez les Mammifères, ce mécanisme, si admirablement coordonné pour assurer la vie de l'embryon, depuis l'état d'œuf jusqu'après la naissance. et si propre à éreiller en nous l'idée diune merreilleuse finalité et diune Providence préroyante: peut être réalisé en dehors de l'embryon et est ramené à une série de phénomènes physico-chimiques. Sans doute ils se commandent et se succèdent dans un ordre et avec un rythme appropriés. Mais ils ne sont pas plus merveilleux que n'importe lequel des processus vitaux, que, par exemple, la régulation automatique de la teneur du sang en acide carbonique, gràce à laquelle peurent fonctionner les centres respiratoires. Il y a là, dans les rapports du 
milieu et de cette unité que constitue l'ensemble de l'organisme, une série de conditions et d'équilibres physico-chimiques, dont chacun conditionne le suirant, le rend possible et nécessaire, sous peine que tout s'arrête. C'est, au reste, l'essence même de l'hérédité et de tous les phénomènes successifs constituant le déreloppement des organismes.

On voit combien, dès à présent, la considération des hormones jefte de lumière sur le mécanisme de la manifestation d'un grand nombre de caractères sexuels secondaires et aussi des phénomènes psychiques de la sexualité. Chez les Vertébrés, il est curieux de constater que ce n'est pas le tissu germinal lui-même qui exerce l'action décisive, mais un tissu extragénital incorporé à la glande sexuelle, et formant lui-même un appareil glandulaire hormonique, la glande interstitielle, comme l'appellent Bouin et Ancel, la glande de la puberté, comme Steinach l'a nommẻe d'une façon très expressive.

Il y a donc une dissociation plus ou moins complète de la fonction génitale proprement dite et de l'action morphogène sur les diverses parties de l'organisme.

Cela peut diminuer la contradiction que présentent, guant à l“influence de l'appareil génital sur les caractères sexuels secondaires et le psychisme de la sexualité, des embranchements tels que les Arthropodes et les Vertébrés. Ces deux groupes sont deux phylums tout à fait indépendants du règne animal et dont la structure histologique est différente pour tous les systèmes d'organes, en particulier pour les glandes rénitales. Les résultats négatifs constatés plus haut, chez les Arthropodes, à la suite de l'ablation de ces glandes ou de leur transplantation, tiennent peutètre simplement à ce que, chez ces animaux, l'appareil équivalant à la glande de la puberté, ou bien 
n'existe pas, ou tout au moins n'est pas incorporé à la glande génitale. En tout cas, le fait que le tissu germinal n'est pas nécessairement le siège de l'agent morphogène des caractères sexuels secondaires lève la contradiction, qu'on pourait trouver catégorique, entre les deux groupes.

Le gynandromorphisme constitue évidemment un phénomène difficile à concilier arec les explications hormoniques précédentes. Giard y royait mème un obstacle insurmontable à admettre celles-ci. Une substance chimique dissoute doit éridemment agir sur la totalité de l'organisme et on s'explique mal, par elle, la répartition en mosaïque des caractères mâles et femelles, sur un même individu. Mais le gynandromorphisme est au moins extrèmement rare chez les Vertébrés et n'a pu y ètre suffisamment étudié. Quant aux Arthropodes, où il est plus fréquent, nous pourons dire provisoirement que les caractères sexuels secondaires $y$ échappent aux actions hormoniques ou que, du moins, nous ne connaissons ni leurs localisations ni les conditions de leur action. Nous ne pouvons donc nous servir de cet exemple, pour nier actuellement les effets des hormones chez les Vertébrés, ou leur réalité et leur action résultent d'expériences indiscutables. Enfin, el surtout, si le gynandromorphisme est difficile à concilier avec des actions hormoniques s'exerçant directement, il ne me semble pas que la difficulte subsiste, en admettant que les hormones agissent, par l'intermédiaire du système nerveux, en érotizant tels ou tels centres particuliers. Sans entrer ici dans le détail de la discussion, notons qu'on peut fort bien imaginer que leur action soit limitée à certains de ces centres et ne retentisse morphologiquement que sur des portions limutées de l'organisme, produisant ainsi le gynandromorphisme.

Les expériences n'ont permis d'altérer que dans une 
certaine mesure, inégale suivant les animaux considérés, les caractères des sexes. En chàtrant le Coq, on agit sur la crète, le chant ou les instincts combaltifs; le plumage ou l'ergot ne sont pas modifiés. Mais l'intervention expérimentale n'a pu se produire qu'à une époque tardive de la vie individuelle, avant laquelle l'ébauche génitale, par l'action directe de ses hormones et par l'influence indirecte de celles-ci sur celles des autres organes, a déjà pu produire un effet morphogène considérable et définitif. Yous ne pourons, en fait, supprimer l'ébauche génitale dès son apparition réelle dans le cas du coq, c'est à un stade reculé de la vie dans l'ceuf); et même au moment où cette ébauche s'individualise, les tissus dont elle sort ont peut-être, eux aussi, déjà orienté par les mêmes processus, l'organisme rers des formes qui ne peuvent plus ètre modifiées. Si notre esprit isole des stades ou des organes, la réalité ne contient pas ces distinctions. Les actions que nous séparons par notre analyse s'exercent simultanément, depuis l'état initial de l'organisme, c'est-à-dire l'œuf; elles réagissent à chaque instant les unes sur les autres et réalisent une série d'états, dont chacun détermine, au moins dans une très large part, les suivants.

Plus nous pourrons remonter vers l'origine de cette chaîne, plus nous pourrons, selon toute vraisemblance, modifier les caractères de l'organisme définitif. En tout cas, si les hormones nous expliquent, dans une certaine mesure, le mécanisme de la manifestation actuelle des caractères sexuels secondaires. elles ne nous apprennent rien sur leur origine et sur leur différenciation. Le domaine de la physiologie est d'analyser les conditions des phénomènes actuels. La morphulogie est le résultat d'un immense passé.

Les caractères sexuels secondaires ne se distinguent 
d'ailleurs en rien des autres particulatités morphologiques chez les animaux ou les végétaux : ils ont évolué de la même façon.

L'élude expérimentale de leurs liens arec les sécrétions internes est cependant très suggestive au point de vue des conceptions actuelles sur la morphologie des organismes et des idées régnantes sur l'hérédité. Sous l'influence du weismannisme d'abord, du néo-mendélisme ensuite, la grande majorité des biologistes considère les caractères comme des unités, simples ou complexes, ayant, dès le germe, une autonomie au moins potentielle. Les données expérimentales qui précèdent nous ramènent à une conception plus saine de l'organisme : celle d'un tout indivisible, où la forme des parties et ses variations résultent de l'équilibre entre les actions réciproques des organes. 


\section{TROISIÈME PARTIE}

\section{LE PROBLĖME DU DÉTERMINISME DU SEXE GHEZ LES ANIMAUX GONOCHORIQUES}

Nous arons examiné précédemment les phénomènes de la sexualité chez les organismes à sexes séparés et vu le lien complexe qui unit la glande génitale au reste de l'organisme. Mais quels sont les facteurs qui, chez un individu donné, déterminent un sexe ou l'autre? Un animal gonochorique peut être soit mâle soit femelle, mais il n'y a pas pour lui d'état intermédiaire. Ce sont comme deux polarités séparées par une discontinuité. A quel moment et en vertu de quelles actions l'individu est-il entraîné vers l'une ou vers l'autre?

Il y a là un des problèmes les plus captivants de la biologie générale. Point n'est besoin non plus d'insister sur son intérèt extra-scientifique, quand on le considère chez les animaux supérieurs et surtout chez l'Homme. Il a été posé longtemps avant que la biologie scientifique fût constituée et, déjà au milieu du $\mathrm{xvII}^{\circ}$ siècle, le médecin Drélincourt en dénombrait 262 solutions. Ce nombre même suffit à indiquer que la bonne n'était pas trouvée. En faisant pareil dénombrement aujourd'hui, on en dresserait une liste bien plus longue et aussi décevante. 
Mais si la question n'est pas résolue et ne semble pas devoir l'ètre de longtemps, ce n'est pas à dire que nous ne sachions rien d'intéressant à ce sujet; loin de là. Sans connaitre les facteurs précis de la détermination du sexe, nous savons maintenant, au moins pour certains organismes, beaucoup sur les circonstances où il se détermine et, par là, nous pourons renoncer à des préjugés ou à des espérances chimériques. Les dernières années ont été particulièrement fécondes en recherches et en indications que nous allons résumer. 


\section{CHAPITRE XI}

\section{LES SEXES ET LA MÉTHODE STATISTIQUE}

Lois statistiques des naissances humaines. - Egalité numérique approximative des sexes chez la plupart des espèces. Exceptions à cette règle.

Le principe d'autorégulation de Düsing. - Sa portẻe réelle. - Abus de la méthode statistique : elle ne nous permet aucune induction sur le mécanisme physiologique de la détermination du sexe.

3. - Les lois statistiques et le sexe. - On a cherché parfois à étudier le déterminisme du sexe par les méthodes statistiques. Elles interviennent ici de la mème manière que dans les problèmes de l'hérédité. Elles visent à éliminer la difficulté fondamentale de la question, - la complexité même du déte:minisme qui rend les cas individuels différents les uns des autres, - en envisageant d'un seul coup des nombres très élevés d'individus. Les circonstances secondaires, particulières à chaque cas, se compensent, ne laissant subsister que les facteurs les plus généraux.

Il est beaucoup de phénomènes qui nous apparaissent avec des lois globales simples et que nous sommes amenés maintenant à concevoir comme la résultante d'une infinité de phénomènes élémentaires, dont le déterminisme est pratiquement impos- 
sible à suivre et semble obéir seulement au hasarl. La théorie cinétique des gaz en physique en est un exemple classique. Les pressions exercées par les gaz suivent des lois simples, comme la loi de Mariotte, et cependant, nous concerons ces pressions comme résultant des ćhoes désordonnés de milliards de molécules contre les parois où la pression s'exerce. Envisagés isolément, les déplacements des diverses molécules n'obéissent à aucune loi accessible pour nous, et pourtant le résultat global en est parfaitement déterminé.

Statistique des sexes dans l'espere humaine. La statistique met en éridence un fait analogue pour le déterminisme du sexe. Il nous est actuellement impossible de prévoir le sexe d'un individu donné; dans notre ignorance, ce sexe se présente à nous comme s'il était dû au hasard, et cependant si nous considérons un nombre très considérable de naissances dans l'espèce humaine, le rapport entre le nombre des garçons et celui des filles est déterminé. Le résultat de tous ces hasards individuels est une proportion constante entre les sexes, dans des conditions données, et qui varie d'une façon régulière, corrélativement à des circonstances sociales.

Ainsi, dans les pars cirilisés, tels que les nations européennes, où des statistiques précises, portant sur des millions de naissances ont été faites depuis un siècle, on constate d'une façon régulière un léger excédent de naissances masculines. Il nait 106 garçons pour 100 filles. Depuis un siècle, en France, cet excès de naissances masculines tend à s'abaisser d'unt' façon régulière. On a pu rapprocher ces variations et d'autres, de celles des conditions économiques et constater que ce qu'on est convenu d'appeler le progrès général de la richesse publique est accompagné d'une 
lécroissance des naissances masculines par rapport aux féminines.

La mortalité des enfants mâles est plus élevée que celle des filles, car la statistique montre qu'à l'âge adulte, les deux sexes sont représentés en nombres sensiblement égaux. De même la statistique des mort-nés montre d'une façon constante que la proportion des garçons y est plus forte que celle des filles et que l'écart est très supérieur à celui cité plus haut entre les sexes des nés-rirants. Dans la plupart des pays, on compte, con effet, au moins 130 mortnés masculins pour 100 mort-nés féminins.

Ces données, et d'autres qu'on a pu établir en envisageant les statistiques à des points de vue particuliers, révèlent donc des faits généraux constants, susceptibles de prévision pratiquement certaine, des lois relatires à la sexualité dans les naissances humaines.

Égalité numérique approximatice des sexes chez la plupart des animaux. - Des statistiques analogues existent, quoique sur une échelle moindre, pour les animaux domestiques et conduisent à des résultats de même ordre. Ainsi, pour les cheraux, la compilation des stud-books des haras indique que sur un total de l'ordre de 1.000 .000 de naissances, il nait 96,5 mâles pour 100 femelles, écart inverse de celui qu'offre l'homme, mais également assez constant.

Pour les animaux sauvages, on n'a naturellement pas de statistiques analogues suffisamment importantes. Le dénombrement des mâles et des femelles dans un lot considérable d'individus recueillis à un moment donné conduit en général, si les nombres sont suffisamment élevés, à des proportions sensiblement égales des deux sexes. Mais le renseignement ainsi obtenu est moins précis que les précédents, car 
l'égalité entre les sexes peut résulter d'une mortalité excessive de l'un d'entre eux au début de la vie ou du développement. La statistique a mis précisément en évidence un phénomène de cet.ordre pour l'homme, comme nous venons de le voir.

En tout cas, réduits même à leur portée légitime, ces dénombrements n'ont de valeur que s'ils portent sur des nombres très considérables. Il est vraisemblable que chez la majorité des espèces, les mâles ef les femelles sont représentés en nombres sensiblement égaux et on admet, abusivement peut-être, que les deux sexes sont produits dans un rapport également voisin de l'unité.

Si cela peut être considéré comme une règle assez générale, il n'est pas douteux qu'elle ne comporte de nombreuses exceptions. On connait de façon positive un certain nombre d'espèces, oủ mảles et femelles sont inégalement nombreux. Dans certains Ephémères par exemple, on troure on n'indique pas malheureusement sur quels nombres totaux a porté la statistique) 60 màles pour une femelle. Chez divers Papillons, tels que Papilio agesilaus, $P$. protesilaus, les femelles sont des raretés, alors que le màle est commun; et cette circonstance se traduit par une conséquence qui est un signe assez significatif : sur le marché commercial des Insectes, il y a une différence de prix énorme entre les deux sexes.

Pour certains animaux qu'on peut observer en réritables cultures, tels que les Rotiferes, les Crustacés Cladocères, on sait, d'une façon sûre, que pendant des périodes plus ou moins considérables il n'existe aucun mâle. Enfin il est des espèces longuement observée:par des spécialistes, dont le mâle n'a jamais été vu et n'existe peut-être que dans des circonstances tout a fait exceptionnelles. Mais tous ces derniers cas relevent d'un régime tout spécial au point de vue de 
la sexualité; ce sont des animaux parthénogénétiques et nous étudierons ce phénomène à part.

La rareté de l'un des șexes, mème constatée sur des nombres tris élevés dindividus, n'est toutefois pas nécessairement la preuve décisive de sa rareté véritable dans la nature. Il se peut en effet que ce sexe ait des mœurs particulières et échappe automatiquement, gràce à elles, aux procédés de capture mis en curre. Une inégalité numérique importante entre les mảles et les femelles d'une espèce peut donc être simplement un indice d'une circonstance de ce genre.

C'est ce qu'illustrera bien un exemple. Tout le monde a ru, dans l'air, exécutant des sortes de danses rapides, des essaims de petits Insectes, des Moucherons, dit le vulgaire; ce sont en général des Diptères. Or, ces essaims ne comprennent guère que des màles et divers observateurs, en particulier J. Pérez, ont montré que ces mảles cherchent des femelles pour s'accoupler. En capturant, d'un coup de filet, un nombre considérable de ces Insectes, on ne trouvera done que des màles, ou. en tout cas. une proportion infime de femelles; d'où on sera porté à conclure que celles-ci sont bien plus rares que ceux-là. Cela n'est cependant nullement certain. C'est ainsi que Keilin, étudiant, dans ces conditions, des Trichocères, genre particulier de Diptères, sur 150 individus capturés au vol n'a pas récolté une seule femelle. En réalité, celles-ci ne quittant guère leurs abris, il faut les chercher sous les pierres. En élevant un lot de larves de la mème espèce, choisies au hasard, Keilin a obtenu plus de femelles que de mâles. On voit donc combien la récolte faite dans les conditions ordinaires donne un renseignement faux sur la proportion numérique réelle des deux sexes.

Pour ces diverses raisons, les statistiques vraiment décisires sont celles qui porteraient sur une population 
nombreuse d'individus pris tous dès l'état d'œuf ou à la naissance. Or, elles sont très rares, parce qu'elles sont pratiquement très difficiles à établir. Un auteur américain, Montgomery, en a fourni une, dans ces dernières années, pour une Araignée Lathrodectes mactans. Chez un certain nombre d'espèces d'Araignées, les mâles sont beaucoup plus petits que les femelles et plus éphémères; en revanche ils sont plus nombreux. Montgomery a fait pondre Lallirodectes mactans en captivité; les Araignées déposent, comme on sait, un groupe d'œufs dans un cocon soyeux vù ils se déreloppent; on peut reconnaitre le sexe chez Lathrodectes au moment où les jeunes éclosent. Chaque cocon peut renfermer jusqu'à 350 cufs; 127 cocons examinés ont fourni 37.210 màles contre 4.539 femelles, soit un rapport global du nnmbre des màles à celui des femelles égal à 8,19 . Des cocons de la mème espèce recueillis dans la nature, ont fourni 3.866 mâles pour 223 femelles, ce qui donne un rapport 17,3. Si l'on considère la statistique de chaque cocon isolément le rapport varie de 2 à 27 . Je cite ces chiffres qui montrent que, dans cette espèce, il $\mathrm{y}$ a éridemment prédominance numérique des màles, mais que si on la mesure sur des nombres peu élevés, elle se traduit par des chiffres extrèmement rariables. Ils ne peurent areir de signification véritable que sur des populations très considérables.

\section{5. - Valeur réelle des lois statistiques sur les} sexes. - La statistique, convenablement pratiquée, nous apprend donc que, dans chaque espèce, il y a une proportion assez constante du nombre des individus màles et femelles produits et que, dans la plupart des cas étudiés, cette proportion est très voisine de l'unité. C'est évidemment une loi, car c'est l'expression d'un rapport fixe entre des phénomènes et 
on peut en déduire des prévisions. Mais il ne faut pas forcer la valeur d'une loi de ce genre. Elle signifie seulement que les différents facteurs, dont l'ensemble détermine le sexe dans l'individu, jouent, les uns par rapport aux autres, dans des conditions qui se compensent finalement.

Düsing, à qui on doit un volumineux travail sur ces questions, chez l'Homme et dans le règne animal, interprète le résultat global comme la preuve de l'existence d'un mécanisme autorégulateur de la proportion des sexes dans l'espèce. Il rattache ce principe à la théorie darwinienne de la sélection naturelle, qui était à l'apogée de la fareur, lorsqu'il fit son travail vers 1880. La proportion réalisée par le mécanisme autorégulateur serait celle qui est la plus arantageuse à la conservation de l'espèce.

C'est là une constatation synthétique a posteriori, mais non un principe fécond, d'où on puisse induire d'autres résultats, comme nous le verrons tout à l'heure. Elle a une valeur réelle comme expression globale des faits, permettant de les saisir dans leur ensemble et d'en aperceroir des éléments permanents. A ce titre, elle aura une valeur pratique; elle pourra serrir de base à des calculs de probabilité, à des applications à des problèmes d'assurance, de mortalité, etc., et à l'étude de phénomènes sociaux. Mais il ne faut pas lui demander autre chose, ni voir, dans la méthode statistique, la clé des problèmes biologiques du déterminisme du sexe.

Cette méthode statistique suffirait, en effet, à nos besoins si nous ne pouvions envisager que le phénomène global, comme dans le cas de la pression des gaz, où nous ne pourons arriver à la considération des molécules isolées; le fait global est alors le seul qui importe à notre esprit. Mais il n'en est pas de même ici.

Pour compliqué que soit chaque cas individuel, il 
nous est accessible et jusqu'à un certain point analysable. Les groupements secondaires de cas découpés dans l'ensemble le sont à plus forte raison. Or, les lois statistiques et le principe d'autorégulation de Düsing ne sont d'aucune utilité pour éludier les uns ou les autres. Ces lois n'ont pas une existence intrinsèque; elles n'existent plus pour ces faits particuliers. Et, quand on a voulu se baser sur elles pour expliquer ceux-ci, on n'est arrivé qu à des pétitions de principe, à des interprétations ahnsives ou à des contradictions.

Voyons, par exemple. comment Düsing fait interrenir le principe autorégulateur qu il a formulé, pour expliquer des manifestations particulières dela sexualité. Certaines statistiques indiquent, dans les naissances fournies par des primipares àgées, un excès de garçons. Düsing les interprete en admettant que le retard dans l'accomplissement de la reproduction a mis les femmes en question dans des conditions phrsiologiques analogues à celles des femelles d'espèces où il y aurait pénurie de mâles. Dès lors, le mécanisme autorégulateur doit intervenir pour compenser cette pénurie en déterminant la production de màles en excès. C'est ce que la statistique constaterait. Mais y a-t-il là véritablement une relation de cause à effet? En premier lieu, les statistiques de ce renre sont suspectes et rien ne dit que leur résultat soit bien constant dans le sens indiqué. En second lieu, l'assimilation physiologique faite par Düsing est des plus ragues et des plus discutables. Le sens attribué à la corrélation en question est donc loin d'ètre justifié. De mème, les grandes guerres, éliminant brusquement un grand nombre d'hommes en àge de reproduction, créent un déficit de mâles appréciable dans un pays; en vertu du même principe, il doit y avoir, après ces guerres, excès de naissances masculines. A 
l'appui, Düsing, en 1884, indiquait, sans précision d'ailleurs, le cas de l'Allemagne après les guerres napoléoniennes. Mais on n'a rien constaté d'analogue après 1870, et Bugnion a fait examiner, à cet égard, les statistiques des naissances, au Japon, avant et après la guerre de 1904-1905 avec la fussie. Le résultat est inverse. La proportion des naissances mâles est plus faible après la guerre qu'avant ${ }^{1}$.

\$3. - Curnclusions. - Le défaut de tous ces raisonnements est qu'il n'y a pas de parité entre le déterminisme physiologique du sexe qui, pour être très compliqué et encore inconnu, ne doit pas moins être précis, et les circonstances sociales invoquées : cellesci sont mal définies; les cas individuels qu'elles englobent ne sont pas réellement comparables entre eux.

Lierreur de raisonnement consiste à vouloir remonter du fait global; qui peut ètre incontestable, à la phycsiologie du déreloppement de l'ceuf.

Ainsi, tout récemment, R. Worms, rérifiant, sur la natalité française depuis un siècle, leslois statistiques des naissances masculineś et féminines, constate un parallélisme entre les variations du rapport des sexes et celles des conditions sociales envisagées dans leur ensemble. L'accroissement de la richesse publique, donnée d'ailleurs peu précise, est corrélative d'un accroissement de la proportion des naissances féminines. Lo rapprochement de ces deux données statistiques est licite; mais si l'on veut, comme l'auteur, rattacher l'un àl'autre ces deux faits par un mécanisme physiologique, roir la cause de l'accroissement des naissances femelles dans des conditions meilleures de nutrition pour les embryons et conclure qu'une nutrition défectueuse favorise la naissance des mâles, une

1.

$$
\left(\frac{O}{\varphi}=\frac{104}{108} \text { de } 1896 \text { à } 1903 ; \frac{101}{100}\right. \text { en 1907.) }
$$


nutrition meilleure celle des femelles, on dépasse la pa. tée des faits et l'on transforme une coïncidence en une relation de cause à effet: post hoc, ergo propter hoc.

C'est en commettant cette faute que beaucoup d'auteurs sont arrivés à de véritables puérilités, comme de dire qu'après des années prospères il y arait excès de naissances féminines ou que la morti-natalité plus grande des embryons mâles tenait à ce qu'ils sont concus dans des conditions moins favorables.

Les variations des conditions économiques ne peuvent rien faire préjuger quant à la nutrition de l'embryon. Quand bien même l'organisme maternel souffrirait de conditions économiques mauraises, l'embryon n'en serait pas nécessairement atteint. Tout le dommage pourrait être supporté par l'organisme maternel, sans retentir sur le fruit lui-même. En tout cas, une analyse physiologique précise autoriserait seule à décider ce point. Les corrélations statistiques ne permettent de rien inférer directement. quant aux mécanismes physiologiques.

La méthode statistique est légitime, à condition de ne lui demander que ce qu'elle peut donner. On ne saurait trop relire ce qu'a écrit Claude Bernard ${ }^{1}$ sur son application aux problèmes biologriques. Elle ne peut nous révéler la nature même des phénomènes. mais seulement leurs conséquences quand on envisage l'ensemble de leur accumulation. Nous ne pouvons nous représenter leur nature que par l'analyse des conditions qui les déterminent dans des cas isolés ou des groupements de cas strictement comparables. Il va sans dire que, dans cette analyse, la statistique intervient, à certains moments, comme moyen. C'est ainsi que le problème biologgique du déterıninisme du sexe doit être abordé.

1. Introduction à la Médecine expermentale ( $2^{\mathrm{e}}$ partie, $\mathrm{ch}$. $\mathbf{n i}$, §. pp. 226-246). 


\section{CHAPITRE XII}

\section{L'ÉPOQUE DE LA DÉTERMINATION DU SEXE}

Le sexe doit être déterminé avant d'être effectivement reconnaissable. - Trois hypothèses générales possibles sur le moment de sa dėtermination : après, avant ou à la fécondation (épigamie, progamie ou syngamie).

Hypothèse épigamique. - Expériences sur les Papillons, les Mouches, les Batraciens. - Conclusions négatives. - Les phénomenes de polyembryonie sont un argument indirect contre la détermination épigamique. - Expériences diverses sur les Végétaux.

§1. - Conditions du problème. - En se plaçant au point de rue biologique, la première question à résoudre est de chercher à quel moment de la vie individuelle se détermine le sexe, ou plutôt à partir de quel moment il est irrérocablement déterminé. Car on fera justice ainsi de toutes les solutions proposées qui supposaient implicitement une détermination plus tardive qu'elle n'est en réalité.

La difficulté de ce problème est que le sexe peut ètre déjà déterminé sans être cependant reconnaissable. Si on se borne à l'aspect extérieur, comme on doit le faire avec des individus vivants, la distinction des sexes peut n'ètre possible qu'à l'état adulte ou au moins à l'âge de la première reproduction. C'est le cas pour certains Diseaux dont le sexe est pratiquement très difficile à reconnaitre plus tôt. Mais, si l'on 
peut faire des constatations anatomiques proprement dites, la structure des organes génitaux externes, appareil copulateur du mâle ou vagin de la femelle, permet de reconnaître le sexe de fort bonne heure, même pendant la vie embryonnaire. Le moyen le plus sûr et le plus précoce, sur les cadarres, est l'étude histologique de la glande génitale elle-même. La nature de ses éléments germinaux rérèle, dès les phases reculées de la vie embryonnaire, si elle produira des spermatozoïdes ou des ovules; parfois même, la disposition des tissus accessoires de ces glandes, comme, chez les Vertébrés, celle du tissu interstitiel, permet de diagnostiquer le sexe, arant même que les éléments germinaux proprement dits n'offrent une indication décisive. C'est ainsi que, pour l'homme, le sexe femelle de l'ébauche génitale peut être reconnu sur des embryons de 11 à 13 millimètres de longueur, correspondant à la quatrième semaine de la vie fœtale. La forme de l'ébauche de l'ovaire est alors légèrement différente de celle du testicule, sans qu'on puisse encore reconnaître, sur les éléments germinaux proprement dits, s'ils doivent évoluer en spermatozoïdes ou en ovules.

Mais il va sans dire que le sexe pouvait ètre fixé antérieurement; il est même vraisemblable qu'il l'est bien avant le moment précis où nous en trourons, dans nos connaissances actuelles, un critérium visible. Avec les procédés actuels de l'histologie, nous possédons déjà ce critérium, bien plus tòt qu'avec lez seules constatations purement anatomiques dont on disposait autrefois.

En fait, il y'a, chez la plupart des animaux, une première phase, où l'ébauche de la glande génitale paraît indifférente ${ }^{1}$, et c'est secondairement qu'elle so

1. Nous avons vu, (cf. p. 15) que l'ébauche de la glande génitale, abstraction faite du sexe, peut ètre distinguée de très 
différencie vers un sexe ou rers l'autre. Cette indifférence primaire apparente peut fort bien être réelle. Rien ne force a priori à admettre que le sexe est déterminé dès l'état d'œuf. Nous rerrons cependant que de bonnes raisons semblent indiquer qu'il en est ainsi, pour plusieurs groupes d'animaux.

J'ajoute enfin qu'a priori, nous derons ètre en garde contre la tendance très naturelle à supposer une solution unique et générale à ce problème. La détermination du sexe peut se faire à des moments divers, suivant les groupes considérés.

L'état d'indifférence sexuelle primitive réelle cadrerait assez bien avec l'existence de l'hermaphrodisme. Le fait, en effet, que les deux catégories de garnètes se forment côte à côte normalement, chez de nombreux organismes, que l'hermaphrodisme réapparait, d'ailleurs à titre exceptionnel et rudimentaire, chez les types gonochoriques, rend plausible que l'ébauche primitive de la glande génitale soit réellement indifférente et qu'elle soit orientée dans un sens ou dans l'autre par une circonstance extérieure à elle, par un état de l'organisme qui influerait sur elle. Bouin et Ancel, par exemple, admettent l'idée que ce pourraient être les éléments interstitiels qui, par le chimisme de leur fonctionnement vital, détermineraient, à un certain moment, les éléments germinaux primordiaux à évoluer vers la spermatogénèse ou vers l'orogénèse.

On pourrait mème concevoir, sans trop de paradoxe, le sexe de l'embryon comme virtuellement fixé dès le début du développement, dès l'état d'œuf, si cela résultait indirectement des faits, et l'ébauche génitale elle-

bonne heure, que, chez divers types, on distingue les cellules génitales primordiales, dès la segmentation de l'œuf, et même dès le début de la segmentation (stade de 2 cellules chez Ascaris megalocephala; cas divers chez les Insectes). 
même, comme étant cependant tout d'abord réellement indifférente. Le sexe pourrait être une propriété de l'organisme, dans son ensemble, avant d'être une propriété de la glande génitale. Cette hypothèse est, ḋ la vérité, peut-être bien subtile; je la fais pour montrer combien la solution complète du problème posé, - fixer le moment véritable de la différenciation du sexe, - est ardue, pour ne pas dire impossible. N'es. pérons donc que des solutions approchées.

Tous les cas possibles peurent ètre classés chronologiquement, par rapport au moment de la formation de l'œuf, à la fécondation, qui est une étape décisive. On est done ramené à envisager les trois hypothèses suivantes qui pourront, a priori, être exactes, suivant les types envisagés:

$1^{\circ}$ La détermination du sexe est antérieure à là fécondation; le sexe est déjà fixẻ dans l'ovule.

$2^{\circ}$ Elle est contemporaine de la fécondation; elle résulte de la composition de l'œuf à la suite de la fusion des deux gamètes.

$3^{\circ}$ Elle est postérieure à la fécondation.

V. Hæcker a proposé, pour ces trois possibilités, les désignations assez claires de détermination progame, syngame ou épigame du sexe.

Quels sont les faits qui plaident en faveur de l'une ou de l'autre? Nous envisagerons d'abord l'hypothèse de l'épigamie.

§ 2. - Examen de l'hypothèse épigamique. - Le sexe se délerminerait seulement au cours du déreloppement de l'embryon. C'est l'hypothèse qui a été considérée tout d'abord comme la plus plausible. Cela est naturel. Elle répond aux apparences, puisque le sexe ne peut ètre reconnu qu'à partir d'un stade plus ou moins avancé. D'autre part, comme le déterminisme du sexe a été toujours envisagé arec l'ar- 
rière-pensée d'en découvrir le mécanisme chez l'homme, c'est la solution qui était sentimentalement désirée. Si le sexe, en effet, ne se détermine que pendant le développement, on peut espérer agir sur les conditions d'où il lépend. Enfin, il était a priori admissible qu'il pût ètre influencé par des circonsfances telles que la nutrition.

Sous l'empire de ces préoccupations, les moyens proposés pour influer sur le sexe n'ont pas manqué. A la théorie épigamique se rattachent une foule de préceptes empiriques ou basés sur des raisons physiologiques plus ou moins précises. On se rappelle encore le retentissement qu'eut, vers 1900, la théorie de Schenck. Ce médecin prétendait avoir reconnu dans le taux des substances azotées éliminées par la mère dans l'urine, pendant la grossesse, un signe certain du sexe du fœtus. En agissant sur la nutrition de la mère, pendant quelques mois avant la conception et quelques semaines après, de façon à atteindre un taux déterminé de substances azotées dans l'urine, Schenck affirmait inffuer sur le sexe. La théorie, physiologiquement contestable, n'a pas résisté à l'épreuve des faits; il n'y a pas lieu de s'y arrêter. Tout récemment, Robinson a déduit d'expériences faites sur des Cobayes et de quelques observations cliniques, que l'injection d'adrénaline à l'époque des débuts du développement farorisait la production d'individus mâles; mais, à défaut d'autres considérations, les essais sont encore beaucoup trop restreints pour permettre des conclusions sérieuses.

Des expériences assez nombreuses ont été faites sur divers animaux. Bien qu'aujourd'hui leur valeur soit en général controuvée, il n'est pas inutile de résumer les plus connues.

Leur principe est le suivant : on prendra un lot d'individus aussi nombreux que possible et bien com- 
parables, - par exemple, les œufs et embryons d'une même ponte; on en fera trois lots, dont l'un sera nourri abondamment, l'autre déficitairement; le troisième, qui servira de témoin, normalement. On maintiendra toutes les autres conditions exactement égales. A la fin de l'expérience, on constatera le pourcentage de mâles et de femelles dans chacun des lots, et on verra si la nourriture a influé.

Les difficultés expérimentales sont, en réalité, très grandes. La première est de disposer de lots suffisamment nombreux, condition indispensable. La seconde, non moins grande, est de conduire les élevages sans mortalité importante; car, s'il y a une grande mortalité au début, elle peut avoir porté en excès sur l'un des sexes et les chiffres finaux n'indiqueront pas la proportion réelle de ceux-ci dans les lots. Enfin et surtout, il est très difficile de réaliser l'équivalence des diverses conditions d'élevage autres que la nourriture.

Expériences sur les chenilles, etc. - C'est aux Papillons qu'on s'est surtout adressé, les chenilles étant, parmi les larves, les plus faciles à nourrị.

Landois (1867), Miss Mary Treat (1873), Gentry (1873) ont élevé diverses espèces de chenilles, et notamment celles de Vanessa urticæ, en faisant varier la quantité de nourriture. Ils avaient conclu qu'une alimentation faible produisait un excès de mâles. Mais ce résultat n'a pas été confirmé par des expérimentateurs postérieurs, tels que Riley, Bessels, Briggs, Andrews, Fletscher, Standfuss, Cuénot. Sans entrer dans la discussion détaillée de ces direrses expériences, il suffit d'opposer aux conclusions positives des premiers observateurs, une donnée inconnue il y a quarante ans, mais aujourd'hui bien établie et qui les rend illusoires. A l'âge où l'on prenait ces chenilles pour 
tàcher d'influer sur leur sexe, elles ont déjà des - Flandes génitales de sexe irrérocablement et nettement déterminé ${ }^{1}$. La nutrition n'a donc pas pu exercer ultérieurement d'action véritable. Les résultats enregistrés par les premiers observateurs tiennent à des circonstances qui ont passé inaperçues : sélection inconsciente, au début, de chenilles de l'un des deux sexes, mortalité excessive des femelles ou des mâles au cours de l'expérience, etc... Tout au plus résulterait-il d'expériences de Pictel², que l'alimentation fournie à une chenille peut influer sur le sexe des générations suivantes. P. Marchal a énoncé un résultat du même ordre pour un Diptère, la Cécidomyie du froment. Mais ces résultats rentreraient dans le cadre de la détermination progamique.

Cuénot, en faisant varier l'alimentation des larves de diverses Mouches très communes ${ }^{3}$, n'a obtenu aucun changement significatif dans la proportion des sexes : il réussissait seulement à modifier la taille de ces mouches.

Expériences sur les Batraciens. - Un cite souvent des expériences analogues faites sur les Batraciens (Grenouilles, Crapauds); mais aucun résultat n'est décisif en faveur d'une action effective de la nour-

1. Cela réfute aussi les conclusions tirées par C. Flammarion d'expériences sur l'influence des radiations de diverses couleurs, pendant la vie de la Chenille, sur le sexe du Papillon.

2. Pictet a expérimenté notamment sur Lymantria dispar. Un lot de Chenilles, nourri avec des feuilles de noyer (alimenıation défectueuse) donne $5 \%$ \% mâles et $46 \%$ femelles et $65 \%$ mâles et $3 \breve{a} \%$ femelles à la génération suivante. Un autre lot nourri avec de l'esparcette et du Leontodon (ce qui constitue ane nourriture favorable) donne $50 \%$ de mâles et $49 \%$ de femelles à la seconde génération.

3. Calliphora vomitoria, Lucilia cæsar, Sarcophaga carnaria, etc. 
riture sur le sexe. D'ailleurs, si ces animaux offrent des facilités pour l'obtention de pontes nombreuses en individus, ils présentent des inconvénients difficilement surmontables. On ne peut guère éviter une forte mortalité au cours des élevages; en outre, le sexe ne peut être reconnu avec certitude, au moins chez la Grenouille rousse (Rana temporaria) généralement employée, pour diverses raisons, que vers l'àge de deux ans. Un certain nombre d'individus commencent, en effet, par présenter des ovules dans les glandes génitales et deviennent cependant ensuite des mâles. Il y a là une ébauche d'hermaphrodisme, qui a été constatée d'abord par Pflüger et dont R. Hertwig a cherché récemment, avec ses élèves, à préciser la nature et les conditions. Quelles que soient celles-ci, cet hermaphrodisme est un gros obstacle et fait suspecter le sens de certaines statistiques qui ont été faites sans le connaître.

Ainsi Born, en 1881, élevant des têtards de Rana temporaria avec une nourriture ariée, dans 21 aquariums distincts, avait constaté, sur 1.272 jeunes grenouilles, $95 \%$ de femelles. C'était là un écart énorme par rapport à la normale et, comme l'élerage arait été fractionné, Born en avait conclu qu'une cause extérieure, qu'il ne pouvait préciser, avait dû ag̣ir sur le sexe au cours du développement. Il y aurait donc là un fait favorable à l'idée de la détermination épigamique. Mais on peut se demander si, dans cette statistique, le sexe véritable a bien été reconnu et si un certain nombre d'individus, considérés comme femelles, n'étaient pas des hermaphrodites rudimentaires, destinés à devenir des mâles, rossibilité que Born ignorait. S'il en était ainsi, le chiffre de $95 \%$ ne représenterait pas réellement la proportion des femelles dans l'élevage de Born.

Yung, dans direrses expériences, faites de 1883 
à 1885, avait obtenu, en nourrissant des têtards avec des substances animales, un pourcentage très élevé de femelles ( 70 à $78 \%$ et mème, dans une expérience, $92 \%$. Il en avait conclu qu'une forte nutrition augmentait le nombre des femelles; Balbiani et Henneguy étaient aussi arrivés, un peu plus tard, à des conclusions analogues en nourrissant les têtards arec des jaunes ou des blancs d'œufs cuits.

Mais, ici encore, des expériences plus récentes et plus précises, celles de Cuénot sur les Grenouilles et de Miss Helen King sur les Crapauds, les ont infirmées. La proportion des sexes n'est pas modifiée par la nourriture au cours du développement; seule la taille est influencée.

Aucune des expériences précédentes, qui ont été souvent citées comme des preuves qu'une nutrition abondante des jeunes favorise la production des femelles, n'a cette portée; et, d'une façon générale, on ne connait pas de faits positifs sûrs, en faveur de la détermination épigamique du sexe chez les animaux.

Polyembryonie et détermination du sexe. - Il est, d'autre part, un ordre de faits qui, sans permettre de choisir entre les deux autres catégories, progamie et syngamie, et tout en étant limité à un petit nombre de cas, est également en opposition avec l'épigamie. Je veux parler des phénomènes de polyembryonie. On désigne ainsi la fragmentation de l'embryon issu d'un œuf unique, en embryons secondaires, qui deviennent autant d'individus définitifs. S. F. Harmer a, le premier, fait connaître ce phénomène chez les Bryozoaires cyclostomes, où il semble avoir une certaine généralité; ces animaux étant hermaphrodites, nous n'avons pas à nous en occuper ici. P. Marchal, à son tour, l'a décourert chez certains Hyménoptères qui pondent dans l'œuf ou la larve d'autres Insectes, 
par exemple chez Encyrtus fuscicullis, parasite d'une chenille, la Teigne du fusain. In seul œuf produit une centaine d'Encyrtus. Or, les individus provenant d'un même œuf sont toujours de même sexe. Ce sexe paraît donc être une propriété de l'œuf, antérieure a l'individualisation des embryons, c'est-à-dire, comme celle-ci remonte au début du développement, contemporaine de l'état d'œuf lui-même.

Parmi les Mammifères, la polyembryonie était soupçonnée depuis quelques années chez les Tatous. Elle y a été mise hors de doute par les recherches récentes de Fernandez et de Newman et Patterson. A un stade très précoce du développement, connu en embryogénie sous le nom de vésicule blastodermique ou blastocyste, il se différencie plusieurs embryons au lieu d'un. Or, ici encore, tous les individus issus d'un même œuf sont du même sexe.

Dans l'espèce humaine, les jumeaux vrais, que l'on reconnait à la disposition particulière des enveloppes embryonnaires, dérivent très rraisemblablement d'un œuf unique; ils sont toujours du même sexe. Les faux jumeaux, au contraire, qui naissent en mème temps, mais arec des enveloppes fœtales indépendantes, et qui proviennent vraisemblablement d'œufs distincts, sont tantôt du même sexe, tantôt de sexes opposés.

Sans entrer dans plus de détails, nous dirons done que, dans les divers cas de polyembryonie, chez les animaux à sexes séparés, tous les individus issus d'un même œuf semblent bien être de même sexe; que, par suite, la détermination de ce sexe doit ètre antérieure à la division en embryons secondaires, c'est-àdire, en fait, au développement. Elle n'est donc pas une propriété épigamique.

On pourrait, il est vrai, être tenté d'objecter à ce raisonnement que, si le sexe est le mème chez tous ces individus, c'est qu'ils se développent dans des 
ronditions identiques. Mais cette identité n'existe ni plus ni moins que pour les divers embryons se développant simultanément dans l'utérus des Mammifères a portéés multiples, et chacune de celles-ci comprend cependant des mâles et des femelles.

Expériences sur les plantes dioiques (Phanérogames, Mousses). - Toute la discussion précédente est relative aux animaux. Elle n'est pas, ipso facto, applicable aux régétaux; la sexualité y a une allure tout autre. Chez certains d'entre eux, des actions extérieures appliquées pendant le déreloppement de la plante paraissent susceptibles d'influencer la sexualité, qui serait par suite modifiable épigamiquement. Mais, en réalité, il s'agit, dans les divers cas, d'organismes hermaphrodites, chez lesquels on arrive à rendre un des sexes rudimentaires, ou à le masquer complètement, et non pas à produire un sexe au lieu de l'autre.

Ainsi, chez les Fougères, l'appareil sexué (prothalle) est normalement hermaphrodite. En cultivant des prothalles de l'Osmonde royale, sur des milieux dépourrus de substances azotées, Frank a constaté qu'il ne s'y formait que des organes mâles (anthéridies) et pas d'organes femelles (archégones). Une nutrition réduite transforme donc l'hermaphrodisme normal en une sexualité màle, c'est-à-dire supprime le sexe femelle. C'est bien une altération de la sexualité, mais non une interversion des sexes. Les phénomènes observés par Blaringhem, sur le Maïs, à la suite de traumatismes qui modifient les conditions de nutrition de la plante, sont du mème ordre.

Molliard a obtenu des résultats en quelque sorte inverses des précédents, sur le Chanvre et la Mercuriale, qui sont normalement diö̈ques. En semant du Chanrre en serre et en le cultivant à éclairement 
réduit, il a eu un excès de pieds femelles; c'est-à-dire que, sur de nombreux pieds, on trouvait, à des degrés variables, des étamines transformées en carpelles. En réalité, la modification de lả sexualité consiste ici en une réapparition de l'hermaphrodisme et non en une inversion des sexes ${ }^{1}$.

J'ajouterai aux cas précédents l'intéressante expérience faite par Bordage sur le Papayer (Carica papaya) à la Réunion. Le papayer est dioïque et sa sexualité est très visible. Les fleurs màles sont longuement pétiolées; les fleurs femelles sont sessiles. Un Papayer måle, ayant été décapité par un cyclone, arait produit latéralement des branches femelles. Bordage s'attacha et réussit à reproduire expérimentalement la mème transformation. Il a montré qu'elle exigeait des conditions particulières. Il faut choisir un pied vigoureux, qui fleurit dès sa première année, et le décapiter arant sa première floraison ${ }^{2}$. Ce cas se rapproche davantage de la sexualité des animaux sonochoriques et constitue un exemple où on modifie le sexe de façon épigamique.

Mais, à côté des faits précédents, les régétaux véri-

1. Strasburger, qui a répété les expériences de Moiliard, est arrivé à des conclusions contraires. Suivant lui, le sexe des Angiospermes dioïques n'est pas influencé par les actions extérieures intervenant pendant la croissance de la plante; il est déterminé dès l'œuf. Cette discordance n'est pas nécessairement une contradiction véritable, mais peut être due, comme Molliard l'a fait remarquer, à ce que les conditions expérimentales n'étaient pas les mẻmes. Il faut observer que dans ces expériences sur les plantes supérieures, on fait agir les conditions spéciales de l'expérience, seulement à partir de la trermination de la graine. Or, celle-ci est déjà une plante qui a subi, depuis l'état d'œuf, un développement long et complexe. On ne part pas de l'œuf.

2. D'après mon ami le $\mathrm{D}^{\mathrm{r}}$ Marchoux, ce fait serait connu des indigènes du Dahomey, qui pratiqueraient de manière courante l'expérience de Bordage 
tablement dioïques en présentent d'autres qui, comme chez les animaux, plaident contre l'épigamie. Tels sont les résultats des recherches d'Em. et Elie Marchal sur des Mousses dioïques ${ }^{1}$. La spore des Mousses produit en germant un filament ramifié, de structure très simple, appelé protonema. De ce filament on peut, par bourgeonnement, fractionnement, etc., obtenir un grand nombre de pieds de Mousses. Or, en cultirant dans des conditions les plus rariées de température (de $10^{\circ}$ i $27^{\circ}$, d'humidité, de nutrition, de lumière (intensité forte ou faible, radiations bleues ou rouge orangé. les protonèmes secondaires issus d'une même spore. Em. et El. Marchal ont toujours obtenu rigoureusement des plantes du même sexe. C'est, à partir de la spore, un fait parallèle à celui qu'offre la polyombryonie, à partir de l'œuf, chez les animaux. Le sere apparaît donc ici comme une propriété de la spore. non modifiable par les circonstances extérieures, qui agissent sur la plante en voie de déreloppement?.

1. Barbula uzguiswlaia, Bryum argenteum, Ceratodon puryureum.

2. On peut encure citer, comme plaidant pour la détermination du sexe antérieurement au développement (et par conséquent contre l'hypôthese de l'épigamie), un autre cas signalé par Ch. Douin chez une Hépatique dioïque, Sphærocarous californicus. Les quatre spores issus d'une même cellule mère germent sans se dissocier; on a donc des groupes de quatre petites plantes juxtaposées. Or, on constate à peu près invariablement ( $\pi$ fois sur $\$ 1$ groupes étudiés; que, des quatre plantes, deux sınt mâles et denx femelles. Strasburger en conclut que la détermination des sexés se fait symétriquement, lors de la formation des quatre spores et qu'elle est, dès lors, définitive. 


\section{CHAPITRE XIII}

\section{L'ÉPOQUE DE LA DÉTERMINATION DU SEXE (suite)}

II. - Hypothèse progamique. - Théories de Beard et de Lenhossek. - Actions exercées sur l'ovule en voie de croissance expériences de Russo. - Les espèces à œufs dimorphes: cas de Dinophilus.

III. - Hypothèse strngamique. - Cas des Rotiferes (Hydatina senta) et des Abeilles. - Thẹcrie de Dzierzon : discussions récentes.

La los de Thury; théorie de la transmission croisée du sexe. - Espériences de R. Hertwig sur les Grenouilles et de Miss H. King sur les Crapauds.

1. - Examen de l'hypothèse progamique. - Les faits actuellement connus sont, d'après ce que nous renons de voir, défavorables à l'hypothèse de la détermination épigamique du sexe. Celle de la progamie n'est pas très vraisemblable a priori, au moins comme règle générale, dans les œufs qui se développent après fécondation. Si la détermination du sexe ne se fait pas au cours du déreloppement, mais est définitive dès le début, dès l'état d'œuf, comme celui-ci résulte de la fusion du spermatozoïde et de "'ovule, on concerrait mal que le sexe dépendit seuiement de l'un seulement de ces éléments et fút fixé avant leur fusion. Quand on a formulé l'hypothè de la progamie, un a toujours, explicitement ou implicitement, considéré l'ovule comme l'élément essentiel, parce que sa masse est énorme par rapport à 
celle du spermatozoïde. Le sexe de l'embryon serait fixé dans l'ovule, avant la fécondation. Mais la masse ne saurait ètre une raison suffisante, a priori, et les faits quel'on a interprétés, comme détermination progamique du sexe, doivent ètre examinés avec précision. Quelques mots d'abord de conceptions purement thẻoriques.

Théorie de J. Beard. - En 1902, J. Beard a émis l'hypothèse qu'il y aurait deux catégories d'ovules dans chaque espèce; les uns à sexualité femelle, les autres à sexualité mâle. De même, il existerait deux catégories de spermatozoïdes; mais l'une de celles-ci ne serait pas fonctionnelle et ne jouerait aucun rôle. Ce serait toujours la sexualité de l'ovule, préalable à la fécondation, qui deviendrait celle de l'embryon. Ce sont là autant de suppositions gratuites que rien ne justifie. Beard a allégué, il est rrai. à l'appui de ses idées, le dimorphisme des spermatozoïdes qu'offrent beaucoup de Mollusques Prosobranches, tels que la Paludine ${ }^{1}$. Des deux types d'éléments spermatiques, seuls les plus petits (spermatozoïdes filiformes ou eupyrènes) semblent bien être effectivement fécondateurs; on n'a tout au moins pas pu constater la fécondation par les autres (spermatozoïdes vermiformes ou oligopyrènes). Quelques exemples analogues de dimorphisme spermatique sont actuellement connus en dehors des Mollusques. Mais cela reste néanmoins une disposition exceptionnelle que rien n'autorise à généraliser. Dans les cas particuliers mèmes où elle existe, on n'a aucun indice qu'elle ait la signification que lui attribue Beard. Celle-ci reste donc une supposition gratuite.

Théorie de Lenhossek. - Les mêmes objections

1. V. p. 10 et fig. 4 . 
portent sur l'hypothèse analogue, formulée d'ailleurs presque en même temps, par Lenhossek. Il admet aussi que tous les animaux ont deux catégories d'ovules, les uns màles, les autres femelles. Cela existe effectivement chez quelques rares types tels que Dinophilus apatris (dont nous parlerons plus loin), mais il n'y a aucune raison de généraliser. D'autre part, il affirme arbitrairement que le spermatozoïde n'influe pas sur la sexualité.

Actions exercées sur l'ovule en voie de croissance. Ces hypothèses ne sont donc pas justifiées. Mais à la détermination progamique du sexe s'en rattachent d'autres moins absolues et qui sont plus plausibles. D'une façon générale, elles expriment l'idée qu'en agissant sur la nutrition de la mère, on peut influer sur les orules en roie de croissance, en modifier la composition dans un sens déterminé, alors qu'ils sont encore dans l'ovaire, et, par là, exercer une action sur l'œuf proprement dit. On pourrait mème ainsi agir sur les propriétés des ovules, d'où sortira la génération suivante.

C'est ce que plusieurs observateurs ont conclu d'expériences sur les Papillons. Pictet, par exemple, nourrissant, comme nous l'arons dit, des chenilles de Lymantria dispar avec des feuilles de noyer, qui constituent une alimentation défectueuse, a obtenu à la génération suivante un excès de mâles ${ }^{1}$. C. Flammarion, élevant successivement trois générations de chenilles de Ver à soie sous des lumières colorées, a eu un excès de mâles $(60-70 \%)$ dans les lots exposés à des couleurs foncées. Le sexe des chenilles ellesmèmes ne peut pas être influencé, d'après ce que nous avons dit plus haut, mais il est possible que leurs

1. V. p. 150 , note 2 . 
oraires aient subi une action, qui se traduirait, à la grénération suirante, par une modification dans le pourcentace des sexes. Cela reste d'ailleurs très hypothétique.

Dans ces dernières années, un auteur italien, Russo, a effectué, sur le Lapin, une série de recherches se rattachant à des idẻes analogues. Il y aurait, d'après lui, dans l'ovaire de cet animal, deux catégories d'ovules matériellement distinguables. Les uns (anaboliques), riches en lécithine; les autres (cataloliques), où la lécithine aurait été en partie utilisée et aurait laissé un résidu formé de cristaux d'acides gras. Les premiers sont, d'après Russo, des ovules à sexualité femelle, les seconds des ovules à sexualité màle. En injectant de la lécithine à des lapines, il serait possible d'agir sur la constitution des ovules, d'augmenter leur richesse en cette substance et, par suite, de faire rarier, dans l'ovaire, à un moment donné, la proportion des orules d'un sexe déterminé. Un élevage de lapins, dans les conditions normales, lui a fourni, sur 65 jeunes, 36 mâles et 29 femelles. Un élevage parallèle. dans lequel il faisait aux mères des injections de lécithine, a produit 26 mâles et 40 femelles. La différence avec le premier est dans le sens farorable à l'hypothèse; mais les nombres sont trop faibles pour aroir une portée sérieuse.

En vertu de la même idée théorique, aussitôt après la mise a bas, l'organisme maternel doit avoir consommé beaucoup de ses réserves. Dans les ovaires, de nombreux ovules seraient à l'état catabolique. En faisant féconder des femelles à ce moment, on doil donc avoir, d'après Russo, un excès de mâles dans les portées. Ces idées sont intéressantes. On admettra volontiers la possibilité d'agir, par la nutrition sous des diverses formes, sur l'état des tissus, et en particulier des ovules. Mais la vérificatinn expérimentale 
précise du mécanisme indiqué par Russo est très difficile; les données apportées juqu'à présent ne peuvent prétendre en fournir une démonstration décisive.

Espèces à œufs dimorphes. - Les seuls faits précis en faveur de la progamie sont les cas où il y a deux catégories d'œufs nettement distinctes et dont on peut affirmer que chacune produit un sexe déterminé.

Mad. Brocadello a prétendu quill en est ainsi chez le Ver à soie et que du poids de l'œuf on pourait déduire le sexe. Cuénot, qui a entrepris une vérification précise de cette assertion, en conteste le bienfondé.

D'après Reuter, chez un Acarien (Pediculopsis graminum), dont les deux sexes ont des tailles nettement différentes (mâle, $130 \mu$; femelle, $200-250 \mu$ ), les œufs seraient aussi dimorphes $(100-150 \mu$ pourles femelles, $85-110 \mu$ pour les mâles). L'écart de ces deux dimensions n'est pas encore très considérable.

Le cas classique du dimorphisme des cufs, correspondant à un dimorphisme sexuel très accentué à l'état adulte, est celui qu'offre une série d'espèces de Dinophilus. Ces animaux sont de petits organismes marins que leur organisation, d'ailleurs très simple, conduit à regarder généralement comme des Vers primitifs (Archiannélides). On peut les partager en deux catégories : l'une formée d'espèces hermaphrodites et, par suite monotypes, dont il ne sera pas question icı; l'autre, où les mâles sont nains par rapport aux femelles et d'une structure anatomique rudimentaire. Korschelt a signalé, le premier, ce dimorphisme sexuel remarquable, chez Dinophilus apatris (fig. 27) et montré que la femelle pond, dans un cocon, un groupe d'œufs, où on distingue immédiatement deux catégories différant par la taille. Les petits aufs 
donnent des mâles, les gros des femelles. Ici donc, le sexe est súrement déterminé au moment où l'œuf est pondu, par la constitution mèmp de l'ovule et les processus de l'orogénèse. Elle est done progamique.
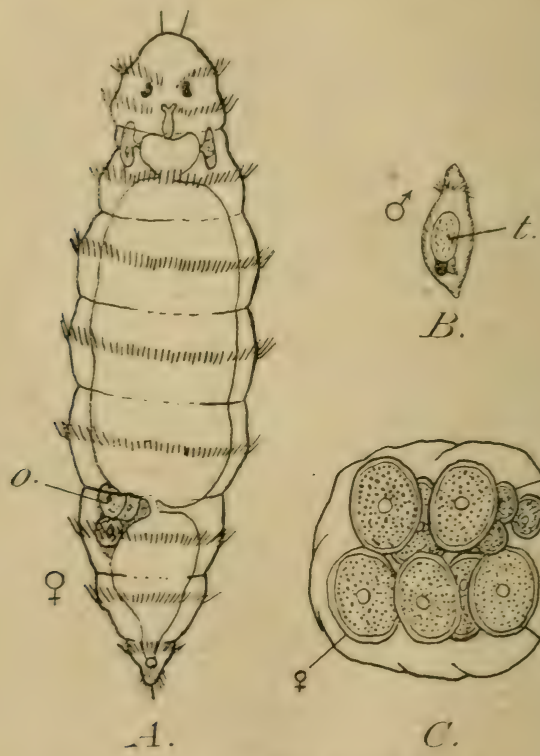

F1G. 27. - Dinophilus gyrociliatus :

A. femelle; $B$, mále (d'aprés Shearer); $C$, ponte de Dinophilus apatris d'après horschelt) montrant les deux catégories d'euts $0^{*}$ et 우; 0 , ovaire; $t$, testicule.

C'était là, tout au moins, une interprétation classique, jusqu'à ces derniers mois; mais un auteu.I anglais, Shearer, l'a remise en question. Il a montré que les femelles de ces Dinophilus sont fécondées de très bonne heure (dans le cocon même oủ elles sont en train d'achever leur développement), par leurs 
frères, dont l'existence est tout à fait éphémère; eux, en effet, meurent le plus souvent arant mème de sortir du cocon où ils sont nés. Au moment où les femelles sont fécondées, elles n'ont pas encore d'ovaire; toute l'ovogénèse s'accomplit postérieurement et les spermatozoïdes déposés par le mâle fécondent, non pas les ovules définitifs, mais des cellules initiales de lignées, d'où résultent finalement les ovules. D'après Shearer, un ovule donné éroluerait, dans l'ovaire, en un ovule mâle ou en orule femelle, suivant que, dans cette série de processus, il a ou non incorporé un spermatozoïde. Ce qui revient à dire que les ovules fécondés sont femelles et les parthénogénétiques, mâles. Le sexe n'est donc pas, s'il en est ainsi, antérieur à la fécondation, mais déterminé par celle-ci, ou par son absence. Malgré les apparences, le cas de Dinophilus apatris (et des espèces roisines), rentrerait donc dans la syngamie et non dans la progamie; il serait analogue à ce que nous verrons chez les Rotifères et les Abeilles.

Il est vrai que les conclusions de Shearer sont contestées en ce qui concerne le rôle du spermatozoïde dans la détermination du sexe, par des recherches expérimentales publiées, plus récemment encore, en juin 1912, par de Beauchamp. Cet auteur a pu obtenir, en s'entourant de toutes les précautions nécessaires, la ponte chez des femelles vierges de Dinophilus; et ces pontes, de structure et d'aspect particuliers à divers égards, comportaient les deux catégories d'œufs : œufs mâles et œufs femelles. La différenciation des sexes s'accomplirait donc bien, indépendamment de toute action du spermatozoïde et, s'il en est ainsi, son caractère progamique ne saurait ètre nié, il me semble.

Il est enfin un groupe de faits considérable, où le sexe femelle est déterminé dans l'orule longtemps 
avant sa maturité et qu'on serait par suite tenté de rattacher à la progamie. C'est celui des œufs norma. iement parthénogéniques. Chez beaucoup d'animaux, tels que les Pucerons, les Rotifères, etc., se succède une série de générations ne comportant que des femelles; le sexe des ovules est déterminé, pour ainsi dire, $a b$ initio. Mais c'est là un cas tout spécial, où la notion même de sexualité est modifiée et que nous étudierons à part 1 .

2. - Examen de l'hypothèse syngamique. - Nous arrivons maintenant à la troisième des possibilités énumérées et elle apparaît, dès à présent, comme la plus plausible. Le sexe serait déterminé virtuellement lors de la fécondation et résulterait de la constitution matérielle de l'œuf. La fusion de l'ovule et du spermatozoïde est, de toute évidence, une circonstance capitale dans la réalisation de cette constitution.

Nous avons vu, d'ailleurs, que les faits allégués en faveur de la détermination du sexe au cours du développement de l'embryon manquaient de base solide et que la progamie ne pouvait guère non plus, dans le cas général, se réclamer de faits authentiques, au moins sous sa forme absolue; cependant, elle est très admissible sous une forme atténuée : en agissant sur l'ovule en voie de formation, on modifie évidemment son état au moment de sa rencontre arec le spermatozoïde et, si le sexe dépend du conflit de ces deux éléments, le résultat de ce conflit peut en être modifié

1. Je détache toutefois ici de ce groupe de faits que, chez beaucoup de Pucerons, en particulier chez le Phylloxéra, à la génération sexuée, les mâles et les femelles proviennent d'œufs distincts par la forme. Il y a bien dimorphisme analogue à celui des œufs de Dinopliilus et remontant à l'ovogénèse; mais comme ces œufs ne sont jamais fécondés, on ne peut, à leur propos, parler de progamie, ni de syngamie. (V. chap. xvir, p. 223.) 
par là même. Sous cettc forme, les deux hypothèses de la progamie et de la syngamie se rejoignent.

Dans l'état actuel de nos connaissances, la théorie de la détermination syngamique du sexe est celle en faveur de laquelle plaident les faits positifs les mieux établis et les plus divers, et qui, par suite, semble correspondre au cas le plus général.

Cas des Rotifères (Hydatine). - La fécondation en elle-même parait exercer, dans certains types, une influence décisive sur le sexe. Il en est ainsi, par exemple, chez les Rotifères, tout au moins chez une espèce, l'Hydatine (Hydatina senta), qui a été l'objet d'expériences nombreuses.

Son mode de reproduction habituel, comme chez tous les Rotifères, est la parthénogénèse. Si l'on en fait des cultures, on voit apparaitre dans celles-ci, à un certain moment, des máles jusque-là absents. Les femelles fécondées pondent des œufs particuliers, à coque épaisse, dont le développement est très ralenti et qu'on appelle généralement, d'une façon assez impropre, œufs d'hiver, par opposition aux œufs parthénogénétiques, dits ceufs d'été1.

Or, Maupas a montré, le premier (et les recherches ultérieures semblent l'avoir définitivement confirmé), que les femelles pondeuses d'œufs parthénogénétiques sont incapables d'être fécondées. Nous dirons qu'elles sont virginipares. Ce sont des femelles spéciales qui pondent, les unes de petits œufs devenant

1. Ces deux catégories d'œufs ne sont pas nécessairement en rapport avec les saisons, pas plus chez les Rotifères que chez d'autres animaux où l'on constate des faits de meme ordre (Turbellariés, Cladocères, etc.). Les zoologistes allemands ont remplacé, avec raison, les deux termes ci-dessus par ceux de Dauereier et de Subitaneier, qui expriment que leur développement est ralenti ou immédiat. 
les mâles (pondeuses de mâles, les autres des œufs fécondables, les œufs d'hiver (pondeuses d'œufs d'hiver). Il y aurait donc, semble-t-il, trois catégories indépendantes de femelles. Iais, en réalité, les deux dernières n'en font qu'une; elles donnent des œufs d'hiver ou des mâles, suivant qu'elles ont été fécondées ou non; leur particularité véritable est d'être fécondables et de produire une génération où les sexes s'accouplent. Il vaut donc mieux les désigner sous le nom de sexupares, par opposition aux virginipares.

Elles doivent être fécondées de très bonne heure, avant que leurs œufs ne soient développés, d'une façon analogue à ce que j'indiquais plus haut pour les Dinophilus. Plus tard, la fécondation ne parait pas possible. Un auteur américain, Shull, dit aroir réussi à les féconder par un nombre insuffisant de spermatozoïdes ét, dans ces conditions, la mème femelle produirait successivement des œufs d'hiver, puis des mâles, quand la provision de spermatozoïdes serait épuisée. Cela confirmerait, d'une façon décisive, l'interprétation que je viens de résumer d'après Maupas.

La fécondation serait donc, chez l'Hydatine, - et probablement chez les autres Rotifères, - la circonstance déterminante du sexe, dans la génération où les deux sexes coexistent : fécondé, en effet, l'œuf donne une femelle; parthénogénétique, il produit un mâle. Il y a un dimorphisme marqué des œufs produisant les deux sexes, mais il est une conséquence de la fécondation et ne saurait, ici non plus, être regardé comme un fait de détermination progamique 1 .

1. Cela laisse entière la question de savoir, dans quelles conditions une femelle virginipare produit des filles virginipares comme elle, ou au contraire des sexupares. Nous verrons ce qu'on en sait à propos de la parthénogénèse. 
Cas des Abeilles. - Théorie de Dzierzon. - Le déterminisme des sexes chez l'Hydatine, tel que le révèlent ces recherches récentes, est tout à fait analogue à celui de l'Abeille et d'un certain nombre d'Hyménoptères sociaux (Guêpes, Fourmis, etc.). d'après la thénrie de Dzierzon. En raison des discussions auxquelles celle-ci a donné lieu, encore dernièrement, je l'examinerai arec quelque détail.

Une ruche d'Abeilles renferme, comme on sait, une population énorme, composée de trois catégories d'individus morphologiquement différents : la reine ou femelle féconde, unique, les mâles ou faux bourdons et les ouvrières ou femelles stériles. La reine est fécondée une sev' fois au cours de son existence, hors de la ruche, au cours de ce qu'on a appelé le rol nuptial. Après ce rol, elle rentre à la ruche, n'en sort plus et, nourrie par les ourrières, elle pond des

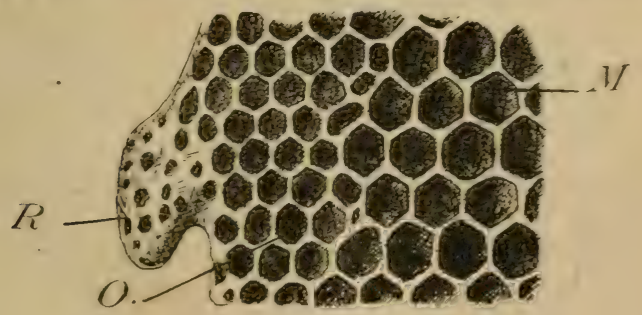

Fic. 28. - Fragment de gáteau d'Abeille :

¿q, cellule royale; $O$, cellules douvrières; $M$, cellules de mäles (sius grandes). (D'après J. Pérez.)

œufs sans cesse pendant plusieurs années. Chacun de ses œufs est déposé par elle dans une cellule des gâteaux de cire, approvisionnée de miel par les ourrières, et s'y développe en une larve qui se nourrit du miel. Les alvéoles sont, eux-mèmes, de trois formes. Sur le borci des gâteaux, de place en place, 
s'en trouve une irrégulière et plus grande : ce sont des cellules royales, et c'est d'elles que sortent les reines. Les cellules ordinaires, en prismes hexagonaux réguliers, sont de deux tailles différentes: dans les plus petites se développent des ouvrières, dans les plus grandes, des mâles (fig. 28).

Tout se passe donc comme si la reine pondait à rolonté un sexe ou l'autre, suirant la forme de l'alréole sur lequel elle se troure. Un apiculteur saxon, l'abbé Dzierzon, a expliqué cet ensemble de faits, en 1846, par l'hypothèse que la reine féconderait les œufs qu'elle dépose dans les cellules de reines ou d'ouvrières, tandis qu'elle ne féconderait pas ceux qu'elle dépose dans les cellules de faux bourdons. D'après la forme et les dimensions des alvéoles sur lesquels elle se pose pour pondre, il se produirait ou non un réflexe, d'où dépendrait l'accès des spermatozoïdes aux ovules franchissant les voies génitales ${ }^{1}$. S'il en est ainsi, le sexe, chez I'Abeille, est nécessairement lié à la fécondation : fécondé, l'œuf devient une femelle; non fécondé, il devient un mâle. Ce serait donc le même déterminisme que chez l'Hydatine.

Dzierzon s'est fondé, pour légitimer sa théorie, sur les raisons principales suivantes :

Il y a des ruches, dites bourdonneuses, dans lesquelles il ne naît que des mâles; elles sont vouées, par suite, à disparaître. Or, l'observation montre que,

1. Ceci n'a pu etre observé en fait; le mécanisme imaginé par Dzierzon ne correspond d'ailleurs pas bien aux dispositions anatomiques réelles, comme l'a montré récemment Bresslau. Il faut, en outre, imaginer qu'après la ponte de chaque œuf fécondé, il ne reste dans l'oviducte aucun spermatozoide, sinon les œufs suivants seraient fécondés, indépendamment de tout réflexe de la reine. Cela est loin d'etre évident et devrait être établi expérimentalement. On voit qu'il reste encore beaucoup à élucider sur ce point. 
dans ces cas, la reine, par suite d'une mauraise conformation, ou pour toute autre raison, n'a pu être fécondée. Pas de spermatozoïdes dans le réceptacle séminal de la reine, pas de progéniture femelle.

D'autre part, des ruches où la reine est très vieille deviennent plus ou moins complètement bourdonneuses; ce que Dzierzon explique, d'une façon analogue, par le fonctionnement défectueux du réflexe fécondateur chez les reines âgées, ou par l'épuisement de sa provision de spermatozoïdes.

En troisième lieu, dans certaines conditions, par exemple dans des ruches sans reine, les ouvrières deviennent fécondes ${ }^{1}$, mais elles ne sont pas fécondées et n'ont pas, d'ailleurs, de réceptacle séminal, où pourraient être accumulés des spermatozoïdes. Or, leur progéniture est invariablement mâle. Ici encore, pas de spermatozoïdes, pas de femelles.

Enfin, si l'on croise deux races d'Abeilles reconnaissables extérieurement à la couleur de l'abdomen, telles que les allemandes et les italiennes, la postérité mâle est du type maternel pur, alors que les reines et les ouvrières sont mixtes.

La théorie de Dzierzon souleva, en son temps, de vires discussions, mais fut généralement adoptée à la suite de travaux de vérification faits par des apiculteurs et des zoologistes, notamment par Leuckart, Siebold et Berlepsch. Siebold, en particulier, chercha la preuve directe : la présence ou l'absence du spermatozoïde à l'intérieur des œufs pondus dans les diverses catégories de cellules. Sur 52 œufs pris dans des cellules d'ouvrières, il trouva le spermatozoïde 30 fois, alors qu'il ne le rencontra dans aucun des 27 cufs prélevés dans des cellules de faux bourdons.

2. Ceci se produit plus aisẻment encore chez les Guepes. Cf. p. 94 . 
I ne faut pas oublier, qu'à ce moment, la recherche du spermatozoïde dans un œuf était très difficile, étant donné les moyens d'observation dont on disposait.

Une opposition motivée à cette théorie, malgré son succès, fut faite par J. Pérez, en 1878. Il faisait justement observer qu'on n'a pas réussi à constater, en fait, le méeanisme du réflexe fécondateur. D'autre part, un apiculteur bordelais, Drory, fournissant à une reine d'Abeilles, en automne (époque où la ponte des mâles a cessé), uniquement des cellules de mâles. n'arait obtenu que des ouvrières; donc le sexe n'est pas invariablement lié à la forme des cellules.

Enfin, Pérez fit, avec autant de précision que possible, une expérience de croisement, entre des reines italiennes et des mâles français. L'une des reines ainsi fécondées donna des mâles dès la première année, ce qui est exceptionnel. Or, sur 300 d'entre eux étudiés minutieusement, 151 étaient du type italien pur, $83 \mathrm{du}$ type français, 66 montraient une variation continue d'un type à l'autre. Il serait donc inexact de dire que, dans les croisements de ce genre, la progéniture mâle est exclusirement du type maternel.

A cette expérience de Pérez, on a objecté que la reine italienne ne devait pas être de race pure et que les mâles français qu'elle a produits constituaient un retour atavique: objection qu'on ferait plus encore aujourd'hui, en disant que ce sont les récessifs d'une disjonction mendélienne. 11 paraît d'ailleurs, qu'en 'talie même, les reines du type italien donnent parfois une progéniture màle variable et le point essentiel est d'interpréter cette variabilité. La pureté de race de la reine employée ne peut naturellement par être prouvée de façon absolue ${ }^{1}$.

: Cuénot a refait récemment (1907) un croisement analogue entre une reine noire et un màle jaune de race italienne (gol- 
En somme, Pérez ne nie pas que la parthénogénèse existe ni qu'elle ne produise des mâles; mais il conteste que tous les mâles proviennent récessairement d'œufs parthénogénétiques. Au point de vue qui nous occupe ici, le sexe ne serait donc pas lié nécessairement à la fécondation.

Cette discussion a été ranimée il y a une quinzaine d'années par un apiculteur de Darsmtadt, Dickel, praticien habile, mais ignorant des données scientifiques récentes sur la fécondation. Il a nié, beaucoup plus complètement que Pérez, la loi de Dzierzon. D'après lui, la reine ne pondrait que des œufs fécondés et le sexe serait déterminé par les qualités de la salive qui est fournie aux larves par les ourrières. On a cherché à répondre à Dickel par des constatations de faits et Petrunkevitch a entrepris, sur des œufs fournis par Dickel lui-même, de rérifier, à l'aide de la technique histologique actuelle, la présence ou l'absence du spermatozoïde dans les œufs pondus dans chaque catégorie de cellules. Les résultats des recherches de Petrunkevitch sont formellement farorables à la théorie de Dzierzon, comme le montre le tableau suivant:

\begin{tabular}{|c|c|c|}
\hline CFufs pondus dans des cellules: & Nombre: & $\begin{array}{l}\text { Spermatozoide } \\
\text { trouvé duns : }\end{array}$ \\
\hline de mâles ... $\begin{array}{c}\text { Stade. } \\
1^{\text {er }} \text { globule polaire. } \\
2^{\circ} \text { globule polaire. }\end{array}$ & $\begin{array}{l}103 \\
272\end{array}$ & $\begin{array}{l}0 \\
1\end{array}$ \\
\hline d'ouvrières. . . $\begin{array}{c}\text { Stade. } \\
\mathbf{1}^{\mathrm{er}} \text { globule polaire. } \\
2^{\circ} \text { globule polaire. }\end{array}$ & $\begin{array}{l}49 \\
62\end{array}$ & $\begin{array}{l}28 \\
62\end{array}$ \\
\hline
\end{tabular}

den-bee) sélectionnée en Amérique. Il a sbtenu des ouvrières hybrides et de nombreux mâles noirs. Cependant, quelques-uns (14 sur 300 ) montraient quelques caracteres de golden-bee. Ce résultat est, somme toute, favorable à la théorie de Dzierzon. mais les exceptions ne peuvent cependant pas être négligées; 
Cependant il y aurait encore à faire quelques réserves sur la certitude arec laquelle a été fait le diagnostic du spermatozoïde, et de nouvelles recherches ne seraient pas inutiles.

L'ensemble des faits acquis permet de conclure à la réalité de la parthénogénèse chez l'Abeille et il parait certain aussi que la parthénogénèse produit toujours des mâles; les œufs fécondés donnent des femelles, mais il n'est pas absolument prouvé qu'ils ne donnent jamais de mâles. La preuve sera d'ailleurs très difficile à administrer ${ }^{1}$.

La loi de Dzierzon s'applique, avec un degré de certitude analogue, à d'autres Hyménoptères sociaux : aux Guêpes, chez lesquelles P. Marchal a constaté d'une façon précise que les ouvrières fécondes produisent exclusivement des máles; aux Bourdons, aux Polistes (d'après Siebold), aux Osmies (d'après Fabre), aux Fourmis. Il résulterait cependant d'observations de Wheeler et de Reichenbach que, chez ces dernières, les ouvrières peuvent pondre des femelles.

Parthénogénèse arrhénotoque et thélytoque. - En admettant, an moins provisoirement, la loi de Dzierzon, la détermination da sexe, chez les espèces où elle s'applique, serait corrélative de la fécondation. Le parthénogénèse entraînerait le sexe mâle; elle serait comme on dit arrhénotoque .

Le cas inverse existerait, chez d'autres Insectes, où la parthénogénèse produirait des femelles et peut-ètre dite, par suite, thélytoque ${ }^{2 \cdot 3}$. C'est ce qui arrive chez

1. La position des œufs dans les diverses cellules n'est pas une donnée absolument sùre. On ne sait pas observer ce qui se passe dans la ruche, et on ne peut affirmer formellement que les ouvrières ne déplacent jamais les œufs pondus par la reine.

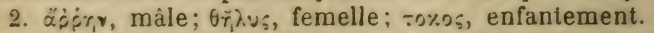

3. Dans les espèces où la parthénogénèse devient un mode normal de reproduction, au moins pendant un nombre élevé de générations, elle est d'ailleurs naturellement thólytoque. 
les Phasmides, qui appartiennent à l'ordre des Orthoptères. Dans certaines espèces de ce groupe, les mâles sont communs (ces espèces sont dites polyarrhéniques); dans d'autres ils sont très rares (espèces oligarrhéniques) et la reproduction, chez ces dernières, est généralement parthénogénétique. Dansles premières, on provoque aisément la parthénogénèse, comme l'ont constaté de Sinéty et Pantel, en séquestrant les femelles; elle y est facultative. Or, les femelles ainsi séquestrées et parthénogénétiques ne pondent que des femelles. Toutefois ce n'est pas là un fait absolu. Après huit ans d'expériences concordantes, sur un de ces Phasmides, le Dixippus morosus, Pantel a obtenu en 1907, deux mâles par voie parthénogénétique.

Loi de Thury. - Les exemples précédents doivent leur netteté à l'existence de la parthénogénèse, mais sont, par là même, d'une nature spéciale. Dans le cas ordinaire des espèces où la fécondation est obligatoire, la détermination syngamique du sexe ne peut être décelée que par des voies indirectes et la preuve en est naturellement infiniment plus difficile à donner. Il est assez logique de concevoir que la destinée de l'œuf, et en particulier le sexe de l'embryon, soient la résultante des propriétés intrinsèques des deux gamètes qui en sont le point de départ; mais, comme dans l'hypothèse de la progamie, on a été tenté d'attribuer à l'ovule un rôle prépondérant. Cet'e tendance se retrouve dans une théorie déjà ancienne, qui a eu un succès très vif tout d'abord et qui, après avoir été plus ou moins abandonnée, a trouvé récemment un regain d'actualité : je veux parler de la loi de Thury.

Thury, professeur de botanique à Genève, vers le milieu du siècle dernier, avait été amené, par des 
considérations très indirectes relatives aux plantes dioïques et qui n'ont plus aujourd'hui un sens très précis, à conceroir que, chez les animaux, l'ovule passe par des phases successives de maturité, où il a, d'abord la tendance femelle, puis la tendance màle. II en arait conclu que, si la fécondation se produit au début de la période de maturité de l'orule, on a un produit femelle, et que, si elle est tardive, on a un produit màle. Entre ces deux états, où le sexe serait nettement déterminé, se placerait une phase, où il serait, au contraire, indécis et labile; c'est ce que Thury appelle le moment de vire. A l'époque où il formulait ces idées (1863), on n'arait d'ailleurs aucune notion précise, sur la structure des ovules, ni sur les phénomènes de leur maturation.

En vertu de cette théorie, Thury conseillait aux éleveurs, pour obtenir des femelles, de faire féconder les mères aux débuts des chaleurs, et, au contraire, à la fin, pour avoir des màles. Les premiers essais faits, en Suisse et en France, eurent des résultats conformes aux prévisions et donnèrent beaucoup de prestige à cette loi. Mais ultérieurement les statistiques offrirent autant de faits en sens contraire et elle fut abandonnée. Nous voyons là, en passant, un exemple significatif de la vanité des statistiques fondées sur un nombre de cas insuffisant (quoique pouvant être très élevé). Des coïncidences peurent donner l'illusion de la rérification d'une idée a priori.

Dans la conception de Thury, la détermination du sexe dépend de l'ovule seul. Bugnion, qui a publié récemment une revue courte, mais substantielle, des recherches relatives au problème qui nous occupe, se demande si l'imperfection de la concordance des résultats avec les prévisions ne tient pas à cette unilatéralité -de la ioi. Si l'on admet que la tendance de l'ovule à évoluer en un embryon femelle ou mâle varie avec son degré 
de maturation, pourquoi le spermatozoïde ne passerait-il pas par des phases analogues? Le sexe de l'ceuf dépendrait finalement de la résultante des tendances des deux gamètes; au moment de la fécondation. Il serait fonction à la fois, et de l'àge de l'ovule, et de celui du spermatozoïde. Bugnion essaie ainsi de corriger ce que l'idée de Thury avait de trop unilatéral; mais la base physiologique reste aussi précaire et, si l'àge de l'ovule peut, à la rigueur, être apprécié, cela devient pratiquement impossible pour celui du spermatozoïde.

$\mathrm{Au}$ reste, on a formulé beaucoup de règles, tout aussi empiriques, reposant sur la comparaison des deux gamètes et faisant entrer en ligne de compte l'àge des deux progéniteurs, leur force relative, etc., tous éléments qui ne correspondent pas à des propriétés tangibles de l'ovule ou du spermatozoïde.'

Théorie de la transmission croisée du sexe. - A ces diverses hypothèses, on en a généralement ajouté une autre, qu'on peut appeler celle de la transmission croisée du sexe. Le gamète le plus fort est l'élément déterminateur et transmet le sexe opposé au sien propre.

On justifie cette loi d'une façon métaphysique. Si le sexe transmis était celui du gamète le plus fort, le résultat serait une rupture de plus en plus grande de l'équilibre numérique entre les sexes, contrairement au principe d'autorégulation, tel que nous l'arons ru plus haut, formulé par Düsing. Mais ce sont là autant d'affirmations arbitraires et dépourrues de sens biologique véritable.

La parthénogénèse arrhénotoque des Rotifères et des Abeilles serait, il est vrai, un fait à l'appui de l'hypothèse; ici, en effet, le sexe transmis par l'ovule non fécondé est le sexe mâle, c'est-à-dire le sexe opposé; 
mais on ne peut considérer ces cas comme l'indice d'une loi générale, car elle serait infirmée par l'existence de la parthénogénèse thélytoque, beaucoup plus répandue.

En réalité, toutes les prétendues règles de détermination du sexe basées sur les rapports d'àge des progéniteurs, ou sur leur force relative, ne sont que des conceptions imprécises, sans valeur scientifique réelle. Si l'on cite des statistiques à l'appui, elles sont tou¡ours trop limitées et on pourrait en réunir qui conduiraient aux conclusions inverses. L'histoire même de la loi de Thury le proure.

De plus, c'est surtout pour les Mammifères que l'on a voulu formuler des règles; or, le nombre des orules qui se développent en embryons y est infime, et d'une façon absolue, et par rapport au total que renferme l'ovaire. Ces animaux offrent donc, a priori, des difficultés à peu près insurmontables à une étude scientifique de la détermination du sexe.

Expériences récentes sur les Batraciens. - Chez d'autres animaux tels que les Batraciens, cette objection disparaît et $\mathrm{R}$. Hertwig, dans ces dernières années, a cherché, sur les Grenouilles, à soumettre à une base expérimentale précise les idées de Thury.

A l'épo de la reproduction, les deux sexes s'accouplent, le màle enserre la femelle, en arrière de la région scapulaire, à l'aide de ses membres antérieurs, pendant plusieurs heures; sous cette pression, les œufs mûrs se détachent de l'ovaire et tombent dans l'oriducte, où ils cheminent lentement vers l'extérieur; ils se recouvrent en chemin d'une couche muqueuse, qui gonflera dans l'eau, une fois les œufs arrivés au dehors. C'est seulement au moment où les ovules sortent du corps de la femelle qu'ils sont fécondés par le sperme du mâle. Si on sépare un couple peu de temps après 
le début de l'accouplement, les ovules restent dans l'oviducte de la femelle et ne sont pas pondus. On a donc là un moyen d'avoir des orules mủrs, détachés de l'ovaire physiologiquement, à un moment connu et dont la ponte est différée. En prélevant, dans l'oviducte, des lots de ces ovules, au bout de durées graduées, et en les fécondant artificiellement par du sperme d'un mème mâle, on pourra roir, d'après le pourcentage des mâles et des femelles issus de ces lots successifs, si l'àge des orules, au moment de la fécondation, a une influence sur le sexe ${ }^{1}$. Il y a donc là un moyen assez précis de rérifier la conception de Thury.

R. Hertwig a basé, sur ces considérations, des recherches variées patientes, qu'il a poursuivies, depuis plusieurs années, avec ses élèves. Les résultats n'en sont pas très nets. Il semble cependant qu'en retardant la fécondation, on augmente la proportion des mâles, conformément à l'idée de Thury. Mais les chiffres sont insuffisants, surtout si l'on n'oublie pas que, dans chaque expérience, il y a une mortalité considérable de tètards, dont le sexe n'a pu ètre connu. Il est malaisé, d'autre part, d'opérer avec des spermatozoïdes absolument comparables. Enfin, on se heurte à la difficulté de reconnaître le sexe des jeunes Grenouilles, de façon absolument sûre, avant l'âge de deux ans. La Grenouille, favorable pour opérer expéri-

1. Le procédé précédent donne facilement des ovules trop mûrs. On peut, par divers artifices, avoir aussi des ovules prématurés : en ligaturant une femelle dans la région axillaire, un peu avant la période normale de reproduction, ou en accouplant des femelles dans les mémes conditions d'époque, avec des mâles provenant des régions plus chaudes et đẻja en reproduction. Nous avons vu plus haut que le réflexe de l'embrassement de la femelle par le mâle est déterminé chez ce dernier par des hormones sécrétées par la glande génitale à l'époque du rut. 
mentalement la fécondation, ne l'est pas pour une diagnose précoce du sexe.

Je rapprocherai de ces expériences, celles que Miss Helen King a faites, dans ces dernières années, sur les Crapauds. Elle n'a pu mettre en évidence aucune influence de la température ou de la nutrition, au cours du déreloppement, sur le sexe. Elle a cherché alors si l'on ne pourrait agir sur celui-ci, en faisant varier les conditions extérieures, au moment de la fécondation.

Des direrses tentatives qu'elle a faites, une seule a donné des résultats assez nets : c'est la déshydratation des orules, opérée, soit en les traitant par des solutions hypertoniques (sel marin 2,5\%), soit en les fécondant à sec et ne les remettant dans l'eau qu'au bout de quelques heures. Dans ces conditions, Miss King a obtenu 60 à $72 \%$ de femelles, alors que des lots d'orules prélevés, en même temps, dans la mème femelle et fécondés, en même temps, avec le même sperme, mais dans des conditions normales, n'en donnaient que 50 à $53 \%$. Il semble donc que la déshydratation de l'ovule, lors de la fécondation, ait une action réelle et on peut rapprocher de cela une pratique de certains pisciculteurs, qui, en fécondant à sec les œufs de Truites, disent obtenir une plus forte propertion d'individus femelles. 


\section{CHAPITRE XIV}

\section{LES $\leadsto R O M O S O M E S$ DES GAMÈTES ET LA DÉTERMINATION DU SEXE}

Le chromoso:ne accessoire dans la spermatogénèse de divers Insectes. - Dimorphisme nucléaire des spermatides. - Théorie d'Ed. Wilson. - Cas divers. - Généralisation. - Objections et restrictions de fait et de principe. - Valeur qualitative ou quantitative des chromosomes spéciaux. - Leur présence dans des animaux hermaphrodites. - Cas des Pucerons.

L'étude cytologique de la spermatogénèse, de l'ovogénèse et de la fécondation ${ }^{1}$, poursuivie, depuis vingt ans, d'une façon si minutieuse, chez un grand nombre d'animaux et de plantes, a fouillé, dans leurs moindres détails, les divers éléments constituant les noyaux des gamètes et surtout les chromosomes. Or, chez dirers Insectes et ensuite, dans d'autres groupes du règne animal, on a mis ainsi en évidence un dimorphisme nucléaire des spermatozoïdes et par suite des œufs fécondés par eux. Ce dimorphisme nucléaire se retrouverait, chez ces mèmes espèces, dans les tissus somatiques du mâle et de ia femelle. Si des faits de cet ordre étaient établis d'une façon indiscutable et générale, ils constitueraient un argument matériel important, en faveur de la détermination syngamique du sexe. On conçoit aisément l'intérèt qu'ils ont éreillé et les nombreuses recherches qu'ils ont provoquées.

1. Cf. ch. III, p. 24 et seq. 
Voyons d'abord, sur un cas particulier bien typique, comment les faits se présentent. Je l'emprunte à des recherches très soignées de de Sinéty sur la spermatogénèse des Orthoptères et en particulier des Phasmides, ces insectes aux formes bizarres, dont l'aspect rappelle à s'y méprendre, chez les uns (Bacillus), une brindille de bois mort, chez d'autres (Phyllium), une feuille desséchée, etc...

Dans les dernières divisions des spermatogonies ${ }^{1}$, la figure caryocinétique, à côté de nombreux chromosomes semblables entre eux, en montre un de beaucoup plus grande taille que nous désignerons par X (fig. 29). Tandis que, dans les spermatocytes de premier ordre, ou auxocytes (issus des spermatogonies), tous les chromosomes ont disparu dans le noyau au repos, l'élément X a persisté. Il a également une allure toute particulière, dans les deux divisions cellulaires que ront subir les spermatocytes et que nous arons appelées divisions maturatives, ou méiotiques. (le nombre des chromosomes y est réduit de moitié). A la première de ces divisions, en effet, - celle des auxocytes en préspermatides, - l'élément $X$ ne se divise pas, comme les autres chromosomes, mais passe, tout entier, dans une des deux préspermatides formées. A la dixision suivante, celle des préspermatides en spermatides, - il se divisera, arec les autres chromosomes, dans les préspermatides où il existe. Il en résulte que, sur les quatre spermatides (et par suite sur les quatre spermatozoïdes) issues d'un même spermatocyte, deux possèdent l'élément $\mathrm{X}$ et deux autres ne le possèdent pas. Il y a donc, relativement à l'élément $\mathrm{X}$, deux catégories de spermatozoïdes produites en nombre égaux, l'une qui le possède, l'autre qui ne le possède pas.

1. Se reporter, pour la terminologie, au chapitre II. 
Cet exemple si net, comme le montre la figure 29, n'est pas le.premier qui ait été connu. Déjà, en 1891, Henking arait signalé un fait de ce genre dans la spermatogénèse d'une Punaise (Pyrrhocoris aptera).

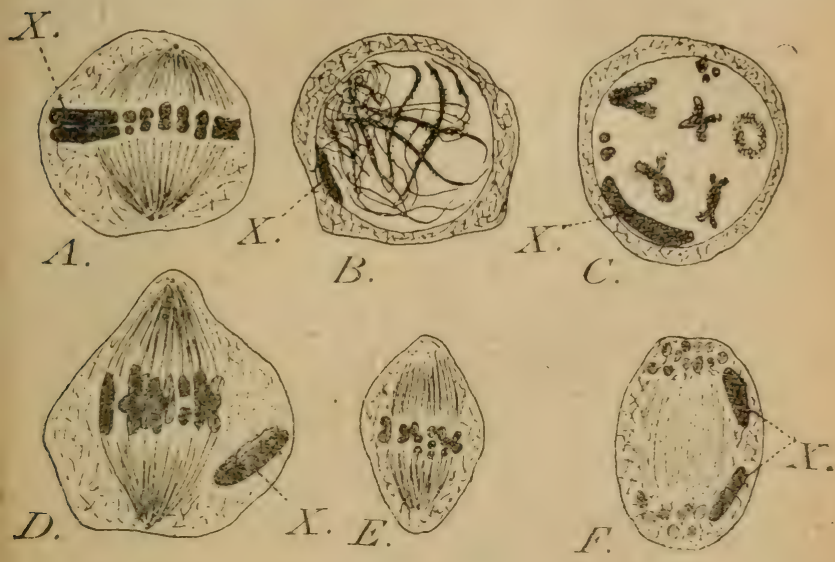

Fig. 29. - Phase míiotique de la spermalogénèse d'une Sauterelle (Orphanic denticauda). montrant le chromosome $X$ (d'après de Sinéty).

$A$, division d'une spermatogonie (stade de la plaque équitoriale vu de profil); $B$, Auxocyle au stale synąsis (houquet; lélément $X$ a persisté à part); $C$, Prélude de la division d'une auxocyte (formation des chromosomes; on distingue tnujours.$X_{\text {; }}$ D. division dune auxoegte (plaque équatoriale de proht; milose hétenotupique. Le chromosome $X$ ne s'est pas divisé et est rejeté tout entier vers un des pôles); $E$, division d'une préspermatide ( $2^{\bullet}$ division mé.otique; viaque équatoriale de profil) ne renfermant pas l'élément $\boldsymbol{X}$; $F$, même stiude, vous une préspermatide renfermant l'élément $\boldsymbol{X}$.

On en arait tronvé ensuite une série d'autres et on avait appelé l'élément $\mathrm{X}$, chromosome accessoire. On le rencontrail surtout chez des Insectes de divers ordres et particulièrement chez les Ilémiptères (Punaises). En 1902, un auteur américain, Mac Clung suggéra que ce dimorphisme chromosomique des spermatozoïdes pouvait être en rapport avec le sexe. 
Après la fécondation, il devait y aroir en effet, en nombres égaux, deux catégories a'œufs, différant entre elles par la composition chromatique du noyau, suivant qu'ils avaient été fécondés par un spermatozoïde possédant ou ne possédant pas le chromosome X. Or, l'espèce présente, en nombres égaux, deux catégrories d'individus, les màles et les femelles. Il était naturel de supposer que les deux faits fussent en comnexion et que le sexe dépendit de la présence ou de l'absence de l'élément $\mathrm{X}$ dans l'œuf. Mais c'est surtout Ed. Wilson qui, dans une série de mémoires publiés depuis 1905, a développé cette idée, lui a donné sa forme précise et y a rattaché les cas, très divergents les uns des autres, qui étaient progressivement mis à jour par une véritable légrion de chercheurs (Sutton, Mac Clung, Paulmier, Montgomery, miss Sterens, Gulick, etc...). Elle ressort nettement des schémas ci-contre.

Le cas le plus simple est celui que présentent divers Insectes Hémiptères (notamment dans les genres Prntenor, Anasa, Alydus, etc...). Il y a, conformément à ce qui a été dit plus haut, deux catésories de spermatozoïdes, se distinguant par l'élément $\mathrm{X}$, et ayant en commun un stock de chromosomes, dont nous désignerons le nombre par $n$.

Elles renferment respectivement $n$ et $n+\mathrm{X}$ chromosomes. L'étude de l'ovogénèse a permis, d'autre part, d'y retrouver l'élément $\mathrm{X}$; mais il existe, après expulsion des globules polaires, dans tous les ovules, qui, par suite, ont tous la composition chromosomique $n+\mathrm{X}$.

La fécondation donne donc, en nombres égaux, les combinaisons :

$$
\begin{array}{cc}
\text { Orule. } & \text { Spermatozoide. } \\
(n+\mathrm{X})+\mathrm{Cur}^{-} & =2 n+\mathrm{X} \\
(n-\mathrm{X})+(n+\mathrm{X}) & =2 n+2 \mathrm{X}
\end{array}
$$


S'il en est ainsi, on doit trouver, pour les tissus somatiques, deux catégories d'individus ayant respectivement $(2 n+1)$ et $(2 n+2)$ chromosomes et c'est ce qu'effectivement Wilson a constaté. Les femelles

Ovule Spermatozoide OEuf Gamètes

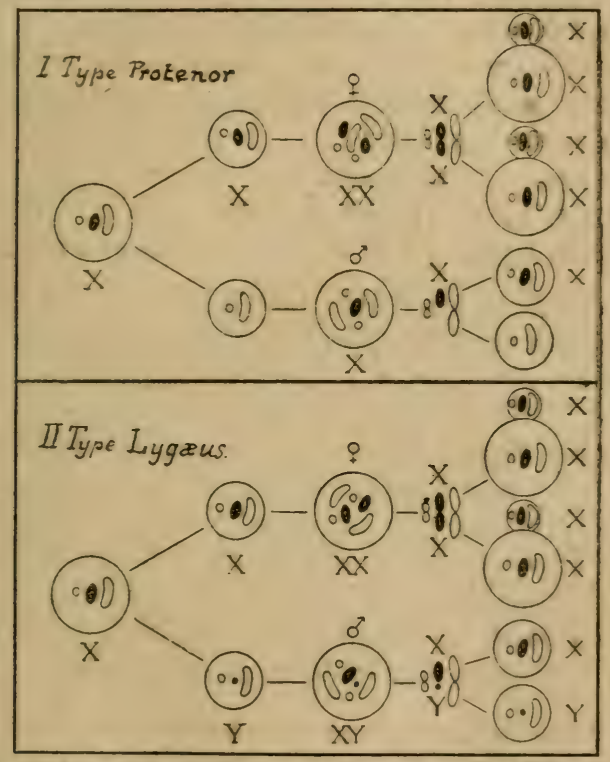

Fic. 30. - Schéma de la distribution des chromosomes $X$ et $Y$ dans les gamètes et l'œuf fécondé (d'après E. Wilson).

ont un nombre de chromosomes pair, les mâles un nombre impair et inférieur d'une unité. Les femelles résulteraient donc des œufs fécondés par les spermatozoïdes possédant i élément $X$. Cet élément déterminerait donc : par sa présence, le sexe femelle, par son absence, le sexe mâle ${ }^{1}$.

1. Pour qu'une observation de cet ordre ne laisse rien à 
Un cas déjai plus complexe est celui qu'ont montré d'autres Hẻmiptères (Lygrus, Carnus, Euschistus, ctc...) ou Coléoptères (Tenebrio). Le dimorphisme des spermatozoïdes consiste en ce qu'une moitié d'entre eux renferme un chromosome spécial $\mathrm{x}$. assez gros et l'autre moitié, un élément correspondant, très petit, que nous désignerons. arec Wilson, par $\mathrm{Y}$; tous les ovules sont semblables entre eux et renferment un élément $\mathrm{X}$. La fécondation réalise alors les deux combinaisons:

$$
\begin{aligned}
& \text { Ovale. } \\
& \text { Spermatozoide. } \\
& \text { Geur. } \\
& (n+\mathrm{X})+(n+\mathrm{X})=2 n+2 \mathrm{X} \\
& (n+\mathrm{X})+(n+\mathrm{Y})=2 n+(\mathrm{X}+\mathrm{Y}) \text { mâles }^{1}
\end{aligned}
$$

Mais ces deux modalités sont loin d'épuiser la série des faits. Au fur et à mesure que des lypes noureaux ont été étudiés, on a décrit des dispro-itions nourelles et distinctes: ici, les deux catécrories de spermatozö̈des diffèrent par tout un grumpe de chromosomes (que l'on considère comme la monnaie, en quelque sorte, du chromosome X); là, par la taille de certains chromosomes (comme dans le cas simple de Lygæus rappelé ci-dessus). Je ne puis songer à entrer ici dans ces détails cytologiques?. Le principe de leur interprétation reste toujours le mème.

désirer, il faut qu'on ait pu compter les chrnmosomes à toittes les étapes : dans les tissus somatiques de l'organisme, dans les spermatogonies et dans les ovogonies, dans la phase méiotique de l'orogénèse et de la spermatogénèse, après li fécondation et dans la segmentation de l'œuf. Pareille série n'a été faite à l'heure actuelle que dans des cas exceptionneliement favorables, par exemple chez divers Hẻmiptères (Protenor, Anasa, Archimerus) par les recherches d'E. Wilson et de sna élève Murrill, et chez un Ver Nématode (Strongylus paradoxus) par A. tiulick.

1. Le premier cas, d'après Wilson, dériverait de celui-ci par l'atrophie progressive de $\mathbf{Y}$, jusqu'à disparition complète.

2. De cette diversité est née aussi une variété très grande dans les dénominations données aux chromosomes différentiels : 
On a cherché ainsi à généraliser les premiers faits observés ${ }^{1}$ : le sexe serait déterminé partout par l'une ou l'autre de deux combinaisons de chromosomes, qui se réalisent lors de la fécondation, par suite du dimorphisme des spermatozoüdes. Si l'exactitude de cette conception était démontrée, on voit que la détermination du sexe serait essentiellement syngamique.

Tous les faits publiés n'offrent pas les mèmes garanties d'exactitude. Les plus anciennement signalés sont, d'une manière générale, les plus nets. Ils ont été constatés, sans suggestion d'aucune sorte et, s'ils n'araient pas été nets, ils auraient passé inapercus. La nature véritable du ou des corps $X$, leur équiralence aux autres chromosomes, restent, il est rrai, sujets à discussion. Mais nous nous bornons ici à constater que, du fait de leur existence, ils introduisent un dimorphisme dans les spermatozoïdes.

Mais quand l'idée de leur rappurt arec le sexe eut été nettement formulée, les chercheurs ontpu parfois se laisser suggestionner par le désir de les retrouver. Il ne faut pas perdre de rue que les figures nucléaires de la sjermatogénèse sont très petites et généralement très compliquées, à cause du graud nombre des chromusumes; qu'on n'assiste pas effectirement à la transfurmation des stades les uns dans les autres, mais qu on est réduit à mettre hypothétiquement en série, d'après des analogies, des figures

chromosome accessoire, hétérochromosome, idiochromosomes, allosomes, etc. Nuus nous en tēnons ici a l'appellatinn $\mathbf{X}$ et $\mathrm{Y}$, qui ne présume rien sur la signification réelle de ces eléments.

1. On a décrit anjourd'hui le dimorphisme clummosomique des epermatozoildes. chez des Insectes appartenant à la plupart des ordres (Ilenil,tirs, Orthopteres, Cinleopteres, Diplieres, etc.) chez des Myriipodos, chez des Nématodes, chez des Oursins, rhez des Oiseaux, des Mammifères, etc.

16. 
observées côte à côte. Dans ces conditions, l'analyse des figures est, dans bien des cas, sujette à beaucoup d'inexactitude, et un observateur aussi éminent que Ed. Wilson a, lui-même, reconnu, par exemple, en 1912, l'inexactitude d'interprétations de chromosomes, qu'il avaient affirmées antérieurement. On a cherché si la spermatogénèse de l'homme présentait des chromosomes $X$, et récemment on a cru les trouver; mais il est significatif que les corps décrits comme tels, par trois observateurs, cytologistes très qualifiés ${ }^{1}$, sont tout à fait discordants. Il y a donc des réserves à faire, au moins sur une partie des faits annoncés.

Pour ceux mèmes qui sont bien exacts, quelle est leur portée véritable? Beaucoup d'auteurs n'ont pas hésité à voir, dans les chromosomes $X$, les éléments déterminateurs directs du sexe, le siège en quelque sorte de la sexualité femelle. Ils leur attribuent, à cet égard, une valeur qualitative.

Cette conception s'est fait d'autant plus naturellement jour, qu'elle rentre dans l'ensemble de celles qui découlent du weismannisme et plus récemment du mendélisme. Bon nombre de cytologistes admettent l'individualité, la permanence et la différenciation qualitative des chromosomes en général, ils y localisent d'une façon absolue le siège de l'hérédité 2 . Chaque chromosome serait, pour eux, le support d'une partie des propriétés, des caractères de l'organisme. On n'éproure alors aucune peine à croire qu'à l'un d'eux corresponde le sexe.

Mais l'autonomie des chromosomes, malgré les adhésions nombreuses et autorisées qu'elle a reçues, est loin d'être un fait démontré. Le serait-elle, la localisation des propriétés héréditaires, en général,

1. F. Guyer, H. Winiwarter et S. Gutherz.

2. Voir chapitre III, p. 33. 
dans le noyau, sur la chromatine et, plus encore, celle de propriétés particulières sur telle ou telle particule chromatique, est une conception des plus discutables.

L'organisme est un tout indivisible physiologiquement. La sexualité est une fonction de ce tout; ainsi que le dit Montgomery, il est aussi inrraisemblable d'en rapporter le siège à une parcelle spéciale de la celluie, que de rapporter à un autre chromosome particulier le substratum de la fonction d'excrétion ou de locomotion; et, suivant l'heureuse expression de Guyer, il ne faut pas chercher à localiser, dans les substances figurées initiales de l'organisme, des caractères spéciaux ultérieurs, pas plus que la farine ou la la levure ne portent en elles-mèmes de déterminants spécifiques des diverses formes de pains.

Abstraction faite de cette objection générale, il y a des difficultés particulières, non moindres, ainsi que l'a montré notamment E. Wilson, à attribuer aux éléments $X$ une valeur qualitative pour la détermination du sexe. Mais ne pourrait-on pas, tout au moins, leur en accorder une de nature quantitative? Beaucoup de biologistes sont tentés de chercher la différence essentielle $e_{\iota}^{t}$ primitive entre les sexes, dans l'intensité plus ou moins grande des échanges à l'intérieur des tissus; l'intensité de ce métabolisme est peut-être fonction de la quantité de chromatine contenue dans le noyau des cellules. Le dimorphisme constaté montre un excès de chromatine chez la femelle. Il ne serait donc pas invraisemblabled'imaginer une relation, au moins indirecte, entre cet excès de chromatine et l'activité métabolique plus grande lu sexe femelle. Dans une récente (1911) et très iumineuse revue des faits, Ed. Wilson s'exprime arec beaucoup de prudence : "Je regarde, dit-il, les chromosomes $X$ comme un anneau - probablement 
essentiel, - dans une chaine de facteurs, gràce auxquels le sexe est déterminé et transmis, et, comme ce sont les plus accessibles et les plus risibles de ces facteurs, nous devons, dans notre analyse des faits, leur accorder toute notre attention. " C'est, comme on voit, ne chercher entre eux et le sexe que des relations très indirectes.

Dircers faits sont de nature à nous inciter à la prudence. Ainsi, on a retrouvé le dimorphisme des spermatozoides chez des animaux hermaphrodites ${ }^{1}$, où il ne peut plus être question de détermination du sexe. Un a admis alors que, des deux catégories de spermatozoïdes, une seule est fonctionnelle. C'est là une conclusion très commode, mais très hardie et qui demandriait à être plus sérieusement étayée qu'eile ne l'a citr, au moins dans certains cas. Elle ne serait pas renue a Iesprit, s'il n'arait pas fallu faire cadrer les faits avec la théorie.

Un paradoxe du même genre s'est rencontré chez les Pucerons, où tous les œufs fécondés deriennent des femielles et oú, cependant, il y a dimorphisme de spermatozoïdes. Ici encore, on a conclu, avec d'assez bonnes rasons d'ailleurs, que l'une des deux catégories diléments mâles arortait toujours, de sorte que, scule, ia combinaison correspondant au seye femelle était réalisée.

Erfin, dans certains cas, - notamment pour les culs males des Pucerons (Phylloxera, Aphis), - les éléments $I$ ne se différencient que lorsque les œufs sont déji caractérisés comme derant donner des málcs. Cela est de nature à faire penser que les chruilusones $X$ peurent ne pas être, au moins dans certa.us cas, un agent causal dans la détermination du sese, mais qu'ils sont seulement un épiphéno-

1. Ascaris nigrovenosa, des Mollusques Ptéropodes et Pulmonés. 
mine, une manifestation concomitante, presque un effet de cètte détermination.

Si l'on rapproche toutes ces considérations, on doit conclure que le dimorphisme nucléaire des spermatozoïdes (ou des ovules) n'a pás, pour la ditermination du sexe, une signification aussi simple qu'on a pu le croire au premier abord. Tous les faits signalés ne présentent pas les mêmes garanties; mais il en est qui, en eux-mêmes et comme interprétation, paraissent indiscutables. Il y a donc là une caténorie de données qui sont de nature à renforcer l'hypothèse d'une détermination syngamique du sexe.

Elles ont reçu, à leur apparition, un accueil d'autant plus farorable, qu'elles semblaient apporter une vérification matérielle d'une autre conccplion de la détermination du sexe fondée sur la loi de Mendel. 


\section{CHAPITRE XV}

\section{LE SEXE CONSIDÉRÉ COMME CARACTĖRE MENDÉLIEN}

Exposé sommaire des lois de Mendel. - Les deux sexes considérés comme formant un couple de gènes mendéliens. Expériences de Correns sur la Bryone. - Interprétation de Correns et de Bateson. - Caractères transmis corrélativement à un sexe (sex-limited inheritance) : expériences sur un Papillon (Abraxas grossulariata). - Mendélisme et polymorphisme sexuel des Papillons.

Conclusions générales relatives au problème de la détermination du sexe.

Chez les espèces gonochoriques, les deux sexes constituent un couple de propriétés dont l'une exclut l'autre, chez un indiridu. Si on les considère comme transmis héréditairement, ils obéissent donc, de façon typique, à la forme d'hérédité dite alternative ; c'est l'un ou l'autre qui est hérité et non une propriété moyenne entre eux. Or, les particularités, qui se comportent ainsi, sont celles auxquelles s'appliquent, d'une façon générale, le plus nettement les lois de Mendel. Il était donc naturel que l'école néomendélienne s'efforcât d'expliquer la détermination du sexe par le mécanisme de ces lois. Mendel, lui-même, avait déjả fait une tentative en ce sens.

§ 1. - Exposé sommaire des règles de Mendel. - Rappelons, d'une façon brève et schématique, les 
points fondamentaux relatifs à l'hérédité mendélienne. Supposons deux variétés d'une espèce, différant par un caractère. Croisons-les. Nous obtenons une génération filiale (que nous noterons $F_{1}$ ), dite hybride, participant héréditairement des propriétés des parents $P$. Croisons à noureau ces hybrides entre eux; nous formons ainsi une seconde génération $F_{2}$. L'observation montre, dans des cas nombreux, qu'un quart environ des individus $F_{\mathrm{a}}$ est du type pur d'une des rariétés initiales parentes; un autre quart est identique à l'autre parent; enfin les deux derniers quarts sont de nature hybride, comme la génération $F_{1}$. On s'explique aisément ces résultats, en admettant que la constitution hybride de la génération $F_{1}$ ne s'étend pas aux gamètes, mais que ceux-ci sont purs, c'est-à-dire identiques à ceux de l'une ou de l'autre des formes parentes. La combinaison de ces formes, réalisée dans le soma des hybrides, s'est dissociée dans les gamètes. Par raison de symétrie, on doit admettre qu'il y a, en nombres égaux, des gamètes de chacun des types parents. Si nous désignons ces types par $D$ et $R$, les somas des hybrides $F_{1}$ seront mixtes $D R$, mais leurs gamètes seront $D$ ou $P$. Dès lors, dans la fécondation des $F_{1}$ entre eux, pour produire les $F_{2}$, il se produira, en nombre égaux, toutes les combinaisons possibles de gamètes $D$ et $R$ avec $D$ et $R$, c'est-à-dire $D D, D R, R D, R R$, ou. $D D+2 D R+R R$.

Les individus $F_{2}$, qui reproduisent les parents purs. résultent de la fusion de deux gamètes de mème type. ( $D D$ ou $R R$ ). On les appelle homozygotes. Il y a $25 \%$ de chacun des deux types. Les hybrides, au contraire, résultent de la fusion de deux gamètes de types différents; on les appeile hétérozygotes $(D R)$. Il y en a. $50 \%$.

La génération $F_{1}$ ne renferme que des hybrides, ou 
hétérozygotes, et deux cas peurent se présenter : ou bien, en apparence, ils ressemblent à un des parents $D$, dont nous disons que le caractère est dominant (D), ou bien ils ont un faciès intermédiaire entre les deux parents. Dans les deux cas, la générâtion $F_{1}$ ne renferme aucun individu ressemblant à l'autre parent $R$. Ce type disparait à la génération $F_{1}$, pour reparaitre à la suivante $F_{2}$, chez un quart des individus. On dit qu'il est récessif $(R)$; il est masqué, quand il se combine arec le type dominant, pour forner un hybride.

Examinors encore ce qui arrive quanl. an licu de croiser les individus $F_{1}$ entre eux, on cruise un $F_{1}$, arec l'un des parents $D$ ou $R$. Les $F_{1}$ (hydrides $D R$ ) ont des gametes, $D$ ou $R$, qui vont se rumbirier (dans le cas du parent (D) arec des gamètes, tous du type $D$. Il y aura dunc nombres égaux de combinaisurs $D D$ et $D R$, sur $50 \%$ d indiridus homozyeutes du type $D$ et $50 \%$ d'hétéroygotes $D R$. Si le croisement était fait avec le parent $R$, on aurait, de mème, $50 \%$ d'hétérozygotes $D R$ et $50 \%$ d'homozygutes $R R$.

§ 2. - Application au problème des sexes. Considérons maintenant les sexes comme furmant, dans une espèce, un couple de caractires $D . R$, ou de gènes, suirant le terme introduit par Johannsen. Désignons le gène mále par le signe ơ, le gène femelle par ㅇ․

Dans la grérínlité des espèces, il y a nombres rigaux, $50 \%$, de $1 . . .$. et de femelles. Les résullats du croisement naiurel des individus est donc nunuriquement le même que celui du croisement des $F_{1}$ hétéroz!gotes, arec un type parent homozygote 'P. On expliquerait donc l'égalité numérique des sexes, en suppusant que l'un d'eux est homozygote par rapport au gène de la sexualité, l'autre étant hétéruzygote; gar exemple, en supposant que les mâles sont homo- 
zygotes $\left(\sigma^{\Upsilon} \sigma^{\top}\right)$ et les femelles hétérozygotes $\left(\sigma^{\top} Q\right)$, à la condition de supposer, en outre, que le gène $q$ soit dominant par rapport au gène $\sigma^{x}$. Il suftit de se reporter aux raisonnements précédents. Le croisement mâle $\times$ femelle $\left(\sigma^{\top} \sigma^{\top}\right) \times\left(\sigma^{\top} \%\right.$, doit donner $50 \%$ de $\sigma^{x} \sigma^{\Upsilon}$ et $50 \%\left(\sigma^{x} \%\right)$; ces derniers seront des femelles puisque, par hypothèse, le gène $O$ domine le $\sigma^{7}$. On pourrait, d'ailleurs, tout aussi bien supposer, a priori, que $1 \mathcal{E}$ anâle est hétérozygote ơ et la femelle homozygote $\bigcirc_{+}$; mais alor's il faudra admettre que le gène $\sigma^{\Upsilon}$ domine le gène q.

Telle est la forme de l'hypothèse à laqquelle a conduit, pour la sexualité, la conception mendélienne. Elle participe de la nature générale de celle-ci. Elle fait de la sexualité une entité distincte, suplerposée en quelque sorte au reste de l'organisme. Si on ne lui accorde que la valeur d'un pur symbole, clle fournit évidemment une représentation de l'cirlité numérique des sexes. Si on y veut roir darankage, il convient de so demander, si l'on est en droil de faire ainsi, de la sexuahlé, une entité indépendante, superposée au reste de l'organisme; mais c'est l'objection qui se dressic deránt toute la doctrine mendéienne.

En lunt ras. celte conception entraine arec elle la déterminaliurs s!llgamique du sexe, de façon éridente.

Expérienres de Correns sur la Bryone. - La théorie monlílieme du sexe, dans la forme précedente, a été formulé: far le botaniste Correns et il est intéressint d'examiner, d'une façon un pren détaillée, le cas snr lempul il l'a basée. Il a expérintenl's sur une plante bicn connue de la famille des cincurbitacées, la Bryone diongue (Bryonia dioüca). Charpue pied de cette plante ue renferme que des thenr: dian mème sexe. Cependint, chez les pieds femelies, on troure parfois des rudiments d'étamines dans les fleurs. 
L'unisexualité n'est pas une propriété absolue comme chez les animaux. Une plante roisine, la Bryone blanche $(B$. alba), a, au contraire, des fleurs tou-. jours hermaphrodites. Dans ce qui va suivre, nous désignerons ces deux plantes par les leltres $d$ et $a$. Les gamètes de $B$. dioïca (d) seront çonsidérés comme tous porteurs de la propriété (ou gène) dioïcité; ils doivent avoir en outre une sexualité propre, que l'expérience déterminera. Les gamètes de $B$. alba (a) porteront tous le gène monoicité $\sigma^{\prime}$. Il n'y a pas lieu de leur supposer une sexualité propre.

Correns fait les trois expériences suirantes:

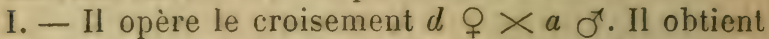
ainsi des graines hybrides qui, semées, donnèrent, toutes, des plantes $F_{1}$ dioïques et femelles (sauf la prèsence de quelques rares fleurs mâles précoces). Il a eu ainsi 587 pieds femelles et 2 pieds màles. Il conclut, de cette première expérience, que le gène dioïcité est dominant sur la monoïcité et, puisque toutes les plantes (sauf 2 sur 589) sont femelles, c'est que tous les gamètes femelles employés ont la terdance femelle.

II. - Il fait ensuite le croisement normal des deux sexes de la Bryone dioïque $d \% \times d \sigma^{x}$. Il obtient nombres égaux de pieds mâles et de pieds femelles.

III. - Il croise enfin $a q x d \sigma^{x}$. Il obtient des plantes toutes diö̈ques. Cela confirme l'hypothèse que le gène dioïcité est dominant sur monoicité. Mais, rette fois, $50 \%$ des pieds $\mathrm{F}_{1}$ sont mâles et $50 \%$ sont femelles. Donc parmi les gamètes d $\sigma^{x}$ : une moitié a la tendance $\sigma^{x}$, c'est-à-dire est porteuse du gène $\sigma^{x}$; l'autre moitié a la tendance $O$, c'est-à-dire est porteuse du gène femelle.

Correns conclut donc de cette analyse que, chez la Bryone dioïque, le sexe femelle est homozygote $q$ 우 et le sexe mâle hétérozygote $\sigma^{x}$ 오.

Cela résulte des expériences I et III, qui, répétées 
plusieurs fois, avec des pla es d'origines diverses, ont toujours donné le même résultat. L'expérience II montre alors que le gène $\sigma^{x}$ est dominant par rapport au gène femelle.

Ces expériences, surtout au premier abord, sont très convaincantes et cependant elles contiennent une part d'arbitraire très grande dans l'interprétation. On est amené, en effet, à faire certaines conventions qui entraînent toute la suite. Or ces conventions initiales sont souvent purement à la volonté de l'expérimentateur et, arec de l'ingéniosité, on peut expliquer les mêmes résultats par plusieurs hypothèses, dont certaines contradictoires. C'est ainsi que l'un des représentants les plus éminents dii néo-mendélisme. Bateson, a donné, des résultats expérimentaux de Correns, une interprétation inverse. Il considère, en effet, le sexe màle de $B$. dioïca, comme homozygote $\sigma^{x} \sigma^{x}$, et le sexe femelle comme hétérozygote $\sigma^{x} \mathcal{T}$, mais, cette fois, le gène $q$ dominant le gène $\sigma^{x}$. Mais, à la différence de Correns, il suppose que les gamètes de la Bryone blanche ont, eux aussi, un gène de sexualité : les organes femelles de cette plante seraient hétérozygotes $\left(\sigma^{\top} \%\right)$ et les organes mailes, homozygotes, avec tendance femelle ( $ᄋ$ 우), hypothèse assez bizarre, mais logiquement possible. Hoyennant ces conventions; on rend aussi bien compte des faits qu'arec les hypothèses de Correns, comme on s'en convaincra par l'examen du tableau ci-contre (p. 196), où ces deux interprétations sont mises en parallèle 1 .

A la vérité, de nouvelles épreuves pourraient montrer quelle est la plus correcte des deux interprétations, de Bateson ou de Correns. Dans celle de Bate-

1. Pour mettre mieux en évidence les hypothèses de dominance, on a représenté les gènes $o^{*}$ et $\%$, par les lettres $m$ et $t$, la sexualité dominante étant signalée par une majuscule; $h$ indique l'hermaphrodisme ou monoïcité. 


\begin{tabular}{|c|c|c|c|c|c|c|c|c|c|}
\hline $\begin{array}{l}70 \\
0 \\
= \\
= \\
=\end{array}$ & 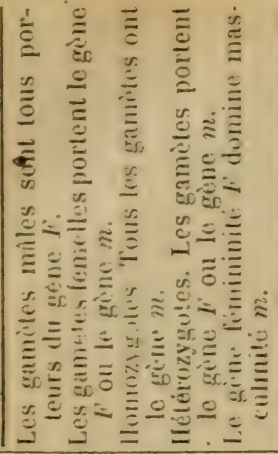 & $\begin{array}{l} \\
\\
0 \\
0 \\
0 \\
0 \\
0\end{array}$ & 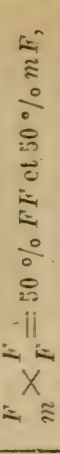 & 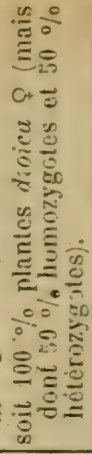 & 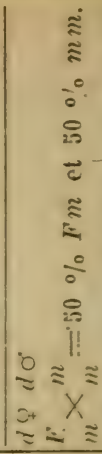 & 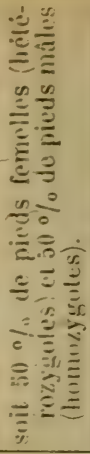 & $\begin{array}{l}0 \\
0 \\
0+ \\
0=\end{array}$ & 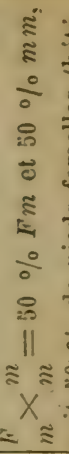 & 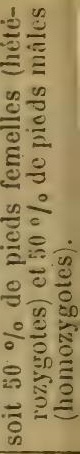 \\
\hline $\begin{array}{l}\sum_{0}^{2} \\
\tilde{E} \\
\tilde{E}\end{array}$ & 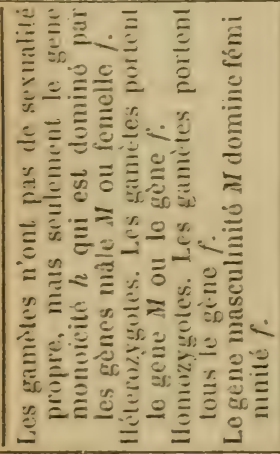 & $\mid \begin{array}{l}0 \\
0 \\
0 \\
0\end{array}$ & $\begin{array}{l}\approx \\
\stackrel{0}{\circ} \\
\stackrel{5}{0} \\
i i\end{array}$ & 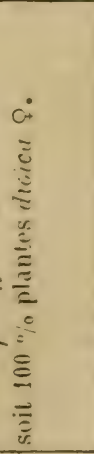 & $\begin{array}{l}2 \\
\therefore \\
0 \\
0 \\
+ \\
+ \\
\vdots \\
0 \\
0 \\
0 \\
0 \\
11\end{array}$ & 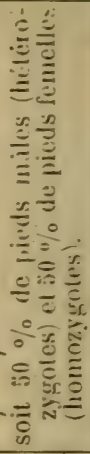 & $\begin{array}{l}0 \\
\approx \\
\mathrm{C}_{+} \\
\approx\end{array}$ & $\begin{array}{l}\approx \\
\approx \\
0 \\
0 \\
0 \\
\approx 0 \\
\overrightarrow{0} \\
\approx \\
\frac{0}{0} \\
0 \\
\approx 0\end{array}$ & 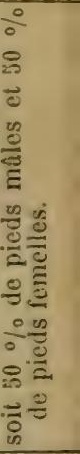 \\
\hline & 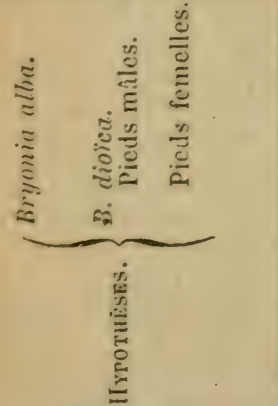 & & 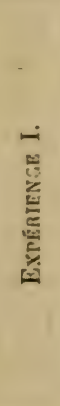 & 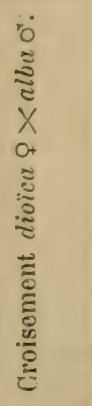 & 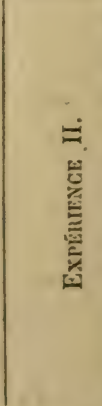 & 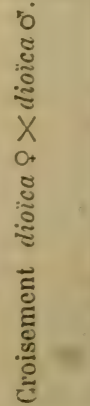 & & $\Xi$ & 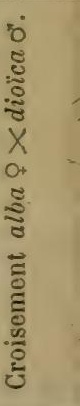 \\
\hline
\end{tabular}


son, les plantes issues de la première expérience doivent ètre de deux natures: les unes hon:ozy rotes $F F$, les autres hétérozygotes $\mathrm{Fm}$. Dans celle de Correns, elles sont toutes de mème nature $f h$. En fícondant ces plantes par du pollen de $B$. dioïca, il est fictile de voir que les hypothèses de Correns font privoir, dans la seconde génération, $50 \%$ de pieds màles et $50 \%$ de pieds fenclles; celle de Bateson, $75 \%$ de pieds femelles $F m$ et $25 \%$ de pieds mâles $m \mathrm{~m}$. Malheureusement. les hybrides $\mathrm{F}_{1}$ de $B$. alba ct $B$. dioíca se sont muntrés stériles et l'on doit s'en tenir aux résultats précédents, qu'expliquent également les deux groupes d'hypothèses.

L'exemple des Bryones montre bien, en tout cas, comnient se pose le problème du sexe, considéré comme caractère mendélien. Les biolog̣istes de l'école néo-mendélienne en ont fait d'assez nombrenses applications, cn expérimentant sur des cas, où certains caractires somatiques sont transmis sulidairement arec un sexe, à l'exclusion de l'autre. C' 'st re que les Anglais appellent sex-limited inheritance. En roici un exemple simple. Les Moutons de race Durset ont des cornes dans les deux sexes; ceux de race Sulfolk n'en ont jamais. En faisant des croisements de ces deux races. on a observé que, chez les hrbrides, les màles sont cormus et que les femelles ne le sont pas. En style néo-mendélien, on dit donc que, dans ce croisement le grène (existence des cornes) est lié à celui du sexe mâle.

Cas diAbraxas grossulariata. - Un Papillon très commun. dont la chenille vit sur le Groseillier, Abiaxas grossulariata, aux ailes blanches tachetées de noir el d'orangé, se montre parfois sous une rariété très paile, dite lacticolor, mais dont on ne rencontre, dans la nature (au moins en Angleterre), que des indı- 
ridus femelles. Par une série de croisements expéri. mentaux appropriés, dans le détail desquels je ne puis entrer, Doncaster a cependant réussi à obtenir les mâles de cette variété lacticolor. Bateson et Punrett ont très ingénieusement montré que l'on se rend compte des résultats de ces croisements, tant pour l'existence des diverses formes que pour les nombres relatifs des individus de chacune d'elles, en faisant les suppositions suivantes : chez ce Papillon, les mâles seraient homozygotes, pour le gène de la sexualité $m m$; les femelles seraient hétérozrgotes, arec dominance du gène féminin $F m^{1}$; la forme typique et sa variété lacticolor correspondraient à une paire de crènes conjugés, le type $G$ dominant la variété $l$; enfin on admettrait qu'il $\mathrm{y}$ a incompatibilité entre les gènes $F_{x}$ et $F$, qui ne pourraient coexister dans un même gamète.

Mais il ne faut pas perdre de vue qu'un pareil symbolisme, si ingénieux soit-il, contient, au départ, un certain nombre de conrentions arbitraires (telles qu'ici l'incompatibilité des gènes $F$ et $G$, par exemple) et que, par cela même, il n'a qu'une valeur relative. Lang a pu, par exemple, représenter le cas de l'A braxas grossulariata, par une autre série de conventions. C'est un symbolisme et l'hypothèse mendé-

1. C'est par analogie avec ce cas que Bateson a proposé d'expliquer celui de la Bryone, par des conventions inverses de celles proposées par Correns. Remarquons qu'il n'est pas nécessaire de supposer, a priori, que tous les organismes se comportent de méme. Morgan a été amené à faire, pour les sexes de la mouche des vinaigreries (Drosophila), des hypothèses inverses de celles de Bateson et coincidant avec celles de Correns.

2. On a, par exemple, essayé de représenter, par des combinaisons appropriées de gènes, liés à ceux de la sexualité, le polymorphisme des femelles de certains Papillıns, tels que Papilio memnon de Java par exemple. Cela a servi de suide à des expériences de croisement, encore en cours, pour obtenir telle ou 
lienne du sexe n'est, en somme, que l'application de l'arithmétique mendélienne au fait de l'égalité numérique des sexes. Ce symbolisme peut donc être un guide utile ${ }^{2}$ pour suggérer certaines expériences. Il peut se rattacher indirectement à la réalité, mais il ne faut pas s'aveugler sur sa valeur absolúte.

C'est ce que l'on perd trop souvent de vue, dans la faveur dont jouit actuellement le néo-mendélisme. En introduisant des hypothèses complémentaires convenablement choisies, sur les relations entre les gènes, ou en imaginant des gènes supplémentaires, on représente bien les résultats constatés, mais on ne les explique pas réritablement. On n'en fait qu'une transcription symbolique plus ou moins compliquée.

Et celle-ci n'a de valeur véritable que si elle permet de prévoir ce que donneront des croisements noureaux en vue desquels elle n'a pas été faite. C'est à ce critérium qu'on jugera si elle correspond rraiment à un élément de la réalité.

Le mendélisme se présente à cet égard, comme autrefois la théorie atomique en chimie. Les adversaires de cette théorie n'y royaient qu'un symbolisme subjectif. La fécondité de ce symbolisme a été la réponse décisive des atomistes. Ainsi s'éprouvera la valeur véritable de la conception mendélienne. Le résultat ne peut pas être considéré comme actuellement acquis.

\$3. - Conclusions générales relatives au déterminisme du sexe. - Nous arons ainsi passé en rerue les divers ordres de faits, actuellement connus, relati-

telle de ces formes. Sans doute, on n'explique pas ainsi l'origine de ce polymorphisme, mais on le conçoit, en dehors de toute idée finaliste, comme celles auxquelles avait abusivement conduit le mimétisme. 
rement à la détermination du sexe. Il ne s'en dégage pas éridemment une solution claire. Rien n'indique, au reste, qu'il doire en exister une unique.

Le sexe des animaux gonochoriques, s'il ne se manifesle parfois quassez tardivement dans le développement, doit ètre fixé de fort bonne heure, et les cas oir un avait cru pouvoir le modifier par l'action de facteurs extérieurs agissant.sur l'embryon, surtout à des stades arancés, semblent bien être illusoires. Les données de fait sont donc contraires à la théorie épigamique. Dans des cas particuliers assez restreints, le sexe est manifestement déterminé dans l'orule, arant la fécondation; mais l'hypothèse de la détermination progamique ne peut, en somme, se réclamer de faits authentiques bien nombreux et elle se heurte à l'invraiscmblance que le spermatozoïde ne jouerait aucun rôle.

C'est done à la détermination syngamique qu'on est ramene frar voie d'exclusion. D'autre part, divers faits positif, encore limités, sont en sa faveur. On doit compter aussi, dans ce sens, ceux qu'a fournis la cytolosie en mettant en éridence un dimorphisme. nucléaire dessperınatozoïdes; mais il convient actuellement de ne pas attribuer à ce dimorphisme une géníralité trop grande, ni un sens trop précis. Enfin, la détermination syngamique du sexe cadre fort bien avec la conception mendélienne de l'hérédité, en faveur actuellement.

Si l'on est amené ainsi à admettre que le sere se délernine au moment de la fécondalion et découle de la constitution particulière de l'cuf, qui cst le résultat de celle-ci, il est prudent de ne pas donner a cette conception une signification trop absulue.

De meme que nous arons ru la manifestation des caractires sexuels secondaires dépentre largement du métaholisme général de l'organisme, de inème le sexe 
proprement dit pourrait lui être intimement lié et cette corrélation doit exister dès les premiers états de l'organisme. Il est donc naturel d'admettre que la constitution particulière de chaque œuf soit un facteur prépondérant, dans la détermination du sexe et que les facteurs extérieurs ne peurent avoir qu'une influence très restreinte. Mais, logiquement, il serait exagéré de nier, d'une façon absolue, leur efficacité et les faits n'imposent nullement de le faire.

Ed. Wilson, qu'on ne peut suspecter en l'occurrence d'exagérer la part des actions extérieures à l'organisme, me semble penser très justement en disant que le sexe dans l'œuf fécondé est à l'état de prédisposition plus ou moins forte, mais non de prédétermination immuable. Cette formule a l'avantage de se concilier arec les cas-limites du gonochorisme et de l'hermaphrodisme, et arec la réapparition accidentelle, plus ou moins rudimentaire, de l'hermaphrodisme, chez les types gonochoriques. Les deux sexes ne doivent pas, chez eux, ètre conçus d'une façon absolue, comme deux polarités incompatibles; il ne s'agit pas, pour l'œuf, de choisir entre une boule noire et une boule blanche, comme le sugreire trop la mentalité mendélienne. Il est plus vraisemblable que les deux possibilités coexistent d'abord virtuellement et le résultat final doit être envisagé comme la prédominance de l'une sur l'autre. Il n'en est pas moins rrai, comme le dit Wilson, que les faits cytologiques et la plupart des faits expérimentaux plaident contre la détermination tardive du sexe par les facteurs externes. Si ceux-ci agissent, c'est en modifiant un mécanisme préexistant dans l'œuf.

C'est à une conception de cet ordre que se ramènent d'ailleurs tous les problèmes généraux de l'embryogénie. La rérité doit être d'apprécier justement la part qui revient à la constitution interne des organismes, 
c’est-à-dire à leurs propriétés héréditaires et au milieu. Si le lamarckisme a eu parfois, de la puissance de ce dernier, une idée exagérée et trop simple, il $y$ a dans les courants dominants de l'heure actuelle une tendance exagérée en sens inverse et elle se fait sentir aussi dans le problème de la détermination du sexe.

On ne saurait surtout oublier que celui-ci est d'une extrême complexité et que nous sommes encore très peu avancés dans son étude. 


\title{
QUATRIEME PARTIE
}

\author{
LA PARTHÉNOGÉNĖSE:
}

\section{CHAPITRE XVI}

\section{LA PARTHÉNOGÉNÈSE NATURELLE}

Définition. - La parthénogénèse est un processus secondaire et non primitif.

Ses degrés : rudimentaire ou complète. - Son extension chez les animaux et chez les végétaux. - Ses divers modes: parthénogénèse accidentelle, facultative; normale, cycliqus (régulière ou irrégulière), constante. - Disparition des mâles: exemples divers.

§1. - Définition. - Chez la généralité des animaux et des végétaux, la fécondation par le gamète màle, spermatozoïde ou anthérozoïde, est la condition nécessaire du développement de l'ovule en uı

1. Le manuscrit de ce volume avait été remis entièrement terminé entre les mains de l'éditeur en avril 1913. Depuis, a paru (juillet 1913), dans la même Collection de Philosophie scientifique, un livre de $\mathbf{Y}$. Delage et M. Goldsmith, sur La Parthénogénèse naturelle et expérimentale. On y trouvera, sur la parthénogénèse

expérimentale, des développements beaucoup plus considérables et plus approfondis que dans les chapitres qui suivent. Les recherches magistrales de Delage dans cє domaine confèrent à ce 
embryon. Fiule d'ètre fécondé et de l'être à un moment assez itroitement déterminé, après l'achèvement de sa maturation, l'ovule devient incapable de l'ètre. sallère el dégénère. Ovule et fécondation semblent donc ètre deux notions indissolublement liẻes.

Et cependanit. thez un certain nombre d'espèces, dans les deux regnes, l'ovule se développe sans fécondation, a l'élat vierge, ou, comme on dit, par parthénogénèsét.

On pourrait prenser que l'élément qui échappe ainsi it la nócessilé de la fécondation, n'est pas un véritable orule; mais il n'en est rien. Il s'agit bien de gametes femel!cs ayant subi toutes les différenciations caractériniques de l'ovogénèse et de la maturation, telles qu'elles ont été exposées plus haut 2 . La parthenocence usl dunc tout à fait distincte des processus de reproduction asexuée que nous étudierons plus loin; cest. au point de rue morphologique, une reproducion sexuce, modifiée par la suppression de ce qui en parait l'acte essentiel, la fusion des gamètes. Elle est définie d'une façon précise par les processus de l'urogénèse. Ils constituent un critérium auquel nous prutons, sans ambages, reconnaitre le gamète femells. Jusqu'à ce qu'on le possédât, la nature précise de l'élément parthénogénétique pouvait,

livre une autorité particulière. La parthénogénèse étant un des aspects principaux de la sexualité, devait, en tout état de cause, avoir sa place dans le présent volume; j'y ai donc maintenu les chapitres écrits, sans aucune modification. Ils constitueront the introduction à l'ouvrage spécial plus approfondi de Delage et Goldsmith, pour le lecteur désireux d'étudier de plus près le sujet, et je souhaite simplement qu'ils éveillent celte curiositẻ.

1. $\pi \alpha \rho \theta$ ق́vos, vierge.

2. Voir Introduction, chap. II et III. 
dans certains cas au moins, rester douteuse. De fait, jusqu'au milieu du $x i x^{\mathrm{e}}$ siècle, on a contesté la réalité du phénomène, au sens propre que nous venons de définir, et, aujourd'hui encore, il reste quelques incertitudes sur l'interprétation de certains modes de déreloppement des animaux inférieurs, pour lesquels on peut hésiter entre la reproduction asexuée et la parthénogénèse, parce qu'on n'a pas pu suivre, avec suffisamment de précision, la genèse des cellules initiales 1 .

La parthénogénèse a été soupçonnẻe, dès l'antiquité, chez certains animaux, par Aristote, nutamment chez les Abeilles. C'est à la fin du xvil ${ }^{e}$ siècle qu'elle a été nettement affirmée chez certains $\mathrm{Pa}_{\mathrm{p}}$ illons. Au milieu du xvile siècle, en 174̇, Bonnet, de Genère, en fit une étude expérimentale chez les Pucerons. Mais jusqu'après les recherches de Siebold, vers 1860, il resta des doutes sur l'équiralence réritable des germes parthénogénétiques et de l'œuf.

\section{§ 2. - La parthénogénèse est un phénomène secon-} daire. - La condition primitive et normale du développement de l'orule est la fécondation; la suppression de celle-ci est l'exception. Mais c'est une exception qui est loin d'ètre rare chez les animaux, et aussi, comme on l'a reconnu au cours des dernières années, chez les régrétaux. Les exemples s'en multiplient graduellement. On peut mème conceroir aujourd'hui que si, dans les conditions naturelles. qui sont suffisantes pour permettre le développement parthénogénétique de certaines espèces, lit parth nogénèse des autres est une impossibilité, par contre, la mise en ceurre d'actions physiques ou chimiques conve-

1. C'est le cas, en particulier, pour la formation des individus, dans certaines phases du cycle exolutif des Trúnatudes formation des Rédies et des Cercaires). 
nablement choisies permettrait, pour les dernières, de la réaliser dans tous les cas. La parthénocrénèse expérimentale est la justification de celte affirmation.

La parthénogénèse naturelle, à l'état rudimentaire, est d'ailleurs extrèmement répandue, même dans les groupes les plus élevés. Tout récemment, Leo Lob signalait, chez des femelles de Cobayes sùrement vierges, des débuts de développiement narthénogénétique fréquents, aux dépens d'ovules restés en place dans l'ovaire. Ces embryons, qui s'arrêtent à un stade plus ou moins précoce, se présentent dans l'oraire, comme de petites tumeurs, ou kystes tératoïdes.

\$3. - Extension de la parthénogénèse. $1^{\circ}$ Animaux. - Passons d'abord en revue les principaux cas actuellement connus de parthénogénèse naturelle et leurs diverses modalités.

C'est l'embranchement des Arthropodes qui en fournit le plus d'exemples. Parmi les Crustacés, chez beaucoup de Phyllopodes (Branchipus, Apus, Artemia), chez la généralité des Cladocères (Daphniss. etc.), chez divers Ostracodes (Cypris), la parthéno⿺rinèse est le mode de reproduction prépondérant. Dans presque tous les ordres d'Insectes, on la trouve, soit chez des espèces particulières, soit dans toute l'étendue de genres, de familles, ou d'ordres ${ }^{1}$.

En dehors des Arthropodes, elle est rraisemhlablement répandue chez les Vers Nématodes, cumme

1. Exemples : de nombreux Phasmides (Orthoptìres), des IIcliothrips (Physopodes), des Trichoptères (Apatania, divers Colénptères (Bromius vitis, Otiorrhynchus turca, et "). limustri parmi les Charançons), des Diptères (Cecidomyia por : 1,..- larves de Miastor); d'assez nombreux Papillons (divers Riming et Sphingides, Psyche helix, des Solenobia). Chez l... Hemiptères, elle est le mode normal de multiplication des I'unetusus et des Coccides. Parmi les Hyménoptères, nous avons vu son rôle 
permettent de le supposer les belles recherches de Maupas. Les Rotifères se multiplient habituellement par parthénogénèse. Dans beaucoup d'autres groupes d'Invertébrés, on en trourerait sans doute des cas plus ou moins fréquents. J'ai eu ainsi l'occasion d'en constater l'existence chez une Annélide Polychète1, groupe où, selon toute vraisemblance, il faut interpréter de mème d'autres exemples de viviparité. Récemment, on a reconnu sa possibilité chez les Dinophilus. Sans entrer dans plus de détails, on roit donc que la parthénogénèse existe naturellement dans des groupes d'animaux très nombreux.

$2^{\circ}$ Végétaux. - Il en est de mème chez les régétaux; mais nos connaissances à cet égard sont beaucoup plus récentes et ne remontent guère qu'à une dizaine d'années. Autant et peut-être plus encore que dans le cas des animaux, le diagnostic précis de la parthénogénèse supposait une étude préalable précise des phénomènes cytologiques de l'orogénèse et de la fécondation, c'est-à-dire des données essentiellement modernes.

Pourtant, dès 1857, Al. Braun avait mis en évidence un cas typique de parthénogénèse chez les Algues de la famille des Characées. Aujourd'hui le développement, sans fécondation préalable, du gamète femelle (oosphère) en une graine a été constaté chez des plantes appartenant à des groupes très variẻs. Je citerai ici diverses Fougères \&, et, parmi les Phanérochez les Abeilles, les Guêpes et les Fourmis; elle est aussi très répandue chez les Tenthrèdes, les Cynipides. Il n'est pas douteux qu'on la décourre encore chez de très nombreuses espèces. en dehors des exemples précédents. Pour plus derdétails, voir Hensegur, les Insectes. Paris, 1904, p. 268 et suiv.

1. Dodecaceria concharum.

2. Athyrium flix femina, Scolopendrium vulgare, Marsilia Drummondi. 
games, l'Antennaria alpina, dioïque, dont les pieds mâles sont trìs rares et où le pollen est atrophié; des Alchrmilla (Rosacées); le Thalictrum purpurascens (Renonculacées); beaucoup de Pissenlits (Taraxacum), des Hieracium, parmi les Composées, etc. Nul doute que l'on en signale de noureaux et nombreux exemples.

Toutefois. il se présente chez les rérrétaux un cas qui leur est propre et qui, tout en réalisant un développement sans fécondation, doit ètre distingué de la parthénorréni:se, sous le nom d'apngamie. C'est celui où la jeune pliante prorient, non d'un gamète femelle, mais de cellules régétatives du parenchyme floral, n'ayant pas la valeur de gamètes. Or. les recherches récentes ont montré qu'il en est parfois ainsi, soit chez les Cryplugames vasculaires (Fougères) ${ }^{1}$, soit chez les l'hanriruganes. Chez une Euphorbiacée australienne (Cielebogyne ilicifolia), où les embryons se développent sans fécondation préalable, Strasburger arait montré, dès 1877 , qu'ils avaient pour origine des cellules de mucelle et non pas des gamètes proprement dits. Il y a lá un ensemble de cas, rentrant dans des catégories assez complexes et que je ne puis analyser ici. Nuus retiendrons seulement que, chez les régétaux, il y a souvent suppléance à la fécondation et dévelopfement d'embryons, soit aux dépens de cellules non germinales des tissus de la fleur (apogamie et processus analogues), soit par une véritable parthénogenèse, de tous points comparable à celle des animaux.

\section{5. - Les divers modes de la parthénogénèse} naturelle. - L'énumération des animaux ou des régétaux ulirant de la parthénogénèse à l'état naturel

\section{Athyrium filix femina, var clarissima, etc.}


n'est pas suffisante pour apprécier l'importance de ce phénomène dans la rie de l'espèce; il faut cncore voir quclle place ii tient dans ce que l'on polil appeler le cycle érolutif des espèces, si la parlirinogénèse coexiste arec la fécondation ou si elle s'est substituée à celle-ci, d'une façon plus ou moins générale et plus ou moins constante. On peut dire que tous les cas existent et c'est ce que montrera une revue rapide des faits.

Parthénogénèse accidentelle ou facultalire. - On désigne ainsi des cas où, normalement. lurule est fécondé, mais où il peut se dérelopper murere quand, par hasard, la fécondation n'a pu se froluire. La parthénogénèse est alors un accident. Des car le cette nature montrent une très grande dirersité. Le puurcentage des cufs non fécondés qui se déveluppent peut ètre très rarié; le développement peut commencer et s'arrèter à un stade plus ou moins arancé. Les phénomènes ont une allure capricieuse. En réalité, ils dépendent de conditions mal comnos, inégalement remplies dans les circonstances naturelles, mais cependant déterminées.

Les Papillons nousoffrent d'assez nombreux cxemples de celte parthénogénèse accidentelle. Dans direrses espèces, la fernelle, bien que soigncusement tenue à l'écart des màles, frond des auf's qui érohnent plus ou moins loiu. Dis le $\mathrm{xwm}^{\mathrm{e}}$ siecle. on a signale te fait chez les Vers à soie. Le déreloppement va raremená jusquàa l'éclosion de la chenille; les tentatises juor l'obtenir ont sourent échoué. Les direrses fares frrésentent des aptitudes très inégales à cet égard. mais il en est, chez lesquelles on a pu ilever completement des individus parlhénngénétiques.

D'antres Bombycirns ou Sphingides ont anssi des œufs qui sont occisionnellement parthénogénéliques. 
On peut citer, à cet égard, le Lymantria dispar dont certains expérimentateurs ont pu obtenir deux et même trois générations consécutives sans fécondation.

Chez les Phasmides, il y à des espèces où les mâles sont très rares (on les appelle pour cette raison oligarrhéniques) et la parthénogénèse y est la règle. Il en est d'autres, où les deux sexes sont également abondants (espèces polyarrhéniques). Or, Pantel, en isolant les femelles de Dixippus morosus, qui appartient à ce second groupe, les a rues pondr es œufs et ceux-ci se sont développés sans féc tion; il a eu ainsi une série de générations unıquement composées de femelles; à la dixième cependant apparurent deux mâles. La fécondation chez les Phasmes parait donc être tout à fait facultative. La parthénogénèse a au moins une très forte tendance à produire des femelles; on dit qu'elle est thélytoque.

La parthén ínèse facultative existe, de même, chez les Hyménuptères du groupe des Tenthrèdes, ainsi qu'il résulte notamment des recherches de Siebold. Si l'on isole des femelles de Nematus ventricosus, espèce dont les mâles sont communs, elles pondent des œufs qui se développent et donnent toujours des mâles; la parthénogénèse chez cet animal, à la différence des Phasmes, est arrhénotoque, comme celle des Abeilles.

La parthénogénèse des Dinophilus rentre dans la même catégorie que les cas précédents; on l'a obtenue, en effet, en se contentant de supprimer, dans les cocons, les œufs mâles et en ne laissant que les cufs femelles. Ainsi les femelles, qui normalement auraient été fécondées, n'ont pu l'être et cependant elles ont pondu des œufs qui ont évolué : les lignées parthénogénétiques sont moins vigoureuses, moins fécondes et n'ont pu être maintenues que pendant un petit nombre de générations. 
A tous les groupes de faits précédents, on pent joindre la parthénogénèse des Hyménoptères sociaux (Abeilles, Guèpes, Fourmis), dont nous arons déjà suffisamment parlé, à propos de la détermination du sexe : la fécondation y est facultative et, suivant qu'elle a lieu ou non, l'œuf devient une femelle ou un mâle. Il semble que cette alternative soit consciente dans une certaine mesare, ce qui donnerait à cette catégorie de faits une valeur particulière; on pourrait la désigner sous le nom de parthénogénèse volontaire. Mais il convient de faire beaucoup de réserves sur cette dernière interprétation.

Parthénogénèse normale. - On désigne ainsi les cas où, a priori, l'œuf ne peut pas être fécondé parce que les mâles n'existent plus. Il arrire même que les femelles deviennent incapables d'être fécondées. La. parthénogénèse est alors obligatoire. Cette condition peut persister pendant un nombre plus ou moins élevé et plus ou moins régulier de générations, après lequel reparaissent les deux sexes et la fécondation. La parthénogénèse est alors cyclique : régulière ou irrégulière. On peut imaginer un très grand nombre de cas rentrant dans cette définition et, en fait, on en connaît une extrême variété. Nous en examinerons rapidement quelques-uns.

Le cycle le plus simple est celui où une génération parthénogénétique alterne régulièrement avec une gẻnération bisexuée dont les œufs sont fécondés. II est réalisé notamment chez les Hyménoptères, dans toute une série d'espèces appartenant à la famille des Cynipides et produisant des galles sur les Chênes. Ce sont de minuscules Insectes, dont la biologie a été éclaircie surtout par les recherches d'Adler. Ainsi que l'a montré cet auteur, il se succède en une année deux générations d'aspects si nettement différents 
qu'elles ont été tout d'abord rangées dans des genres distincts.

Parmi les formes étudiées, nous résumerons ici l'histoire de Iryophanta scutelluris (fir. 31). Pendant l'été, on obsierre souvent, à la face inféricure des feuilles des Chènes de nos régions, des galles sphé-

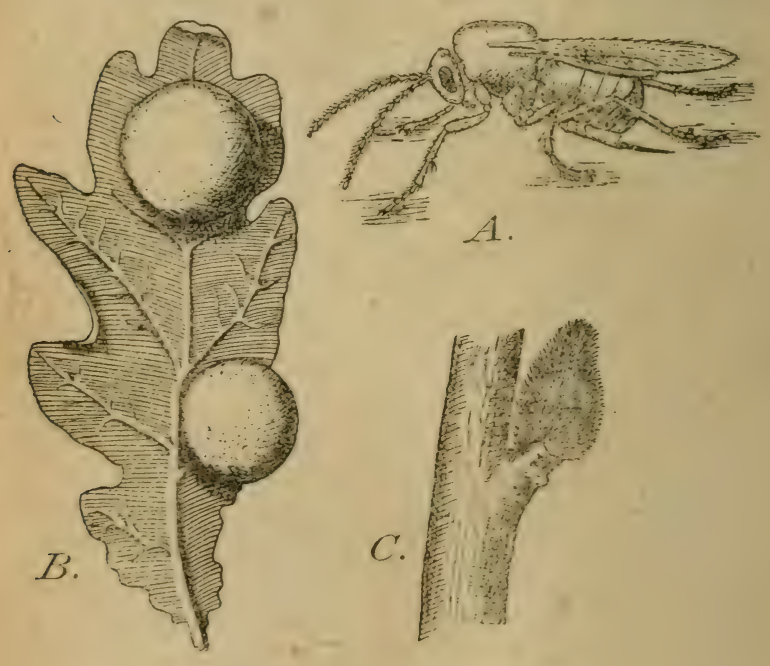

Fig. 31. - Galles de Cynipide.

$A$, un Cyriride (Spathegaster taschenhergi) (dapris Andié:; $B$, Galls autom. nale en crive de Imyophanta scutellaris: I . lialle rintanipre (sur bourgeon de Chine; de Sputheyaster taschenbergi ( $B$ et $C$ ' diapios Adler.)

riques ayant la forme et ie volume d'un rerise. Flles sèchent a l'automne avec la feuille et il on $\rightarrow$ r.t. is la fin de l'hiver, un insecte represuté nuiquentont par le sexe femclle : c'est Dryophlomen sculrlluris. Ces femelles se bitent de pondre - m las bourcirnus des Chenes. encore clos, qu'elles piyment is liarle de leur tarière; elles déterminent ainsi, tu printemps, sur 
ces bourgeons, la formation de pelites salles dont l'aspect et la situation sont, comme on lic vil. lout à fait différentes des précédentes. Les rarlis purius s'y développent et il en sort, vers la lin de mai. des Insectes différents des Dryophanta, auxiqu.is, avant d'aroir suivi leur filiation, on avait donni le nım de Spathegaster taschenbergi; cette géuéralion. wie par parthénogénèse, se compose de mâlesi it de limelles qui s'accouplent. Les femelles fécomlies jmident leurs œufs dans les feuilles du Chène et y déterminent ainsi, dès le début de l'été, les galies cen cerise dont nous sommes partis. Le cycle recomnence; il dure un an et comprend, comme on le roit, deux générations alternant régulièrement ${ }^{1}$.

La parthénogénèse crclique consiste le plus sourent dans la succession d'un certain nombre de générations parthénogénétiques uniquement formées de femelles, après lesquelles s'en produit une, composée de mâles et de femelles dont les œufs sont fécundés. Suivant que ce retour aux conditions habituelles de la reproduction, que nous pouvons appeler gamogénèse, se produit à intervalles réguliers ou irréguliers, nous dirons que la parthénogénèse est cyclique, régulière ou irrégulière. La gamogénèse pent s'espacer de plus en plus, jusqu'à disparaître complète-

1. Ce type de cycle est commun à plusieurs espirces ftudiées par Adler; mais il est susceptible de variations serondaires et d'autre part, il ne s'applique pas i tous les Cynifides. Dans certaines espèces, la génération hivernale n’ẻclûi qui an linut c'e quinze mois et meme de deux ans. D'autres e. pines - telles que Cynips calicis - montrent bien une alternance de générations vivant dans des arbres différents (Quercus jifiluneulata, et $Q$. cerris), mais sans parthénogénèse. Daulres eufir. roinne Rhodites rosa. qui vit sur le rosier, paraissent rire indifiminent parthénogénétiques. La seule famille des Cynipides shez es Hyménoptieres, offre donc une diversité de cas enurtäe $f^{4} \cdot$ ui est loin d'etre entièrement explorée. 
ment; la reproduction devient alors exclusivement parthénogénétique; on dit qu'elle est normale constante.

Parthénngénèse normale constante. - Il est toutefois difficile d'affirmer que ce dernier cas est vraiment réalisé. Il suppose, en effet, l'absence indéfinie des mâles, c'est-à-dire une donnée négative, qu'une constatation positive peut toujours renir infirmer; en fait, il est arrivé assez sourent que des recherches plus approfondies ont mis en éridence des mâles, chez certaines espèces où on ne les connaissait pas encore. Néanmoins, s'il est imprudent d'affirmer sans restriction l'existence de la parthénogénèse rraiment constante, il est certain dans la pratique, pour des espèces de divers groupes, que la gamogénèse y est au moins d'une extrême rareté et d'un caractère tout à fait sporadique.

Les Rotifères, animalcules qui peuplent les mares et les lacs, ne sont généralement représentés que par des femelles parthénogénétiques, ou virginipares, comme nous les avons appelées déjà 1 . Chez beaucoup d'entre eux, les mâles sont encore complètement inconnus, malgré des recherches nombreuses et des cultures prolongées. Il semble donc que beaucoup de ces animaux ne se reproduisent jamais que par parthénogénèse. Il en est de même de beaucoup de cladocères et nous aurons l'occasion de revenir sur leur cas plus loin.

Très significative est l'histoire des Crustacés Phyllopodes et en particulier des Apus. On voit ces animaux pulluler brusquement, surtout dans des eaux recouvrant des terrains inondés. Les œufs peurent subir une dessiccation prolongée et, par suite, sub-

1. V. chap. XIII, p. 165. 
sister dans des vases desséchées, après le retrait des eaux; ils se développent en masse, dès que celles-ci reviennent. Or, pendant longtemps, on n'a connu que le sexe femell de ces animaux. Le mâle a été observé pour la premi re fois, en 185̌6, aux environs de Cracovie; depmi-. il a été retrouvé en dillérentes localités, mais de façon très irrégulière. Ainsi. tandis qu'à Cracuvie. "n 1856, on comptait 1.54 mâles pour 395 femellex. Ans une localité de Franconie, Siebold,

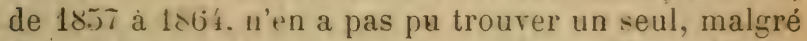
des pèches répétées, comprenant des milliers d'individıs. lieaciun, dautres Phyllopodes (Artemia, Linnadia) se comportent de même.

Il en est encure de mème pour certains Papillons, où la parthénogénèse était déjà soupçonnée par
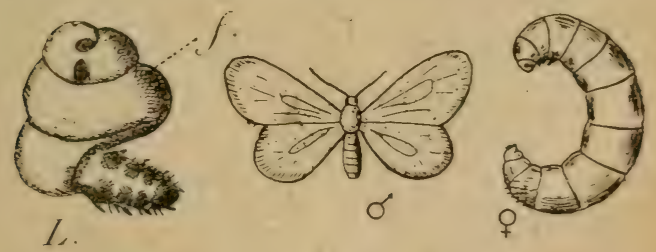

FIG. 32. - Psyche helix.

Le mále $\left(\sigma^{\prime \prime}\right)$ ailé: la femelle (Q) vermitorme; $L$, étui de la larve en formo de limaçon (le corps de la larve fait saillie au dehors). (Empr. a Boas.)

Réaumur au $\mathrm{xv}_{111^{\mathrm{e}}}$ siècle et où, au $\mathrm{xix}^{\mathrm{e}}$, elle a été étudiée arec grand soin par Siebold : Solenobia lichenella et Solpmbia triquetrella, deux Mites, et Psyche helix, un Bombycien. I es chenilles vivent dans des sortes de sacs formés de particules diverses, et ce sac, chez Psyrhe helix (lig. 32), est enroulé comme une coquille d'escargot. Les femelles ne se métamorphosent pas, gardent la forme chenille et pondent dans ces sacs. Le màle de ces Papillons est resté 
inconnu jusqu'en 1860 et n'a été rencontré qu'à l'état de rares exempilaires, et seulement en quelques localités. A la difirence des fernelles, il se métamorphose en Papillun. Chez ces espèces, la reproduction, sauf peut-ètre dans de très rares excertions, est donc exclusivement parthínogérétique. Mais cela leur est spécial et ne sntrinl pas aux espèces les plus roisines. En particulior, latulis que Solenobia triquetrella est indéfininuent farthrungénétique (sous les réserves précédentesi; une :-pice qui peut à peine en être distinguis, q̣ui n'a peut-être que la valeur d'une race, Solenutur pimiti. offre toujours des mâles en abondance et no se reproduit que par œufs fécondés.

Ces uxemples suffisent pour lo moment à montrer dans quulies muditions se présente la parthénogénèe cust-fante. Nous aurons plus loin l'occasion de l'envisager sults d'autres aspects. 


\section{CHAPITRE XVII}

\section{LA PARTHÉNOGÉNĖSE (Suite)}

Déterminisme de la réapparition de la bisexualité, chez les animaux à parthènogénèse cyclique irrégulière.

Cas des Rotifères (Hydatine). - Expériences de Maupas roule de la temperature), de Nussbaum (rôle de la nutrition), de Punnett (rôle de l'hérédité), de Shull et de Whitney (rôle de la composition chimique du milieu).

Cas des Crustacés Cladocères. - Travaux de Weismann : cycles héréditaires et actions du milieu.

Cas des Pucerons : rôle de la température. - Expériences de Kyber, etc.

Le cycle des Chermes. - Travaux de P. Marchal. - Stabilisation progressive des modifications produites par le milieu. - Disparition progressive du sexe mâle (Spanandrie).

Le problème qui se pose est de savoir quel est le déterminisme de ces cycles, quels sont les facteurs qui, à un moment donné, font réapparaitre le sexe mâle et amènent, comme une nécessité plus ou moins impérieuse, la gamogénèse. Il a été très étudié dans ces dernières années, particulièrement sur des Rotifères, des Crustacés Cladocères et des Pucerons; mais on n'a guère réussi qu'à en mettre en évidence l'extrème complexité.

$1^{\circ}$ Parthénogénèse des Rotifères. - Maupas a expérimenté, en 1890, sur un Rotifère, l'Hydatina senta et ses recherches, dont nous avons déjà eu l'occasion 
de parler ${ }^{1}$, ont été le point de départ de nombreux travaux ultérieurs.

Il a montré que, dans les conditions habituelles, les femelles parthénogénétiques de ce Rotifèrene peurent ètre fécondées et produisent de nourelles femelles parhénogénétiques; elles sont virginipares, jusqu'à un vertain moment, où elles donnent naissance à des femelles fécondables; si ces dernières ne sont pas fécondées, elles pondent des œufs petits, qui deviennent des mâles; si, au contraire, elles sont fécondées, elles pondent des œufs à coque épaisse (dits œufs d'hiver ou ceufs durables). Les mères de ces femelles fécondables sont donc, non plus virginipares, mais mères de sexués ou sexupares, et le problème est de savoir les conditions qui font passer de la virginiparité à la sexuparité.

Maupas a cru les trouver dans l'action de la température. En plaçant 5 femelles virginipares à $26^{\circ} \mathrm{G}$., il obtint 106 femelles parthénogénétiques; mais la génération que celles-ci fournirent contenait $97 \%$ de pondeuses de mâles (c'est-à-dire de sexupares) et $3 \%$ seulement de virginipares; tandis qu'une culture parallèle femelle, faite à $15^{\circ} \mathrm{C}$. donnait, à la deuxième génération, $24 \%$ de pondeuses de mâles (sexupares) et $76 \%$ de virginipares. Une température élevée agirait, non pas immédiatement sur la sexualité des œufs de la génération en voie de développement, mais sur la naiure des femelles issues de ces œufs. Une température basse, d'après Maupas, favoriserait la production des virginipares, c'est-àdire tendrait à perpétuer la parthénogénèse; une température élevée arrèterait celle-ci, en déterminant la formation de sexupares.

Ces expériences, reprises par divers auteurs, ont

1. Cf. chap. XIII, p 16 כ 
conduit à des conclusions très contradictoires. M. Nussbaum, en 1897, attribua l'effet constaté par Maupas à la nutrition et non pas à la température; celle-ci n'interviendrait qu'indirectement, en faisant varier la rapidité du déreloppement et, par suite, le nombre des individus. Dans un milieu où la quantité de nourriture reste constante, une population abondante telle qu'en produit une température élevée, se trouve moins bien nourrie; c'était, suivant Nussbaum, cette insuffisance de nutrition qui devait faire cesser la parthénogénèse.

Plus récemment, un auteur anglais, fortement imbu des idẻes néomendéliennes, Punnett, après avoir expérimenté lui-même sur l'Hydatine, a conclu qu'il n'y arait pas d'influence marquée des facteurs externes, tels que la température ou la nutrition, et que la stabilité plus ou moins grande de la parthénogénèse chez les Hydatines dépendait uniquement des propriétés héréditaires des sujets mis en expérience. Il y aurait des lignées possédant une tendance parthénogénétique très forte; d'autres, au contraire, revenant facilement à la gamogénèse. Enfin, deux biologistes américains, Whitney et Shull ce dernier, en faisant aussi une large part à l'hérédité), roient dans la composition chimique du milieu un élément capable d'influer beaucoup sur le retour des Hydatines à la bisexualité.

$2^{\circ}$ Parthénogénèse des Crustacés Cladocères. - Les irustacés Cladocères nous offrent un tableau assez comparable à celui que nous venons d'analyser pour les Hydatines. Il est très aisé d'étudier la reproduction de ces animaux sur les Daphnies, si communes dans les plus petites mares. On ne trouye généralement que des femelles incubant, dans leur carité dorsale, un certain nombre d'embryons en roie de 
développement ${ }^{1}$. A certains moments, se produisent des mâles; d'autre part, l'ovogénèse prend, dans certaines femelles, un caractère très particulier, aboutissant à la formation d'œufs spéciaux fécondables, que l'on appelle, comme chez les Rotifères, œufs d'hiver ou œufs durables. Ils sont plus gros et plus foncés, et chacun d'eux, pour se former, a absorbé un grand nombre d'ovules frères (jusqu'à 47, dans le genre

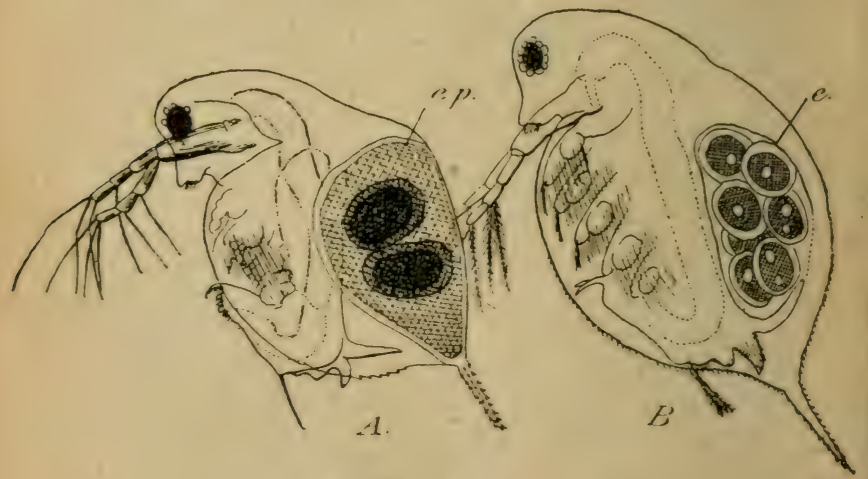

Fic. 33. - Daphnie:

$\boldsymbol{h}$, femelle fécondée montrant dorsalement une éphippie, ep qui renferme deux œufs d'hiver; $B$, une femelle parthénorénétique, avec plusieurs embryons dans sa cavité incubatrice.

Möna); de plus, la paroi de la cavité incubatrice de la mère s'est transformée en une coque spéciale dite éphippie (fig. 33, $A, e p$ ), où l'œuf fécondé se développera lentement, après une période d'arrêt plus ou moins longue. Quel est ici le déterminisme de la production des mâles et de la différenciation des éphippies?

1. Ce développement est rapide. Ainsi, de juin à septembre, on a obtenu, dans des cultures, dix générations successives de Daphnia longispina, en 85 jours. 
Vers 1875, A. Weismann arait fait, à ce sujet, des recherches étendues et remarquables, en étudiant les circonstances naturelles de l'apparition des œufs durables, chez un grand nombre d'espèces et dans des localités très variées. Il était arrivé à la conclusion que chaque espèce présente un cycle déterminé, héréditaire peut-on dire, mais aussi en rapport avec les conditions du milieu où elle vit. Les Cladocères des petites mares ${ }^{1}$, sujettes à des dessiccations fréquentes, produisent plusieurs fois par an des éphippies : elles sont polycycliques. Celles 2 qui habitent des eaux à régime plus constant ne montrent généralement de gamogénèse qu'une fois par an, vers l'automne; il les appelle monocycliques. Enfin, Weismann n'a jamais constaté de mâles, chez certaines espèces vivant dans les grands lacs des Alpes ${ }^{3}$, où le régime est très constant. La parthénogénèse semble y ètre constante et indéfinie. Ces espèces sont acycliques.

La réapparition des mâles et de la fécondation, en amenant la production des œufs durables, capables de résister à la dessiccation ou à d'autres circonstances défavorables, assure donc la persistance de l'espèce en dépit de ces circonstances. Elle est un caractère adaptatif, puisqu'elle se modèle, dans son rythme, sur les vicissitudes du milieu. Mais Weismann ne croit pas devoir admettre qu'il y ait là un effet direct et immédiat des agents extérieurs, au sens lamarckien; il l'interprète, comme tous les faits de même ordre, conformément à ses idées néo-darwiniennes, comme une propriété héréditaire, née indépendamment du milieu et conservée par la sélection.

Sur cette interprétation, des controverses se sont

1. Ex. : Moïna rectirostris, M. paradoxa, Daphnia pulex, etc.

2. Sida cristallina, Daphnia hyalina, Bythotrephes longimanus

3. Bosmina longicornis, B. longispina. 
élevées ultérieurement. Certains expérimentateurs ${ }^{1}$, contrairement à Weismann, attribuent à les facteurs extérieurs, tels que la nutrition, ou la composition chimique du milieu, une action immédiate très efficace pour déterminer, soit la parthénogénèse, soit la gamogénèse : une nutrition abondante, une température élevée entretiendraient la première; la famine, au contraire, déterminerait la seconde. La conception weismannienne des cycles purement héréditaires a, d'autre part, été défendue par des expériences soi-

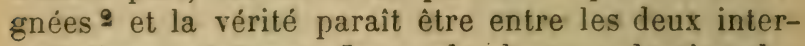
prétations extrêmes. Le mode de reproduction des divers Cladocères, - parthénogénèse ou gamogénèse, - est une réaction aux conditions ambiantes, dont la forme est sous la dépendance des propriètés héréditaires. Celles-ci opposent une inertie plus ou moins considérable à celles-Ià, suivant les espèces et, pour chaque espèce, suivant les moments.

C'est là tout au moins la conclusion des expérimentateurs les plus récents ${ }^{3}$. Chez la Daphnie issue d'un œuf fécondé, la tendance à la parthénogénèse serait tout à fait prépondérante, mais irait en s'affaiblissant, dans les générations parthẻnogénétiques successives qui en sont issues et, pour chacune de celles-ci, dans les pontes successives d'une même femelle. Au bout d'un certain temps, ce serait la tendance à la production de mâles ou d'œufs durables qui deviendrait de plus en plus forte. Entre ces deux phases extrêmes, l'organisme serait dans un état de labilité et d'équilibre instable, sur lequel les actions extérieures ont prise, en faisant pencher la balance d'un côté ou de l'autre. Au contraire, ces mêmes actions ne peuvent pas triompher de la déter-

1. De Kerhervé, Issakovitch, et aussi Langhans.

2. Mme Kuttner, Keilhack, Strohl.

3. Notamment de Papanicolau, Woltereck, Scharfenberg. 
mination très accentuée de l'organisme rers l'une ou l'autre alternative, au début ou à la fin de ces périodes qui constituent les cycles.

Si les faits répondent effectivement à cette théorie, celle-ci préciserait la notion de cycle et en même temps fournirait une explication plausible de résultats qui paraissent contradictoires.

$3^{\circ}$ Parthẻnogẻnèse des Pucerons. - Les Pucerons, ou Aphides ${ }^{1}$, constituent un autre grand groupe d'animaux à parthénogénèse cryclique, pour lequel les expérimentateurs sont aux 'prises avec les mêmes problèmes et sont arrivés à des divergences analogues.

La plupart des espèces passent l'hiver sous forme d'œufs durables pondus à l'automne par des femelles fécondées; il en éclôt au printemps une femelle aptère, la fondatrice, parthénogénétique et vivipare.Par parthénogénèse et viviparité, se succèdent, sur la plante nourricière, au printemps et en été, une série de générations plus ou moins nombreuses, généralement aptères. Elles se nourrissent d'abord aisément sur leur hôte, alors dans la phase la plus active de sa régétation. Au bout d'un certain temps, se produisent des individus ailés, qui émigrent sur d'autres plantes, de la même espèce en général, et y trouvent des conditions de nutrition meilleure; l'apparition des ailés semble en effet corrélative de l'épuisement de la première plante. A la fin de la belle saison. se montre une génération composée de mâles et de femelles qui s'accouplent, et les femelles fécondées, cette fois ovipares, pondent un œuf unique, ou, au plus, quelques œufs, qui subsisteront pendant

1. Ces animaux forment une subdivision importante des asectes Hẻmiptères Homoptères; ils vivent en parasites sur les plantes et sont sourent très nuisibles (ex. : le Phylloxeru de la Vigne): 
Thiver et ne se développeront qu'au printemps. Ic donc, comme chez les Rotifères et les Cladocères, les œufs parthénogénétiques constituent un moyen de développement rapide pendant la belle saison, ol la nutrition est abondante; et la fécondation intervient à un moment où les conditions extérieures deviennent défarorables. L'œuf fécondable a une structure spéciale, permettant la résistance de l'espèce à cette période critique. La parthénogénèse se présente, dans tous ces groupes, comme un mode de multiplication particulièrement rapide, pendant la bonne saison ${ }^{1}$.

L'apparition de la génération bisexuée est-elle déterminée ici par les conditions extérieures - la fin de la régétation estivale et la baisse de la température - ou par des causes internes, qui se sont peu à peu et héréditairement modelées sur le rythme des saisons? C'est le même problème que précédemment.

Déjà, en 1815, Kyber, expérimentant sur le Puceron du Rosier(Aphisrosi), a pu, en le maintenant simplement à température constante, dans des chambres chauffées, faire durer la parthénogénèse, sans intercalation de gamogénèse, pendant quatre années et obtenir ainsi cinquante générations consécutives. Pendant ce temps, des lignées témoins, laissées à la température extérieure, se reproduisaient, chaque automne, par œufs fécondés. Slingerland, qui a répris la même expérience, il y a quelques années, l'a pous-

1. Meme dimorphisme des œufs et meme corrélation avec les circonstances extérieures se rencontrent, en dehors de toute parthénogénèse, chez des Vers plats hermaphrodites du groupe des Turbellariés, dans les eaux douces. Pendant l'été, ils se multiplient par des œufs à déreloppement rapide, s'effectuant dans le corps de la mère; et, à l'automne, ils donnent des ceufs durables, ou œufs d'hiver, à coque épaisse. La signification biologique de ces deux catégories d'œufs, chez ces animaux est tout à fait analogue aux cas que nous étudions ici. 
sée jusqu'à la quatre-ringt-douzième génération sans qu'il se produisit de mâles.

Il semble donc que les facteurs externes - nutrition et température - soient les éléments décisifs de l'apparition de la gamogénèse. Cependant cette conclusion ne serait pas entièrement exacte, selon Th. Morgan, pour certaines espèces, telles que le Phylloxéra du Chêne, tout au moins.

A priori, on doit concevoir, ici comme toujours, que les manifestations de la sexualité sont les résultantes du conflit entre les agents extérieurs et les propriétés héréditaires qui découlent de la constitution même de l'organisme; mais celle-ci, en dernière analyse, n'est-elle pas le résultat de l'action du milieu sur la lignée? C'est ce que mettent admirablement en évidence les recherches récentes sur les Pucerons du groupe des Chermes, en particulier celles de P. Marchal.

Le cycle des Chermes. - Les Chermes sont des Pucerons vivant sur des Conifères, et les travaux d'une série de biologistes ${ }^{1}$ ont établi qu'ils présentent un cycle régulier de générations, avec migrations alternatives sur deux essences différentes.

Voici, par exemple, sommairement résumé (fig. 34), le cycle d'une espèce vivant successivement sur l'Epicéa et le Mélèze : une première génération (1. Fundatrix), issue d'œufs fécondés, vit sur l'Epicéa; elle y produit parthénogénétiquement une seconde génération; celle-ci, à l'état de larves, forme sur l'Epicéa des galles caractéristiques, dites en ananas, puis derient ailée et émigre (II. Migrantes alatæ) sur le Mélèze où elle produit, parthénogénétiquement encore, une troisième génération (III. Exsules) qui

1. Blochmann, Cholodkovsky, Dreyfuss, Bürner, Nüsslin, Marchal, etc. 
hiverne. Au printemps suivant, naît, par parthénogénèse, une qualrième génération (IV. Sexuparæ) ailée qui retourne sur l'Epicéa et y donne naissance, parthénogénétiquement, à une cinquième génération, composée de mâles et de femelles (V. Sexuales). Ceux-ci s'accouplent et les femelles pondent, sur l'Epicéa, des œufs, d'où éclosent les fondatrices, qui recommencent le cycle au printemps suivant. .

Mais ce n'est là qu'une forme typique d'évolution,

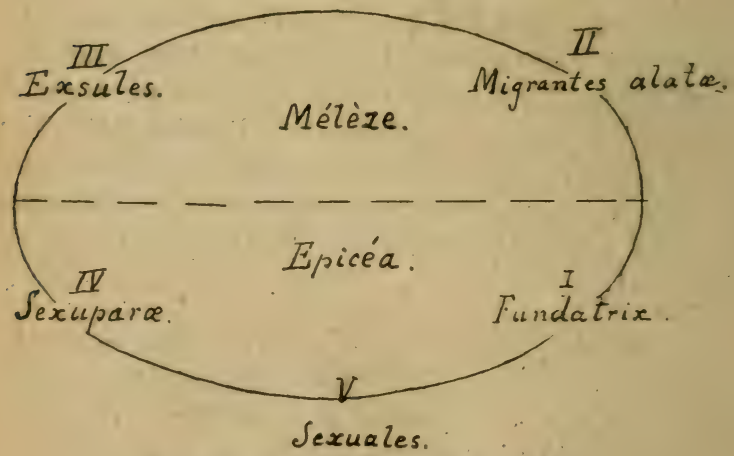

Fig. 34. - Schéma du cycle des Chermes.

- exemple très net de parthénogénèse cyclique régulière, - susceptible de beaucoup de complications ou de modifications secondaires. Elle paraît s'appliquer, par exemple, dans la région du Caucase, au Chermes orientalis, qui vit alternativement sar un Pin et sur le Picea orientalis. Uans d'autres régions de la Russie et en France, or ubserve, sur les Pins sylvestres (Pinus sylvestris), une forme de Chermes (dite Ch. pini) indéfiniment parthénogénétique. Les recherches de Marchal indiquent que ces deux Chermes (Ch. pini et Ch. orientalis) ne sont que deux races physiologi- 
quement distinctes d'une même espèce; entre ces deux races, il n'y a guère de différence morphologique constatable. En l'absence de Picea orientalis, le Chermes s'est adapté à une parthénogénèse indéfinie sur le Pin. De même, le Chermes strobi, qui vit sur le Pin Weymouth (Pinus strobus) et a été importé en Europe avec cet arbre originaire d'Amérique, y est devenu indéfiniment parthénogénétique, parce qu'il ne troure plus, comme dans son pays d'origine, le Picea nigra, sur lequel il effectuait sa reproduction gamogénétique.

Ces espèces, ainsi privées des conditions où elles produisaient leurs sexués, donnent encore naissance aux migrantes alatæ, et même aux sexuparæ, qui végètent sur des essences roisines de celles qui leur conviendraient, mais elles n'y peuvent achever leur cycle par la gamogénèse.

A la longue, ces races, devenues indéfiniment parthénogénétiques, perdent la possibilité de produire la génération sexuée, même si on leur fournit l'arbre convenable. Ainsi, la race de Chermes pini de nos forèts ne donne plus de fondatrices, sur des Picea orientalis plantés à proximité des pins et sur lesquels cependant elle émigre en masse. Marchal, en recherchant la cause de cet avortement de la gamogénèse, a constaté que, parmi les centaines de milliers d'individus pullulant, dans ces conditions, sur des Picea orientalis, il y arait bien production de sexués (sexuales), mais que cette génération ne comprenait que des femelles; il ne se forme plus de mâles ${ }^{1}$. s

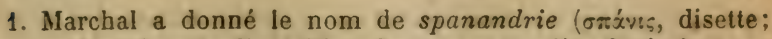
üvríp, mâle), à cette disparition du sexe masculin. Ceci n'es: pas sans analogie avec la disparition des mâles chez les Nénatodes hermaphrodites et l'atrophie de l'instinct sexuel chez les mâles, quand exceptionnellement il s'en forme. (Cf. ch. V, p. 50.) 
Chez le Chermes picex, vivant sur les Sapins (Abies pectinata) de nos campagnes, les sexupares ailés, qui émigreraient, ne se forment plus que très rarement; la reproduction bisexuée est done encore beaucoup plus complètement atrophiée.

On voit, par ces exemples, qu'il se constitue, au point de vue de la reproduction et de la parthénogénèse, des races de Chermes, en harmonie avec les conditions extérieures et chez lesquelles deviennent peu à peu héréditaires des formes de cycles très variées et très différentes de celles qui constituent le type normal du groupe.

Des Pucerons identiques entre eux, au point de rue morphologique, sont ainsi progressivement séparés les uns des autres, par des différences physiologiques ou éthologiques et, de la sorte, à partir d'une souche commune, divergent peu à peu des races, puis des variétés et enfin des espèces indépendantes.

Conclusions. - On conçoit combien est compliquée l'interprétation précise des phénomènes constatés dans la nature; elle ne peut faire de progrès que par une expérimentation rigoureuse. Mais les résultats ainsi obtenus sont des plus intéressants. Ils nous montrent l'origine des adaptations. Au début, il y a modification directe par les agents extérieurs, mais modification dépourvue de stabilité; peu à peu s'établit une transformation héréditaire, de plus en plus indépendante des actions extérieures immédiates.

Les données résumées, dans ce qui précède, sur les Rotifères et sur les Cladocères, relèvent selon toute rraisemblance, des mêmes explications. Là aussi, chaque espèce a dû former peu à peu de nombreuses races physiologiques, adaptées aux conditions extérieures, dans lesquelles elles se sont trouvées pendant une longue série d'années. Ainsi se sont constitués 
les cycles héréditaires plus ou moins stables, que nous constatons aujourd'hui. Leur origine ne peut être conçue que par l'action directe des conditions extérieures, conformément à la doctrine lamarckienne.

Qu'il s'agisse des Rotifères, des Cladocères ou des Pucerons, nous nous trourons, dans tous les cas, en présence des mêmes problèmes. La parthénogénèse y est un processus de multiplication évidemment secondaire, et qui assure une prolifération rapide de l'espèce ${ }^{1}$, dans des circonstances favorables de température, de nutrition, ou plus généralement de milieu. La gamogénèse subsiste aux périodes où, au contraire, les conditions de milieu sont mauvaises; l'œuf fécondé est différencié d'une façon qui permet à l'espèce de surmonter ces moments critiques. Dans les cas où ces derniers ne se présentent plus régulièrement, ou bien si les conditions nécessaires à l'accomplissement de la gamogénèse ne sont plus réalisées (comme nous l'arons vu pour les Cher-

1. Je n'ai indiqué, dans les pages qui prẻcèdent, que les grandes catégories de faits, sans prétendre les passer tous en revue. Dans tous les cas étudiés, c'était l'organisme adulte qui se reproduisait par parthénogénèse. Il en est quelques-uns, chez les Insectes, où la reproduction parthénogénétique a lieu dès l'état de larve, ce qui accentue le caractère accélérateur de ce mode de multiplication. Le cas le plus classique de ce genre est celui de certaines espèces de Diptères, du groupe des Cécidomies, en particulier dans le genre Miastor. Cies larves saprophytes vivent sous l'écorce du bois mort, sur des Champignons, ou dans la pulpe de Betteraves. Elles donnent naissance, par viviparité, à d'autres larves semblables à elles, et il se succède ainsi un nombre de générations plus ou moins élevé, sans qu'intervienne la production d'adultes mâles et femelles. Ces cycles sont, au point de vue biologique, absolument de meme ordre que les précédents et se preteraient sans doute très aisément à une étude expérimentale précise. Cette parthénogénèse se produisant à l'état larvaire a reçu le nom de pædogénèse. 
mes), la parthénogénèse peut se prolonger indéfiniment et il se forme des races, des variétés ou des espèces, qui ont perdu, d'une façon plus ou moins complète, la possibilité de produire des œufs fécondés.

Nulle étude n'est peut-être plus propre à faire apparaître les stades initiaux de la dilférenciation des organismes, l'établissement de divergences physiologriques, qui seront ultérieurement le point de départ de rariations dans la forme et dans la structure. Ces questions o it donc un intérêt très consilérable, non seulement lour le problème de la sexualité, mais aussi pour ct':-: ủe la genèse des espèces. 


\section{đHAPITRE XVIII:}

\section{LA PARTHÉNOGÉNĖSE EXPÉRIMENTALE}

Réalisation expérimentale de la parthénogénèse, par des agents physico-chimiques (J. Løb). - Possibilitẻ générale de l'acti vation de lovule par cette voie. - La dualité de la fécondation - amphimixie héréditaire, activation mécanique de l'orule. - La fécondation est ainsi dépouillée d'un aspect vitaliste.

Extension actuelle et catégories principales des faits de parthénogénèse expérimentale. - Vues sommaires sur les théories proposées pour en expliquer le mécanisme: Cytolyse ef régulation des oxydations (Lœb); coagulations et liquéfactions des colloïdes ovulaires (Delage); déshydratation osmotique et élimination des déchets (Bataillon).

La parthénogénèse naturelle, que nous arons étudiée dans les chapitres précédents, est un phénomène certainement assez répandu dans les deux règnes, mais néanmoins d'un caractère exceptionnel, capricieux et dérivé. La fécondation de l'ovule est la condition primitive universelle.

Aussi la parthénogénèse a-t-elle gardé jusqu'à ces dernières années, dans l'esprit des biologistes, la signification d'une singularité, ou d'une anomalie présentée par un certain nombre de types, et tenant à leur constitution propre, à ce qu'on est courenu d'appeler les propriétés internes de l'organisme. Elle semblait par suite échapper à la prise de l'expérience. Cette conception n'était peut-être pas très logique, mais elle 
était certainement courante et rien ne le prouve mieux que l'étonnement suscité, en 1899, par les résultats annoncés par J. Lœb: la réalisation expérimentale de la parthénogénèse, à l'aide de moyens purement physico-chimiques, chez des organismes où elle n'existe pas à l'état naturel, les Echinodermes. Des zoologistes, parmi les plus qualifiés, tentèrent même d'écarter la signification expérimentale des faits, en les expliquant par une tendance naturelle à la parthénogénèse restée jusque-là inaperçue, chez les œufs des Oursins en question.

Aujourd'hui la parthénogénèse apparaît, au contraire, comme une possibilité générale, pour tous les œufs. On peut concevoir que, moyennant des agents physico-chimiques, dont la mise en œuvre est plus ou moins aisée, tout ovule peut être incité à se développer en un embryon, peut être activé. Le développement, à la vérité, va plus ou moins loin; mais il est, au fond, de même nature que le développement normal, après fécondation. Ce sont des difficultés d'ordre secondaire, qui limitent encore énormément le nombre des exemples de parthénogénèse expérimentale et le degré de développement atteint.

Point n'est besoin d'insister davantage, pour souligner l'extrême intẻrêt de la parthénogénèse expérimentale au point de vue de la biologie générale. J'ajoute que la diversité extrême, déconcertante même, des moyens par lesquels on a réussi à la provoquer, soulève de nombreux problèmes dont la solution est encore très peu avancée. L'étude de la parthénogénèse expérimentale mériterait donc de très longs développements que je n'entreprendrai pas. Je me bornerai à un examen très sommaire des résultats et des explications qu'on en a proposées, ainsi que des conceptions nouvelles qu'ils ont suggérées. Cela me paraît un élément essentiel parmi les problèmes de la 
Sexualité, un complément indispensable aux chapitres qui précèdent.

A J. Lœb appartient d'aroir mis en parfaite évidence la possibilité de la parthénogénèse expérimentale. Mais il avait eu des précurseurs.

En particulier, en 1886, Tichomiroff dans un travail intitulé : La Parthénogénèse artificielle chez les. Insectes, annonçait qu'il avait obtenu la segmentation d'œufs vierges de Ver à soie (Bombyx mori), en les plongeant, pendant une ou deux minutes, dans l'acide sulfurique concentré. Le développement avait été jusqu'à la différenciation de l'ébauche embryonnaire proprement dite; un résultat analogue arait été obtenu en brossant les œufs, à l'aide d'un pinceau.

Ce fait si singulier passa cependant à peu près inaperçu, sans doute parce que le Ver à soie présente. naturellement une tendance plus ou moins accentuée à la parthénogénèse, ainsi que nous l'arons vu, et on fut porté à rapporter à cette tendance les résultats annoncés par Tichorniroff

Dans les années suivantes, plusieurs biologistes, notamment 0 . et $R$. Hertwig, Th. Morgan et quelques autres, faisant agir diverses substances sur les œufs d'Echinodermes, virent également des ovules vierges subir plus ou moins complètement les modifications. cytologiques initiales du déreloppement. Mais ce fut Lœb, qui affirma catégoriquement la possibilité d'un véritable développement parthénogénétique par des actions physico-chimiques, en 1899 et qui le réalisa. Depuis cette époque, entre les mains de Lœb, de ses élèves et d'autres biologistes en Amérique, de Y. Delage et de Bataillon en France, etc., la parthénogénèse expérimentale a été l'un des chapitres les plus féconds et les plus brillants de la biologie contemporaine ${ }^{1}$.

1. On en trouvera un exposé historique détaillè, sous la plume. de Y. Delage, un des auteurs à qui l'on doit les résultats les plus 
La dualitẻ de la fécondation; amphimixie et activation mécanique. - L'une des consérjuences les plus générales de ces découvertes est d'aroir sensiblement modifié l'idée que la généralité des biologistes se faisaient de la fécondation et de son rôle. Ce phénomène arait été envisagé surtout, et souvent exclusivement, sous son aspect morphologique. On avait suivi la destinée du spermatozoïde, après sa pénétration dans l'ovule, par les méthodes de l'histologie. Ce qui derait retenir avant tout l'attention, c'était l'évolution symétrique des noyaux de l'ovule et du spermatozoïde, la résolution de chacun d'eux en un nombre de chromosomes $n$, égal à la moitié du nombre typique des tissus de l'espèce, et par suite la constitution mixte, au point de vue héréditaire, des noyaux de l'embryon.

De là ont découlé toutes les théories cytologiques de l'hérédité, que nous arons rappelées sommairement d'autre part1. La combinaison des hérédités paternelle et maternelle, sous forme de substances chromatiques entrant dans la composition du noyau, l'amphimixie, comme l'a appelée Weismann, a été regrardée comme le phénomène essentiel, et, en vertu des tendances finalistes de notre esprit, comme la raison d'être même de la fécondation ${ }^{1}$.

Toutefois la découverte des centrosomes, vers 1887, et les précisions qui s'y étaient peu à peu ajoutẻes, avaient appelé l'attention sur le rôle dynamique, dans la fécondation, de substances extranucléaires, issues du spermatozoïde. La mécanique de la division de l'œuf fécondé était évidemment dirigée par des forces

importants relativement à cette question, dans les comptes rendus du $\delta^{e}$ Congrès International de Zoologie, à Gratz, en 1910. (Verhandl. VIII,- ntern. Zoologen-Kongresses; Iena G. Fischer, 1912, pp. 100-162.)

1. V. chap. 111, p. 24. 
extérieures à la chromatine. En celle-ci ne pouvait se résumer tout le phénomène de la fécondation. A côté de l'amphimixie, image, tout au moins, de la combinaison des hérédités paternelle et maternelle, il y avait une action mécanique, dynamique, exercée par le centrosome et celui-ci semblait provenir du seul spermatozoïde. Le spermatozoïde agissait donc de deux façons: par la chromatine de son noyau, pour réaliser l'amphimixie et par son centrosome, pour provoquer mécaniquement la division de l'œuf, inerte sans cela.

L'activation naturelle ou expérimentale de l'ovule est un phénomène d'ordre physico-chimique. - Or, la parthénogénèse expérimentale nous montre, qu'un œuf, qui, dans les conditions naturelles, aurait dù être fécondé pour se développer, évolue sans cette double action du spermatozoïde, moyennants un traitement chimique, qui ne supplée nullement à l'amphimixie, mais réalise l'équivalent mécanique de l'action stimulante du centrosome. Cela montre que l'amphimixie n'est pas une condition indispensable au déreloppement de l'œuf. Sans doute, elle joue, dans la nature, un rôle capital, au point de rue physiologique, dans la conservation des propriétés de l'espèce; mais cependant tout orule peut se développer en un embryon sans avoir reçu de chromatine d'un spermatozoïde. On peut donc logiquement concevoir que l'action dynamique du spermatozoïde est l'élément le plus strietement indispensable et, par suite, essentiel dans la fécondation. Celle-ci apparait alors, avant tout, comme l'activation mécanique de l'ovule.

Il ne faut cependant nullement, je le répète, rabaisser l'importance effective de l'amphimixie. Elle domine tout le problème de l'hérédité, qui est bien la propriété la plus essentielle des organismes. 
En se plaçant au point de vue le plus général dans l'étude de la Biologie, la parthénogénèse expérimentale est, à un autre égard, un progrès de premier ordre. La fécondation perd, en effet, par elle, un de ses aspects vitalistes. Elle devient, au point de vue dynamique, un phénomène purement physicochimique. Le spermatozoïde de l'espèce n'est plus, par une sorte de finalité fatale, l'instrument nécessaire du développement de l'ovule. Des agents purement physico-chimiques peuvent en tenir lieu.

Cette notion résultait d'ailleurs en partie des faits relatifs à l'hybridation et surtout à l'hybridation entre espèces éloignées, faits que nous devons aussi pour une large part à Lœb.

Jusqu'à lui, on ne connaissait guère l'hybridation qu'entre races ou espèces très voisines, dont les gamètes, ovules ou spermatozoïdes pouvaient être considérés comme quasi-identiques. Il n'y avait rien de très surprenant à ce qu'ils se suppléent réciproquement d'une forme à l'autre. Cela ne heurtait pas l'idée que l'ovule exigeât, pour se dérelopper, le spermatozoïde même de l'espèce, comme par une sorte d'harmonie préétablie.

Or, Lœb a réussi, en 1903, à féconder un Oursin par du sperme d'Etoile de mer, et cela, moyennant une simple modification dans le degré d'alcalinité de l'eau de mer. Godlewski, de même, a pu féconder l'ovule d'un Oursin, par le spermatozoïde de Comatule. Dans les deux cas, les spermatozoïdes appartenaient à une classe différente du même embranchement, les Echinodermes. Lœb et Kupelwieser ont même fécondé l'œuf d'Oursin, par du sperme de Mollusque, c'est-à-dire d'un animal d'embranchement différent. En présence de ces résultats, il n'y a plus aucune finalité plausible dans la structure du spermatozoïde correspondant à l'ovule. Ajoutons que, dans 
les deux premiers de ces cas, il y a une fécondation totale véritable, avec ses deux éléments, amphimixie et actiration mécanique; dans le troisième (Oursin Mollusque), le phénomène amphimixique ne se produit plus, les deux noyaux, de compositions trop éloignées, ne se fusionnent plus et l'action du spermatozoïde se réduit à l'activation mécanique.

Extension actuelle des faits de parthénogénèse expérimentale. - La parthénogénèse expérimentale, c'est-à-dire l'activation de l'ovule vierge par des moyens physico-chimiques, a été obtenue actuellement sur des animaux extrêmement variés : de nombreux Echinodermes (Oursins, Etoiles de mer, Holothuries), des Annélides (Nereis, Chétoptère), des Géphyriens (Thalassema, Phascolosoma), des Mollusques (Lottia, Acmæa, Mactra), des Némertiens (Cerebratulus) entre autres Invertébrés; des Poissons et des Batraciens, chez les Vertébrés. Le nombre des. types a été limité beaucoup plus par les difficultés matérielles de l'expérience, que par la nature des animaux.

Les stades jusqu'où ont érolué les œufs activés sont très variables. Dans certains cas, on n'a pas dépassé les premières phases de la segmentation. Mais avec les Echinodermes, on a obtenu, par plusieurs méthodes, des larves (Pluteus, Bipinnaria) et même Delage a réussi à pousser celles-ci au delà de la métamorphose et à obtenir quelques jeunes Oursins. C'est là une réussite extrêmement difticile, même dans les conditions naturelles de la fécondation : l'élevage des jeunes larves, à partir du moment où elles doivent étre nourries, est, en effet, pratiquement, des plus malaisés. Bataillon, de son côté, après n'avoir obtenu, pendant une série d'années, sur des Poissons et des Amphibiens, que les phénomènes initiaux du dére- 
loppement, a conduit des Grenouilles et des Crapauds parthénogénétiques, non seulement jusqu'au têtard, mais au delà de la métamorphose de celui-ci, jusqu'au jeune animal terrestre, et divers expérimentateurs ont confirmé ses résultats.

Le développement que l'on obtient par la parthénogérèse expérimentale n'est donc pas un processus pathologique et limité; mais il est tout à fait comparable au développement normal et peut, en particulier, être identifié à la parthénogénèse naturelle. Celle-ci a dù résulter simplement de la réalisation, à un moment donné, de conditions physico-chimiques convenables, qui ont rendu superflue l'activation par le spermatozoïde, et qui, dans certains cas, se sont régulièrement répétées ou même sont devenues constantes ${ }^{1}$.

Théories sur le mécanisme de la parthénogénèse expérimentale. - Il va de soi que l'intérêt scientifique véritable de la parthénogénèse expérimentale est dans l'analyse des conditions qui la provoquent, dans la connaissance de son déterminisme. Malgré douze années de recherches multiples, ce problème est encore peu avancé. Les agents, par lesquels on a provoqué la parthénogẻnèse, sont très variẻs; il est difficile d'aperceroir les éléments de leur action qui leur sont communs. On a, en effet, actiré les orules, en les plongeant, pendant un temps convenable, dans des solutions salines hypertoniques ${ }^{2}$, puis dans des solutions sucrées, dans des solutions d'acides minéraux

1. Si l'expérimentateur n'obtient parfois qu'une ébauche de développement partlénogénétique, il en est de méme souvent dans la nature. On connait, en effet, aujourd'hui, chez d'assez nombreuses espèces, une parthénogénèse naturelle rudinuentaire.

2. C'est-à-dire plus concentrẻes en sels que les liquides dans lesguels on trouve normalement rovule. 
ou d'alcalis, dans le sérum sanguin d'autres espèces; en leur faisant subir de simples actions mécaniques (télles que le secouage dans un tube); en les exposant à des températures élevées $\left(32^{\circ}, 33^{\circ}\right)$ ou basses $\left(+4^{\circ}\right.$ à $+7^{\circ}$ ), ou enfin en leur faisant subir un traumatisme léger. Bataillon a, en effet réalisé la parthénogénèse des Grenuuilles et des Crapauds en piquant simplement l'ovule mùr arec une très fine aiguille.

Ces agents sont, comme on le roit, extrèmement hétérogènes : je me bornerai ici à mentionner les conceptions les plus générales parlesquelles on a tenté d'expliquer, - en l'état encore très peu arancé de nos connaissances sur la physico-chimie des protoplasmes, - le mécanisme de leur efficacité.

Théorie de J. Lœb. - Lœb, qui a multiplié les expériences sur les Echinodermes, est arrivé à la conception suivante. Dans la fécondation, dès que le spermatozoïde a pénétré dans l'ovule, il sa forme, à la périphérie de celui-ci une zone hyaline limitée par une membrane transparente, qui sert de barrière aux autres spermatozoïdes. Lœb considère ce phénomène comme une liquéfaction, une cytolyse des couches superficielles de l'ovule, sous l'influence d'une substance introduite arec le spermatozoïde, une lysine; cette cytolyse s'arrète bientôt. Dans les premiers procédés de parthénogénèse expérimentale, la membrane ne se formait pas; le développement de l'œuf était d'une aliure très différente de ce qu'il est normalement.

Mais Lœb a réussi ensuite à imiter complètement le développement normal. Il y parvient en traitant les ovules par des solutions d'acides gras ${ }^{1}$ (acides for-

1. On les plonge, pendant quelques minutes, dans $50 \mathrm{oc}$. d'eau de mer, additionnée de 2 cc. d'une solution décinormale d'acide butyrique, par exemple (la solution normale d'un sel est 
mique, acétique, butyrique valérianique, etc.) Il se forme alors une membrane autour de l'ovule, tout comme par la fécondation, et la segmentation a tout d'abord l'allure naturelle. Toutefois la cytolyse, d'où résulte la formation de la membrane, et qui, dans le cas de la fécondation, s'arrète bientôt, se poursuitici, en général et bientôt elle atteint tout l'œuf, qui meurt. L $\propto b$ a su enrayer cette cytolyse fatale à l'embryon, par un second traitement appliqué aux œufs après celui par les acides gras. Il les plonge dans une solution hypertonique ${ }^{1}$. Moyennant ce procédé, les œufs se développent exactement comme s'ils avaient été fécondés.

C'est ce que Lœb explique a une raçon très ingénieuse, mais un peu subtile. Il résulte d'expériences faites par lui que l'activation de l'ovule s'accompagne d'une augmentation considérable des oxydations, dont le cytoplasme est le siège. Dans la fécondation, il s'établit un rythme convenable, entre la rapidité des processus d'oxydation et la cytolyse, tandis que, par l'action des acides gras, le rythme de ces phénomènes est mal réglé. L'øuf est malade et succombe.

On le guérit en suspendant temporairement les oxydations; par exemple, en plongeant les œufs, pendant un temps convenable, dańs une solution très faible de cyąnure de potassium, qui inhibe les oxydations. Le traitement par les solutions hypertoniques a un résultat du même ordre; son effet doit être de régler celle qui contient le poids moléculaire en grammes de ce sel dans un litre d'eau; la solution décinormale est la précédente, diluée dix foris).

1. Après l'action de la solution d'acide gras, les œufs sont remis dans l'eau de mer ordinaire, pendant 15 à 20 minutes, puis placés pendant 15 à 60 minutes dans un mélange de $30 \mathrm{ce}$. d'eau de mer et de $8 \mathrm{cc}$. d'une solution de sel marin renfermant deux fois et demie le poirs moléculaire en grammes par litre. 
les oxydations à une allure compatible avec la vie des cellules ${ }^{1}$.

Delage et Bataillon ont formulé diverses objections importantes à cette interprétation et proposé d'autres théories dont j'indiquerai rapidement les points essentiels.

Théorie de Delage. - L'ovule est, ainsi que le remarque Delage, un complexe de substances colloïdes instables. Son développement peut être envisagé comme une série de coagulations et de liquéfactions alternatives de ces colloïdes, passant par des états de gel et de sol. La formation de la membrane autour de l'ovule serait une coagulation, la disparition de la membrane nucléaire serait une liquéfaction. L'ovule, par lui-même, serait incapable de commencer spontanément cette série de transformations; mais une fois celle-ci amorcée, elle se continuerait d'ellemême, à la façon de nombreuses réactions chimiques. Dans cette mise en branle consisterait le rôle dynamique de la fécondation et aussi celui des agents de la parthénogénèse expérimentale. Delage (qui conteste la nécessité des circonstances déclarées essentielles par Lœb et notamment le rôle des oxydations) voit une vérification de de ses propres idées dans le fait qu'il a. réalisé un procédé de choix pour la parthénogénèse des Echinodermes, en soumettant les ovules à l'action successive (ou même simultanée) de solutions coagulantes et décoagulantes 2 .

Tout indique actuellement que les phénomènes de l'activité cellulaire sont en relation étroite avec la structure colloïdale du cytoplasme; mais nous

1. Pour le dẻveloppement de ces idées, voir : J. LofB, la Fécondation chimique (trad. A. Drzerwina, au Mercure de France, 1911).

2. Delage fait agir successivement des alcalis et des acides 
sommes encore peu arancés dans l'étude de cette chimie d'une extrème complexité, et, en reconnaissant tout le mérite et la sagacité des solutions orientées vers clle, nous ne devons pas nous dissimuler que des apparences telles que la formation ou la dissolution des membranes ne sont peut-être que des conséquences très indirectes des actions vraiment lécisives et immédiates dans l'activation de l'ovule. Cela ne diminue en rien la valeur des méthodes par lesquelles Delagre a obtenu, chez les Echinodermes, des déreloppements parthénogénétiques, avec une perfection égalant le déreloppement normal; et il est jusqu'ici le seul expérimentateur qui, malgré les difficultés toutes particulières de l'élevage de ces animaux, au delà de leur. métamorphose, ait réussi à pousser des embryons parthénogénétiques jusqu’à un état comparable à l'adulte.

Théorie de Bataillon. - Bataillon, dès 1900, s'était attaqué aux Vertébrés, c'est-à-dire à l'embranchement le plus élevé de la série animale, pour y provoquer la parthénogénèse. Après n'avoir obtenu pendant dix ans, par les procédés les plus variés, que des débuts de développement plus ou moins rudimentaires, il a réussi récemment, par une simple piqûre de l'ovule, chez la Grenouille et chez le Crapaud, à le faire évoluer comme après fécondation ${ }^{1}$.

ou bien des solutions de tanin (coagulant) et d'ammoniaque (liquéfiant) :
A $\{$ Eau de mer. ...... $50 \mathrm{cc}$.
A Solution décinormale de tanin. . 28 gouttes pendant 5 minutes.
$\boldsymbol{B}\left\{\begin{array}{l}\text { Eau de mer. . . } 30 \mathrm{cc} \text {. } 70 \mathrm{cc} \text {. pendant quelyues minutes. } \\ \text { Solution iso'onique de saccharose. } 30 \text { gouttes. }\end{array}\right.$

1. En réalité, ce n'est pas la piqûre pure et simple qui provoque le développement. En la faisant, Bataillon introduisait Jans l'ovule un peux de sang, et il a montré que cette circons- 
Depuis l'origine de ses recherches, il a émis l'idée que le mécanisme essentiel de la parthénogénèse expérimentale résidait d'une part dans des phénomènes d'osmose déshydratant l'orule, de l'autre, dans l'élimination par l'œuf de matériaux de déchet. Cette seconde hypothèse reste, malgré tout, bien vague. Quant à la première, tout le monde sait l'énorme importance des phénomènes d'osmose dans le fonctionnement des cellules et l'efficacité des solutions hypertoniques comme agents de parthénogénèse expérimentale plaiderait fortement en sa faveur, si d'autres agents tout aussi efficaces ne paraissaient mettre hors de cause les phénomènes osmotiques. L'expérience si brillante de la parthénogénèse par piqûre ne s'explique que bien difficilement par ces mécanismes.

Conclusion. - En somme, comme le reconnait Delage, la théorie véritable de la parthénogénèse expérimentale est à faire et les quelques pages précédentes n'ont nullement la prétention de discuter à fond le problème ${ }^{1}$.

Si nous sommes encore peu arancés dans l'interprétation du phénomène, nous ne devons pas perdre de vue son importance et sa généralité, en tant que fait. Sur des organismes les plus variés, on a pu activer l'œuf, par des moyens multiples et purement physico-chimiques. Nous pouvons ainsi entrevoir comment, par le jeu d'éléments analogues relevant du milieu extérieur, s'est réalisée la parthénogénèse naturelle, sous les diverses formes où nous l'arons constatée.

tance avait une importance capitale. Le sang de grenouille peut d'ailleurs etre remplacé par du sang d'autres espèces animales. Je ne puis ici entrer dans l'exposé détaillé de cette question.

1. Voir pour une discussion plus complèté, le livre de Delage et M. Goldsmith : la Parthénoyénèse naturelle et expérimentale. 
D'une manière générale, qu'il s'agisse de parthénogénèse naturelle ou de parthénogénèse expérimentale, l'évolution de l'ovule en un embryon est dépouillée du caractère mystérieux et vitaliste que Iui conféraient les anciennes conceptions sur la fécondation. A bien réfléchir, le développement de l'œuf en un embryon n'est qu'une division cellulaire suivie d'une série d'autres et ne doil pas relever de mécanismes fondamentalement différents de n'importe laquelle des divisions ultérieures. Mais si la logique pure nous permet de penser ainsi, les constatations de faits qui sont la vérification de cette idée n'en ont pas moins une importance et une fécondité considérables 


\section{CHAPITRE XIX}

\section{LA PARTHÉNOGÉNĖSE ET LA RÉDUCTION CHROMATIQUE}

Comment la réduction chromatique dans l'ovule est-elle compensée, en l'absence d'une fécondation? - Le nombre des globules polaires dans les œufs parthénogénétiques. - La réduction chromatique chez le mâle des Abeilles. - Cas de conjugaison du second globule polaire et du noyau de l'ovule. - Possibilité d'une autorégulation du nombre des chromosomes.

On a ru ${ }^{1}$ quelle importance a été attribuée par les biologistes contemporains, dans les problèmes de l'hérédité et du sexe, aux chromosomes, c'est-à-dire aux anses chromatiques en lesquelles se résout le noyau, lors de la division cellulaire. Le nombre de ces anses est réduit de moitié, de $2 n$ à $n$, lors des deux divisions maturatives, c'est-à-dire immédiatement avant la fécondation, et la fécondation - fusion de l'ovule et du spermatozoïde, dont chacun apporte dans son noyau $n$ chromosomes - assure le rétablissement du nombre $2 n$ dans l'œuf et les tissus de l'embryon. Qu'advient-il dans le cas de l'ovule parthénogénétique? L'apport du spermatozoïde manquant, comment le nombre des chromosomes est-il maintenu constant d'une génération à l'autre? Question

1. Ch. III, p. 24 ; ch. XIV, p. 178. 
capitale pour ceux - et ils sont les plus nombreux qui roient dans les chromosomes le substratum de l'hérédité et qui leur assignent permanence et individualité. On peut imaginer, a priori, l'existence d'un mécanisme régulateur spécial dans les œufs à parthénogénèse normale et obligatoire; mais que se passet-il dans ceux où la parthénogénèse est accidentelle ou provoquée expérimentalement?

Nombre des globules polaires dans les œufs parthénogénétiques. - La maturation des œufs parthénogénétiques a commencé à être étudiée, vers 1885 , avant que les théories sur les chromosomes et sur la réduction chromatique eussent pris leur forme actuelle. Un fait sembla se dégager nettement alors : les ceufs parthénogénétiques n'émetraient qu'un seul globule polaire au lieu de deux. Il en est bien ainsi dans la plupart des cas, mais il y a des exceptions. On décourrit, en effet, deux globules polaires dans les œufs parthénogénétiques de l'Abeille, de diverses Foúrmis (Lasius), de Papillons (Lymantria dispar), les femelles pondeuses de mâles chez les Rotifères (Asplanchna priodonta). Toutefois, on peut remarquer zue, dans tous ces cas, il s'agit de parthénogénèse accidentelle ou facultative. Il n'est pas dans la destinée de ces orules de n'être pas fécondés. Rien d'étonnant, dès lors, à ce que les phénomènes de la maturation s'y accomplissent normalement. Mais on a retrouvé encore les deux globules polaires chez des œufs à parthénogénèse obligatoire, par exemple chez ceux d'un Cynipide à parthénogènèse normale indéfinie, Rhodites rosæ. Il y a donc là une exception indéniable à la règle précẻdente.

Considérons seulement, pour le moment, les œufs parthénogénétiques qui n'émettent qu'un globule polaire: ils restent e cas général. On a pu croire 
que la suppression de la seconde division maturative évitait la réduction chromatique, lorsqu'on admettait que cette réduction n'était effective qu'à cette seconde division. Mais il est maintenant à peu près universellement admis que la réduction, tout au moins numérique, des chromosomes est effectuée, arant mème la formation du premier globule polaire ${ }^{1}$ et la réduction qualitative (pour ceux qui admettent l'individualité des chromosomes) doit s'effectuer à la première division maturative, c'est-à-dire lors de la formation du premier globule polaire. Ainsi, étant donnée la conception que se font aujourd'hui des chromosomes et de la réduction chromatique, la majorité des biologistes, les œufs parthénogénétiques subissent cette réduction, qu'ils expulsent un ou deux globules polaires.

Existe-t-il alors en eux des mécanismes compensateurs, et, de toute façon, qu'arrive-t-il pour les œufs à parthénogénèse, facultative ou expérimentale qui ont subi leur maturation, à la façon ordinaire? La réponse à cette question est loin d'ètre actuellement claire et complète.

Cas de l'Abeille mâle. - Il est très intéressant de l'envisager d'abord chez les mâles d'Abeilles, issus, comme nous avons dit, d'œufs facultativement parthénogénétiques 2. L'histoire en est curieuse, même par les erreurs qui ont été commises; celles-ci montrent la puissance d'idées préconçues dans des problèmes de ce genre et doivent mettre en garde contre beaucoup d'affirmations trop catégoriques, eu égard aux moyens d'investigation dont on peut disposer. Sous l'influence évidente des idéas de

1. A la phase de synapsis.

2. V. ch. XIII, p. $16 \%$. 
Weismann, relatives à la signification des chromosomes et à la réduction chromatique, Petrunkevitch étudia, en 1901, la maturation et le développement de l'œuf parthénogénétique d'Abeille. Il y confirma la formation de deux globules polaires, qui restent inclus dans le cytoplasme de l'œuf. Mais alors qu'on roit, dans tout le règne animal, les globules polaires dégénérer sans prendre part à la formation de l'embryon, Petrunkevitch admit qu'ils étaient incorporés à celui-ci, s'y divisaient et y formaient les testicules. D'autre part, il décrivait la fusion préalable des deux globules polaires en un seul noyau renfermant $2 n$ chromosomes. Ainsi, bien qu'il n'y eût pas eu fécondation et que, par suite, les tissus des mâles dussent renfermer seulement $n$ chromosomes au lieu de $2 n$, ce dernier nombre se trouvait rétabli dans les cellules germinales, souche de la génération suivante.

Il n'y aurait qu'à s'incliner devant ces faits, s'ils étaient effectivement établis. Mais Petrunkevich n'a apporté et ne pouvait apporter, par sa méthode d'observation, aucune preuve véritable que les globules polaires donnent bien naissance aux glandes génitales; et c'est là un paradoxe exigeant une preure catégorique. Or, en 1904, un observateur excellent, F. Meves, étudiant la spermatogénèse des Abeilles, y constata une particularité tout à fait inattendue, qui semble assurer la constance du nombre des chromosomes, sans exiger d'admettre un processus aussi étrange que la genèse des testicules aux dépens des globules polaires. Meves a constaté, en effet, que la première des deux divisions maturatives, celle des auxocytes en préspermatides ${ }^{1}$, avorte. La division du noyau s'ébauche, mais s'arrête et l'un€ des deux préspermatides n'est représentée que

1. Cf. ch. III, p. 27. 
par une petite masse cytoplasmique non nucléée. La seconde division a lieu et donne deux spermatides, dont l'une seulement est bien développée ef devient un spermatozoïde. Grâce à l'avortement de la première des deux divisions maturatives, il n'y a pas de réduction chromatique à la fin de la spermatogénèse, et si les divers tissus du mâle d'Abeille n'ont, par suite des circonstances de la parthénogénèse, qu'un nombre $n$ de chromosomes (au lieu de $2 n)$, - ce qui n'a pas pas encore pu être effectivement vérifié - ce nombre n'est pas réduit à noureau dans les spermatozoïdes et se trouve le même que dans le cas général. Il y a donc là un mécanisme régulateur particulier qu'on n'eût pas imaginé $a$ priori; son exactitude se troure d'autant mieux assurée par cette dernière circonstance et aussi par les confirmations qu'en ont données, après Meves, des auteurs très qualifiés ${ }^{1}$, chez des genres voisins d'Hyménoptères.

On a trouvé, d'une maniere plus ou moins nette, dans l'étude d'autres œufs parthénogénétiques, des mécanismes équivalents. L'un d'entre eux consiste en ce que, dans des œufs parthénogénétiques qui n'expulsent qu'un seul globule polaire, le second est ébauché, mais non expulsé, et que son noỵau se fusionne avec le noyau définitif de l'ovule, jouant ainsi le rôle ordinairement dévolu au spermatozoïde. Brauer a décrit ce processus comme réalisé, dans une partie tout au moins des cufs parthénogénétiques d'un Crustacé Phyllopode, l'Artemia salina. Divers observateurs signalent la même fusion, entre le noyau du second globule polaire et celui de l'ovule, dans le

1. Mark et Copeland, Meves et Duesberg, chez les Guepes; Lams et Schleip, indépendamment l'un de l'autre, chez les Fourmis: Doncaster dans la génération parthénogénétique d'un Cynipide du Ghene, Neuroterus lenticularis. 
cas de la parthénogénèse expérimentale, chez l'Etoile de mer. Mais les faits eux-mêmes mériteraient une confirmation indéniable.

$Y$ a-t-il une autorégulation du nombre des chromosomes? - Il y a bien une hypothèse beaucoup plus simple, mais peu en faveur actuellement, parce qu'elle est contraire à la notion généralément admise de l'individualité et de la permanence des chromosomes : c'est que, si, dans l'ovule parthénogẻnétique et les premières cellules de l'embryon, le nombre des chromosomes est réduit à $n$, peu à peu, par une autorégulation, ce nombre redevient $2 n$. Cela suppose, éridemment, que les chromosomes ne sont pas des individualités absolues, mais que, par un processus ou un autre, il puisse s'en former de nouveaux. Or, en admettant même la légitimité des conclusions tirées des données actuelles immédiates de la cytologie, rien ne prouve l'impossibilité de cette dernière supposition. Aucun fait positif, il ne faut pas l'oublier, ne prouve formellement l'individualité et la permanence des chromosomes; les raisons indirectes les plus favorables à cette conception ne vaudraient que pour les premières phases du développement, pour les premières divisions de l'œuf. Il est abusif de les étendre davantage. Comme l'a fait remarquer Meves; il serait possible que les édifices chromosomiques se conserrent plus ou moins, d'une division à l'autre, au début de la segmentation, où ces divisions se succèdent très vite; mais il n'en serait pas forcément de même pour les divisions ultérieures, séparées par des périodes beaucoup plus longues. A ce moment, il pourrait se constituer de nouveaux chromosomes et jamais on n'a pu faire une suite continue d'observations sur l'ensemble du déreloppement à ce point de vue. D'autre part, Delage avait cru observer, chez les 
Oursins, dans un processus analogue à la parthénegénèse, - la mérogonie ${ }^{1}$, - que le nombre de chromosomes réduit au début à $n$, remonte peu à peu à $2 n$, par une autorégulation. Le fait a été contesté par divers observateurs ultérieurs; mais, ell admettant mème l'exactitude de leurs constatations, leur négation ne s'applique qu'aux premiers stades du développement, où il semble bien effectivement que le nombre reste réduit à $n$. Il faudrait des observations précises sur des stades avancés et si elles ne sont pas encore faites, c'est qu'elles sont beaucoup plus difficiles à cause de la petitesse des éléments. Mais, en leur absence, on ne peut nier formellement l'autorégulation.

D'autre part, en étudiant, au point de vue cytologique, des tètards parthénogénétiques de Grenouille obtenus par le procédé de Bataillon (piqûre de l'œuf vierge), Brachet a trouvé, chez ces embryons, des noyaux en division caryocinétique, offrant des nombres de chromosomes variés et supérieurs au nombre réduit ( $n=12$ chez la Grenouille). Ses obserrations lui ont paru insuffisantes pour formuler une conclusion précise, mais on peut espérer qu'elles pourront ètre complétées à brève échéance. La parthénogénèse expérimentale parait, en tout cas, chez les Amphibiens, un terrain de choix², pour la solution

1. C'est le développement en un embryon d'un fragment d'ovule dépourvu de toute trace du noyau ovvulaire, mais fécondé par un spermatozoïde. Giard a fait justement remarquer que le processus équiralait au développement parthénogẻnétique du spermatozoïde, impossible dans les conditions normales, parce que le spermatozoïde est dépourvu de toutes réserves nutritives. Celles-ci lui seraient précisément fournies par le fragment d'ovule où il a pénétré.

2. Ce n'est pas ici le lieu de traiter ce problème à fond, mais j'indiquerai cependant qu'en étudiant la parthénogénèse rudimentaire des Ciseaur, dirers observateurs et entre autre 
par les faits d'une controverse à laquelle est attachée actuellement une importance considérable.

L'aurait-elle, si on se résignait à ne pas prêter gratuitement aux chromosomes la signification qu'on leur attribue au point de vue de l'hérédité? La conception qu'on s'en fait aujourd'hui est fondamentalement entachée d'un vitalisme, que rien ne justifie au fond. Peut-être des considérations physico-chimiques, déduites des propriétés de la structure colloïdale du cytoplasme et du noyau, suffiraient-elles à rendre compte des apparences diverses qu'offre la chromatine, aux diverses phases de la vie cellulaire. Dans ces derniers temps, un auteur italien, P. Della Valle, a essayé de replacer la question sur ce terrain, qui est le seul véritablement scientifique ${ }^{1}$.

Lécaillon, ont constaté une diversité extrêmement grande des processus de divisıon nucléaire, en particulier des variations énormes dans le nombre des chromosomes. Cela plaiderait contre toute idée de fixité absolue de ce nombre, si les processus en question n'étaient pas manifestement pathologiques et si le développement ne s'arretait pas de très bonne heure. Il n'y a évidemment pas d'opposition directe entre les phénomènes normaux et les phénomènes pathologiques, pas plus en cytologie que dans toutes les branches de la biologie; les deux catégories s'éclairent l'une par l'autre, mais cependant on re peut invoquer la seconde qu'arec beaucoup de prudence pour l'interprétation de la première.

1. P. Della Valle. La Morphologie de ia chromatine au foint de vue physique (Archivio Zoalogiro Italiano, t. 6, 1312.) 


\section{CINQUIEMEE PARTIE}

\section{SEXUALITÉ ET MULTIPLICATION ASEXUÉE}

\section{CHAPITRE XX}

La multiplication asexuée : division (schizogénèse), bourgeonnement (blastogénèse). - Division des Turbellariés et des rers. - Stolonisation des Syllidiens; ses rapports avec la reproduction sexuée. - La reproduction des Cestodes et des Trématodes.

Le bourgeonnement chez les animaux coloniaux. - Polymorphisme; dissociation de l'individualité.

La polyembryonie et la reproduction asexuée.

Lexistence de la reproduction asexuée est corrélative des conditions qui affaiblissent l'individualité.

La reproduction sexuée est le seul mode de propagation des animaux dits supérieurs. Tertébrés, Mollusques, Arthropodes. Mais, dans la plupart des autres embranchements, on rencontre des types plus ou moins nombreux, qui peurent se multiplier aussi par voie asexuée.

Ce mode de reproduction joue alors un rôle assez parallèle à celui de la parthénogénèse dans los cas précédemment étudiés. On doit le considérer comme s'étant établi secondairement, mais il a pris parfois une place plus ou moins grande aux dépens de la 
sexualité. A ce titre, son étude doit être faite sommak sement ici.

$\S 1$ - Division et bourgeonnement. - On range les processus de multiplication asexuée sous deux rubriques principales : la division et le bourgeonnement.

Dans la première (division ou schizogénèse), le corps de l'adulte, avec ses appareils déjà constitués, se partage en deux ou plusieurs fragments, où se complètent, par une rapide régénération, les quelques organes atteints par ce fractionnement.

Dans la seconde (bourgeonnement ou blastogénèse), un ensemble très limité de tissus, parfois seulement un groupe de cellules, devient le siège d'une prolifération active et d'une organogénèse, par laquelle est réalisé un individu nouveau, semblable à celui sur lequel le processus s'est produit.

L'ensemble des tissus proliférateur's ilitiaux conssitue le bourgeon. De son ébauche à son achèvement, 7] parcourt une véritable embryogénie, la blastogénèse, parallèle à celle de l'œuf, ayant mêne terme, mais en différant souvent d'une façon considérable.

De la division au bourgeonnement existent tous les passages et d'ailleurs ce n'est pas le côté morphologique des phénomènes qui nous intéresse ici, mais le zeientissement de l'ensemble de ces deux processus sur la Sexualité. Voyons, à ce point de vue, les cas grincipaux.

Cas des Turbellariés. - Celui qui se présente avec le plus de simplicité est la division, telle qu'on l'observe, par exemple, chez certains Vers inférieurs du groupe des Turbellariés : Microstoma lineare, Stenostoma sieboldi (fig. $35 \mathrm{~A}$ ), ou Cateluna lemnæ. var exemple, espèces fort communes dans nos eaux 
douces. Ce sont des petits animaux aplatis et allongés, sur lesquels on aperçoit toujours des constric-
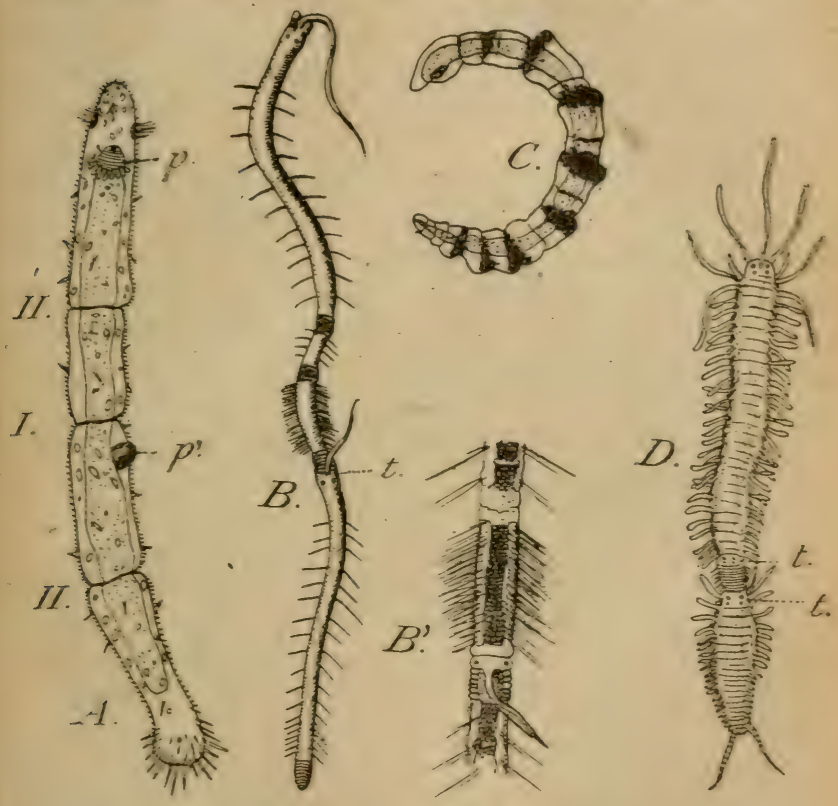

Fia. 35. - Nuelques cas de multiplication aseruée chez les Vers.

A. Stenostoma sieboldi (Turbellarié; Üapres ron Graff); $I$, sillon marquan: une première division en deus in lividus: $I I$, sillon divisant à nouveau chaezne les moitiés; $p$, le pharynx de lindividu souche: $p^{\prime}$ le pharynx deja formé du lourgeon constitué par la moitié postérieure; $B$. Wais (Oitiochètel en voì ds hourgeonnement. On disting"יn plusieurs rizious, ot se formant de nouveaux

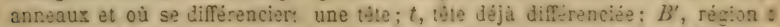
pius @rossie (d'aprés Leuckart). C, Ctenodrilus parialis (dapres heune!), les bandes ombrées indiquent autant de points, où se différenciera une tête. $i$, Syilidien bourgeonnant, dans sa région postérieure, des individus qui se détacheront successivement; $t$, téte des bourgeons arec quatre yeux.

tions transversales régulièrement espacées. En arrière de chacune d'elles, une observation attentive montre 
que sont en train de se constituer les organes caractéristiques de l'extrémité antérieure de l'animal, taches oculaires, pharynx, etc... Peu à peu, les constrictions s'accentuent et le Ver se coupe à leur niveau en individus désormais indépendants, sur lesquels le même processus de division a déjà commencé.

Cas des Annélides Oligochètes. - Tout à fait analogue est la multiplication asexuée, chez diverses Annélides Oligochètes, également d'eau douce, telles que les Dero, les Naïs (fig. $35 B, B^{\prime}$ ), les Chætogaster, les Lumbriculus, etc. Le corps sa compose ici d'un nombre plus ou moins éleré d'anneaux similaires; les premiers sont différenciés en une tête, par la présence de centres nerveux spéciaux, d'organes sensoriels, d'une bouche et parfois de tentacules. A certains niveaux de la chaîne d'anneaux, disposés d'une façon très constante dans chaque espèce, apparaissent les organes caractéristiques d'une tête (fig. $35 \mathrm{~B}^{\prime}$ ); la chaîne d'anneaux est transformée en une chaine d'individus qui se séparent. Quelques Annélides Polychètes, telles que les Salmacines, se divisent de façon analogue.

Tous ces animaux se multiplient ainsi - par voie asexuée - pendant de longues périodes, sans même que, chez beaucoup d'entre eux, apparaisse une ébauche des glandes génitales. Puis, à un certain moment, celles-ci se montrent et généralement alors la reproduction asexuée est suspendue. Il y a, entre celle-ci et la sexualité, une sorte de balancement plus ou moins rigoureux, un cycle plus ou moins régulier, comme nous en arons constaté un entre la parthénogénèse et la gamogénèse.

Ce cycle est parfois réglé sur les saisons. Les Salmacines, par exemple, petites Annélides Polychètes marines qui vivent dans des tubes de calcaire sécrétés 
par elles, bourgeonnent au printemps et se reproduisent sexuellement en été. Mais il est souvent difficile de saisir les causes déterminantes du passage d'un régime à l'autre, bien que diverses études aient déjà été entreprises à cet égard. Chez les Oligochètes cités plus haut, l'apparition des organes génitaux est très irrégulière. Cependant, c'est rers l'automne qu'on trouve plus fréquemment ces formes à l'état sexué. Il est fort difficile, même à ce moment, de trouver des Lumbriculus pourvus des glandes génitales et Mrazek, qui en a observé dans ce but des milliers d'individus, note que les quelques sexués rencontrés par lui offraient une grande variabilité dans la disposition de ces glandes.

La reproduction des Ctenodrilus. - Le genre Ctenodrilus (Annélide rattachée tantôt aux Oligochètes, tantôt aux Polychètes et dont il existe plusieurs espèces) mérite une mention spéciale. Pendant près de quarante ans, il a été obserré, parfois en quantités très considérables, dans de très nombreuses localités et par beaucoup de naturalistes. On en a fait de véritables cultures en aquariums, prolongées pendant plusieurs années. Toujours ce rer se présentait en état d'active multiplication asexuée, comme le montre la fig. $35, C$, où les constrictions indiquent la limite des individus qui ront s'isoler : jamais on ne trouvait aucune trace d'organes génitaux. On pouvait donc considérer, arec beaucoup de rraisemblance, que la reproduction sexuée avait complètement disparu chez cet animal, quand, il y a quelques années, à Naples, daus des sables provenant de la còte du Pausilippe, Monticelli rencontra soudain, en abondance, des Ctenodrilus sexués hermaphrodites. Dans la cavité générale de ces individus, se déreloppaient de nombreux embryons. On ne voit guere 
actuellement quel est le déterminisme de ce retour à la sexualité; il est évidemment fort rare et cet exemple est important, parce qu'il montre arec quelle réserve il faut parler de disparition totale de la reproduction sexuée. Il. en est ici comme de la parthénogénèse permanente et de la disparition totale des mâles. Toutes les données négatives comportent, au reste, la même incertitude 1 .

La stolonisation des Syllidiens. - Les Annélides Polychètes de la famille des Syllidiens sont le siège de processus que l'on peut appeler la stolonisation; ils ont une analogie morphologique indiscutable avec les précédents, tout en ayant une signification physiologique différente. Le corps de ces Vers se fragmente, en effet (fig. $35, D$ ), soit en une fois, soit successivement, en un nombre plus ou moins élevé de troncons, composés d'un certain nombre d'anneaux : l'antérieur acquiert les caractéristiques d'un segment céphalique avec des organes visuels généralement bien développés; les anneaux suivants portent de lungues soies, formant des organes natatoires efficaces, grâce auxquels l'animal se déplace aisément. II y a donc ici multiplication asexuée comme précédemment. Mais les tronçons formés ne peuvent plus se multıplier à nouveau et, en cela, ils diffèrent de ia souche qui les a produits. Ils sont remplis de produits génilaux généralement mủrs ou près de l'être; ils les émcllent au dehors, très peu de temps après leur individualisation et ne tardent pas ensuite à mourir.

1 Un autre organisme, trouvé et cultivẻ uniquement jusqu'ici à l'état asexué, Trichoplax adherens, appartiendrait de meme, d'après Krumbach, à une Méduse (Eleutheria), qui se propagerait ainsi d'une façon pratiquement indéfinie sans reprendre la forme sexuée. Dans ce cas cependant, ces conclusions ont été révoquées en doute. 
Nous n'avons donc pas ici un mode de propagation asexuée de l'espèce, se substituant plus ou moins longtemps et plus ou moins exclusivement à le reproduction sexuée, mais seulement une différenciation de cette dernière, facilitée par la constitution segmentaire ou métamérique du corps des Annélides. Grâce à la fragmeniation de l'animal, au moment de sa maturité sexuelle, la dissémination de ses produits génitaux est plus parfaite et ce phénomène, qui parait, au premier abord, une reproduction asexuée, doit être plutôt considéré comme une complication particulière de la sexualité. L'envisageant ainsi, Giard lui avait donné le nom significatif d'autotomie génératrice ou gonophorique. Au reste, ce n'est que le dernier terme d'une série de transformations; que l'on trouve dans plusieurs familles d'Annélides Polychètes, au moment de la maturité sexuelle et que l'on a désignées du nom d'épitoquie ou épigamiel. Elles consistent dans le renforcement des organes sensoriels, le développement des soies natatoires sur les anneaux du corps, etc..., bref toutes les modifications signalées plus haut, moins la division de l'animal en tronçons indépendants. Celle-ci n'apparait donc que comme une dernière étape des phénomènes d'épigamie, un perfectionnement à la dissémination des produits génitaux, comme le disent très généralement les zoologistes, dans un langage peut-être encore trop finaliste.

Multiplication asexuée chez les Cestodes et les Trématodes. - On peut envisager, d'une façon analogue, les phénomènes de la reproduction, chez les Vers parasites du groupe des Cestodes et des Trématodes.

1. Pour plus de détails sur ces phénomènes, voir Caullear et Messic, les Formes épitoques et l'évolution des Cirratuliens, 1898 (J,-B. Baillière). 
Parmi les premiers, considérons un Tænia. C'est un long ruban formé de centaines et parfois de mil. liers d'anneaux, dont chacun renferme l'ensemble des divers organes. Chaque anneau peut donc être regardé comme un individu sexué; ce serait un hermaphrodite successif, protandrique; car les anneaux ont d'abord des testicules, qui sont mûrs chez ceux qui occupent la région moyenne du ruban; les anneaux terminaux n'ont plus de testicules, mais des ovaires; les anneaux mâles fécondent leurs aînés qui sont à l'état femelle et, dans ceux-ci, auxquels on donne alors le nom de proglottis, les œufs du Tænia se développent en embryons. Successivement les proglottis se détachent, peuvent vivre plus ou moins longtemps libres, puis meurent, mettant en liberté, dans le milieu extérieur, les œufs embryonnés.

$\mathrm{Au}$ lieu de considérer chaque anneau comme un individu, on peut tout aussi bien n'y voir qu'un élément, un segment de l'individu total Tænia, constitué par l'ensemble des anneaux. On a beaucoup discuté sur ces deux conceptions, qui ne sont séparées au fond que par des conventions verbales, sur le sens du mot individu.

Nous sommes là précisément dans un de cescas, où l'individualité ne satisfait plus au double critérium morphologique et physiologique qui permet de la bien délimiter. Si l'on regarde le proglottis comme un individu, la formation des anneaux, ou proglottis, est une multiplication asexuée. Mais, à un autre point de vue, elle peut être interprétée comme le tronçonnement du corps du Tænia, au moment de la maturité génitale, c'est-à-dire une sorte d'autotomie gonophorique, selon l'expression de Giard, précemment rappelée. De toute façon, elle est un processus par lequel la formation et la dissémination continues des embryons sont merveilleusement réalisées. Elle conduit 
à une production énorme de ces embryons, qui est ici une condition importante, sinon indispensable, de la durée de l'espèce. Car, pour derenir un Tænia, l'embryon, inclus dans la coque de l'œuf et rejeté dans le milieu extérieur, devra rencontrer, à points nommés, une série de conjonctures favorables, dont la réalisation successive est des plus problématiques. Aussi la très grande majorité des embryons succombe-t-elle, faute de les trouver, et cette perte considérable de jeunes individus est compensée par leur grand nombre.

Cette nécessité se trouve parfois satisfaite, chez les Cestodes, par un mode différent, qui a plus nettement figure de reproduction asexuée. Il supplée au précédeht, par une sorte de balancement, car on l'observe chez les Tænias, où le nombre et la production des proglottis se trourent très réduits. Avant d'atteindre, l'état rubané, dans le tube digestif de son hôte, le Tænia doit passer par un hôte provisoire, où il est constitué par une résicule plus ou moins grosse, gonflée de liquide, et qu'on appelle le cysticerque (fig. 36). En un point de la surface du cysticerque, s'est différencié ce qu'on est convenu d'appeler la tète du futur Tænia, avec la région où se formeront les anneaux successifs; l'ensemble porte le nom de scolex (fig. 36, $B$, s). Le Tærnia solium, par exemple, qui habite l'intestin de l'Homme, a préalablement été, à l'état de cysticerque, dans les muscles du Porc. Un cysticerque produit, dans la plupart des espèces, un seul scolex et il provient lui-même d'un œuf; un embryon donne donc un seul Tænia. Mais, chez diverses espèces, dont l'état T:enia comporte très peu d'anneaux, le cysticerque donne naissance, non à un seul scolex, mais à un grand nombre, sur toute sa surface. C'est le cas du Cénure, Tænia du Chien, dont le cysticerque (fig. $36, C$ ) se développe de préférence dans le cerveau du Mouton; c'est surtout celui de 
l'Echinocoque. Ce dernier est un minuscule Tænia, long de 4 à 5 millimètres, habitant l'intestin du Chien ou du Chat et in'offrant que trois ou quatre anneaux; mais son cysticerque (fig. $36, D$ ) atteint et dépasse le volume de notre téte et forme, soit dans le foie, soit dans d'autres organes, les redoutables et volumineux kystes hydatiqués. Ici, la résicule qui constitue le

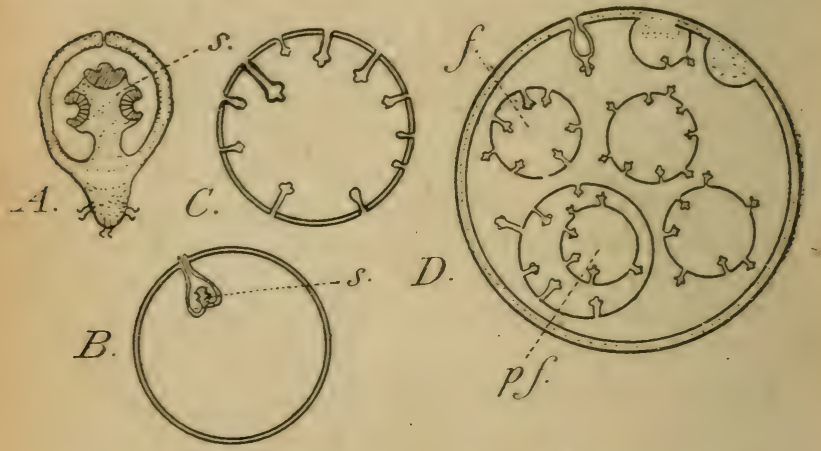

Fig. 36. - Multiplication asexuée des Cestodes.

A, Cysticerque d'un Tænia de la Souris (Hymenolepis murina) montrant le scolex s; ou tête du futur Tænia, avec ses ventouses d'après Grassi et Rovelli); $B$, Cysticerque typique de Tænia (grosse vésicule différenciant à sa périphérie un Scolex $s$ (ex. Tænia solium du porc); C, Cysticerque de Tænia cœnurus (cerveau du Vouton), produisant toute une série de scolex à sa périphérie; $D$, Cysticerque de Tænia Echinocoque (Kyste hy̧datique), produisant des vésicules filles $f$, et celles-ci des vésicules petiles-filles pf, dont chacune forme une série de scolex. (Schẻmas d'après Benham.)

cysticerque est donc énorme et elle produit, par un véritable bourgeonnement à son intérieur, des vésicules-filles $f$; celles-ci, à leur tour, bourgeonnent d'autres résicules, petites-filles de la première $p f$; chacune de ces dernières évolue à la façon d'un Cénure, formant une série de scolex. Un seul cysticerque a donc fourni un nombre énorme de scolex, 
c'est-à-dire de futurs Tænias, et le faible développement de celui-ci se trouve compensé, au point de vue de la propagation de l'espèce, par le très grand nombre d'individus auxquels a donné naissance un seul cysticerque, c'est-à-dire un seul œuf. La production des scolex multiples sur le cysticerque est une reproduction asexuée mieux caractérisée que celle des proglottis dans le Tænia.

Dans d'autres groupes d'animaux parasites, les Trématodes (Douves, etc.), voisins des Cestodes, ou encore chez les Dicyémides et les Orthonectides, à organisation beaucoup plus rudimentaire encore, la propagation de l'espèce est soumise à des vicissitudes analogues. Chez eux aussi la perte d'un nombre très considérable de larves se trouve compensée par une multiplication post-Iarvaire asexuée souvent énorme. Il y a, au cour's des étapes qui précèdent l'état sexué adulte, une ou plusieurs phases successives de multiplication, que je ne puis examiner ici en détail. Dans tous les cas, le résultat est une conservation mieux assurée de l'espèce; mais certains de ces processus, comme ceux que l'on rencontre chez les Dicyémides et les Orthonectides, semblent relever de la reproduction asexuée, tandis que ceux qu'offrent les Trématodes ${ }^{1}$ sont plus probablement des phénomènes de parthénogénèse (donc âe reproduction sexuée), intervenant pendant le développement 2.

\section{§2. - Le bourgeonnement chez les animaux} coloniaux jroprement dits. - Dans tous les cas précédents, la reproduction asexuée ne se montre

1. Formation des rédies dans les sporocystes et des cercaires dans les rédies. (Voir les traités de zoologie et d'embryologie!

2. Parthénogénèse progénétique ou pædogénétique. Cf. la parthénogénèse cies larves de Cécidomyie (Miastor), chap. XIX, ค. 229). 
en quelque sorte que d'une façon épisodique. Elle tient au contraire une place plus régulière et se présente arec un aspect plus net, chez les animaux qui forment des colonies : les Eponges, les Cœelentérés, les Bryozaires et les Tuniciers. Ces groupes ont en commun une caractéristique d'ordre éthologrique, celle de virre fixés à un support 1. L'œuf s'y transforme en un prernier individu qu'on appellera l'oozoide; de celui-ci, par bourgeonnements successifs, naîtront une série de blastozoïdes dont l'ensemble forme une colonie, d'étendue et de complication très variables, suivant les types.

Il n'est pas question d'étudier ici le détail de ces phénomènes, ni même de les passer sommairement en rerue. J'ai seulement pour but d'indiquer leurs rapports avec la reproduction sexuée.

Chez beaucoup de ces animaux coloniaux, il s'établit un polymorphisme entre les blastozoïdes; chacune de leurs formes est en rapport arec le déreloppement particulier d'une fonction. Si cette spécialisation est peu marquée, les diverses catégories sont peu différentes les unes des autres et l'individu est généralement facile à délimiter. Si elle est poussée très loin, comme c'est le cas chez les Siphcinophores, il devient très difficile de dire où commence et où cesse l'individualité, de défmir ce qui a la valeur d'un individu, ou seulement d'un organe. L'individu, dans la vie coloniale, cesse plus ou moins d'ètre un complexe d'organes physiologiquement indivisible. Ce complexe se dissocie et ses fragments, diversement groupés et répétés, concourent à réaliser une individualité

1. Au moins dans les cas qu'on peut considérer comme primitifs. Les exceptions doivent etre considérées comme un retour secondaire à une vie libre, qui d'ailleurs est plutôt un déplacement passif dans le milieu qu'une mobilité active. 
physılogique d'ordre supérieur et elle-même plus ou moins indivisible, la colonie.

Quelle est l'allure de la reproduction sexuée chez les animaux coloniaux? car c'est là le côté par lequel ils nous intéressent ici. D'une façon-générale, elle s'efface au début de la formation de la colonie. L'oozoïde et les premiers blastozoïdes auxquels il donne naissance ne se reproduisent que par voie asexuée; il y a alors blastogénèse exclusive; les individus formés, ou bien ne portent aucun rudiment de glandes génitales, ou bien n'en présentent que des ébauches qui ne parviendront pas à maturité. Il arrive même, comme chez les Botrylles ${ }^{1}$, que les orules, qui ont commencé à mûrir dans un premier blastozoïde, achèvent leur croissance dans une génération ultérieure; et l'on voit ici un exemple nouveau et particulièrement significatif de la dissociation de l'individualité. Les éléments germinaux n'appartiennent plus en propre à un individu, mais à la colonie.

Très souvent aussi, dans ces divers groupes, la reproduction sexuée est dévolue d'une façon exclusive à certains individus spéciaux - appèlés les gonozoïdes - alors que tous les autres sont stériles, dépourvus de toute ébauche germinale. Chez les Cælentérés, la Méduse, parfaite ou imparfaite, est le gonozoïde, et les polypes ordinaires de la colonie restent complètement asexués.

La polyembryonie enfin 2 constitue une dernière catégorie de faits, pouvant rentrer dans la multiplication asexuée et on peut noter que, chez presque toutes İes espèces où on l'a constatée, l'œuf, qui se divise en embryons multiples, vit à la façon d'un parasite, soit dans un autre animal, soit greffé sur l'organisme

1. Ascidies composées (Tuniciers,.

2. Voir chap. XII, p. 152. 
maternel. Le phénomène est donc encore corrélatif d'un mode d'éthologie qui, d'une façon générale, affaiblit la netteté de l'individualité. Ce cas se rattache donc assez naturellement aux autres, par les circonstances où il se produit.

§ 3. - La multiplication asexuée est corrélative de l'effacement de l'individualité. - En somme, la reproduction asexuée apparait dans les cas où l'individualité s'efface. Chez les animaux, celle-ci, comme nous l'arons déjà dit, résulte normalement d'une double caractéristique, l'une morphologique, l'autre physiologique. Morphologiquement, tous les tissus dérivant de l'œuf forment un ensemble indivisible et indissoluble; physiologiquement, chacun des organes composants est strictement indispensable à la vie du tout.

L'organisme, de par cette étroite solidarité fonctionnelle des parties, ne peut pas subir des pertes étendues dans ses œurres vires, ni les réparer. Le pouvoir régénérateur est limité.

Certaines conditions éthologiques, dont le parasitisme et la fixation sont les principales, ou bien une constitution morphologique comprenant la répétition d'unités équivalentes (comme les anneaux d'une Annélide), diminuent la solidarité des parties de l'organisme, lui permettent de supporter des pertes étendues, ce qui s'allie, en fait, au pouvoir de les réparer par la régénération.

La multiplication asexuée me paraît n'être à l'origine que la régularisation de ce pouvoir régénérateur, la trunsformation d'un processus accidentel de réparation, en un"processus physiologique et normal de propagation. Cette possibilité existant, elle s'est développée considérablement et a abouti áux résultats résumés ci-dessus, en entraînant, pour la 
sexualité, les conséquences que nous arons vues. Mais, contrairement à ce que pensent certains biologistes, je suis fermement convaincu que, chez les animaux, la multiplication asexuée, telle que nous la constatons actuellement, est, comme la parthénogénèse, une propriété secondairement acquise, et non pas le restige d'un mode fondamental primitif et général de multiplication des organismes. Certes, les premiers êtres pluricellulaires ont pu, de par la simplicité et l'indifférenciation probable de leur constitution, posséder la reproduction asexuée; mais, dans les groupes où nous la retrouvons aujourd'hui, il me semble qu'elle a dû reparaitre, après avoir disparu. Tous ces animaux, en effet, appartiennent à de rastes groupes, où la reproduction sexuée existe seule et. a été vraisemblablement seule héritée des ancêtres immédiats.

Les conditions éthologiques qui font reparaitre la multiplication asexuée et la dissociation de l'individualité - la fixation est la principale - rapprochent. l'animal du régétal, en réduisant les fonctions de relation qui manquent à ce dernier. Toutes les transformations morphologiques ou physiologiques que subit de ce fait l'animal sont de mème ordre que celles que nous présente le végétal. Lá aussi l'individualité est difficile à définir, la solidarité des parties moindre, la faculté de supporter des pertes et de les réparer très grande. Dans bien des particularités que je ne puis développer ici, l'animal colonial, Coelentéré Bryozoaire ou Tunicier, a le faciès, l'allure et le mode de réaction du régétal. La colonie se comporte plus ou moins comme la plante. La sexualité de la colonie, si on réfléchit, montre aussi d'étroites analogies avec celle de la plante, notamment dans le moment de son apparition et les conditions histologiques de sa réalisation. Dans un cas comme dans l'autre, en 
mème temps que l'indiridualité s'estompait et s'effaçait, de par les conséquences de la fixation au substratum, la multiplication asexuée a repris une importance comparable à la reproduction sexuée, et parfois même l'a plus ou moins complètement supplantée. 


\section{SIXIÈME PARTIE}

\section{LA SEXUALITÉ GHEZ LES VÉGÉTAUX ET LES ORGANISMES INFÉRIEURS (PROTOZOAIRES ET THALLOPHYTES)}

Dans tous les chapitres précédents, c'est surtout la sexualité des animaux pluricellulaires, des Métazoaires, qui a été étudiée. Elle y présente en somme une remarquable homogénéité, dans ses traits essentiels. Ovule et spermatozoïde, considérés isolément ou dans leurs rapports mutuels, sont constitués et se comportent de façon très semblable, qu'il s'agisse d'une Éponge, d'un Insecte ou d'un Vertébré. Cette similitude se poursuit, ainsi que nous l'avons vu, dans le mode de formation des gamètes, dans la spermatogénèse et dans l'ovogénèse.

Pour avoir une idée des phénomènes de la sexualité dans l'ensemble de tous les organismes, il y aurait à les étudier aussi chez les végétaux et chez les animaux unicellulaires ou Protozoaires. Il conviendrait de traiter en même temps que ces derniers les végétaux inférieurs ou Thallophytes, c'est-à-dire les Champignons et les Algues, et, d'autre part, les végétaux supérieurs aux Thallophytes, c'est-à-dire les Mousses et Hépatiques, les Cryptogames varculaires 
(Fougères et groupes qui s'y rattachent) et les Phanérogames.

L'ordre logique aurait même consisté à partir des formes les plus simples, Protozoaires et Thallophytes, à y chercher les premières ébauches de la sexualité et à retracer comment, de ces rudiments initiaux, a dérivé, conformément aux lois de l'évolution, la Sexualité, telle que nous la trourons chez les organismes supérieurs.

Je n'ai pas cru, je l'ai dit au début de ce livre, deroir procéder ainsi, quelles que fussent les raisons théoriques qui pussent m'y pousser.

Pour être traité avec la précision nécessaire, le sujet ainsi compris aurait demandé une place et une illustration trop considérables et il aurait exigé des connaissances trop complètes sur la morphologie et la classification des Protozoaires et de Thallophytes; il eût entrainé à des discussions trop savantes. J'ai donc pensé préférable de me borner à jeter un coup d'œil rapide, en terminant, sur les divers groupes en question, en me limitant aux données principales. Le lecteur trouvera ainsi, dans cette dernière partie, un aperçu sur cette raste question.

Pour les mêmes raisons d'ordre pratique, j'examinerai successivement les Végétaux Cryptogames, puis les Phanérogames et enfin les Protozoaires et les Thallophytes ${ }^{1}$.

1. Voir, pour plus de détails sur la Sexualité des végétaux, G. Bonxier, le Monde végétal (Bibliothèque de Philosophie Scientifique). 


\section{¿HAPITRE XXI}

\section{LA SEXUALITE CHEZ LES VÉGÉTAUX ${ }^{1}$}

.. - Archégoniées : Archégone et anthéridie. - Fougères : plante feuillée et prothalle; sporophyte et gamétophyte (gẻnération diploïde et génération haploïde). - Alternance de générations. - La réduction chromatique a lieu lors de la formation du gamétophyte et non lors de la formation des gamètes.

II. - Phanérogames : Les gamètes et la fécondation. - Le grain de pollen est un prothalle mâle rudimentaire. - Anthérozoïdes du Cycas et du Gingko. - La transformation du gamète mâle est corrélative de ce que la fécondation s'accomplit à sec. - Régression du prothalle femelle des Phanérogames. - Cas transitionnel des Gymnospermes. - Les Ptéridospermées fossiles.

$\S 1$ - Archégoniées. - On groupe sous le nom d'Archégoniées (dont le sens apparaitra immédiatement) les Mousses et les Cryptogames vasculaires (Fougères, Prêles, Lycopodes): tous ces végétaux ont des gamètes qui rappellent, à bien des égards, ceux des animaux.

Le gamète femelle, équivalent de l'ovule animal, est désigné généralement sous le nom d'oosphère; il est situé dans un appareil en forme de bouteille, l'archégone (fig. 37, A, B). Il en occupe la partie nrofonde et renflée, qui est surmontée d'un col plus

\section{Autres que les Thallophytes.}


ou moins long, traversé par un canal, où s'engage l'élément mâle, pour venir le féconder.

Les gamètes mâles, ou anthérozö̈des (fig. 37, C-E), sont des cellules produites dans un organe appelé l'anthéridie. Ils sont constitués d'une façon tout à

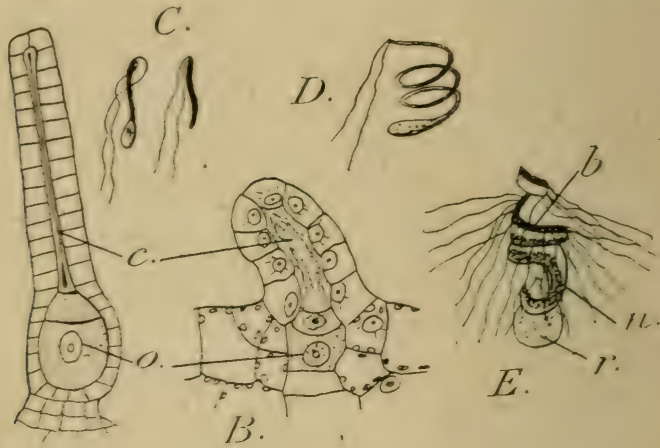

Fig. 37.

Archégones : $A$, dune Hepatique Murchantia); $B$, dune fougère (Polypodium); $c$, canai; 0 , gamète femelle (oosphère).

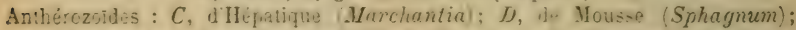
$E$, de Fougiere (frmnogramne). Sur ce derrier, gui est spiralé, on voit de noml reux cils insidis sur une bande tres colorable, le bléfharoplaste b homojogue du centwosome lans les spermatcizoides): $n$, noviu (rubané); $r$, le reste du crtaysme inutilisé de la spermatile. Daprès Schenck, Schimpier et Belajeff.)

fait analogue au spermatozoïde animal. Ce sont, en effet, des éléments longs et minces, généralement enroulés en spirale; presque toute leur masse est constituée par le noyau, étiré comme le corps de l'anthérozoïde; ils portent des cils ou flagelles, gràce auxquels ils ont une grande mobilité dans un milieu liquide.

Oosphère et anthérozoïde sont donc différenciés, l'un par rapport à l'autre, comme l'ovule et le sper- 
matozoïde. Les Archégoniées nous montrent ainsi la même anisogamie que les Métazoaires.

La genèse de ces gamètes présente toutefois, par rapport à ces derniers, une différence qu'il convient de signaler. Chez les animaux, la formation des spermatozoïdes, ou la maturité de l'ovule, est précédée immédiatement de deux divisions cellulaires où le nombre des chromosomes est réduit de moitié. C'est ce que nous avons appelé la phase méiotique; elle termine l'ovogénèse et la spermatogénèse, et elle apparait comme le prélude nécessaire et immédiat de la fécondation. Chez les Archégoniées, il n'en est pas tout à fait de même.

La plante parcourt un cycle qui comprend deux phases généralement bien distinctes ou, si l'on veut, deux générations alternant régulièrement. Nous en aurons une idée en considérant les Fougères.

Cycle d'une Fougère. - Sous les feuilles des Fougères, que tout le monde connait, on aperçoit, à certains moments, des organes saillants, les sores, renfermant des loges, ou sporanges, dans lesquels se forment un grand nombre de petits germes asexués, les spores. La Fougère feuillée ne présente jamais d'organes sexuels; c'est un appareil asexué ou, comme on dit, un sporophyte. Chaque spore, en germant sur le sol humide, donne naissance à une petite plante, d'organisation très simple, très différente et beaucoup plus difficile à apercevoir, le prothalle. Sur ce prothalle, se forment des organes sexués, les archégones et les anthéridies. Le prothalle est donc une plante sexuée, portant les gamètes, un gamétophyte. Les anthérozoïdes vont y féconder les oosphères et chaque œuf ainsi produit sur le prothalle, en se déreloppant, devient la plante feuillée, ou sporophyte, dont nous sommes partis. Le cycle d'une Fougère se com- 
pose donc de la succession alternative de deux appareils ou, si l'on reut, de deux générations, le sporophyte asexué et le gamétophyte sexué 1 .

Or, les recherches de Strasburger et d'une série d'autres botanistes ont montré que ces deux générations différaient par les noyaux de leurs divers tissus. Le nombre des chromosomes dans les noyaux du gamétophyte est la moitié de celui qu'on trouve dans ceux du sporophyte 2 . Si le premier est $n$, le second est $2 n$.

La réduction du nombre des chromosomes ne se fait pas au moment de la formation des gamètes, c'est-à-dire immédiatement arant la fécondation, comme chez les animaux. Elle a eu lieu antérieurement, lors des deux dernières divisions cellulaires qui, sur le sporophyte, précèdent la formation des spores; ces divisions offrent des particularités tout à fait parallèles à la méiose, que nous avons décrite précédemment. La phase méiotique se place donc ici à la formation du gamétophyte, au lieu d'être à la formation des gamètes. Telles sont, abstraction faite de tout examen détaillé, les conditions essentielles de la sexualité chez les Archégoniées. J'ajouterai seulement que, dans les Mousses, la plante que tout le monde connait est, non pas le sporophyte comme chez les Fougères, mais le gamétophyte. Le sporophyte n'a pas ici une existence indépendante; il se développe sur le gamétophyte même et se réduit au

1. Des processus de multiplication asexuée permettent à certaines Archégoniées de se perpétuer plus ou moins longtemps, - pour certaines presque indéfiniment, - sans passer par la génération sexuée. On trouve des exemples du méme ordre chez les animaux (v. chap. XX). D'autre part, diverses Fougères montrent aussi de la parthénogénèse.

2. D'où le nom de génération haploïde (ä àiovę simple), donné au gamétophyte et de génération diploide (ôt le sporophyte. 
pédicelle terminé par une capsule remplie de spores. Les deux phases du cycle sont bien réelles encore, mais s'accomplissent sur une mème plante.

§2. - Phanérogames. - Les gamètes et les conditions de leur formation, chez les Phanérogames, diffèrent beaucoup, en apparence, du cas des Archégoniées. Cependant on a pu, peu à peu, les ramener à celui-ci et reconstituer, dans leur histoire, un des plus beaux exemples de l'évolution d'un organe et d'une fonction, au cours de la phylogénie.

Le cycle d'une Phanérogame ne comporte plus deux générations successires, l'une asexuée, l'autre sexuée, mais une seule plante, sur laquelle se forment les gamètes, dans les fleurs. Les éléments mảles sont les grains de pollen, produits en nombre considérable dans les étamines. Ils n'ont pas de mobilité propre, mais doivent être passivement transportés par des agents dirers : le rent, les Insectes ou d'autres moyens, jusqu'à l'organe femelle. Celui-ci, le pistil, la partie la plus interne de la fleur, est formé de feuilles modifiées, les carpelles, qui portent les cellules femelles, à leur base, au sein d'organes complexes; leur partie supérieure, étirée en un long col, le style, se termine par une sorte de papille, le stigmate, où viennent tomber les grains de pollen. Sur le style et le stigmate, un tissu particulier produit un liquide sucré; sous l'influence nutritive de ce liquide, les grains de pollen émettent un prolongement cytoplasmique, le tube pollinique, qui s'allonge sur le style et parvient ainsi, à la base des carpelles, jusqu'au contact du gamète femelle, l'oosphère ${ }^{1}$. Celle-ci

1. L'oosphère est inclus dans un massif cellulaire complexe qui deviendra la graine et pour lequel l'usage a consacré le nom d'ovule. Ce mot n'a donc malheureusement pas le même sens, en zoologie et en botanique et cela est très regrettable, car 
est l'une des huit cellules constituant un appareil arpelé le sac embryonnaire (fig. $38 \mathrm{se}$ ) et qui s'est différencié sous l'épiderme du carpelle.

On ne trouve donc pas ici, au moins au premier abord, l'équivalent de l'archégone, ni l'anthérozoïde

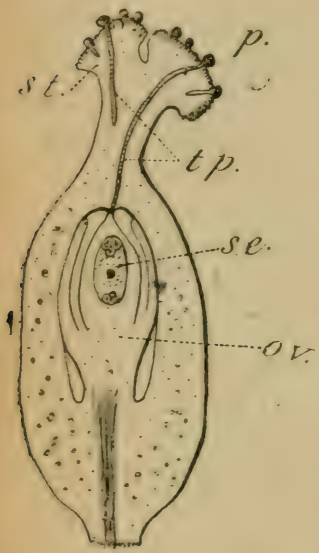

Fra. 38. - Coupe du pistil de Palygoaum convolxulus, au moment de la fécondation. Plusieurs grains de pollen $p$, tombeis sur le stigmate $s t$, germent, er woyant des tubes polliniques $t p$, vers l'ovule ov; se, sac embryonnaire (d'après Schenck). cilié qui rappelait si nettement le spermatozoïde animal. L'élément mâle a perdu toute motilité propre. Il ne reste d'analogie, avec les cas examinés précédemment, que la différence entre les deux gamètes, l'anisogamie. Par contre, en étudiant les divisions cellulaires qui aboutissent aux gamètes, on trouve que, comme chez les animaux, il y a réduction du nombre des chromosomes à moitié, lors des divisions qui précèdent immédiatement la formation des grains de pollen ou la différenciation du sac embryonnaire.

Le grain de pollen est un prothalle male. - Mais, en réalité, le grain de pollen (fig. 40, p. 279) n'est pas, dans son ensemble, le gamète màle. Il renferme deux noyaux au moins: l'un d'eux ne joue aucun rôle dans la

c'est une source de confusion. Je m'en suis tenu ici à l'usage, malgré qu'il soit illogique; mais il serait à souhaiter qu'on fit cesser cette ambiguité : soit que les zoologistes renoncent au mot orule et le remplacent par oosphère, soif que les botanistes introduisent un terme nouveau au lieu d'ovule dans la description du pistil 
Pécondation et doit être appelé le noyau végétatif $n v$, l'autre étant le noyau générateur $n g$. Ces noyaux s'engagent dans le tube pollinique; le noyau générateur s'y divise en deux, $n g^{\prime}$ et $n g^{\prime \prime}$. L'un de ces deux noyaux, $n g^{\prime}$, se fusionne avec le noyau de l'oosphère, pour former le noyau de l'œuf, origine de l'embryon : c'est le noyau fécondateur proprement dit. L'autre, $n g^{\prime \prime}$, vient se fusionner avec un autre des huit noyaux du sac embryonnaire et former un autre zygote, origine d'un tissu, l'albumen, dont se nourrira l'embryon. Les gamètes mâles proprement dits sont donc les deux noyaux $n g^{\prime}$ et $n g^{\prime \prime}$ du tube pollinique, et il y a, ainsí que l'ont découvert, en 1899, Nawaschine et Guignard, chez les Phanérogames, une double amphimixie (fig. 39) : de l'une résulte l'embryon, de l'autre l'albumen, aux dépens duquel le premier se comporte comme un parasite.

La fécondation des Phanérogames a ainsi une allure toute particulière et qui ne semble avoir aucun lien direct avec elle des animaux et des Archégoniées. Elle n'est cependant qu'une modification des processus existant dans

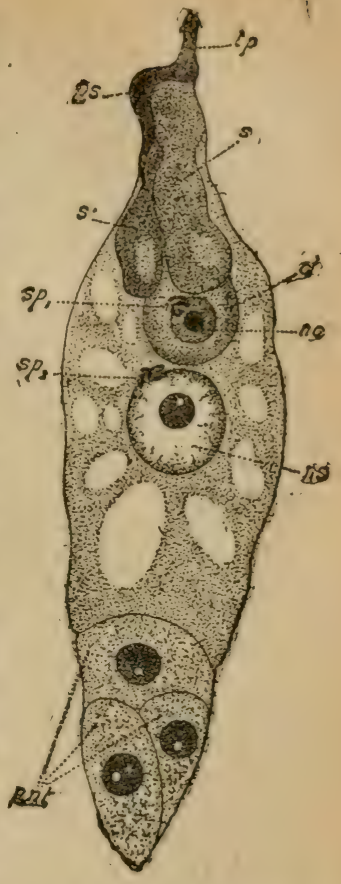

Fig. 39. - Double amphimixie, dans le sac embryonnaire du Tnpinambour (d'après Nawaschine).

$t p$, rube pollinique; $s, s^{\prime}$, synergides; ant, antipodes; $s p_{1}, s p_{\text {s. }}$ les deux anthérozoildes provenant du tube pollinique, et se fusionnant : $s p$, avec le noyau du gamète femelle proprement dit, ou oosphère (pour fortner l'œut); $s p_{2}$ avec le noyau secondaire $n s$ du sac embryonnaire (pour former l'albumen). 
ces dernières; modification profonde, il est vrai, mais dont les botanistes ont réussi récemment à reconstituer toutes les étapes.

Les deux noyaux fécondateurs $n g^{\prime}$ et $n g^{\prime \prime}$ du tube poilinique sont, en effet, des anthérozoïdes, dont l'appareil moteur, les flagelles, ont disparu, corrélativement aux circonstances de la fécondation des Phanérogames : le gamète màle, en effet, n'a plus de trajet à accomplir dans le milieu extérieur pour atteindre l'élément femelle. On a saisi la trace de cette évolution, en étudiant des Phanérogames, que l'ensemble de leur organisation et la structure de l'appareil floral indiquent comme les moins différenciées, les Gymnospermes ${ }^{1}$.

G'est ce qu'ont découvert, de 1897 à 1900, Ikeno et Webber, sur les Cycadées (Zamia, Cycas), et Hirase et Webber, sur un genre de Conifères, le Gingko. Prenons comme exemple le Zamia. Les grains de pollen viennent, comme chez toutes les Phanérogames, germer sur la fleur femelle et y pousser leur tube pollinique. Ici, on peut reconnaître en celui-ci un prothalle rudimentaire; de plus, autour des noyaux $n g^{\prime}$ et $n g^{\prime \prime}$ (correspondant à ceux que nous avons désignés de la même façon chez les Angiospermes), se délimitent deux cellules piriformes, qui présentent, au voisinage de l'une de leurs extrémités, une spirale de courts flagelles (fig. $40 \mathrm{G}$ ). L'étude cytologique minutieuse de la formation de ces éléments montre leur identité arec des anthérozoïdes, dont ils ont gardé l'appareil moteur, les cils; mais ces cils ne se forment plus

1. Ce sont en meme temps les premières à apparaître dans l'histoire du globe, d'après les données actuelles de la paléontologie, puisqu'on les trouve déjà, authentiquement, à la fin de l'époque primaire, alors que les Angiospermes (Monocotylédones et Dicotylédones) ne se montrent que vers la fin du Jurassique. 
qu'au moment où le tube pollinique est arrivé au contact de l'appareil femelle et où la fécondation proprement dite va s'opérer. Ainsi le tube pollinique des Cycadées et des Gingko produit deux véritables anthérozoïdes, et les deux noyaux $n g^{\prime}$ et $n g^{\prime \prime}$, que nous retrouvons chez les autres Gymospermes et chez les
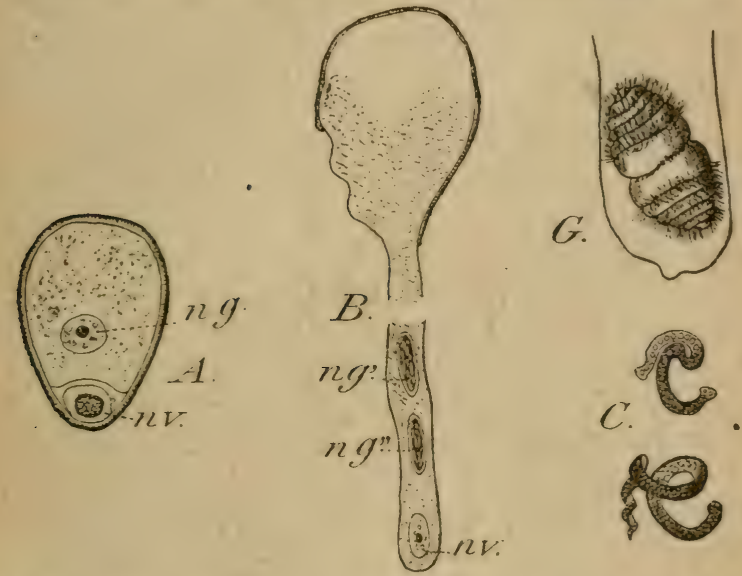

Fic. 40. - $A$, un grain de pollen (de Lys martagon), $n v$, noyau végétatif; $n g$, noyau générateur. $B$, grain de pollen ayant germé, avec ie tube pollinique (sectionné) : $n v$, noyau végétatif qui s'est porté à l'extrémité du tube pollinique; $n g^{\prime}, n g^{\prime \prime}$ noyaux résultant de la division du noyau générateur et qui formeront les deux anthérozoides. (Schémas d'après Schenck.) C', les deux anthérozoides du Topinambour au moment de la fécondation (cf. fig. $39 \mathrm{sp}$, $s p_{3}$ ), (d'après Nawaschine). $G$, les deux anthérozoides du Zamia (avec une spirale de cils), à l'extrémité distale du tube pollinique.

Angiospermes, représentent deux anthérozoïdes qui ne différencient plus d'appareil ciliaire.

Dès lors, il apparaît clairement que le grain de pollen, dans son ensemble, n'a pas la valeur d'un simple gamète, mais bien d'un prothalle mâle rudimentaire, qui, au lieu de produire un grand nombre 
d'anthérozoïdes, n'en produit plus que deux. L'anthérozoïde des Archégoniées était une cellule mobile, organisée pour sc déplacer librement à la recherche de l'archégone en milieu liquide, condition toujours réalisée, étant donné la façon dont régète le prothalle des Archégoniées. Les conditions où se fait la fécondation des Phanérogames, dans une fleur aérienne, excluent d'une façon normale la présence de l'eau. Le prothalle mâle de ces plantes est adapté à une fécondation à sec, au contact de la partie femelle.

Les anthérozoïdes ont dû perdre graduellement, chez ces plantes, leur organisation d'éléments mobiles. Le prothalle mâle tout entier a été transporté au contact de l'appareil femelle et les anthérozoïdes n'ayant plus eu aucun trajet à effectuer par euxmêmes, leur appareil moteur a dégénéré; il n'a subsisté d'eux que l'élément essentiel, au point de vue de la fécondation amphimixique, le noyau. Les Cycadées et le Gingko nous montrent, pour l'appareil mâle, la transition du prothalle au grain de pollen. des Phanérogames; le souvenir des anthérozoïdes mobiles ne s'y est pas encore effacé 1 .

Ainsi, le pollen de Phanérogames est le reste du prothalle mâle, et, dès lors, on comprend très bien que la réduction chromatique ait lieu, lors des deux divisions, qui précèdent l'individualisation des grains de pollen, comme elle avait lieu, chez les Archégoniées, à la formation des spores; c'est dans les deux cas, à l'origine du prothalle ou gamétophyte.

1. La réduction à deux du nombre des anthérozoïdes produits par un grain de pollen, c'est-à-dire par un prothalle, est corrélative du fait que ces anthérozoïdes arrivent sûrement à l'appareil femelle. On voit ainsi constamment, dans la Nature, le nombre des éléments (ou des embryons) produits s'exagérer ou se réduire, parallèlement aux chances de perte ou de survie, qu'ils ont dans les conditions où ils sont placés. 
En dépit des dissemblances, les Phanérogames et les Archégoniées ont mème appareil générateur mâle. Le gamétophyte mâle, chez les premières, est seulement devenu rudimentaire et a pris la forme de grain de pollen.

Vestiges du prothalle femelle. - De même, l'appareil femelle des Phanérogames résulte de la transformation et de la réduction progressive du prothalle femelle, et, dans la nature actuelle, les Gymnospermes nous montrent, comme pour l'autre sexe, une phase transitionnelle, ainsi qu'on le sait depuis assez longtemps.

Les huit cellules du sac embryonnaire et deux ou trois cellules sœurs représentent, chez les Angiospermes, le prothalle femelle tout à fait rudimentaire. Chez les Gymnospermes, ce prothalle constitue encore tout un tissu sous-épidermique du carpelle, que les botanistes ont appelé l'endosperme. Il s'y différencie un certain nombre d'appareils, appelés corpuscules, et qui ne sont autres que des archégones typiques. Ainsi l'endosperme nous apparait comme un prothalle femelle; c'est un gamétophyte qui, au lieu d'ètre une petite plante indépendante, à la façon de celui des Archégoniées, végète sur le sporophyte qui l'a produit et s'y réduit à un massif de cellules. Le nombre des chromosomes, dans les noyaux des cellules de l'endosperme, est réduit de moitié, par rapport aux autres tissus de la plante, et cela se conçoit aisément, car, chez les Archégoniées, cette réduction avait lieu lors de la formation des spores sur le sporophyte, c'est-à-dire à l'origine du gamétophyte; cela correspond bien au début de la différenciation de l'endosperme chez les Gymnospermes.

Ainsi, comme l'appareil màle, l'appareil femelle des Phanérogames les plus élevées dérive directement 
de celui des Archégoniées. La trace de l'alternance de deux générations asexuée et sexuée, sporophyte et gamétophyte, subsiste dans l'unique appareil végétatif qui compose le cycle des Phanérogames. Le gamétophyte s'est réduit à un rudiment, qui a perdu son autonomie et a végété sur le sporophyte même. Des Mousses aux Angiospermes, nous royons l'appareil et les éléments sexuels garder la même signification morphologique, mais nous assistons à la réduction progressive du gamétophyte et à la prépondérance croissante et enfin exclusive du sporophyte.

Cet ensemble de données a encore été très heureusement complété, au cours des dernières années, par des découvertes importantes faites dans le domaine de la Paléontologie végétale. Toute une série de plantes de l'époque primaire, en particulier de l'époque houillère, avaient d'abord été considérées comme des Fougères, parce que leurs frondes, seules connues tout d'abord, ressemblaient étroitement à ce groupe (ex. : les genres Pecopteris, Nevropteris, Odontopteris, etc.). Elles rappelaient, jusque dans le détail de la découpure des feuilles, divers genres de Fougères vivantes. Plus tard, on vit qu'il fallait rapporter à ces frondes, en forme de Fougères; des tiges offrant des caractères anatomiques de Cycadées, et l'on en fit un groupe spécial, participant à la fois des Cycadées et des Fougères, les Cycadofilicinées. Il y a dix ans, on a trouvé, outre les frondes stériles, celles qui portaient l'appareil reproducteur et on a constaté que ces plantes, à aspect de Fougères et à caractères anatomiques de Cycadées, avaient de véritables graines comme les Phanérogames; seulement ces graines se formaient, non dans des fleurs différenciées, mais sur les feuilles mêmes. On assiste évidemment chez ces plantes (aujourd'hui désignées sous le nom de Ptéri- 
dospermées), qui ont completement disparu depuis le commencement des temps secondaires, à la transfor. mation d'un prothalle autonome germant sur le sol, en un prothalle régétant sur la feuille mème, où est née la spore qui lui a donné naissance. Quand bien mème les Ptéridospermées ne seraient pas les plantes par lesquelles s'est fait effectirement le passage des Cryptogames rasculaires aux Gymnospermes, elles nous montrent comment ce passage a pu ètre réalisé.

Nous avons donc ainsi, sous les yeux, soit dans la nature actuelle, soit dans les régétaux fossiles, une admirable série de transformations de l'appareil sexuel et des gamètes, un des plus merreilleux exemples d'érolution d'un appareil et d'une fonction. 


\section{CHAPITRE XXII}

\section{LA SEXUALITÉ CHEZ LES PROTOZOAIRES}

Diversité des phénomènes de sexualité chez les organismes infẻrieurs.

La sexualité des Infusoires. - Travaux d'E. Maupas : le rajeunissement caryogamique. - Isogamie et anisogamie. Recherches de Calkins, Enriques, Woodruff. - La sexualité chez les autres Protozoaires; les conditions de sa manifestation. - Les Protozoaires parasites (Coccidies, Hématozoaires, etc.).

L'autogamie chez les Protozoaires.

Il semblerait, a priori, que chez les organismes inférieurs, Protozoaires et Thallophytes, les phénomènes de la sexualité dussent être rudimentaires et que leur analyse fût aisée. C'est bien l'opinion qu'on avait il y a vingt-cinq ans encore, mais elle a dû être de plus en plus modifiée, au fur et à mesure que la connaissance de ces êtres a progressé.

Qu'on considère les gamètes eux-mêmes, oư les conditions dans lesquelles se manifeste la sexualité, les phénomènes se présentent chez ces êtres inférieurs avec une très grande diversité; ils atteignent, chez beaucoup d'entre eux, un degré de différenciation au moins égal à ce que nous offrent les Métazoaires ou les Végétaux supérieurs, et leur déterminisme est aussi complexe.

Sans doute nous trourerons des types chez lesquels 
la sexualité n'est encore qu'à l'état d'ébauche, et, en comparant des séries de formes convenablement choisies, nous pourrons nous représenter comment la différenciation de la sexualité s'est effectuée peu à peu; mais cette différenciation s'est produite d'une façon parallèle et indépendante, dans de très nombreux groupes, c'est-à-dire d'une façon polyphylé tique.

L'étude de la sexualité dans les Thallophytes et les Protistes, si l'on sort des généralités, exigerait donc de longs développements et l'examen particulier de nombreux cas, que l'on ne peut entreprendre ici. Je me bornerai donc à quelques exemples significatifs.

Considérons d'abord les êtres unicellulaires, Protozoaires ou Algues. Ils se multiplient par division : soit en deux, soit en un nombre plus ou moins élevé de fragments, et ce mode de reproduction asexué peut se poursuivre pendant plus ou moins longtemps, peut-être inc' niment.

\$1. - La sexualité des Infusoires. - C'est ce qui a lieu chez les Infusoires, Protozoaires dont le corps est couvert de cils vibratiles, tels que les Paramécies (fig. 41). Mais à un moment donné, deux individus semblables s'accolent longitudinalement, nagent, ainsi soudés, pendant plusieurs heures, puis se séparent. C'est ce qu'on a appelé la conjugaison. Or, pendant et après la conjugaison, s'accomplissent des phénomènes très caractéristiques, dans l'appareil nucléaire, qui, chez tout Infusoire, comprend deux noyaux, l'un grand (macronucleus), l'autre petit (micronucleus). Le macronucleus, qui paraît présider à la vie végétative, pendant les périodes de multiplication ordinaire, dégénère à la suite de la conjugaison. Quant au micronucleus, il subit plusieurs divisions, chez les deux individus qui viennent de se conjuguer; et - sans que 
je reuille ici exposer les faits en détail - un des noyaux formés ainsi dans chaque individu passe dans l'autre. où il se fusionne avec l'élément correspondant autochtone. Il se constitue, de la sorte, dans chaque

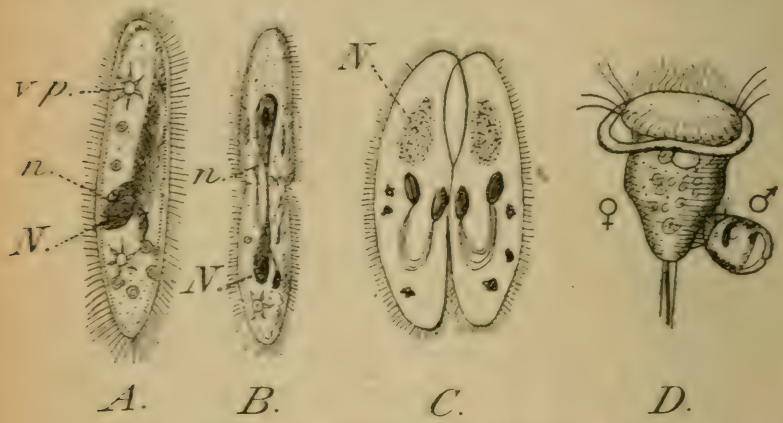

Fi6. 41. - Conjugaison des Infusoires.

$A$, Paramécie; $B$, la méme en roie de division; $C$, deux Paramécies en conjugaison; $D$, Vorticeiles en conjugaison $\left(0^{\circ}\right.$, microgamete; O, macrogamète); $N$, macronucleus; $n$, micronucleus; $v p$, vacuole pulsatile. Dans la fig. $C$. le micronucleus s'est divisé deux fois; trois des noyaux formés dégénérent et le $4^{\circ}$ achève de se diviser à nouveau en deux, formant une figure en haltère.

individu, au, cours de la conjugaison, un noyau zygotique, mixte par ses origines, l sut comme l'est le noyau de l'œuf fécondé.

La conjugaison comporte donc un mélange de substances nucléaires des individus conjugués, ou si l'on veut une amphimixie nucléaire. Le noyau mixte, qui a élé ainsi réalisé, dans chaque conjuguant, se dirise ensuite et c'est lui qui donne naissance au micronucleus et au nouveau macronucleus des deux Infusoires, lorsqu'ils se séparent. Cette amphimixie a done eu pour résultat la refonte complète de l'appareil nucléaire de l'Infusoire. Elle est une fécondation réciproque des deux individus conjugués. 
Le rajeunissement caryogamique (Maupas). - Nous devons la connaissance précise et détaillée de ces phénomènes si complexes à divers auteurs et, arant tout, à E. Maupas. Cet auteur a observé que la conjugaison se produisait surtout dans des cultures, où la multiplication avait longtemps duré (après 200 à 300 divisions consécutives) et qui donnaient des signes d'épuisement, de sénescence. Après la conjugaison, au contraire, les Infusoires, qui se sont séparés, sont aptes à fournir une nouvelle culture vigoureuse et à multiplication intense : ils sont rajeunis. Maupas attribue, avec beaucoup de raison, leur vigueur nouvelle à la refonte de leur appareil nucléaire par l'amphimixie, aussi interprète-t-il celle-ci comme un rajeunissement caryogamique.

Les deux Infusoires qui se conjuguent nous apparaissent donc comme deux gamètes; mais ils ne se fusionnent pas, et ils sont semblables entre eux : il y a isogamie.

Dans ce même groupe des Infusoires, nous trourons des exemples où les gamètes sont inégaux; il y a anisogamie. L'un d'eux, ou microgamète, est plus petit, libre, mobile; il va à la recherche de l'autre, le macrogamète, plus gros et fixe; la substance du microgamète se fusionne en partie avec celle du macrogamète et le reste dégénère. C'est ce que montrent les Vorticelles (fig. $41 D$ ). Nous trouvons done ici la sexualité, sous une forme tout à fait semblable à ce que présentent les Métazoaires. Ainsi, sans sortir du groupe des Infusoires, nous constatons l'isogamie et l'anisogamie à divers degrés. Presque tous les groupes d'êtres inférieurs nous montreraient des variations analogues.

Sans quitter encore le cas des Infusoires, demandons-nous dans quelle mesure la conjugaison, arec la fusion des noyaux (caryogamie) qui en est le trai 
essentiel, est une nécessité pour la perpétuité de ces êtres. Au moment où Maupas effectuait ses recherches (1889), Weismann renait d'opposer les Protozoaires aux Métazoaires. Les premiers lui semblaient capables de se diviser indéfiniment; ils étaient immortels, alors que la vie de chaque Métazoaire est fatalement limitée. Le corps, ou soma, ne peut échapper à la mort ${ }^{1}$; le germen seul est immortel; il traverse le temps dans une série d'enveloppes somatiques successives. Chez les Protozoaires, soma et germen ne sont pas distincts. La totalité de l'être se continue indéfiniment; en d'autres termes, les Protozoaires seraient immortełs par essence.

Travaux récents modifiant la théorie de Maupas. Maupas trouvait, dans la nécessité du rajeunissement caryogamique, telle qu'elle résultait de ses expériences, une réfutation des idées de Weismann. Ses recherches, outre leur intérèt propre, araient ainsi alors une portée philosophique considérable. Ses conclusions ne semblent cependant pas aujourd'hui exactes, d'une facon absolue, après les expériences nourelles de Calkins, Enriques, Woodruff. Enriques regarde la sénescence observée par Maupas, non comme un phénomène physiologique survenant régulièrement, après un nombre élevé de divisions, mais comme une manifestation pathologique, due à des intoxications par le milieu extérieur, dans les conditions des cultures. En se plaçant dans des conditions farorables, on éviterait, d'après lui, cette sénescence. Calkins, de son côté, a bien constaté des phénomènes de sénescence, comme Maupas, mais il a pu les faire disparaître et rajeunir ses cultures,

1. La multiplication pratiquement indéfinie de certains Métazoaires, par voie asexuée ( $v$. chap XX, p. 26T) et de beaucoup de Végétaux est une exception à cette loi. 
à plusieurs reprises, sans conjugaison, en changeant simplement le milieu nutritif, où il faisait rivre, jusque-là, les Infusoires. Il réalisait là un phénomène analogue à la parthénogénèse expérimentale, puisqu'il suppléait à a fécondation par des actions d'ordre physico-chimique. Par ces mêmes procédés, Woodruff était arrivé, au début de cette année, à obtenir 3.300 générations successives de Paramécies, sans qu'il se soit interposé de conjugaison, alors que, dans les expériences de Maupas, lá zẻnescence survenait après 200 à 300 divisions consécutives.

Ainsi, le rajeunissement caryogamique n'apparait plus comme une nécessité physiologique absolue. Mais ces contradictions mettent surtout en évidence combien, mème sur des organismes aussi simples, aussi petits, aussi aisés à cultiver, il est difficile de réaliser des expériences véritablement comparables, où le déterminisme est le mème. Si cette difficulté est déjà énorme, quand on n'envisage que les conditions extérieures à l'organisme, elle l'est bien plus encore quand on remarque qu'il faut tenir compte aussi des propriétés intrinsèques de celui-ci, de son hérédité. Dans les Paramécies, - l'Infusoire sur lequel ont été faites toutes ces expériences, - il y aurait dans la nature, suivant Jennings, de nombreuses races, avec des propriétés héréditaires différentes : les unes, chez lesquelles la conjugaison scrait une nécessité fréquente et impérieuse, d'autres, où elle serait beaucoup plus rare, et d'autres, tafin, qui pourraient se propager indéfiniment par division. Et cela est aussi plausible, pour ces Protozoaires, que les différences observées entre les races, ou espèces, des Daphnies, des Rotiferes ou des Pucerons, chez lesquels la parthénogénèse se perpétue pour les uns et ne peut durer pour les autres.

J'ai insisté sur le cas des Infusoires, un des groupe's 
qui ont donné lieu aux recherches les plus nombreuses et les plus importantes, pour montrer combien les problèmes de la sexualité sont complexes, même chez les organismes inférieurs.

§ 2. - Sexualité des autres Protozoaires, en particulier des parasites. - Pour être moins connus et moins fouillés, ils ne doirent pas être plus simples chez les autres Protozoaires. Aujourd'hui. l'existence de phénomènes sexuels, de gamètes se fusionnant pour former un zygote, est constatée avec une généralité de plus en plus grande dans toutes les classes de Protozoaires: Rhizopodes, Flagellés, Sporozoaires, etc. Dans presque tous les groupes, on roit toute la gamme de la différenciation des gamètes, depuis l'isogamie parfaite jusqu'à l'anisogamie la plus accentuée : le macrogamète, gros et immobile, a alors l'aspect d'un ovule et le microgamète petit, mobile et pourru de cils ou flagelles, a toute l'allure d'un spermatozoïde. Il ne peut-être question ici de multiplier les exemples à l'appui de cette affirmation. La figure 42 en montre quelques-uns, chez les Volvocinées (Flagellates), les Grégarines et les Coccidies (Sporozoaires).

Les conditions dans lesquelles se manifeste la sexualité chez ces organismes, et où elle se substitue à la multiplication par division, sont très complexes, très variables d'une forme à l'autre et très mal connues. On verra des Volvox, Flagellates coloniaux verts qui peuplent beaucoup de nos mares, se multiplier longternps par voix asexuée ou parthénogénétique, et brusquement apparaitront les gamètes et la conjugaison. Il y a certainement un lien entre cette apparition et les conditions extérieures, telles que la température, l'éraporation de l'eau et l'approche de la dessiccation dans des mares restreintes, etc., mais 
on ne peut, au moins actuellement, réduire ce rapport à une formule simple et générale. Il en est de tous ces cas, comme de celui des Infusoires.

Plus aisé à analyser est, au contraire, le cas de nombreux parasites. Ici, la multiplication asexuée et
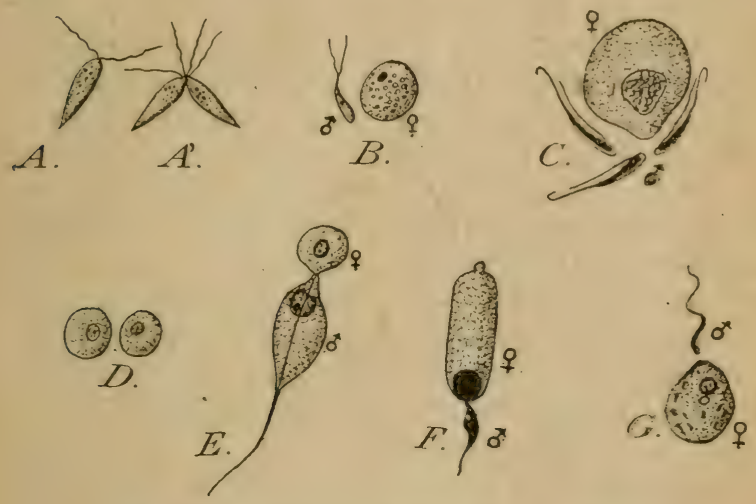

Frg. 42. - Exemples de Gamètes chez les " "otozoaires.

A. et $A^{\prime}$, gamètes et conjugaison (isogamie) de Stephr .sphæra (Volvocinée) (d'ap. Hieronymus); $B$, microgamète $\sigma^{7}$ et macroganète $O$ (anisogames) d'Eudorina (Volvocinée), (d'apr. Gcebel) ; C', microgamète ơ et macrogamètes (anisogames) d'une Coccidie (Eimeria Schubergi) (d'ap. Schaudinn); D, Gamètes (isograrnes) d'une Grégarine; $E, F$, Gamètes, ơ et $q$, anisogames d'autres Grégarines ( $E$, Stylorhynchus; $F$, Pterocephalus) (dap. Léger et Duboscq); $G$, Gamètes $\sigma^{*}$ et $\%$, anisogames, d'une Ilémosporidie (Hæmoproteus, d'ap. Schaudinn).

les phénomènes de sexualité se succèdent souvent en un cycle régulier, déterminé par les nécessités de la propagation du parasite. Il s'est fait une adaptation de la sexualité du parasite, aux conditions très spéciales de son existence : les Sporozoaires nous offriront des cas très typiques à cet égard et anj̧วurd'hui bien connus. 
Sporozoaires: Coccidies. - Prenons une Coccidie et choisissons une espèce (Coccidium oviforme), qui vit dans les cellules du foie du Lapin. Quand un Lapin ingère, les spores de ce parasite, les germes (sporozoïtes) qu'elles renferment sont mis en liberté dans l'estomac, pénètrent dans les celliales hépatiques et s'y multiplient arec une extrême rapidité, déterminant, pendant quelques jours, une infection aiguë. Pendant cette phase, il n'y a que multiplication asexuée, schizogonie, comme l'on dit. Au bout de quelques jours, l'organisme du Lapin, s'il ne succombe pas, réagit; les conditions du milieu sont changées pour le parasite, d'une façon qui n'a pas été exactement analysée jusqu'ici, et la Coccidie, au lieu de continuer à se propager asexuellement, par schizogonie et à envahir darantage son hôte, forme, d'une part, des gamètes mâles, en forme de spermatozoïdes; de l'autre, des gamètes femelles, qui sont fécondés par les premiers.

Le résultat de ces fécondations est la formation de kystes, dans lesquels se différencient les spores. Les formes schizogoniques, vivant dans l'intérieur des cellules, étaient nues; elles ne peuvent subsister en dehors de l'hòte. Kystes et spores, protégés par des membranes, peurent, au contraire, résister dans le milieu extérieur $e^{2}$ - passer ainsi passirement d'un Lapin à un autre. Ces kystes sont, en fait, éracués arec les excréments du Lapin et c'est par eux que le parasite se propage.

Ainsi, dans cet exemple, la sexualité apparait, à une phase déterminée d'un cycle érolutif, sous l'influence de modifications dans les conditions du milieu où vit le parasite dans son hôte, et, d'autre part, elle est liée à la production d'éléments de résistance, par lesquels le parasite pourra se transmettre à des hôtes nouveaux. 
Il en est ainsi chez un très grand nombre de parasites, Sporozoaires ou autres. L'apparition des gamètes est le prélude de la formation de spores résistant au milieu extérieur ou d'un changement d'hôte.

Hématozoaires. - Un exemple très significatif de ce fait nous est fourni par les parasit,s du sang et principalement des globules rouges des Vertébrés, auxquels on donne le nom d'Hématozoaires. L'un de ces organismes a, pour l'homme, une importance capitale, car c'est lui qui est l'agrent infectieux de la fièvre palustre ou paludisme, découvert, il y a trente ans, par Laveran. R. Ross a montré, en 1897, qu'il se transmettait d'homme à homme par l'intermédiaire de certains Moustiques, les Anophèles, et, dès lors, il a été possible aux zoologistes de reconstituer tout le cycle évolutif de ce parasite et des formes analogues. Or, on a constaté que, dans le sang de l'homme (ou de l'hóte rertébré pour les autres espèces), ces hématozoaires ne se reproduisent que par voie asexuée, par schizogonie; les périodes de multiplication schizogonique intense sont celles des accès fébriles. Jamais dans le sang, il ne se produit de processus de sexualité. Les gamètes se différencient, mais incomplètement; on observe, au contraire, immédiatement leur achèvement et parfois leur rencontre, dès que le sang est sorti du vaisseau et qu'ainsi les conditions do milieu sont changées pour le parasite. Dans la nature, c'est précisément ce qui arrive quand un Moustique suce le sang palustre. Aussitôt arrivés dans l'estomac du Moustique, les gamètes des Hématozoaires achèvent de se différencier, et la fécondation a lieu. Ici encore le processus sexuel est corrélatif de la transmission du parasite d'un hôte à l'autre, mais dans des conditions notablement différentes du cas des Coccidies.

25. 


\section{\$ 3. - Y a-t-il une méiose chez les Protozoaires?}

- On a recherché arec soin si les phénomènes de sexualité, qu'offrent les Protozoaires, comportaient des processus correspondant à la réduction chromatique et à l'expulsion des globules polaires. On a retrouvé, dans certains cas $^{1}$, des divisions nucléaires, préalables à la formation des noyaux des gamètes proprement dits, qui rappellent l'expulsion des globules polaires. Mais, d'une façon générale, on ne peut dire que l'on ait ici l'équivalent des divisions maturatives et de la méiose chromatique. Au reste, la division cellulaire, chez les Protozoaires, offre beaucoup moins d'uniformité que chez les Métazoaires. Il n'y a pas lieu d'entrer ici, à cet égard, dans des comparaisons détaillées : nous devons nous borner à enregistrer cette constatation négative.

§ 4. - L'autogamie. - Les Protozoaires ont, d'autre part, conduit à observer une catégorie particulière de processus nucléaires se rattachant à la sexualité, mais ayant une physionomie spéciale, je veux parler de l'autogamie?

Dans la fécondation, les éléments qui se fusionnent, ovule et spermatozoïde, sont des cellules d'origines différentes, provenant, le plus généralement, d'individus distincts. Mème dans les cas d'autofécondation, les gamètes sont séparés de la cellule ancestrale commune, par une très longue chaîne de divisions intermédiaires. Chez de nombreux Protozoaires, de groupes très variés, on voit, dans des circonstances équivalant manifestement à la conjugaison ou à la fécondation, se fusionner deux noyaux ou deux cellules qui sont sœurs, - étant issues immédiatement

1. Conjugaison des Infusoires, de certains Héliozoaires (Actinophrys).

2. Et de ses variétés : pædogamie, etc. 
d'un même noyau ou d'une même cellule mère. C'est là l'autogamie proprement dite. D'autres fois les gamètes ont une parenté encore précise, mais moins proche; ils descendent, par un petit nombre de divisions cellulaires, d'un élément générateur commun.

Je ne puis non plus entrer, à cet égard, dans des précisions circonstanciées. L'examen approfondi des recherches récentes conduit d'ailleurs à supposer qu'on a sourent exagéré la place que tiennent ces phénomènes. Beaucoup de cas signalés sont douteux, mais d'autres sont indéniables.

Il est nécessaire de mentionner ici leur existence, parce qu'ils apportent une restriction à la conception courante, d'après laquelle la fusion des éléments nucléaires, dans la fécondation, ne peut avoir lieu, d'une façon absolue, qu'entre cellules appartenant à des lignées tout à fait indépendantes. C'est la vérité générale, surtout chez les organismes supérieurs; mais, chez les êtres inférieurs, cela parait ètre beaucoup moins nécessaire, et l'autogamie représente la condition tout à fait opposée. 


\section{CHAPITRE XX́III}

\section{LA SEXUALITÉ CHEZ LES THALLOPHYTES}

Pieproduction asexuée : spores, zoospores. - Reproduction sexuée par gamètes. - Diversité des gamètes. - Cas des Jiatomées, Desmidiées, Levures, etc. - Gamètes mobiles Flagellés: - Isogamie et degrés divers d'anisogamie. - Characees, Fucus, Volvocinées. - Gamétangie des Mucorinées et de divers autres Champignons.

Sexualité purement physiologique de divers Champignon et Algues. - Mucorinées homothalliques et hétẻrothalliques.

L'autogamie chez les Thallophytes.

La sexualité, chez les Champignons et les Algues. se présente, comme chez les Protozoaires, dans des conditions et avec des aspects morphologiques extrêmement rariés et qui nécessiteraient de longs développements. Il en est de même si on l'envisage physiologiquement. Force nous sera donc de laisser de côté tout détail et de nous borner à quelques aperçus généraux ${ }^{1}$.

Chez tous ces êtres, l'individualité est très mal définie, et nous ne derons pas nous étonner de roir la reproduction asexuée et la reproduction sexuée se

1. Je renvoie pour une étude détaillée de ces questions aux ouvrages de Vuillemin et de Guilliermond sur les Champignons. On pourra lire aussi un intéressant article d'ensemble de Pavillard. (Revue Scientifique, du 8 mars 1913.) 
pénétrer intimement. La premiẻre mème est très difficile à délimiter du fractionnement plus ou moins accidentel et irrégulier de l'appareil végétatif.

Spores et gamètes. - Nous n'approfondirons pas ici ces distinctions, et nous nous bornerons à considérer les cas, où on est en présence d'éléments repro-
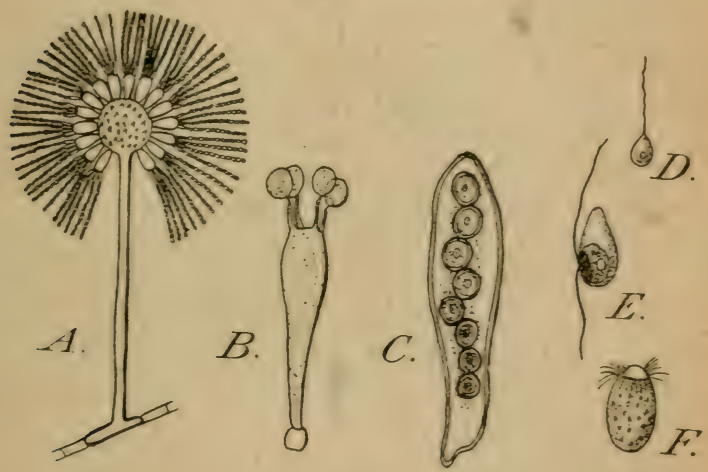

Fig. 43. - Divers̀ exemples de spores chez les Thallophytes.

$\boldsymbol{A}$, Conidiophore et conidies en chapelets de Sterigmatocystis niyra (d'ap. Van Tieghem!; $B$, une baside avec ses quatre spores; $C$, une asque avec ses huit spores.

Flagellispores (ou Zoospores) : $D$, de Chytridinée (Olpidium); $E$, d'Algue (Chorda filum dap. Reinke); $F$, d'Edogonium (d'ap. Pringsheim).

ducteurs différenciés. Suivant que ces corp: se fusionnent deux à deux ou non, il s'agira de reproduction sexuée ou asexuée, de gamètés ou de spores.

Quelques mots d'abords sur ces dernières, dont les Champignons et les Algues nous offrent une grande variété.

Chez les Champignons, les spores sont généralement immobiles; ce sont de petits corps, le plus souvent 
sphériques. Elles naissent souvent en grandes quantités, en bouquets ou en files, sur des filaments pl s ou moins particuliers du thalle, comme le montre la figure $43 \mathrm{~A}$ et on les désigne généralement souss le nom de conidies. Il s'en forme aussi dans dt's appareils plus spécialisés, que l'on appelle des basid, s ou des asques.

Chez quelques groupes, les spores sont mobiles à l'aide d'un flagelle; elles prennent alors le nom de zoospores. Tel est le cas, notamment, chez les Chrtridinées, Champignons inférieurs parasites.

Les éléments reproducteurs asexués des Algues sont toujours des zoospores, qui ont la structure d'un petit Flagrellé, muni d'un ou plusieurs flagelles; elles offrent parfois des différenciations assez grandes dans leur cytoplasme. Elles sont produites généralement en nombres énormes. Au bord de la mer, à marée basse, les flaques tapissées d'Algues, telles que les Ulves, sont parfois vertes, tellement elles renferment de zoospores.

Entre ces éléments asexués et des gamètes, la distinction est souvent très difficile.

La diversité des gamètes est énorme. Chez les types les plus simples, les Algues unicellulaires, comme les Diatomées (fig. $44 B$ ) et les Desmidiées ou les Levures parmi les Champignons, c'est l'individu tout entier qui, à un moment donné, constitue un gamète. Ces organismes, en effet, se multiplient, par simple division, répétée un grand nombre de fris, en deux individus indépendants, comme chez les Infusoires. Puis, à un moment donné, deux de ces individus, généralement d'origines différentes, s'accolent, et fusionnent leurs cytoplasmes et leurs noyaux, suivant des processus complexes, que nous n'étudierons pas en détail. Il y a ainsi production d'un zygote, qui se divise ensuite en deux individus de même forme 
que les parents et qui se séparent. Les deux gamètes qui se sónt associés sont tout à fait semblables; il y a parfaite isogamie.

La sexualité se présente dans des conditions également très sommaires, chez certaines Algues, formant

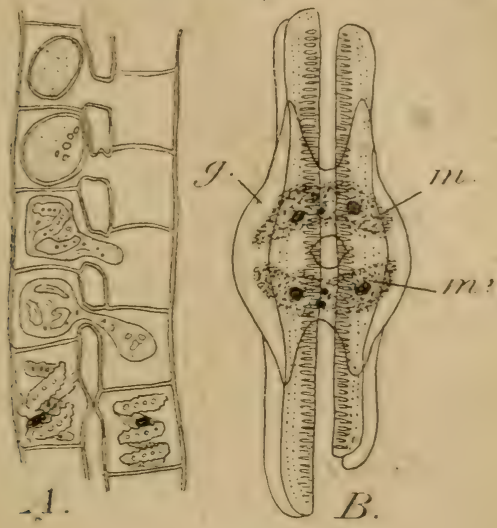

Fig. 44. - Conjugaison d'Algues.

1. Conjugaisons multiples, cellule ì cellule, entre deux filaments paralleles de Spirogyres; de bas en haut, stades suecessifs de la formation de l'ceut (dap. Schenck); $B$, conjugaison de deur Diatomées (Rhopalotia, dap. Kilebahn): les deux Diatomées sont côte à còte; les masses de cỵtoplasine ont fait saillie au dehors et ont donné nuissance à deux masses mixtes nouve!les $m$ et $m^{\prime}$, ou se font des fusions nucléaires et qui deviendront deus Diatomées nouvelles; $g$, enveloppe gélatineuse.

de simples filaments, constitués chacun par une file unique de cellules. Telles sont les Conjuguées (Spirogyres, etc.). Deux filaments (fig. $44 \mathrm{~A}$ ), disposés parallèlement, saccolent et il se fait une fusion des cellules placées en regard; tout le long des deux filaments, les protoplasmes se confondent en une masse, qui est l'œuf, ou zygote, et qui est mis en liberté ultérieurement.

Dans la plupart des Algues, les gamètes sont do 
petites cellules, mobiles à l'aide de flagelles et qui ne se distinguent parfois des zoospores que par une différence de taille. En présence de ces éléments, il est souvent très difficile de se prononcer sur leur rỏle véritable, d'éléments asexués ou sexués. Chez certains types, d'ailleurs, ils sont, suivant les circonstances, l'un ou l'autre. C'est le cas, par exemple, dans les algues du genre $\boldsymbol{P}$ rotosiphon.

On peut voir, dans des faits de cet ordre, la sexualité à l'état d'ébauche. Les gamètes peurent être considérés comme dérivant de zoospores. Dans beaucoup d'Algues, les deux catégories zoospores et gamètes, coexistent. La distinction entre elles se complique encore du fait qu'il y a parfois des zoospores de deux tailles différentes, qu'on serait tentě de considérer comme des gamètes mâles et femelles. Cela se présente dans les algues du genre Ulothrix; il y a des macrozoospores et des microzoospores; elles diffèrent par la taille, mais elles ont, les unes et les autres, quatre flagelles. Les gamètes, qui sont sensiblement égaux entre eux, ne sont au contraire pourvus que de deux flagelles (fig. 45, $A$ ). Ils peuvent d'ailleurs se développer parthénogénétiquement.

Isogamie et anisogamie. - On trouve, parmi les gamètes proprement dits, suivant les types d'Algues, tous les cas possibles : soit l'égalité parfaite, l'isogamie, soit une inégalité ou anisogamie plus ou moins marquée, soit enfin une inégalité très forte rappelant tout à fait ce que montrent les végétaux supérieurs ou les animaux.

Ainsi, chez certaines algues, i. is abondantes dans nos eaux douces, les Chara, les phénomènes sexuels raprellent beaucoup ceux des végétaux plus élevés, qui constituent le groupe des Archégoniées. Les gamètes mâles (fig. $45, B$ ) ont la forme typique des 
anthérozoïdes de ces dernières plantes : les gamètes femelles, ou oogones, sont de grosses cellules immobiles, protégées par un appareil, qui n'est pas sans analogie avec l'archégone. La fécondation se présente comme chez les Archégoniées.

L'existence de la parthénogénèse, chez les Characées, achève de rapprocher la reproduction sexuée de ces Algues de celle des Végétaux plus élevés, comme les Archégoniées, et de celle des animaux. Il y a, en effet, une espèce, Chara crinita, dont on ne connait jusqu'à présent uniquement que des plantes femelles, dans la plupart des localités ${ }^{1}$.

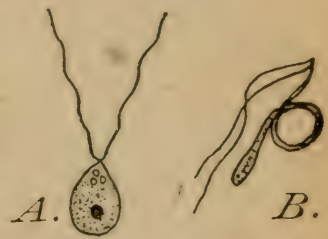

FN. 45. - Gamètes d'Algues : $A$, d'Ulothrix zonata (d'ap. Klebs); $B$, Anthérozolde de Sexualité des Fucus. - Les Characée(Niitella, d'ap. Sachs) Fucus, algues brunes qui recouvrent la plupart des rochers, dans la zone de balancemen't des marées, offrent un cxemple parfait et célèbre de reproduction sexuée, avec une anisogamie aussi complète que chez les animaux. C'est même, chez ces organismes, que la pénétration de l'élément mâle dans l'élément femelle, c'est-à-dire la fécondation, a été, pour la première fois, observée, en 1855, par Thuret.

Sur les frondes des Fucus, on distingue des poches sphériques, à orifice étroit, qu'on appelle des conceptacles. Dans certains d'entre eux, se différencient les gamètes femeiles ou oogones, rappelant tout à

1. En quelques localités cependant, on a trouvé des plantes mâles. Chara crinita se comporte donc, au point de vue de la parthénogénèse, comme Psyche helix chez les Lépidoptères, ou Apus chez les Crustacés. 
fait les oufs des animaux : ce sont des sphères opaques mesurant un diamètre d'environ

Dans d'autres conceptacles, se forment les gamètes mảles, véritables spermatozoïdes, minuscules, pourvus de flagrelles ${ }^{1}$. En mélangeant, dans une goutte d'eau de mer, les deux catégories d'éléments mûrs. Thuret a vu, sous le microscope, les spermatozoïdes s'accumuler autour des oogones (fig. 46), imprimer

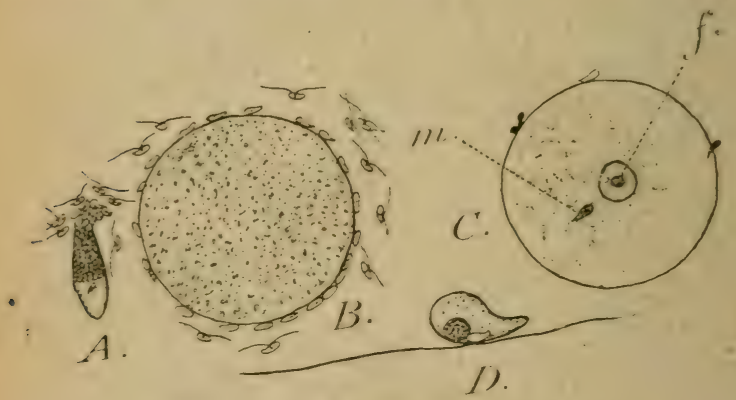

FiG. 46. - Gamètes et conjugaison des Fucus.

A, Anthéridie laissant échapper des anthérozoìdes; $B$, gamète femelle (oosphère) entouré d'anthérozoides qui le font tourner (d'ap. Thuret); $C$, oosphère fécondé ; $m$, noỵau mále, $f$, noyau femelle (d'ap. Farmer); $D$, anthérozorde plus grossi (d'ap. Guignard).

à ceux-ci un mouvement de rotation, jusqu'à ce qu'un de ces spermatozoïdes ait pénétré à l'intérieur. Ce n'est que vingt ans après Thuret, qu'on a pu observer les pł.énomènes parallèles de la técondation, chez les animaux, sur les Echinodermes.

Cas divers. - Dans un mème groupe d'Algues, on

1. Certains Fucus présentent, sur la meme fronde, les deux catégories de conceptacles: ils sont hermaphrodites (ex.: Fucus platycarpus); d'austres ont des frondes unisexuées, mâles ow femelles (ex. : Fucus vesiculosus, Fucus serratus). 
trouve parfois toute la gamme des cas allant de l'isogamie à l'anisogamie. Je me bornerai à citer un exemple qui, d'ailleurs, est revendiqué également par les zoologistes, celui des Volrocinées. Ce sont des êtres flaggellés unicellulaires, mais vivant associés en colonies plus ou moins nombreuses et parfaitement mobiles. On les range, tantôt dans les Protozoaires Flagellates, tantôt parmi les Algues rertes, arec qui elles ont en commun de posséder de la chlorophylle. Les Volrocinées se multiplient asexuellement ou par samètes. Partant des Stephanosphrera, où les gamètes sont parfaitement égaux, on trouve une série de degrés d'anisogamie qui conduisent jusqu'aux Eudorines et aux Volvox où les éléments mâles et femelles différent l'un de l'autre, comme ceux des Fucus ou comme les œufs et les spermatozoïdes des animaux.

Les Champignons, à leur tour, nous offriraient quelques exemples d'anisogamie comparables aux précédents. Je me borne à mettre ici, sous les yeux, celui des Monoblépharidées (fig. 48, A, p. 308). Chez eux, le même filament produit : à son sommet, un gamète femelle sphérique et, au-dessous, des microgamètes, munis d'un flagellum unique, qui s'échappent et viennent ramper à la surface de l'élément femelle jusqu'à ce que l'un d'eux y pénètre.

Tous les cas précédents rappelaient plus ou moins directement ce que les animaux, Protozoaires ou Métazoaires et les régétaux supérienrs (surtout les Archégoniées) nous avaient appris. Mais à cela ne se borne pas la rariété d'aspect et de constitution des éléments sexuels chez les Thallophytes. Dans le grand groupe des Algues rouges ou Floridées, la sexualité et les gamètes ont une allure toute particulière, que je me borne à signaler ici d'un not, ne pouvant développer suffisamment ce sujet.

La sexualité d:s Mucorinées. Gamétanges. - Chez les 
Champignons, on connaît aujourd'hui des phé mènes de sexualité dans presque tous les groupes; mais la nature et la forme des gamètes est très variée. Ne pouvant ici examiner tous les cas, j'en indiquerai seulement un, pris dans des formes très vulgaires, les moisissures ou Mucorinées. Comme tout le monde sait, ils forment une sorte de feutrage de filaments, le mycélium, sur lequel, de place en place, se forment des appareils sporifères. Par ces spores, extrêmement nombreuses, les moisissures se propagent et se sèment spontanément partout. C'est leur mode de reproduc-
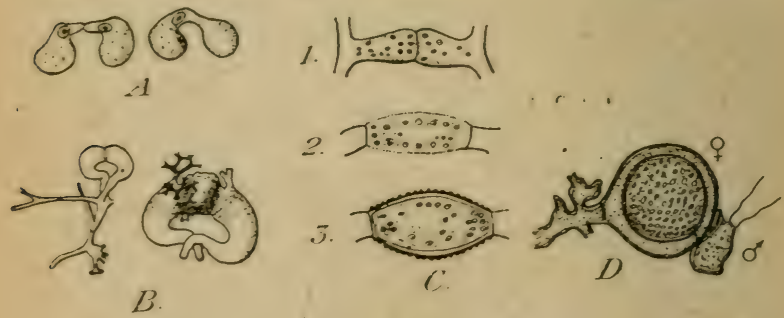

Fig. 47. - Conjugaison chez les Champignons.

A, 2 stades de la conjugaison chez une Levure (d'ap. Guilliermond); $B, 2$ stades de la formation de l'cuf (fusion de deux filaments) chez une Mucorinée (Phycomyces, dap. Yan Tieģhem); $C, 1-3$, mème phénomène; figure montrant que les filaments yui se fusionnent renferment chiacun plusieurs novaux (gamélanges; dap. (iviliiermond); $D$, conjugraison anisogame d'une Péronosporée (Cystopus candidus); $C^{*}$, anthérozoide; $\%$, oosphère. (D'up. de Bary.)

tion asexuée. Mais, en outre, à certains moments, dans des conditions qui ne sont pas toujours bien précisées, deux filaments roisins enroient, à la rencontre l'un de l'autre, deux diverticules, qui s'affrontent et se fusionnent. Puis une cloison isole la portion fusionnée, qui se couvre d'une membrane épaisse et constitue un ceuf ou zygospore (fig. 47, B). Cet œuf passe à l'état de vie ralentie et germera ultérieurement. co 
Telle est l'allure de la sexualité chez ces Champignons. Non seulement la forme des gamètes y est très particulière, - ce qui s'explique en partie parce que les phénomènes se passent à l'air, c'est-à-dire à sec, - mais, tandis que, dans la généralité des cas envisagés jusqu'ici, chaque gamète arait la valeur d'une cellule et que l'œuf était la fusion de deux cellules (son noyau provenant de la fusion de deux noyaux gamétiques), ici, les deux éléments qui se fusionnent renferment chacun un grand nombre de noyaux, et, dans l'œuf, il se fait toute une série de fusions, nucléaires. On peut donc interpréter ces éléments comme correspondant chacun à une pluralité de gamètes ébauchés dans une enreloppe commune. C'est ce qu'on exprime en disant que ce sont, non des gamètes, mais des gamétanges (fig. $47, C$ ) et le processus lui-même porte le nom de gamétangie.

La gamétangie se présente arec des variations étendues, dans les Mucorinées, les Saprolégniées, les Chytridinées, chez certains Ascomycètes, etc.

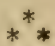

Nous avons considéré jusqu'ici la sexualité des Thallophytes, au seul point de rue de la forme et de la constitution des gamètes; mais cela n'est pas suffisant pour la connaitre complètement. Il y aurait, en effet, tout d'abord, à rechercher quelles conditions déterminent, chez ces régétaux inférieurs, l'apparition des processus sexués; car la plupart se propagent plus ou moins longtemps par voie asexuée et la formation des gamètes est liée à des circonstances plus ou moins définies. Il n'est pas possible de donner à cet égard des indications générales; il faudrait faire une étude détaillée d'une série de cas particuliers.

Mais il est intéressant d'examiner brièrement un 
autre aspect physiologique de la sexualité chez ces organismes, je veux dire les affinité des gamètes, l'un pour l'autre, dans les différents cas.

Quand il y a une forte anisogamie, il y a toujours attraction et fusion féconde des deux gamètes. quelles que soient leurs provenances (et elles sont en général différentes). Il n'en est pas de même quand les gamètes sont semblables.

Dans le cas d'une isogamie plus ou moins parfaite, il n'y a pas de différence de structure visible entre les deux éléments qui se conjuguent. Mais deux de ces éléments frères, issus d'une même plante productrice ou mieux encore d'une même cellule productrice, se conjuguent-ils entre eux ou doivent-ils, pour cela, provenir de pieds différents de la plante? On connait un certain nombre de cas, chez les Algues, où lá conjugaison ne peut avoir lieu qu'entre gamètes nés sur des plantes distinctes. Je citerai aussi certaines espèces d'Ectocarpus (algues brunes), de Dasycladus (algues vertes), d'Ulothrix (algues vertes). Ainsi, bien que, dans ces espèces, tous les gamètes soient semblables, il y a, entre eux, des différences physiologiques, deux polarités correspondant aux deux sexes.

Par les mêmes considérations, on a eu l'explication de bizarreries que présentait la formation des œufs de certaines Mucorinées, chez les Champignons. Il $\mathrm{y}$ arait des espèces où on l'obtenait très facilement et d'autres, au contraire, où elle ne se réalisait qu'irrégulièrement. L'explication en a été fournie par les travaux de Blackeslee. Chez certaines espèces, telles que Sporodinia grandis, les deux gamétanges, qui se fusionnent, peuvent être produits par deux thalles quelconques; les conditions de leur formation peuvent donc toujours être réalisées dans une culture et on observe aisément la production des œufs. Au contraire, dans d'autres espèces, telles que 
Rhizopus nigricans, Phycomyces nitens, il existe deux catégories de thalles, que l'on n'a pas réussi à distinguer morphologiquement, mais qui sont physiologiquement différentes. Blackeslee les désigne par les symboles + et -. Il ne se formera d'œufs que si un thalle + est en contact, dans une culture, arec un thalle - ; il ne s'en formera jamais, au contraire, si la culture ne renferme que des thalles de même signe ${ }^{1}$. Le thalle issu d'une spore donnée a donc ici une véritable sexualité. Il semble même que, chez certaines espèces, les diverses spores d'un même sporange donnent toutes des thalles de même signe et que, chez elles, la formation des œufs n'a lieu que dans des cultures renfermant des thalles issus de spores, appartenant à des sporanges différents. On roit donc que la sexualité, chez les organismes inférieurs, peut ètre physiologiquement très marquée, sans qu'elle se traduise par des caractères morphologiques recon-

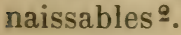

Autogamie. - A cette série de faits, s'opposent ceux d'autogamie, que l'on rencontre chez les Thallophytes, comme nous les avons déjà rus chez les Protozoaires. Ils consistent, comme on se le rappelle $^{3}$, dans la fusion de cellules jouant le rôle de gamètes et dérivant d'une même cellule-mère, soit

1. Blackeslee a appelé homothalliques les Mucorinées telles que Sporidinia et hétérothalliques celles qui ont deux catégories de thalles + et - .

2. Pinoy a constaté, chez les Myxomycètes, des phénomènes du meme ordre que ceux que nous venons de résumer chez les Mucorinées. Chez certaines espèces, telles que Didymium nigricans, les fructifications ne se produisent que si les plasmodes, qui leur donnent naissance, renferment des myxamibes de deux catégories; on peut aussi les désigner par + et - et rien ne les distingue, au point de vue morphologique.

3. Cf. ch. XXII, p. 294. 
immédiatement (antogamie proprement dite), soit par l'intermédiaire d'un petit nombre de générations cellulaires (pædogamie).

On connait depuis longtemps ce phénomène chez les Conjuguées; la formation de l'œuf, ou zygote, peut aroir lieu, chez ces Algues filamenteuses, par fusion des prolongements issus de deux cellules voisines d'un

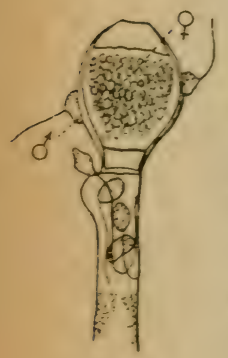

-1 .

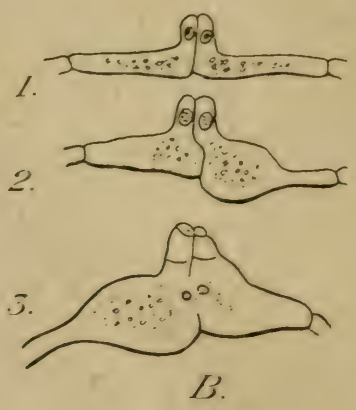

B.

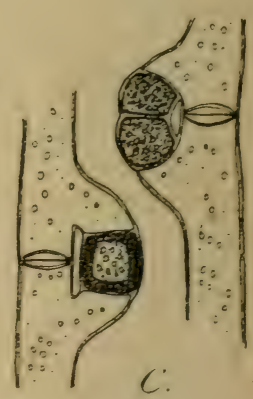

Fig. 48. - Exemples d'áutogamie :

A, conjuzaison dune Monołlépharidè : les anthérozoliles (O') et l'oosphère (Q) sont jroluits sur le méme filament (d'ap. Cornu); $B$, tusion de deux éléments consteutifs diun mime filament chez Basidiobolus (d'ap. Guilliermond); $C$, fusion de deux éléments consécutifs sur un filament de Conjuguée (Mougeotia, d'ap. Wittrock).

même filament (fig. 48, C), c'est-à-dire de deux cellules-scurs. Chez les Champignons, on en a signalé actuellement beaucoup d'exemples. Je me bornerai ici, pour fixer les idées, au cas d'une Entomophthorée (groupe parasite des animaux), le Basidiobolus ranarum, où l'œuf résulte d'une fusion entre deux cellules contiguës d'un même filament (fig. $48, B$ ). 


\section{CHAPITRE XYIV}

\section{LES CONDITIONS GÉNÉRALES DE LA SEXUALITÉ DANS LES ORGANISMES INFÉRIEURS}

Y a-t-il chez eux des globules polaires et une réduction chromatique?

Généralité des phénomènes de sexualité cỉez les Protozoaires et les Thallophytes. - Leur diversité. - Il se sont différenciés, de façons indépendantes, dans les divers groupes.

Le lecteur qui cherche, dans les organismes inférieurs, un tableau de la sexualité aussi analogue que possible à celui qu'offrent les types les plus élevés, animaux ou végétaux, ne manque pas de se demander, où est l'équivalent de la formation des globules polaires et ce qu'il advient de la réduction du nombre des chromosomes, préalablement à la fécondation.

Les globules polaires n'existent pas chez les végétaux, ni supérieurs ni inférieurs. Au moins n'a-t-on pas pu les décourrir jusqu'ici. Ceux qu'on a signalés, en particulier chez les Algues, sont des plus discutables. D'ailleurs, ce qui a été dit plus haut des Archégoniées et des Phanérogames et de l'alternance chez eux, du sporophyte et du gamétophyte montre que la réduction chromatique n'est pas liée, chez les Végétaux, d'une façon primordiale, à la fécondation, mais bien à la formation du gamétophyte. Or, la for- 
mation des globules polaires, chez les animaux, peut ètre considérée comme empruntant ses caractères spéciaux aux conditions où se réalise la réduction chromatique, à l'extrème fin de l'ovogénèse, au moment où l'ovule est mûr et va être fécondé.

Reste à voir, en ce qui concerne les Vécrétaux, ce qu'il advient de la réduction chromatique chez les Thallophytes. Les faits commencent seulement à se coordonner, malgré l'ardeur avec laquelle on a, depuis quinze ans, cherché à trouver dans ces Végétaux inférieurs l'équivalent de ce que montrent les Archégonićes; je ne puis faire qu'une allusion à ce problème qui nécessiterait des développements longs et critiques.

La réduction chromatique n'a été connue, parmi les Algues, jusqu'à ces dernières années, que chez un petit nombre de types, comme les Fucus. On l'a cependant mise en éridence, dans les Algues rouges ou Floridées, chez lesquelles des recherches très récentes conduisent à envisager une alternance régulière d'un sporophyte et d'un gamétophyte parallèles à ceux des Archégoniées. Et l'on tient à généraliser cette notion d'ure façon peut-être un peu subtile pour l'ensemble des Algues. Mème effort est fait, pour les Champignons. Une partie de ceux-ci ont un thalle cloisonné 1 et chaque portion du thalle, isolée par des cloisons consécutives, renferme à certains stades un seul noyau, à certains autres deux : on regarde l'état binucléé des cellules du thalle, comme la forme spéciale que revêt, dans ces organismes, la phase diploïde correspondant aux sporophytes des Archégoniées; l'état où chaque segment est uninucléé représenterait la phase haplö̈de ou de gamétophyte. On a suivi la transformation du dernier en le premier, dans quelques

1. Urédinées, Ascomycètes, Basidiomycètes. 
formes. D'autre part, le retour à l'état haploïde se fait par une fusion des deux novaux en un seul et le phénomène a lieu, d'après Dangeard, lors de la formation de l'appareil sporifère principal, c'est-it-dire des téleutospores chez les Urédinées, des asques chez les Ascomycètes, des basides chez les Basidiomycètes. Cette fusion est suivie de deux dirisions cellulaires, qui rapuellent celles de la phase méiotique des Archéogniées, ou des animaux.

Il serait certainement exagéré de dire que ces interpríations ont, dès à présent, une portée absolument cénérale et une valeur définitive. Elles tendent, comme on le sont, à étendre, à tout le règne végétal, les notins de sporophyte et de gamétophyte, qui résume:it si bien la sexualité des plantes plus élevées en orquisation. L'avenir dira si cette synthèse, qui est encice à l'état d'élaboration, est l'expression générale de la vérité.

En.c. qui concerne les Protozoaires, on n'est pas encore rrivé à un tableau aussi cohérent. Les phénomènes du la division nucléaire montrent d'ailleurs chez eux un - liversité, qui ést une difficulté préalable. Dans quelques cas, notamment chez certains Infusoires, on a constalé une réduction numérique des chromosomes, précédant la conjugaison que nous arons sommairement décrite plus haut. Chez les Infusoires et chez les IIéliozoaires, les préludes de la conjugaison comporifnt d'ailleurs des divisions nucléaires qui rappellent curtainement à beaucoup d'égards, la formation des globules polaires. Ici, où nous ne pourons entrer dans l'exposé et la discussion des détails, nous devons uous contenter de remarquer qu'on n'a pas encore constaté, d'une manière générale, dans l'ensemble des Protozoaires, tout au moins sous une forme précise, l'équivalent des processus de réduction chromatique et de la formation des globules polaires 


\section{$*_{* *}^{* *}$}

Le moment est venu maintenant de considérer l'ensemble des phénomènes de la sexualité chez les organismes inférieurs, Protozoaires et Thallophytes.

Nous constaterons, en premier lieu, la généralitẻ de ces phénomènes: dans tous les groupes, même les plus inférieurs, une étude suffisamment approfondie les a mis en évidence, et nul doute que nous n'en découvrions encore, dans bien des cas, où ils sont, pour le moment, inconnus.

La sexualité n'est donc nullement l'apanage des groupes plus élevés. Chez ceux-ci, elle occupe une place bien déterminée dans le cycle vital, toutes les fois que l'inciividualité est bien définie. La sexualité intervient alors strictement, au moment de la reproduction, lors de la formation de l'œuf. Dans les cas où l'individualité s'affaiblit, ce rapport cesse d'ètre aussi précis et l'on voit apparaitre une multiplication asexuée, qui peut, comme nous l'arons constaté, refouler plus ou moins les phénomènes sexuels, jusqu'à les faire même pratiquement disparaître.

Il n'est pas surprenant que, chez les Protozoaires et les Thallophytes, où, d'une manière générale, l'individualité n'est pas définie, la sexualité ait une place indécise dans le cycle. Elle n'est pas, à proprement parler, un processus de reproduction. La formation d'individus nouveaux, la multiplication, est asexuée et la sexualité n'intervient qu'à des intervalles plus ou moins réguliers et éloignés; tout se passe comme si elle apportait un correctif physiologique, plus ou moins indispensable, après un nombre élevé de reproductions.

C'est la conception que Maupas a formulée, sous le nom de rajeunissement caryogamique, par lequel il 
définit le rôle de la conjugaison chez les Infusoires. Mais nous sarons aujourd'hui qu'il ne faut pas prendre cette interprétation dans un sens trop absolu. Elle reste cependant rraie, dans la généralité des cas et le fait qu'une différenciation sexuelle physiologique rigoureuse existe chez des types inférieurs comme des Algues (Ulothrix, Ectocarpus, etc...), ou des Champignons (Mucorinées), ou des Myxomycètes, sans qu'elle s'accompagne de différenciation morphelogique, est en faveur de la conception de Maupas.

Au point de vue morphologique, alors que, chez les organismes plus élevés (Archégoniées, Phanérogames, Métazoaires), la sexualité se manifeste par une anisogamie très marquée et une division du travail entre les deux gamètes, chez les Protozoaires et les Thallophytes, nous voyons une extrème variété de cas et cela dans tous les groupes. Dans un grand nombre d'entre eux (Coccidies, Hématozoaires, Volvocinées, Fucacées, etc., etc...), est réalisée une différenciation anisogamique aussi complète que chez les Métazoaires. Nous nè pouvons donc pas dire que l'anisogamie soit le propre de ces derniers.

En somme, la sexualité se montre, chez les Protozoaires et Thallophytes, aussi complexe, sinon plus, que chez les Métazoaires. Sans doute, elle revèt chez certains d'entre eux des formes très simples; nous pourons les interpréter comme étant l'ébauche des processus que présentent les organismes plus élerés; mais il serait téméraire d'interpréter ainsi tous les cas de cet ordre. Il semble bien que pas mal d'entre eux soient des exemples, non de simplicité primitive, mais. de régression secondaire.

Les organismes inférieurs sont donc un vaste champ, d'un intérèt très considérable au point de vue des problèmes qui nous ont occupés ici, en raison surtout de la variété qu'ils nous montrent et qui s'oppose 
à I miformité des types supérieurs. Mais nous ne devons pas nous représenter que, dans l'ensemble, la sexualité y soit à l'état d'ébauche, et qu'elle ne se soit différenciée qu'à un niveau plus élevé de la complication organique. La différenciation s'est produite dans ces formes inférieures mêmes, autant, sinon plus, que chez les Métazoaires, et dans de multiples directions indépendantes. La sexualité s'est diversifiée et perfectionnée, dans chaque groupe de Protistes indépendamment. ou, si l'on veut, d'une façon polyphỵlétique. Il s'y est cependant conservé, à côté de processus tries spécialisés, des formes simples qui peuvent nous donner l'idée des phénomènes initiaux.

A ce point de vue comme aux autres, en dépit de la simplicité que leur confère leur structure unicellulaire, ou la faible différenciation relative de leur appareil régétatif, ces ètres sont, tout comme les plus cilevés, des organismes très vieux. Le passé pèse aussi lourdement sur les uns que sur les autres, quelle que soit l'empreinte qu'il a laissẻe sur eux.

Et, dans ce monde vieilli, les origines sont aussi difficiles à décourrir et aussi lointaines, quel que soit le niveau où on cherche à les déchitfrer. 


\section{CONCLUSIONS}

\section{GHAPITRE XXV}

\section{VUES GÉNÉRALES SUR LES PROBLĖMES DE LA SEXUALITÉ}

Dans quelle mesure la sexualité est-elle liée à la reproduction - Ln dernier mot sur les rapports de l'hermaphrodisme et du gonochorisme. - Conclusions relatives à la détermination du sexe. - La sexualité et l'ensemble de l'organisme. - Le sexe se détermine-t-il dans le soma ou dans le germen?

Arrivés au terme de ce livre, il convient, en guise de conclusion, de jeter un coup d'œil en arrière et d'en dégager les résultats principaux, comme les grandes masses qui, d'un sommet, font le caractère d'un paysage. Ce n'est pas qu'on ait envisagé ici la sexualitẻ sous tous ses aspects possibles. On a cherché à l'étudier dans son substratum organique et on a laissé complètement de côté tous les problèmes d'ordre psycholugique. Ils ont certes leur très grande importance, mais ils peurent ètre considérés comme une partie distincte du sujet, vis-à-vis de laquelle la matière de ce livre peut jouer le rôle des fondations souterraines d'un édifice. La psychologie de la sexualité est conditionnée, en effet, par les données d'ordres anatomique et physiologique. 
\$1. - Sexualité et reproduction, - Chez les animaux supérieurs, la sexualité est intimement liée à la reproduction; elle en est la condition préalable nécessaire. Cette conception est courante; elle est devenue presque instinctive en nous et elle garde, en fait, toute sa valeur. Il convient cependant d'y apporter une restriction théorique, si on la formule d'une façon générale. Chez les organismes inférieurs, surtout chez les unicellularres, la sexualité n'a nullement ce rapport nécessaire avec la reproduction. La multiplication se fait en dehors de toute sexualité et celle-ci n'intervient, dans la vie de l'espèce, qu'à intervalles plus ou moins rares et irréguliers, comme un correctif physiologique.

Chez divers Protozoaires, l'acte sexuel, la conjugaison, n'est nullement suivi de multiplication. La fusion de deux gamètes, dont le passé est différent, peut être conçue comme rétablissant une constance et un équilibre physico-chimique qui, dans une lignée asexuée, pouvaient tendre à être rompus, par l'action continue et prolongée de conditions spéciales. Il ne faut pas cependant voir là une notion absolue. L'irrégularité même avec laquelle se manifeste la sexualité, chez les Protozoaires libres, dans les cas où elle a pu être assez bien étudiée, l'indique suffisamment et c'est, en particulier, l'impression qui se dégage, pour quiconque étudiera les travaux de Maupas, Calkins, Enriques, Jennings, Woodruff, etc..., sur la sexualité des Paramécies, parmi les Infusoires.

Ainsi envisagée, la sexualité peut exister indépendamment de toute complication anatomique et de toute différenciation morphologique. Des individus d'apparences identiques différeront par la seule sexualité. C'est ce qu'enseignent les faits établis par Blackeslee sur les Mucorinées, par Pinoy sur les Myxomycètes, etc... Et, autant qu'on peut émettre des 
affirmations sur des phénomènes aussi complexes et aussi généraux, c'est là, sans doute, la forme et le rôle primitif de la sexualité dans les organismes.

La localisation de la sexualité, ou mieux la condensation de ses propriétés dans des gamètes, est vraisemblablement déjà un processus secondaire.

Il est aussi des plus plausibles que ces gamètes ont d'abord été semblables. Ensuite, s'est établie progressivement, entre eux, la division du travail, qui aboutit à l'œuf et au spermatozoïde, au macrogamète chargé des réserves et immobile d'une part, au microgamète mobile et dépourvu de réserves de l'autre. L'isogamie primordiale a conduit progressivement à l'anisogamie.

Cette dernière condition, constante et complète chez les Métazoaires et les Végétaux autres que les Thallophytes, peut ètre considérée comme ayant été réalisée antérieurement à la différenciation de ces organismes. Mais, quand s'est accompli le passage des êtres inférieurs, unicellulaires ou à thalles peu ou point différenciés, aux types plus élerés, Métazoaires et Archégoniées, il s'est fait, de par la complication organique même, une séparation plus tranchée et d'un caractère permanent, entre l'appareil végétatif et les éléments auxquels est restée dérolue la sexualité fonctionnelle, dans cette condition nouvelle. Le soma s'est séparé du germen. Cette séparation est déjà, soit ébauchée, soit même réalisée, chez certains des Protozoaires actuels; elle est à l'état d'ébauche chez les Infusoires, avec la dualité du macronucleus et du micronucleus; elle est beaucoup plus complète chez des formes comme les Volvox.

Les Métazoaires ont pu continuer à se reproduire par dirision ou multiplication de l'appareil régétatif; c'est ce que nous royons encere actuellement dans les phénomènes de reproduction asexuée que nous avons précédemment étudiés (Chap. XX). Mais ce mode 
de reproduction a dû vraisemblablement disparaitre assez rapidement, avec les progrès de la différenciation des organes, de la division du travail entre eux, de la solidarité entre les parties dans le tout, en un mot, arec les progrès de l'individualité. Nous le rencontrons encore là, où, pour des raisons surtout éthologiques, l'individualité est affaiblie, et, à ce titre, principalement chez les plantes. En ce qui concerne les animaux, j'ai dit que j'incline à considérer les divers cas de multiplication asexuée que nous' observons, comme ayant réapparu secondairement et non comme la persistance directe, jusqu'à nous, d'un mode de multiplication primitif.

La multiplication asexuée primitive ayant ainsi disparu, le mode de propagation qui a subsisté est celui où intervenait accessoirement la sexualité. La reproduction par gamètes est devenue la règle, et la sexualité s'est ainsi confondue en fait avec la reproduction, au point que les deux notions se sont identifiées pour les animaux supérieurs. L'amphimixie, c'est-à-dire la formation d'un œuf fécondé, est donc apparue d'abord, dans la biologie, comme le point de départ nécessaire et fatal du développement de tout être vivant;'d'où l'aphorisme: Omne vivum ex ovo, qui reste vrai, en fait, pour la généralité des animaux, mais comporte cependant une part de restriction résultant des considérations précédentes.

D'autre part, la parthénogénèse naturelle est venue montrer des exceptions à la nécessité de la fécondation; la parthénogénèse expérimentale nous autorise aujourd'hui à penser, d'une façon générale, que, théoriquement, la fécondation n'est pas une nécessité pour l'évolution de l'ovule. La reproduction apparait ainsi, de nouveau, comme dégagée, au point de vue théorique, de la fusion de deux gamètes. Toutefois, l'ovule parthénogénétique reste un gamète 
femelle, au point de vue morphologique, par son histoire antérieure et par sa constitution.

J'insiste bien sur ce que ces restrictions sont d'ordre théorique. En fait, la fécondation, et mème la fécondation croisée est la loi, et la fusion de deux samètes, ou amphimixie, a une importance capitale pour la vie et la stabilité de l'espèce; elle domine toute l'hérédité.

§2. - Hermaphrodisme et gonochorisme. - Nous pourons, en ce moment, à la lumière des faits acquis, confronter encore brièvement les deux états de la sexualité, l'hermaphrodisme, c'est-à-dire la réunion des deux types de gamètes sur un mème indiridu, et le gonochorisme, c'est-à-dire leur séparation sur des individus distincts. Quel est celui de ces deux états dont l'autre dérive? Dans la nature actuelle, nous avons dit que, suivant les cas, les deux alternatives possibles sont réalisées. On ne peut, d'autre part, trancher formellement le débat, en ce qui concerne les origines, mais simplement émettre des conjectures.

Sur ce terrain, la conception de la sexualité, qui vient d'être résumée, me parait devoir conduire à l'hypothèse que le gonochorisme est la condition primitive. Nous la voyons, en effet, réalisée, sans différenciation morphologique appréciable, chez les Mncorinées par exemple. L'amphimixie semble, d'après des faits de cette nature, aroir dû se produire, à l'origine, entre produits isolés d'individus différents, dont l'organisme entier avait, physiologiquement, l'un des deux sexes.

D'autre part, en dehors des cas d'autofécondation, - qui restent l'exception chez les animaux, - l'hermaphrodisme est encore un gonochorisme physiologique. Et les cas d'autofécondation, qu'on les envisage 
chez les plantes ou chez les animaux, apparaissent en corrélation arec une éthologie très spéciale, fixation, parasitisme, ete. L'ensemble des faits d'hermaphrodisme, dans la nature actuelle, est en faveur de l'hypothèse que c'est l'état secondaire et non l'état primitif, ainsi que nous l'avions conclu précédemment.

Son existence, soit comme phénomène général chez des groupes entiers, soit comme fait exceptionnel dans des espèces particulières ou des individus isolés, nous montre cependant qu'hermaphrodisme et gonochorisme ne sont pas des entités opposées et incompatibles. Dans aucun organisme, il n'y a d'impossibilité de principe à ce que s'élaborent, côte à côte, les deux types de gamètes. Mais, chez les espèces franchement gonochoriques, la production de l'un de ces types est, dans la règle, exclusive de celle de l'autre. Il en est ainsi, chez la généralité des animaux qu'on appelle supérieurs, et, parmi les problèmes de la sexualité, celui du déterminisme du sexe devient chez eux le principal.

\$ 3. - La sexualité et l'ensemble de l'organisme. - Nous arons vu ce qu'on pouvait actuellement conclure, au sujet de ce déterminisme. C'est en somme peu de chose et rien n'indique qu'il y ait une solution générale.

Les faits allégués jusqu'ici, en faveur de la détermination du sexe, au cours du déreloppement de l'embryon, sous l'influence de facteurs extérieurs, température, nutrition, etc - n'ont pas résisté à une critique serrée. C'est donc rers la détermination avant la fécondation, ou lors de celle-ci, qu'on se tourne. Le premier de ces cas se ramène en somme au second, au moins en général. Car, a priori, on doit admettre, il me semble, que l'élément capital du pro- 
blème est la composition physico-chimique définitive de l'œuf. Certes, elle peut être influencée par les conditions, où l'ovule a effectué sa croissance dans l'ovaire, c'est-à-dire par des circonstances d'ordre progamique - et nous avons relaté divers faits de cette nature -; mais la composition de l'œuf définitif n'est acquise que par la fécondation. Le spermatozoïde vient modifier très sensiblement l'état de l'ovule, antérieur à celle-ci. En définitive, c'est donc l'idée de la détermination syngamique qui est la plus logique, et c'est aussi à elle qu'actuellement les faits apportent l'appui le plus étendu.

Elle s'harmonise d'autre part arec les tendances les plus en faveur dans la biologie à l'heure présente, avec la conception mendélienne de l'hérédité, et arec d'assez nombreuses observations relatives aux chromosomes. Beaucoup de biologistes verront, dans ces deux ordres particuliers d'arguments, la solution mème du problème. J'ai indiqué les réserves qu'il y a lieu de faire, sur l'un et sur l'autre. Le mendélisme peut représenter assez bien les faits et être ainsi un moyen fécond d'investigation; mais il ne me parait pas devoir être considéré comme rérélant réritablement la réalité. Il n'est qu'un symbolisme. Et quant aux chromosomes, sans nier en aucune façon l'importance d'observations et de faits apportés par des hommes comme Boveri, Ed. Wilson et bien d'autres, je suis de la minorité qui se refuse,à y roir des individualités réelles, ni les porteurs spécifiques des propriétés héréditaires, de la sexualité comme des autres. Je crois donc qu'il ne faut pas exagérer actuellement la portée des conclusions qu'on a tirées de leur examen.

Ici, d'ailleurs, le problème de la sexualité en arrive à se confondre avec celui de l'hérédité, c'est-à-dire selui de la conception de l'organisme tout entier. 
Dewa poirss de vue se heartent actucllement sur ce ferrain : l'un, qui viacconda de reialite vécitabie qu'a Forganismo total et un; T'autre, qui subdivise cet creanisme en une mosalque indinie de propirieles dislinctes at indipendantes, de parties autonomes, et Sui chenche, d'une fagon plus ou moias simpliste et cousciente, ls représentation anticipte des unes et des autres, Cans des particules rastérielles da germe.

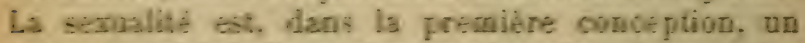
arpect special de l'organismon entier; dans la seconde, elle est Tune de ces inaombrables propridtes partielles, qui s'assemblent en un edifice et qui ont leur reprofentation dans tel ou tel chromosome an fraction de chromosome. Je me rallie nethement is is première de ces ounceptions.

Xais alors, si la serualite est ua aspect de lorgasistore encier, si clle limpritrne en quelque sorte, wa siege đ'est pas teulement dans les clandes génitales, nasis dans tous les or panes. Cest. oe que monIneut las caracteres semuels cecoondaines, que ton peut wolliplieo a l'iofini, par une observation suftisamment minutieuse, ef deceler dans toutes les parties te Porcanisme. On peut méme concevoir que la servalite soit plas ou moins definie, qu'elles soit peu marques, eu particulier, chez les espotces offreat un hemuaphrodisme exceptionnel on rudimentaire.

On doit ae demander surtout, ten se bormant aux organitmes gonochoriques, - et si l'on considere le asie comme resultant en difintive d'un etat phrsicochimigue de Tonganieme, - si clest Tembryon bout entier. qui, Afja proalablement a la diffenenciation Le da glande gewitale, reteatil sur trebanche de cetie Glands et Foriente wers un testioule ou vers an owaire. - wo Lien, an contraire. al cest la glande genitale. et avant elle les cellules génitales initicles, qui réagiscent, de par lenr constitution pbytion-chimique 
et le sexe particulier qui en découle, sur le rete de l'organisme, y manifestant les caractures sesuels secondaires corrélatifs du male ou de la famelle. Ie= germen determize-t-il la serualite dis soma, vu la sexualite du soma commande-t-alle la differennintiom du germen?

On saisit bien là, en tout cas, la penétration réciproque des problèmes relatifs au déterminisme du sexe et aux caracteres sexuels secundaires, problemes que nous avons envisagés separéraent dans deux parties successives de ce volume. Je ne trancherai pas l'alternative à laquelle nous venons de parvenir: peutEtre, au surplus, y a-t-il du vrai dans chacune des deux bypotheses, dont l'opposition serait jusgu' is un certain point artificielle?

Ces conceptions, enfin, permettent d'admettre que le sexe. dependant d'un état physico-ichimique de liauf et des premières cellules de lorganisme, puisse etre influence, dans certains cas, par les circoustances extérieures, au cours du développement. Les faits, je le répète, semblent montrer quil n'en fat pas ainsi; mais cela prouve seulement que l'equilibre serait, dès la fecondation, fortement rompia dans un sens ou dans l'autre. Si tel est le cas Général, il peut $y$ en axoir dautres, ou cette rupture d'équilibre soit moins accentuke, ou le fléau penche faiblement, sous l'influence de la constitution physico-chimique de l'ouf. c'est-átlire des facteurs internes, ou il penche assez peu, pour que la pression - si faible vis-a-vis de ceux-ci - que peuvent exercer les facteurs extérieurs, suflise cependant encore à définir le sens où il tombera definitivement. Clest dans les cas où la détermination du sexe aurait ainsi une labilité particulierement marquée, que les facteurs externes pourraient intervenir efficacement dans le résultat définitif. 
On trouvera peut-être ces conclusions bien éclectiques. Je n'aperçois pas qu'on puisse légitimement les rendre plus unilatérales.

$$
\text { ** }
$$

Si les solutions claires, simples et définitives, que nous affectionnons instinctivement, ne semblent pas particulièrement nombreuses dans ce livre et dans ses conclusions, au moins les considérations précédentes auront-elles dégagé, j'espère, l'unité globale et l'ampleur des problèmes de la sexualité.

Je souhaiterais que le lecteur y eutt pris conscience de ce que la Sexualité est un des aspects les plus généraux, sous lesquels nous apparait la vie, quand on en analyse les phénomènes, et qu'il eût senti les liens étroits, qui la rattachent aux questions essentielles de la physiologie cellulairés et de la biologie générale. 


\section{TABLE DES MATIÈRES}

AvaNT-Propos

\section{INTRODUCTION}

\section{LES CELLULES SEXUELLES OU GAMĖTES}

Chapitne I. - Les gamètes mûrs. . . . . . .

L'ovule. - Le spermatozoïde.

Chapttre II. - La genèse des gamètes. . . . . . .

Le tissu germinal. - Son origine chez l'embryon. Weismann et la distinction du soma et du germen. - Différenciation généralement très précoce du tissu germinal.

Ovogénèse. - Ovules et cellules folliculaires. Nutrition de l'ovule.

Spermatogénèse. - Spermatogonies et cellules nourricières. - Le spermatozoïde, comme l'ovule, est une cellule.

Chapitre III. - La maturation des gamètes et la fécon.

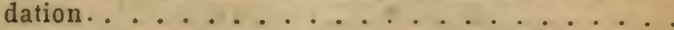

La phase de réduction chromatique terminant l'ovogénèse et la spermatogénèse. - Ovocyles et spermalocytes. - Réduction à moitié (méiose) du nombre des chromosomes.

La fusion des gamèles ou fécondation. - Pénétration d'un seul spermatozoüde dans l'orule (monospermic).

- La fécondation double, dans l'œuf, le nombre des 
chromosomes existant dans chacun des gamètes. Égalité d'apports paternel et maternel en chromatine.

Les chromosomes doivent-ils ètre considérés comme le support matériel et spécifque des propriétés héréditaires?

\section{PREMIÈRE PARTIE}

\section{L'HERMAPHRODISME OU RÉUNION DES SEXES}

Cuaprtre IV. - Les formes diverses de l'hermaphrodisme Autofécondation et fécondation croisée. - L'autofécondation chez les plantes et chez les animaux. Lignées pures de W. Johannsen. - Amphimixie.

Les formes diverses de lhermaphrodisme chez les animaux : structures diverses des glandes génitales. Hermaphrodisme simultané et hermaphrodisme successif (protandrie et protogynie). - Máles complémentaires.

Chapitre V. - L'extension et les degrés de l'herma-

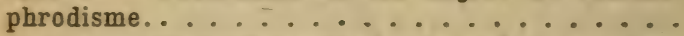

Hermaphrodisme normal; ses divers degrés de généralité. - Hermaphrodisme exceptionnel. - Recherches d'E. Maupas sur les Nématodes. - Hermaphrodisme rudimentaire : cas divers chez les Vertébrés (Myxine et autres Poissons; organe de Bidder des Crapauds, etc.). - Hermaphrodisme accidentel des Mammifères et de l'Homme.

L'hermaphrodisme est-il une condition primitive ou secondaire des organismes ?

\section{DEUXIÈJE PARTIE}

\section{LE GONOCHORISME OU SÉPARATION DES SEXES LES CARACTERES SEXUELS SECONDAIRES}

Cenpitre VI. - Dimorphisme et polymorphisme sexuels.

Caractères sexuels primaires et secondaires. - Degrés divers du dimorphisme sexuel. - Cas de dimorphisme 
sexuel intense; corrélations avec léthologie. - Diversité des caractères sexuels secondaires.

Polymorphisme sexuel : chez certains Papillons; chez les Insectes sociaux.

Gynandromorphisme.

Chapitre VII. - Les caractères sexuels secondaires et la reproduction. - La sélection sezuelle. . . . . Caractères sexuels secondaires en rapport direct avec la reproduction, ou aidant à la rencontre des sexes. Exagération du dimorphisme sexuel dans les périodes de reproduction.

Le dimorphisme sexuel peut-il s'expliquer par la sélection? - Théorie de la sélection sexuelle; sa crr tique.

Chapitre VIII. - Les caractères sexuels secondaires et lactivité fonctionnelle de l'organisme.......

Métabolisme dans les deux sexes et réalisation du type spécifique. - La castration nutriciale chez les Guêpes (P. Marchal) et les neutres des Insectes sociaux.

Chapitre IX. - Rapports entre les glandes génitales et les caractères sexuels secondaires. - Castration

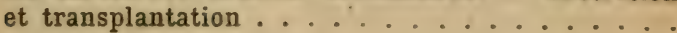

La castration par sénilité : arrhénoïdie et thélyidie. - Castration parasitaire. - Castration expérimentale; les eunuques.

La castration chez les Vertébrés altère les caractères sexuels sccondaires. - Résultats négatifs des expériences faites sur les Insectes.

La transplantation des glandes génitales : elle affaiblit ou annule les effets de la castration. - Expériences faites sur le Coq, sur la Grenouille. - Expériences de Steinach sur les Rats; inversion sexuelle.

Chapitre X. - Caractères sexuels secondaires et sécrétions internes (hormones)............

Les sécrétions internes ou hormones. - La fonction glycogénique du foie. - Généralité des actions hormoniques. - Appareils à sécrétions internes specialisées [(thyroïde, capsules surrénales). - Progrès récents :de nos connaissances sur ces actions. Action régulatrice générale des hormones dans lor- 
ganisme. - Coordination nerveuse et régulation chimique.

Lappareil génital agit sur iorganisme par des sécrétions internes. - Interfrétation des expériences sur la castration et la transplontation des glandes génitales. - Travaux de Bouin et Ancel. - Glande interstitielle du testicule. - Corps jaune de Tovaire. - Utérus et glande mammaire.

Valeur des différences entre les faits observes chez les Arthropodes et chez les Vertébrés.

\section{TROISIĖME PARTIE}

\section{LE PROBLĖME DU DÉTERMINISME DU SEXE CHEZ LES ANIMAUZ GONOCHORIQUES}

Canitra XI. - Les sexes et la méthode statistique . .

Lois statistiques des naissances tumaines. - Égalité numérique approximative des sexes chez la plupart des espèces. - Exceptions à cette règle.

Le principe d'autorégulation de Düsing. - Sa portée réelle. - Abus de la méthode statistique : elle ne nous permet aucune induction sur le mécanisme physiologique de la détermination du sexe.

Chartre XII. - L'époque de la détermination du seze.

Le sexe doit étre déterminé avant d'être effectivement reconnaissable. - Trois hypothèses générales possibles sur le moment de sa détermination: après, avant ou à la fécondation (épigamie, progamie ou syngamie).

I. - Hypothèse épigamique. - Expériences sur les Papillons, les Mouches, les Batraciens. - Conclusions négatives. - Les phénomènes de polyembryonie sont un argument indirect contre la détermination épigamique. - Expériences civerses sur les Végétaux.

Chaprtar XIII. - L'époque de la détermination du sexe

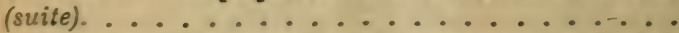

II. - Hypothèse progamique. - Théories de Beard et de Lenhossek. - Actions exercées sur l'ovule en voie de croissance: expériences de Russo. - Les espèces à cufs dimorphes; cas de Dinophilus. 
III. - Hypothèse syngamique. - Cas des Rotrfères (Hydatina senta) et des Abeilles. - Théorie de Dzierzon; discussions récentes. .

La loi de Thury; théorie de la transmission croisee dio sexe. - Expériences de R. Hertwig sur les Grenouilles et de miss $H$. King sur les Crapauds.

Chapitre XIV. - Les chromosomes des gamètes et la détermination du seze...........

Le chromosome accessoire dans la spermatogénèse de divers Insectes. - Dimorphisme nucléaire des spermatides. - Théorie d'Ed. Wilson. - Cas divers. Généralisation. - Otjections et restrictions de fait et de principe. - Valeur qualitative ou quantitative des chromosomes spéciaux. - Leur présence dans des animaux hermaphrodites. - Cas des Pucerons.

Chapitre XV. - Le sexe considéré comme caractère

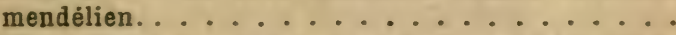

Exposé sommaire des lois de Mendel. - Les deux sexes considérés conme formant un couple de gènes mendéliens. - Expérience de Correns sur la Bryone; interprétation de Correns et de Bateson. - Caractères transmis corrélativement à un sexe (sex-limited inheritance) : expériences sur un Papillon (Abraxas grossulariata). - Mendélisme et polymorphisme sexuel des Papillons.

Conclusions générales relatives au problème de la détermination du sexe.

\section{QUATRIĖUE PARTIE}

\section{LA. PARTHÉNOGÉNĖSE}

Chapttre XVI. - La parthénogẻnèse naturelle. . . 203

Défnition. - La parthénogénèse est un processus secondaire et non primitif.

Ses degrés : rudimentaire ou complète. - Son exten-. sion chez les animaux et les végétaux. - Ses divers modes : parthénogénèse accidentelle, facultatice. normale, cyclique (régulière ou irrégulière), constante. - Disnarition des máles : exemples divers. 
ChapItre XVII. - La parthénogénèse naturelle (suite) - Déterminisme de la réapparition de la bisexualitẻ chez les animaux à parthénogénèse cyclique

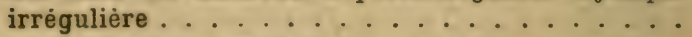

Cas des Rotifères (Hydatine). - Expériences de Maupas (rôle de la température), de Nussbaum (rôle de la nutrition), de Punnett (rôle de l'hérédité), de Shull et de Whitney (rôle de la composition chimique du milieu).

Cas des Crustacés Cladocères. - Travaux de Weismann : cycles héréditaires et actions du milieu.

Cas des Pucerons: râle de la température. - Expériences de Kyber, etc. - Le cycle des Chermes. Travaux de P. Marchal. - Stabilisation progressive des modifications produites par le milieu. - Disparition progressive du sexe mále (spanandrie).

Chapitre XVIII. - La parthénogénèse expérimentale.

Réalisation expérimentale de la parthénogénèse, par des agents physico-chimiques (J. Lœb). - Possibilite générale de l'activation de l'ovule par cette voie. La dualité de la fécondation : amphimixie héréditaire, activation mécanique de l'avule. - La fécondation est ainsi dépouillée d'un aspect vitaliste.

Extension actuelle et catégories principales des faits de parthénogénèse expérimentale. - Vues sommaires sur les théories proposées pour en expliquer le mécanisme : Cytolyse et régulation des oxydations (Lœb); coagulations et liquéfactions des collö̈des ovulaires (Delage); déshydratation usmotique et élimination des déchets (Bataillon).

Chapitre XIX. - La parthénogénèse et la réduction

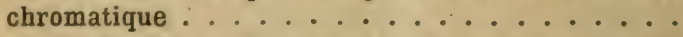

Comment la réduction chromatique dans l'ovule est-elle compensée, en l'absence d'une fécondation? - Le nombre des globules polaires dans les cufs parthénogénétiques. - La réduction chromatique chez le mâle des Abeilles. - Cas de conjugaison du second globule polaire et du' noyau de l'ovule. - Possibilité ciune autorégulation des chromosomes. 


\section{CINQUTĖYE PARTIE}

\section{SEXUALITÉ ET MULTIPLICATION ASEXUÉE}

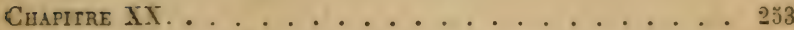

La multiplicátion aseicué : division (schizogénèse), bourgeonnement (blastogénèse). - Dirision des Turbellariés et des Vers. - Stolonisation des Syllidiens; ses rapports avec la reproduction sexuée. - La reproduction des Cestodes et des Trématodes.

Le bourgeonnement chez les animaux coloniaux. Polymorphisme; Dissociation de l'individualité.

La polyembryonie et la reproduction asexuée.

L'existence de la reproduction asexuée est corrélative des conditions qui affaiblissert l'individualité.

\section{SIXIĖYIE PARTIE}

\section{LA SEXUALITÉ CHEZ LES VÉGÉTAUX ET LES ORGANISMES INFÉRIEURS (PROTOZOAIRES ET THALLOPHYTES)}

Chaptrre XXI. - La sexualité chez les Végétaux (non Thallophytes). . . . . . . . . . . .

1. - Archégoniées. - Archégone et anthéridie. - Fougères : plante feuillée et prothalle; sporophyte et gamétophyte (génération diploïde et génération haploïde). - Alternance des générations. - La réduction chromatique a lieu lors de la formation du gamétophyte et non lors de la formation des gamètes.

II. - Phanérogames. - Les gamètes et la fécondation. - Le grain de pollen est un prothalle mâle rudimentaire. - Anthéro:iödes du Cycas et du Gincko. - La transformation du gamète mále est corrélative de ce que la fécondation saccomplit à sec. - Régression du prothalle femelle des Phanérogames. Cas transitionnel des Gynnospermes. - Les Ptéridospermées fossiles. 
Chapitre XXII. - La sexualité chez les Protozoaires. .

Diversité des phénomènes de sexualité cliez les organismes inférieurs.

La sexualité des Infusoires. - Travaux d'E. Maupas: le rajeunissement caryogamique. - Isogamie et anisogamie. - Recherches de Calkins, Enriques, Woodruff. - La sexualité chez les autres Protozoaires; les conditions de sa manifestation. - Les Protozoaires parasites (Coccidies, Hématozoaires, etc.).

L'autogamie chez les Protozoaires.

Chapitre XXIII. - La sexualité chez les Thallophytes.

Peproduction asexuée: spores, zoospores. - Reproduction sexuée par gamètes. - Diversité des gamètes. Cas des Diatomées, Desmidiées, Levures, etc. - Gamètes mobiles (Flagellés). - Isogamie et degrés divers danisogamie. - Characées, Fucus, Volvocinées. Gamétangie des Mucorinées et de divers qutres Champignons.

Sexualité purement physiologique de divers Champignons et Algues. - Mucorinées homothalliques et hétérothalliques.

L'autogamie chez les Thallophytes.

Chapitre XXIV. - Les conditions générales de la sexualité dans les organismes inférieurs. . . . . . 309 Y a-t-il chez eux des globules polaires et une réduction chromatique?

Gènéralité des phénomènes de sexualité chez les Protozoaires et les Thallophytes. - Leur diversité. Il se sont différenciés, de faşons indépendantes, dans les divers groupes.

\section{CONCLUSION}

Chafitre XXY. - Vues générales sur les problèmes

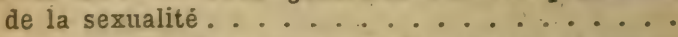

Lans quelle mesure la sexualité est-elle liée à la reproduclion? - Cn dernier mot sur les rapports de l'hermaphrodisme et du gonochorisme. - Conclusions relatives à la détermination du sexe. - La sexualité et l'ensemble de lorganisme. - Le sexe se détermine-t-il dans le soma ou dans le germen?

$$
\text { 4489-4-19. - Paris - Imp. Hemmerlé et } \mathbf{O}^{40} \text {. }
$$




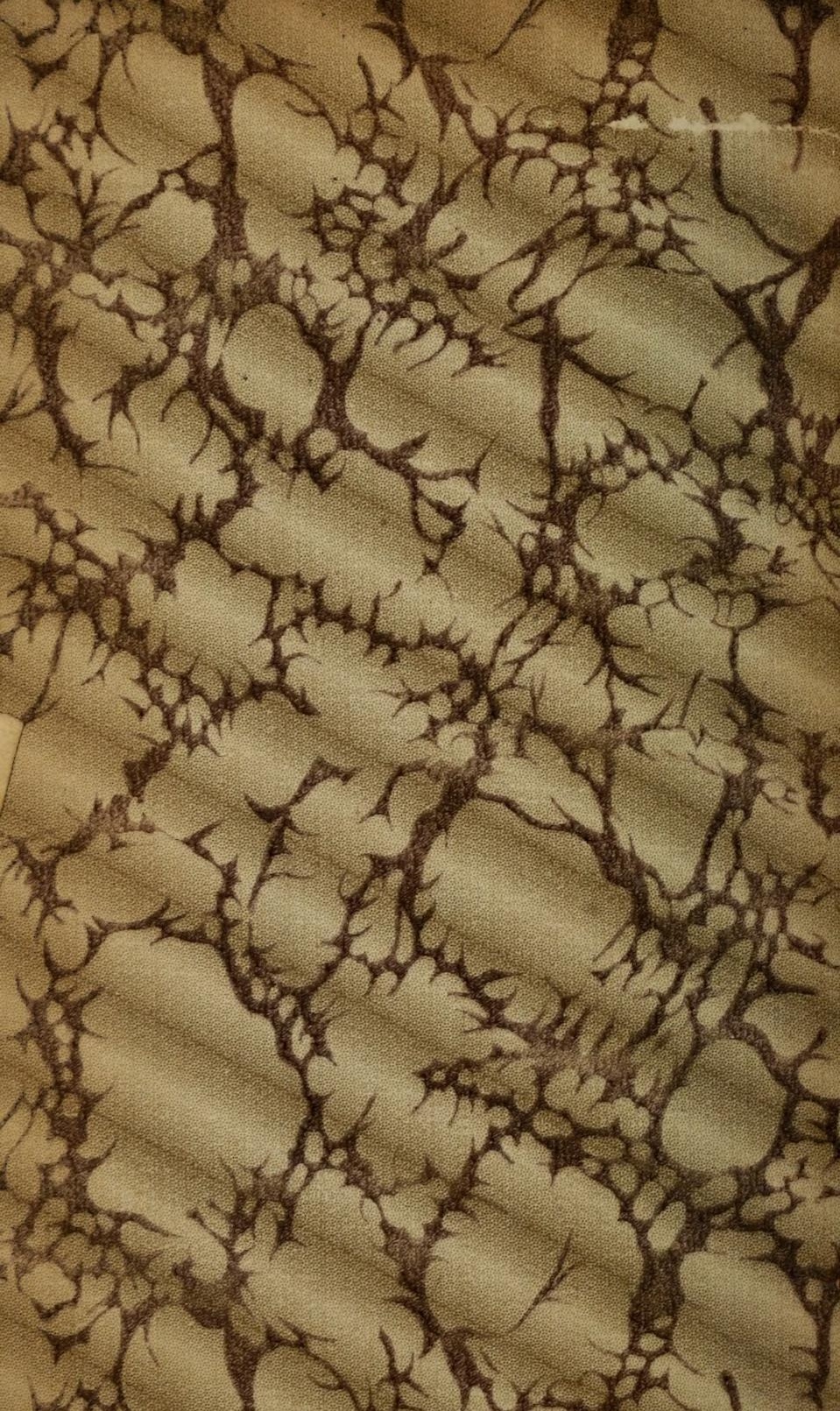


QH Caullery, Maurice Jules Gaston 481 Corneille

C38 Les problèmes de la

1919 sexualité

BioMed

\section{PLEASE DO NOT REMOVE}

CARDS OR SLIPS FROM THIS POCKET

- UNIVERSITY OF TORONTO LIBRARY 
